University of South Florida

DIGITAL COMMONS

Digital Commons @ University of

@ UNIVERSITY OF SOUTH FLORIDA

South Florida

4-7-2014

\title{
A 3-D Pseudo-Rigid-Body Model for Rectangular Cantilever Beams with an Arbitrary Force End-Load
}

Jairo Renato Chimento

University of South Florida, jairo.chimento@gmail.com

Follow this and additional works at: https://digitalcommons.usf.edu/etd

Part of the Mechanical Engineering Commons

\section{Scholar Commons Citation}

Chimento, Jairo Renato, "A 3-D Pseudo-Rigid-Body Model for Rectangular Cantilever Beams with an Arbitrary Force End-Load" (2014). USF Tampa Graduate Theses and Dissertations.

https://digitalcommons.usf.edu/etd/4993

This Dissertation is brought to you for free and open access by the USF Graduate Theses and Dissertations at Digital Commons @ University of South Florida. It has been accepted for inclusion in USF Tampa Graduate Theses and Dissertations by an authorized administrator of Digital Commons @ University of South Florida. For more information, please contact digitalcommons@usf.edu. 
A 3-D Pseudo-Rigid-Body Model for Rectangular Cantilever Beams with an Arbitrary Force End-Load

by

Jairo R. Chimento

A dissertation submitted in partial fulfillment

of the requirements for the degree of

Doctor of Philosophy in Mechanical Engineering

Department of Mechanical Engineering

College of Engineering

University of South Florida

\author{
Major Professor: Craig Lusk, Ph.D. \\ Alex Volinsky, Ph.D. \\ Nathan Crane, Ph.D. \\ Tapas Das, Ph.D. \\ Gloria Wiens, Ph.D.
}

Date of Approval:

April 7, 2014

Keywords: Compliant Mechanisms, Kinematics, Non-Dimensional, Large Deflections, Spatial Deflections

Copyright (C 2014, Jairo R. Chimento 


\section{DEDICATION}

To my wife, Yisset. 


\section{ACKNOWLEDGMENTS}

The author wishes to acknowledge all the efforts and support of the people who contributed to this dissertation. My gratitude is devoted to Dr. Craig Lusk whose patience, guidance and encouragement allowed me to materialize this dream.

The time and effort devoted from the committee members is deeply appreciated.

My thanks to the compliant mechanisms research group that helped through this research suggesting solutions to occasional problems. Special thanks to Ahmad Alqasimi for his contribution on CAD and FEA software support.

The authors of this work gratefully acknowledge the support of the National Science Foundation, Grant \#CMMI-1000138. 


\section{TABLE OF CONTENTS}

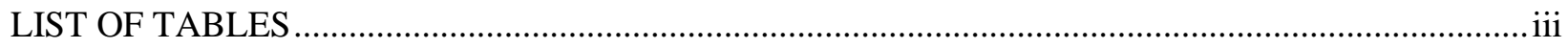

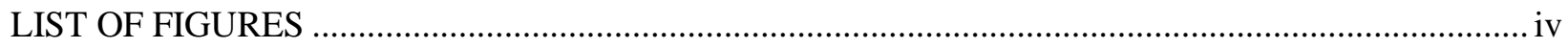

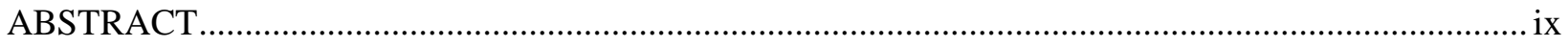

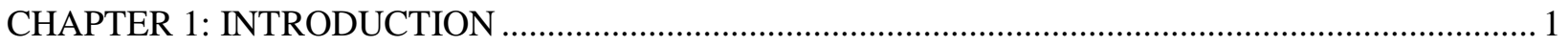

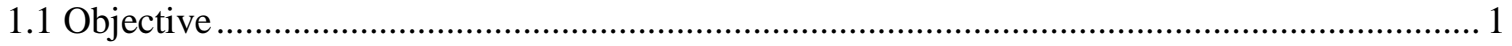

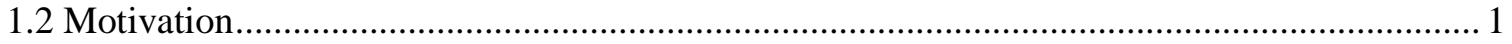

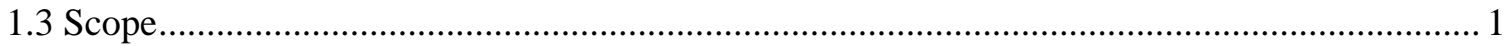

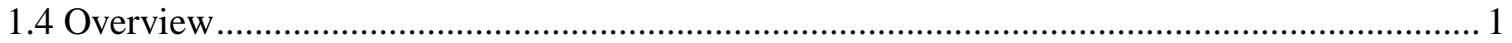

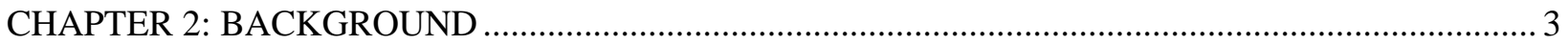

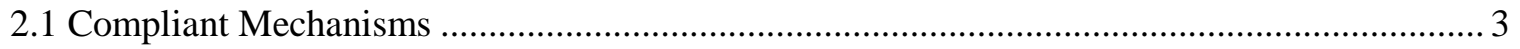

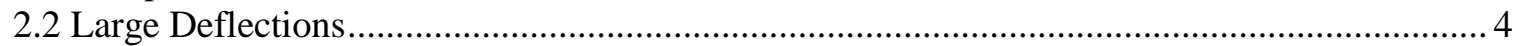

2.3 Planar Pseudo-Rigid-Body Concept ........................................................................................... 5

2.4 Planar Cantilever Beam with a Force at the Free End (Fixed-Pinned) ..................................... 6

2.5 Follower and Non-Follower Forces ........................................................................................ 8

2.6 PRBMs for Planar Cantilever Beams with a Force at the Free End (Fixed-Pinned) ................ 10

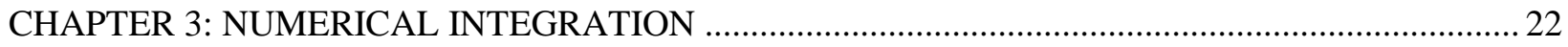

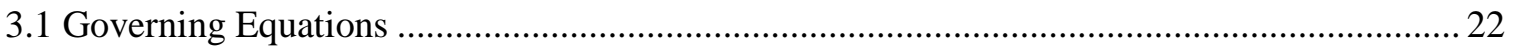

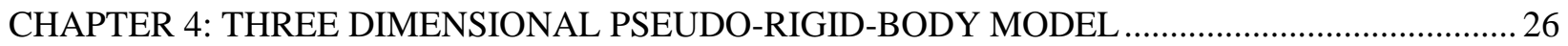

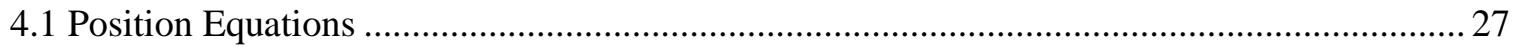

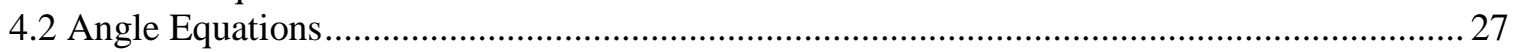

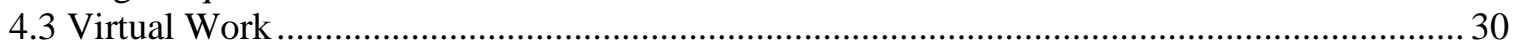

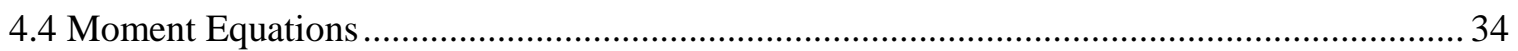

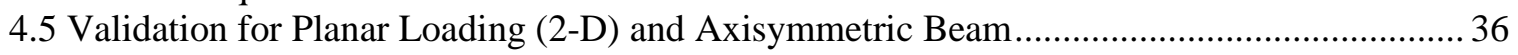

4.6 Validation Using Finite Element Analysis...................................................................... 40

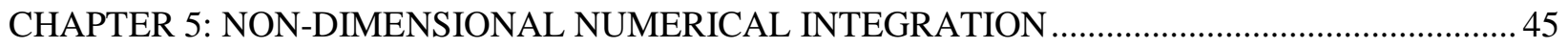

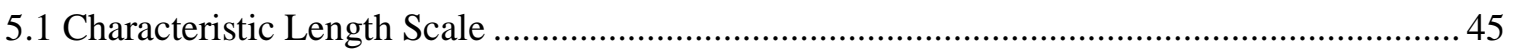

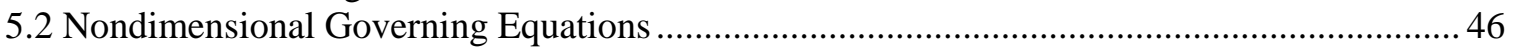

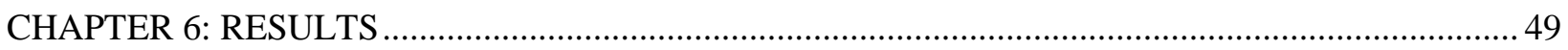

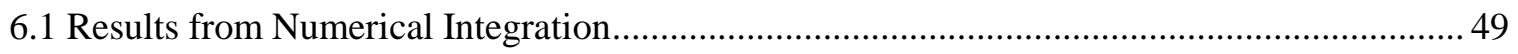

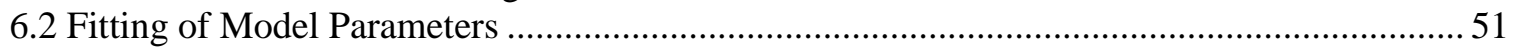

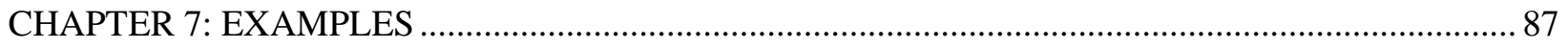

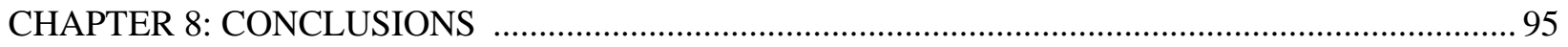




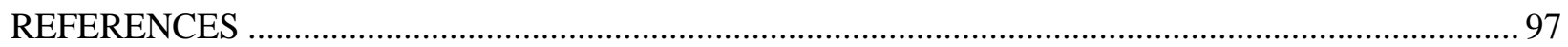

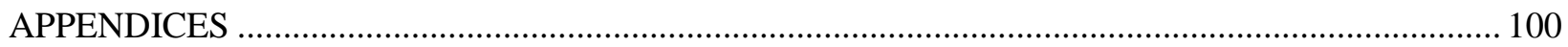

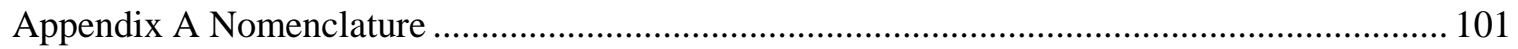

Appendix B Regressions and Statistical Values for PRBM Parameters.................................... 102

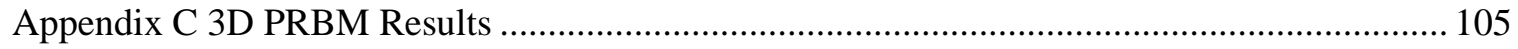

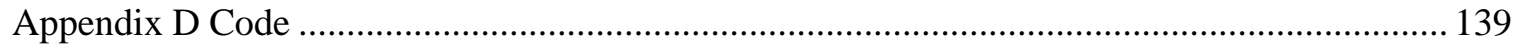




\section{LIST OF TABLES}

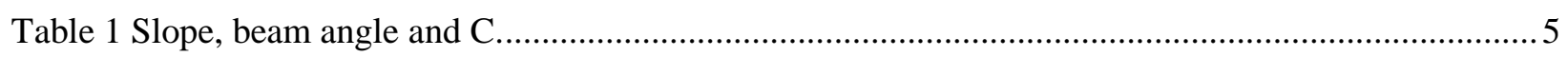

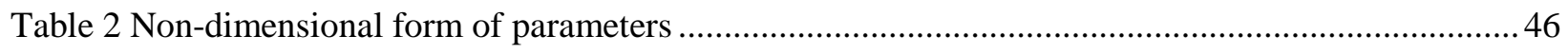

Table 3 Typical results from numerical integration........................................................................ 48

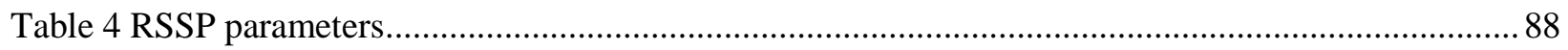

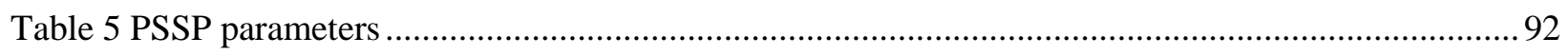

Table 6 Results from compliant spatial double slider ........................................................................ 94

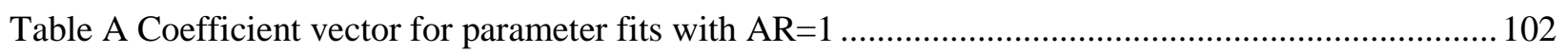

Table B Coefficient vector for parameter fits with AR=0.8 ......................................................... 102

Table C Coefficient vector for parameter fits with AR=0.5 ........................................................... 103

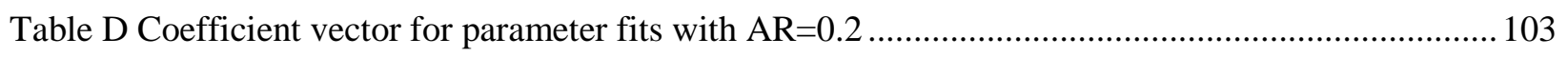

Table E Average and standard deviation of PRBM constants for AR=1 ............................................ 103

Table F Average and standard deviation of PRBM constants for AR=0.8 ...................................... 104

Table G Average and standard deviation of PRBM constants for AR=0.5 ….................................... 104

Table H Average and standard deviation of PRBM constants for AR=0.2 …................................... 104 


\section{LIST OF FIGURES}

Figure 1 Cantilevered segment with forces at the free end ........................................................... 7

Figure 2 Howell's PRBM of a cantilever beam (fixed-pinned)....................................................... 7

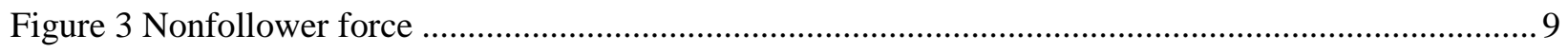

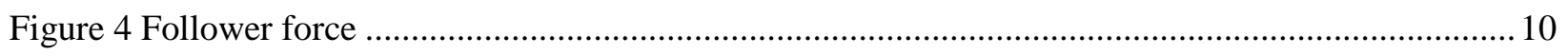

Figure 5 Pseudo-rigid-body models with multiple torsional springs ................................................. 13

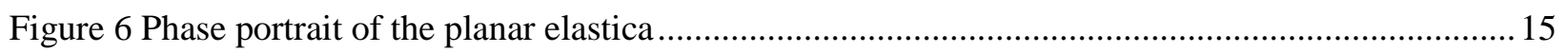

Figure 7 Phase-portrait showing non-dimensional curvature versus slope of the elastica........................ 17

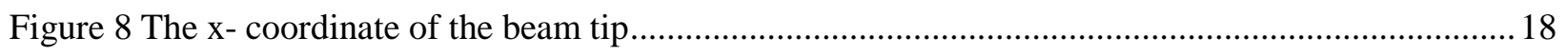

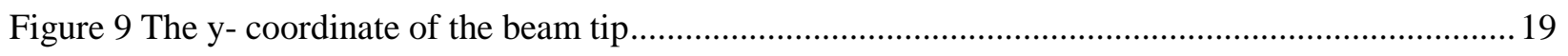

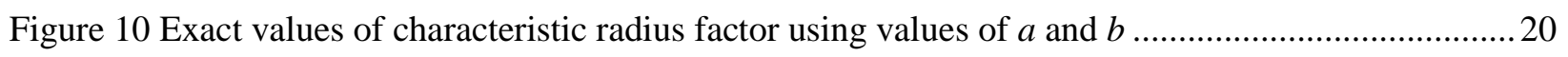

Figure 11 Relative error incurred from using $\gamma$ value of 0.85 in PRBM equations ..................................2 21

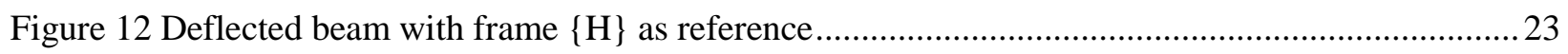

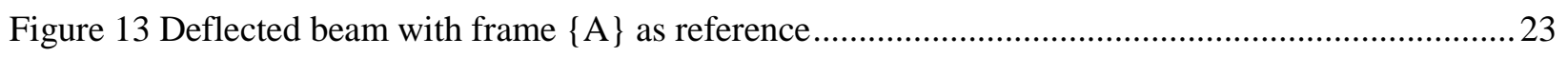

Figure 14 Force direction described with spherical coordinates...........................................................2 24

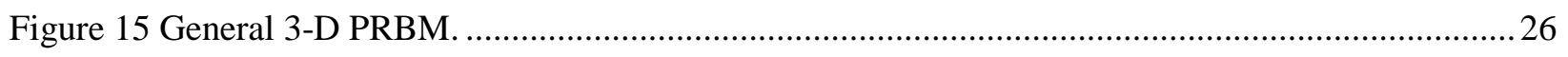

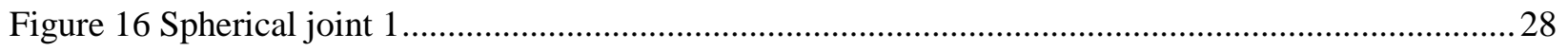

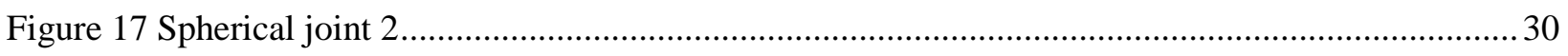

Figure 18 Spherical joint 1 with twisting and orientation angles........................................................... 30

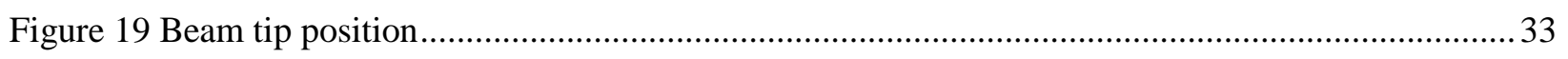

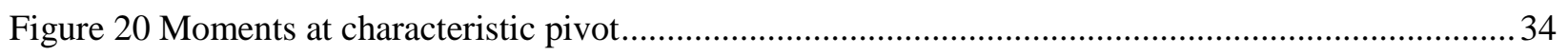

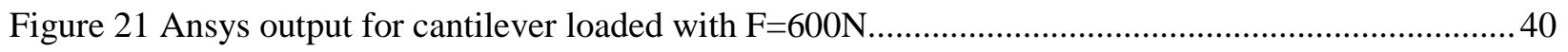


Figure 22 Deformed and undeformed beam loaded with 600N........................................................ 41

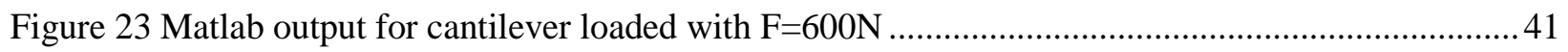

Figure 24 Ansys output for cantilever loaded with F= 750N............................................................ 42

Figure 25 Deformed and undeformed beam loaded with 750N.......................................................... 43

Figure 26 Matlab output for cantilever loaded with $\mathrm{F}=750 \mathrm{~N}$............................................................ 43

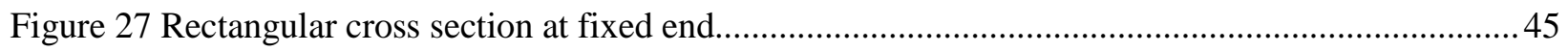

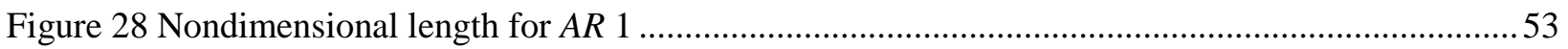

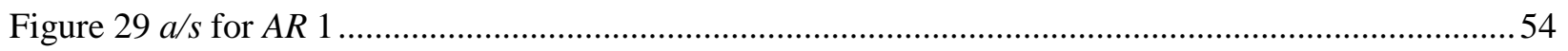

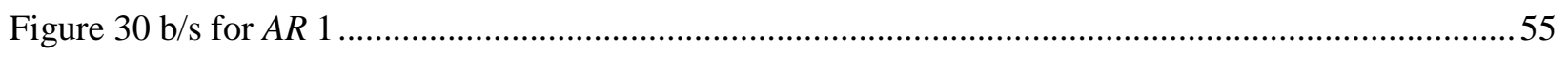

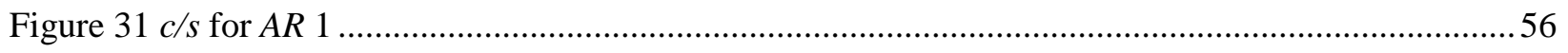

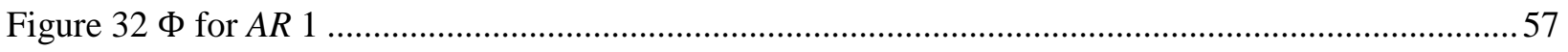

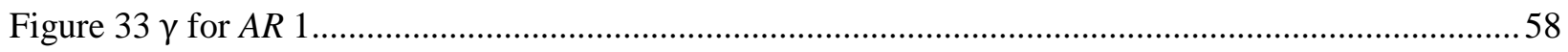

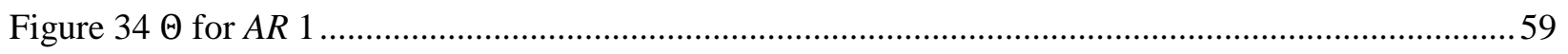

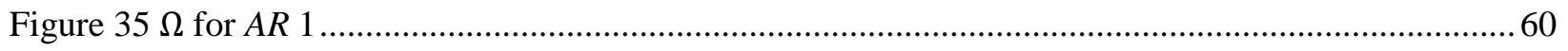

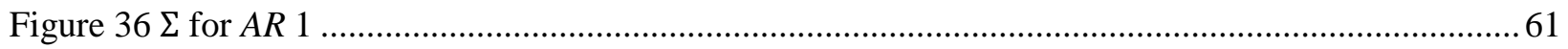

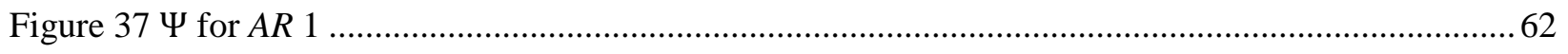

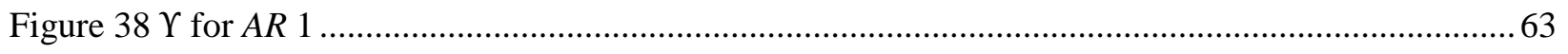

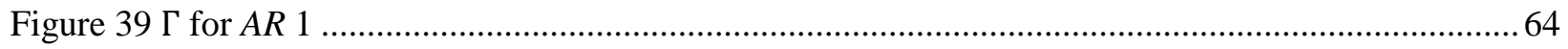

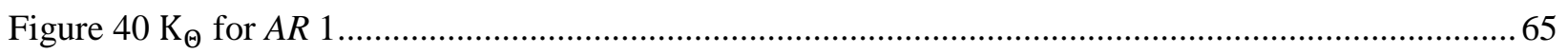

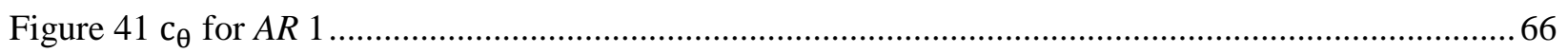

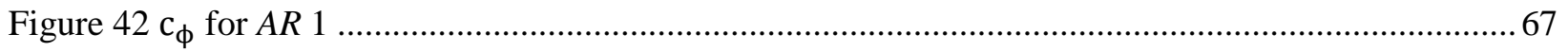

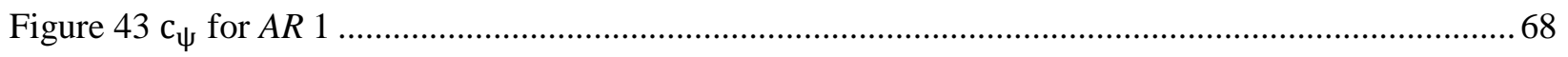

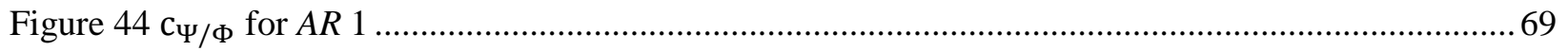

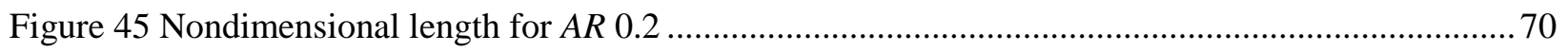




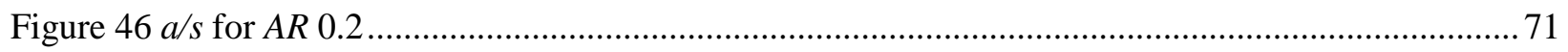

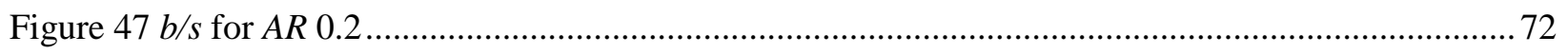

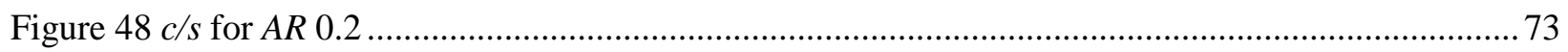

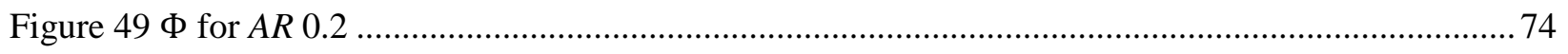

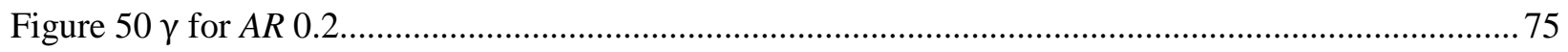

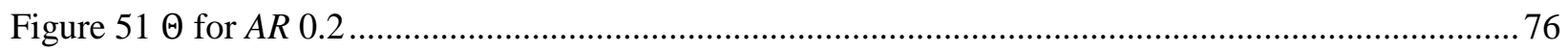

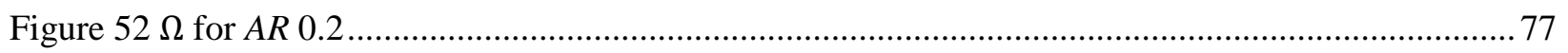

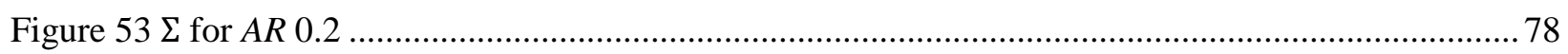

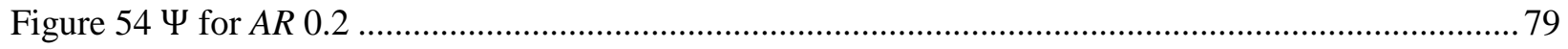

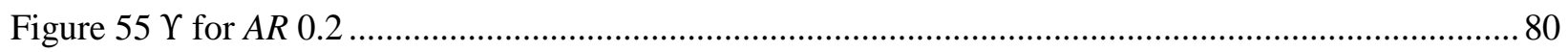

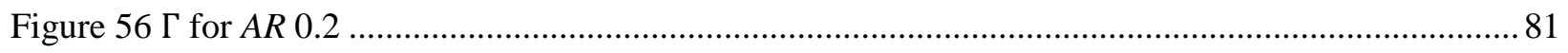

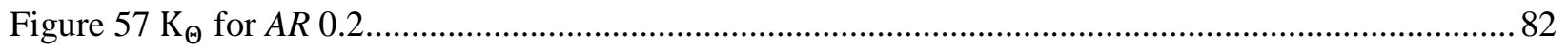

Figure $58 c_{\theta}$ for $A R$ 0.2

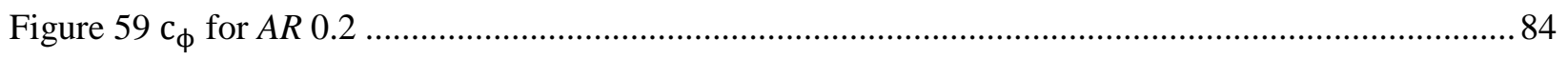

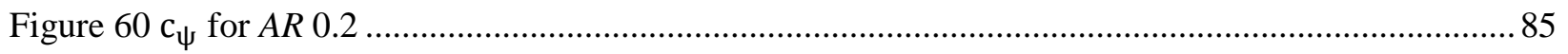

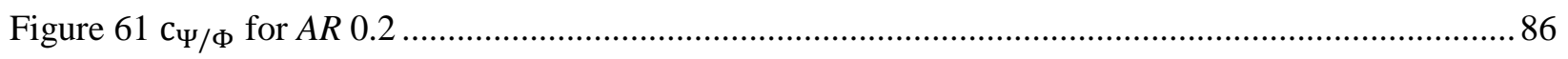

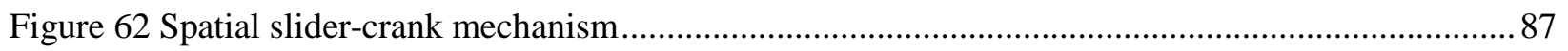

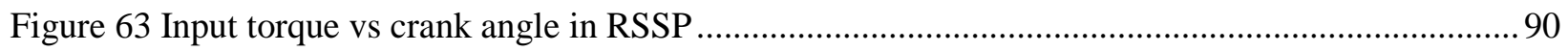

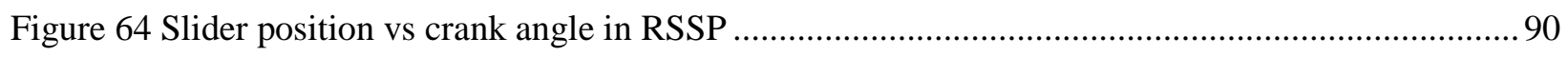

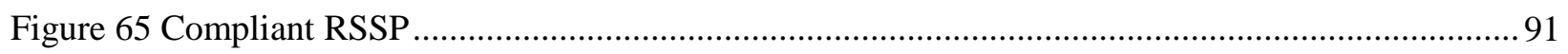

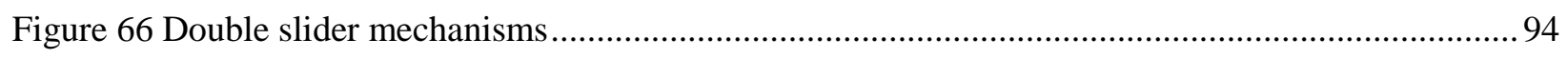

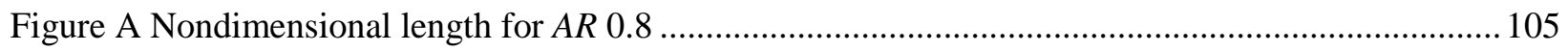

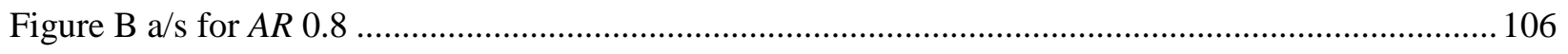

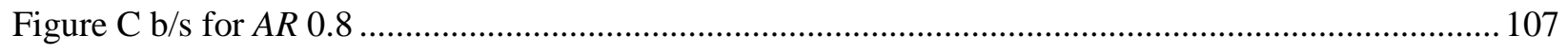




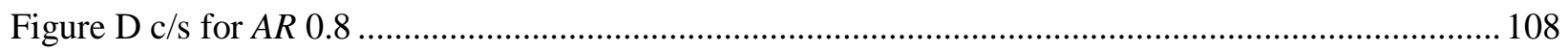

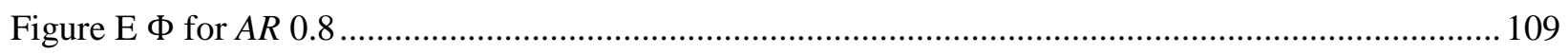

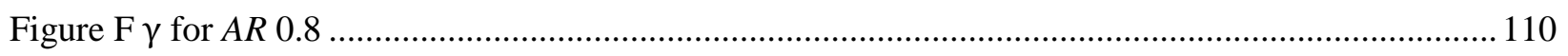

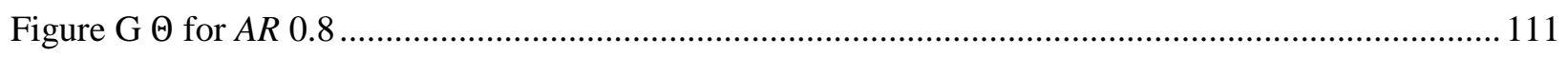

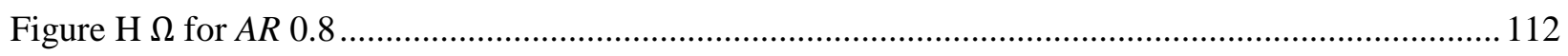

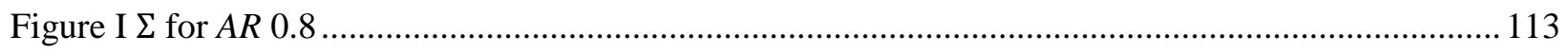

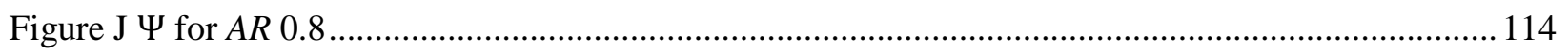

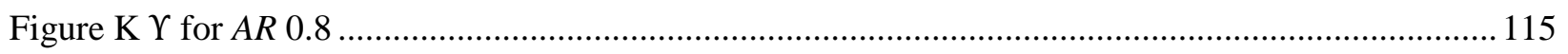

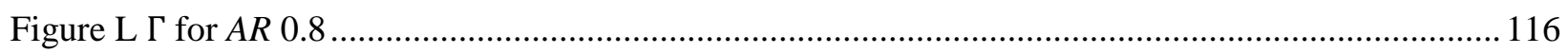

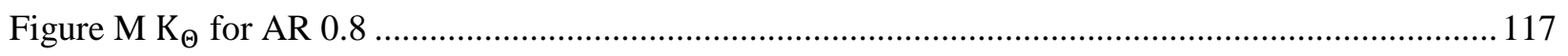

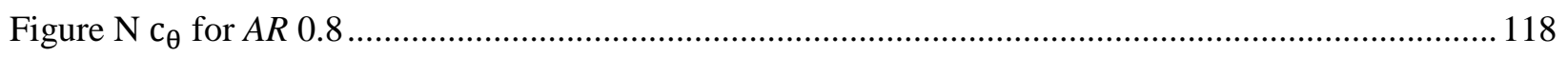

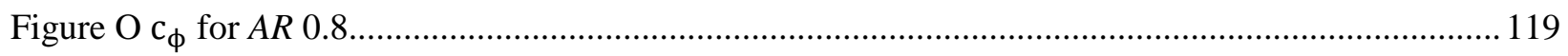

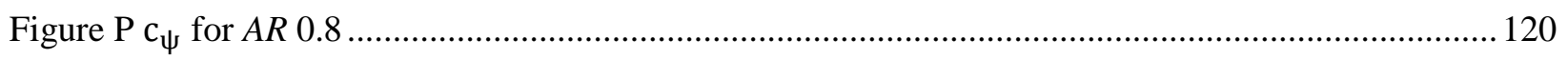

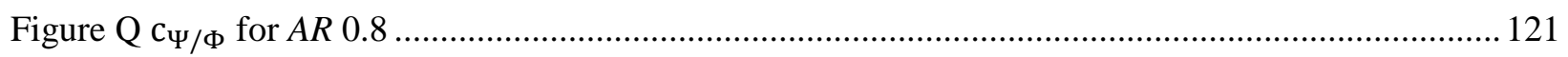

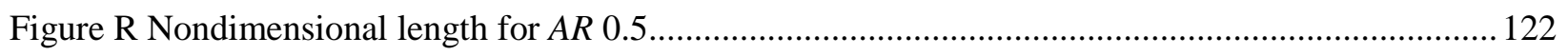

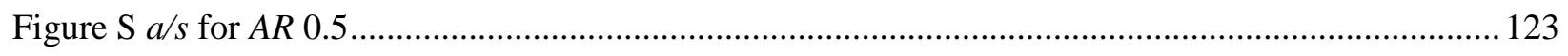

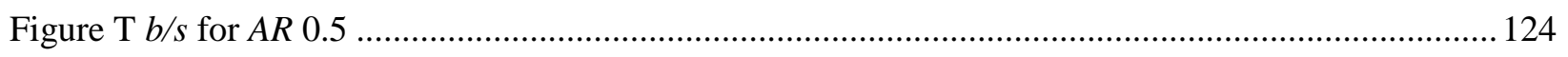

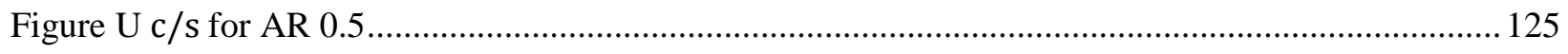

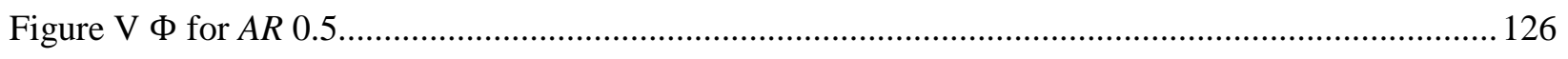

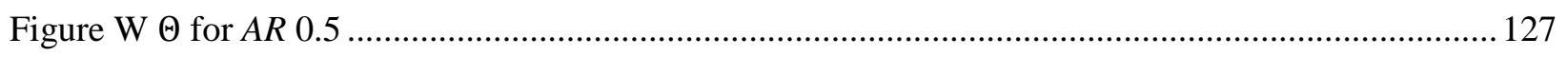

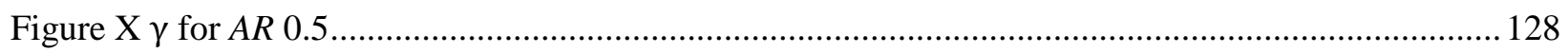

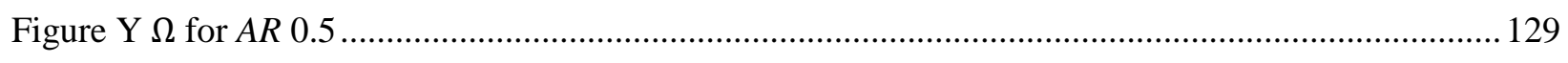

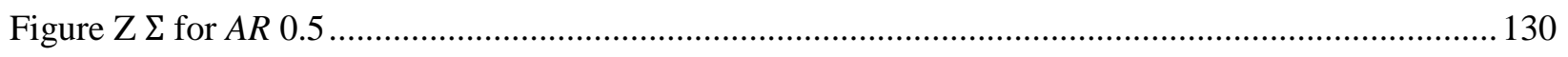

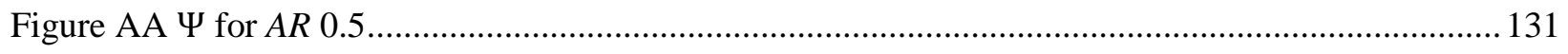




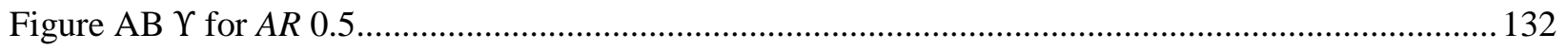

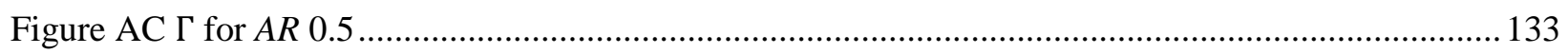

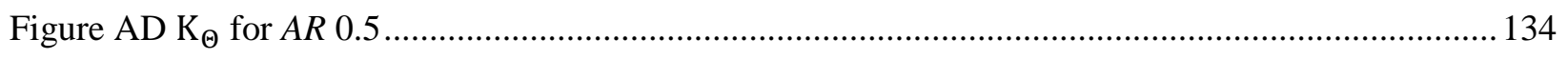

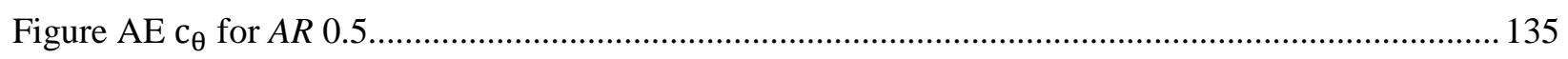

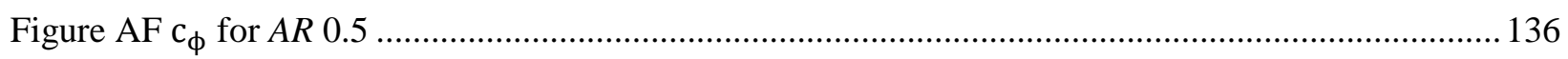

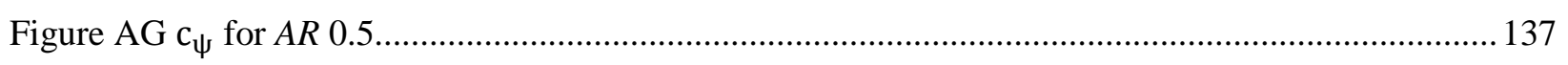

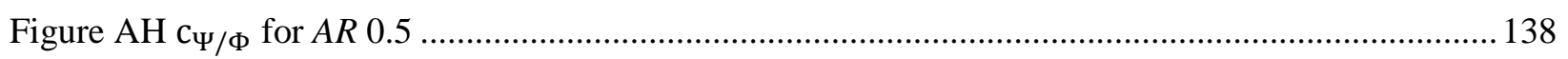




\begin{abstract}
This dissertation introduces a novel three-dimensional pseudo-rigid-body model (3-D PRBM) for straight cantilever beams with rectangular cross sections. The model is capable of capturing the behavior of the neutral axis of a beam loaded with an arbitrary force end-load. Numerical integration of a system of differential equations yields approximate displacement and orientation of the beam's neutral axis at the free end, and curvatures of the neutral axis at the fixed end. This data was used to develop the 3-D PRBM which consists of two torsional springs connecting two rigid links for a total of 2 degrees of freedom (DOF). The 3-D PRBM parameters that are comparable with existing 2-D model parameters are characteristic radius factor (mean: $\gamma=0.8322$ ), bending stiffness coefficient (mean: $K_{\Theta}=2.5167$ ) and parametric angle coefficient (mean: $c_{\Theta}=1.2501$ ). New parameters are introduced in the model in order to capture the spatial behavior of the deflected beam, including two parametric angle coefficients (means: $c_{\Psi}$ $\left.=1.0714 ; c_{\Phi}=1.0087\right)$. The model is verified in a few locations using ANSYS $^{\mathrm{TM}}$ and its use in the design of compliant mechanisms is illustrated through spatial compliant versions of crank slider and double slider mechanisms.
\end{abstract}




\section{CHAPTER 1: INTRODUCTION}

\subsection{Objective}

The objective of this dissertation is to present the first three-dimensional pseudo-rigid-body model (3-D PRBM) for straight cantilever beams with rectangular cross section. The model is capable of capturing the behavior of the neutral axis of a beam loaded with an arbitrary force end-load.

\subsection{Motivation}

The motivation for this work is to simplify the design of spatial compliant mechanisms, and to provide a means of checking whether planar compliant mechanisms will buckle out-of-plane. Previous PRBM have proved to be effective design tools for planar compliant mechanisms and have resulted in a large number of useful/interesting mechanisms. It is anticipated that the model developed in this dissertation will be useful in mechanisms design.

\subsection{Scope}

The scope of this work is to describe the method used to develop the 3-D PRBM and introduce all its parameters. Additionally, its use is illustrated in spatial compliant versions of crank slider and double slider mechanisms.

\subsection{Overview}

Chapter 2 introduces background information on planar compliant mechanisms and PRBM’s. The governing equations that capture the behavior of the cantilever beams are presented in Chapter 3. The 3-D PRBM and its parameters are described in Chapter 4. The PRBM equations are derived using virtual work. Chapter 5 presents the governing equations in non-dimensional form. Typical parameters values are 
summarized for different aspect ratios in Chapter 6. These results will assist engineers in making quick approximations of the deflections of three-dimensional cantilever beams. Two examples are presented in Chapter 7 to demonstrate the usefulness of the model. The conclusions and contributions of this dissertation are explained in Chapter 8. 


\section{CHAPTER 2: BACKGROUND}

\subsection{Compliant Mechanisms}

A compliant mechanism transfers motion, force or energy as a result of the elastic deflection of flexible members. Compliant mechanisms gain at least some of their mobility from the deflection of flexible members rather than from movable joints only [1]. Compliant mechanisms can be categorized by the presence of kinematic pairs as either fully compliant or partially compliant mechanisms. Fully compliant mechanisms do not have kinematic pairs. In fact, they transfer the energy from input to output only through the deflection of flexible members. On the other hand, partially compliant mechanisms may have one or more joints, including pins and sliders.

Compliant mechanisms offer advantages to the designers in two main categories: reduced cost (part-count reduction, reduced assembly time, simplified manufacturing processes) and increased performance (increased precision, increased reliability, reduced wear, reduced weight, reduced maintenance) [1].

Compliant mechanisms advantages offer solutions to Micro-Electro-Mechanical Systems (MEMS) fabrication and assembly challenges. Numerous difficulties arise from MEMS design and part assembly of mechanical and electrical components on the micrometer scale. First, the performance of hinges and pin joints in the micro-scale do not exactly resemble their counterparts at the macro-scale. Second, due to the planar nature of the MEMS fabrication, it can be challenging to produce spatial or three dimensional motion mechanisms.

Compliant mechanisms also present several challenges and disadvantages in some applications. One limitation of compliant mechanisms is the storage of energy in the flexible segments [2]. Inside the 
elastic range of the material, flexible segments build up potential energy in the form of strain energy when they experience deflection. For some applications, this behavior is undesirable because all the energy provided at the input is not transferred to the output. Another disadvantage in compliant mechanisms is the fact that compliant links that remain under stress for long periods of time or at high temperatures may experience stress relaxation or creep [1]. Finally, the largest challenge is to predict with sufficient accuracy the behavior of compliant mechanisms.

\subsection{Large Deflections}

Geometric nonlinearities are introduced in the analysis of compliant mechanisms when compliant flexures experience large deflections. "The major difference between large- and small-deflection analysis lies in the assumptions made to solve the Bernoulli-Euler equation’’ [1], The Bernoulli Euler equation is:

$$
M=E I \frac{d \theta}{d s}
$$

where $M$ is the moment, $\frac{d \theta}{d s}$ the rate of change in angular deflection along the beam (curvature), $E$ the Young's modulus of the material, and $I$ the beam moment of inertia. The curvature can be expressed as

$$
\frac{d \theta}{d s}=\frac{\frac{d^{2} y}{d x^{2}}}{\left[1+\left(\frac{d y}{d x}\right)^{2}\right]^{3 / 2}}
$$

where $y$ is the transverse deflection and $x$ is the coordinate along the undeflected beam axis. Equation (2) can be written as

$$
\frac{d \theta}{d s}=C \frac{d^{2} y}{d x^{2}}
$$


"For small deflections, it is assumed that $C=1$. As the deflection increases, $C$ changes, and its deviation from unity represents the factor by which the small deflection theory assumption is inaccurate.”

Table 1 Slope, beam angle and C. Adapted from [1]

\begin{tabular}{ccc}
\hline$\frac{\boldsymbol{d y}}{\boldsymbol{d x}}$ & $\boldsymbol{\theta}$ & $\boldsymbol{C}$ \\
\hline 0.01 & 0.6 & 0.9999 \\
0.05 & 2.9 & 0.9963 \\
0.10 & 5.7 & 0.9852 \\
0.25 & 14.0 & 0.9131 \\
0.50 & 26.6 & 0.7155 \\
1.00 & 45.0 & 0.3536 \\
2.00 & 63.4 & 0.0894 \\
\hline
\end{tabular}

Compliant mechanisms undergo large non-linear deflections for which standard small-deflection theory is inadequate. Thus, the mathematical approach for the analysis of planar compliant mechanisms is based on elliptical integrals or Finite Element Analysis (FEA). FEA can predict the behavior of the flexible segments when they are subjected to force or displacement loadings. However, these methods can be time consuming and require large computational power for the early design stage of compliant mechanisms.

\subsection{Planar Pseudo-Rigid-Body Concept}

Alternatively, a popular, easier and more intuitive technique called Pseudo-Rigid-Body Model (PRBM) is used to analyze compliant links. PRBM is a parametric approximation in which the flexural link is modeled as two rigid links joined by a torsional spring. The stiffness coefficient of the spring is determined by the material properties and geometry of the compliant segment. The location of the joint is determined by the characteristic radius factor $\gamma$. The torsional spring imitates the stiffness of the flexible 
member in compliant mechanisms [3]. Currently there exist numerous PRBMs for different flexural segments including small-length flexural pivots (living hinges), cantilever beams with force at free end, fixed guided beams, end-moment loaded cantilevers, initially curved cantilevers and pinned-pinned segments proposed by Midha, Howell, Saxena and Dado [4-9]. These PRBMs have helped the analysis

and synthesis of compliant mechanisms in different applications including pantographs [10], centrifugal clutches [11-12], MEMS devices [13-16]. It is important to mention that these PRBMs are planar, and are constrained to the plane of deflection of the compliant segments.

The focus of attention of this work is a cantilever beam of rectangular cross section with an arbitrary force at the free end which can produce three dimensional motion. This compliant segment requires a novel 3-D PRBM to describe the path of the deflected beam while maintaining the forcedeflection characteristics of the cantilever beam. Since this dissertation presents the first 3-D PRBM for cantilever beams with rectangular cross section, there is no theoretical background for 3-D PRBM. The following section will review the most relevant knowledge and concepts associated with planar cantilever beams with force at the free end. These planar concepts will be explained in detail to ensure the understanding of the reader when this dissertation builds the bridge from 2-D to 3-D motion.

\subsection{Planar Cantilever Beam with a Force at the Free End (Fixed-Pinned)}

A flexible cantilever beam with constant cross section and linear material properties is shown in Figure 1. Its' PRBM equivalent is shown in Figure 2 which "shows a pseudo-rigid-body model of a largedeflection beam for which is assumed that the nearly circular path can be accurately modeled by two rigid links that are joined at a pivot along the beam. A torsional spring at the pivot represents the beam's resistance to deflection. The location of this pseudo-rigid-body characteristic pivot is measured from the beam's end as a fraction of the beam's length, where the fractional distance is $\gamma 1$ and $\gamma$ is the characteristic radius factor. The product $\gamma 1$, the characteristic radius, is the radius of the circular deflection path traversed by the end of the pseudo-rigid-body link. It is also the length of the pseudo-rigid-body link. The 
pseudo-rigid-body angle, $\Theta$, is the angle between the pseudo-rigid-body link and its undeflected position.” In addition, the horizontal (x-coordinate) and vertical (y-coordinate) coordinates of the end of the deflected beam are represented by a and b, respectively.

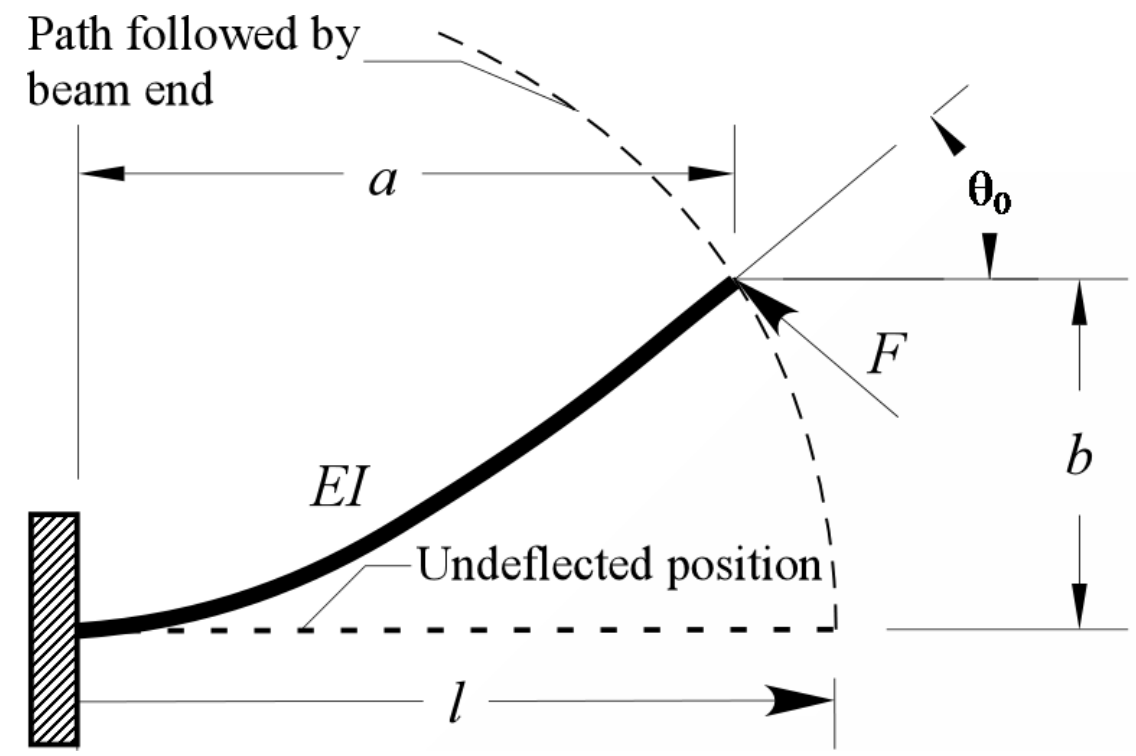

Figure 1 Cantilevered segment with forces at the free end. Adapted from [1]

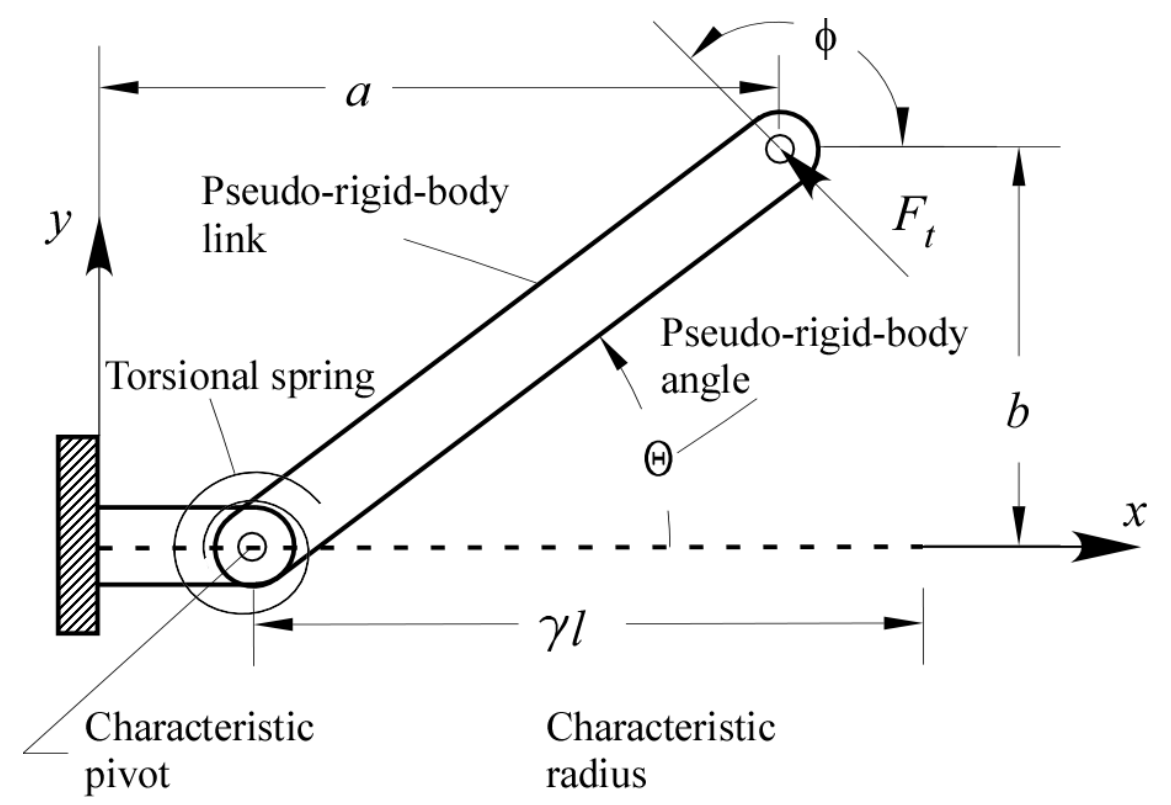

Figure 2 Howell's PRBM of a cantilever beam (fixed-pinned). Adapted from [1] 
The kinematics of the pseudo-rigid-body model is described by equations (4)-(7).

$$
\begin{gathered}
\frac{a}{l}=1-\gamma(1-\cos \Theta) \\
\frac{b}{l}=\gamma \sin \Theta \\
\Theta=\tan ^{-1}\left(\frac{b}{a-l(1-\gamma)}\right) \\
\gamma=\frac{\left(\frac{b}{l}\right)^{2}+\left(1-\frac{a}{l}\right)^{2}}{2\left(1-\frac{a}{l}\right)}
\end{gathered}
$$

The relationship between the angular deflection of the beam's end, $\theta_{0}$, and the pseudo-rigid-body angle, $\Theta$, is nearly linear as expressed in equation (8)

$$
\theta_{0}=c_{\theta} \Theta
$$

where $c_{\theta}$ is a constant called parametric angle coefficient.

\subsection{Follower and Non-Follower Forces}

A key concept that needs to be emphasized before the explanation of the calculation of Howell's PRBM parameters, is the difference between follower and nonfollower forces. The angles, $\delta$ and $\theta$ which describe force and free end orientations are defined using equations (9)-(12). Subscripts o, and l represent the free end, and the fixed end respectively.

$$
\begin{gathered}
x_{o} * \bar{F}=e^{i \delta_{o}} \\
x_{l} * \bar{F}=e^{i \delta_{l}} \\
x_{l} * \overline{x_{o}}=e^{i \theta_{o l}}
\end{gathered}
$$




$$
\delta_{l}=\delta_{o}+\theta_{o l}
$$

where $\overline{x_{o}}$ and $\bar{F}$ are complex conjugates of the positive $\mathrm{x}$-axis of the free end and force direction respectively. In equations (9)-(11), the first term is the tip of the vector, and the second term is the tail of the vector measuring the angle.

In nonfollower forces, the angle of inclination of the force with respect to the positive x-axis of the fixed end, $\delta_{l}$, remains constant during the beam deflection. However, the angle of inclination of the force with respect to the deformed neutral axis of the beam tip, $\delta_{o}$, and the bending angle, $\theta_{o l}$, change throughout the deflection path. In other words, the force components are constant in the fixed end coordinate system. Figure 3 illustrates the concept, where $\delta_{l}=\delta_{o}+\theta_{o l}$ is constant.

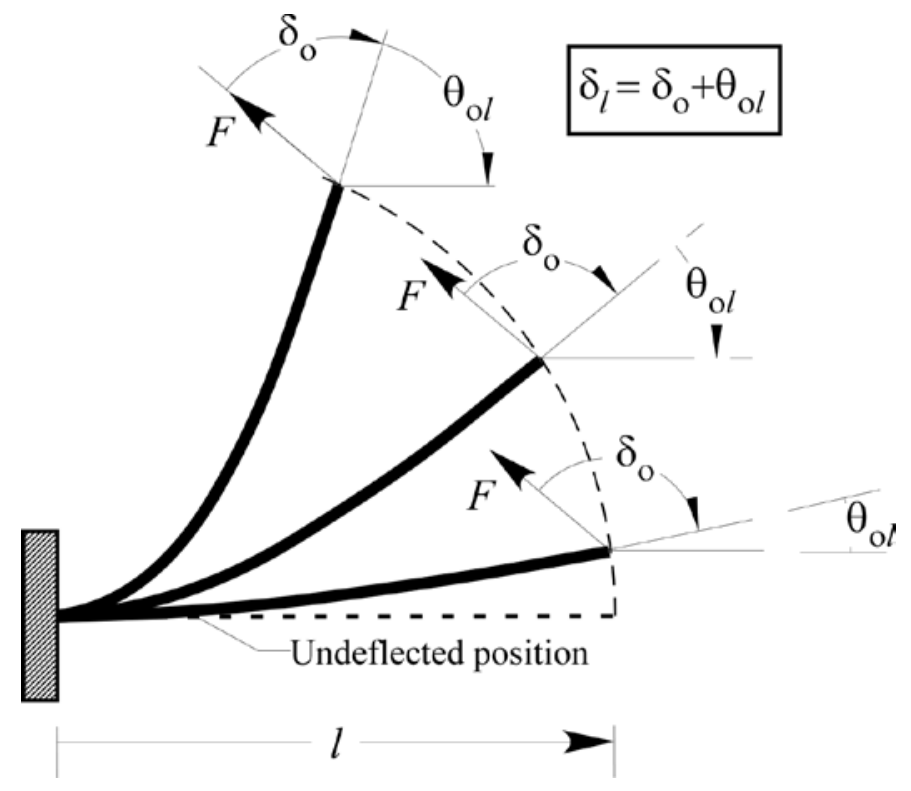

Figure 3 Nonfollower force

On the other hand, in follower forces the angle of inclination of the force with respect to the positive x-axis of the fixed end, $\delta_{l}$, changes during the beam deflection. However, the angle of inclination of the force with respect to the deformed neutral axis of the beam, $\delta_{o}$, remains constant throughout the 
deflection. In other words, the force components are constant in the free end coordinate system. The concept is illustrated in Figure 4.

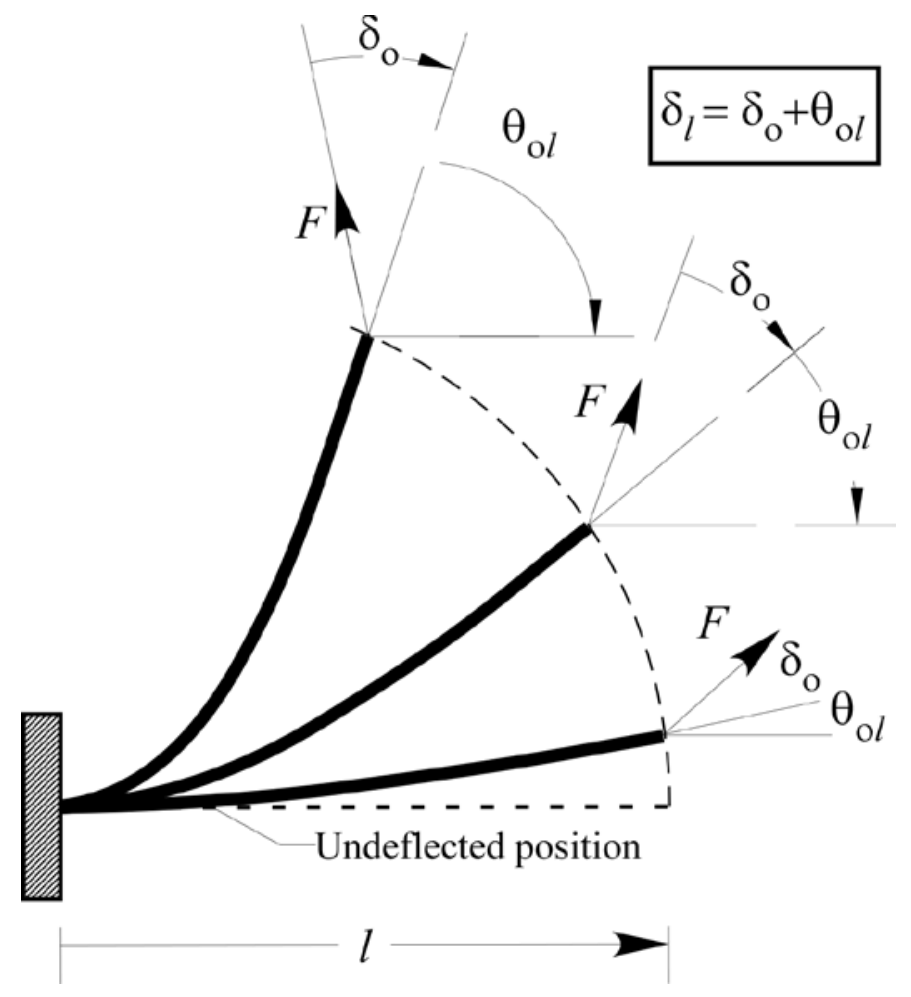

Figure 4 Follower force

\subsection{PRBMs for Planar Cantilever Beams with a Force at the Free End (Fixed-Pinned)}

Howell uses optimization routine to find the values of the characteristic radius factor for planar loading at different nonfollower force angles, $\phi$, which is equal to $\delta$ in the opposite direction. The optimization problem was stated as follows, "Find the value of the characteristic radius factor, $\gamma$, which maximizes the pseudo-rigid-body angle, $\Theta$, where $\Theta=\tan ^{-1}\left(\frac{b}{a-l(1-\gamma)}\right)$ which is subject to the parametric constraint:

$$
g(\Theta)=\frac{\text { error }}{\delta_{e}} \leq\left(\frac{\text { error }}{\delta_{e}}\right)_{\max } \text { for } 0<\Theta<\Theta_{\max }
$$




$$
\frac{\text { error }}{\delta_{e}}=\frac{\sqrt{\left\{\left(\frac{a}{l}\right)-[1-\gamma(1-\cos \Theta)]\right\}^{2}+\left[\left(\frac{b}{l}\right)-\gamma \sin \Theta\right]^{2}}}{\sqrt{\left(1-\frac{a}{l}\right)^{2}+\left(\frac{b}{l}\right)^{2}}}
$$

where $\frac{\text { error }}{\delta_{e}}$ is the relative deflection error, and a and $\mathrm{b}$ the respective horizontal and vertical coordinates of the deflected beam end, which are calculated using elliptic integral approach”.

Howell [4] optimized the characteristic radius factor such that the maximum percent error in deflection was $0.5 \%$ and allowed the maximum range for the pseudo-rigid-body angle. The optimal value found for $\gamma$ is 0.8517 which yields a maximum $\Theta$ of $64.3^{\circ}$ for a nonfollower vertical force.

The bending stiffness of the compliant beam is modeled using a torsional spring with constant spring-rate, $K$, placed at the characteristic pivot as illustrated in Figure 2 . The value of the spring constant is determined using geometric and material properties of the beam, and the non-dimensional stiffness coefficient, $K_{\Theta}$, using equation (16). The stiffness coefficient can be calculated using a non-dimensional expression for the force orthogonal to the pseudo-rigid-link, $F_{t}$, as follows

$$
K_{\Theta}=\frac{F_{t} l^{2}}{E I \Theta}
$$

However, Howell approximated the value of $K_{\Theta}$ to 2.65 for the range of $63^{\circ}<\phi<135^{\circ}$. The equation for the torsion spring constant is:

$$
K=\gamma K_{\Theta} \frac{E I}{l}
$$

For the PRBM shown in Figure 2, the torque, $T$, at the pin joint is proportional to the torsional spring constant, $K$, times the PRBM angle, $\Theta$ : 


$$
T=K \Theta
$$

This torque can also be calculated as the product of the tangential force, $F_{t}$, and the length of the pseudo-rigid link

$$
T=F_{t} \gamma l
$$

Combining equations (16), (17) and (18) an expression for the force required to deflect the compliant member up to $\Theta$.

$$
F_{t}=\frac{E I K_{\Theta} \Theta}{l^{2}}
$$

In addition to Howell's planar PRBM for cantilever beam using one torsional spring (1R), other researchers have developed models with increased numbers of torsional springs, (2R-3R) to increase the range of accuracy of the model. Figure 5 illustrate two examples of PRBMs with multiple torsional springs.

Yue-Qing [17] proposed a PRBM with three rigid links joined by two torsional springs for the cantilever beam with force at the free end. Similarly to Howell's approach, an optimization routine is carried to obtain values for $\gamma$ maximizing $\Theta$ while maintaining the error of the angle deflection below $1 \%$.

The addition of the second pivot and torsional spring increased the maximum PRBM angle, $\Theta$, from $64.3^{\circ}$ (Howell) up to $89.9^{\circ}$ for the cantilever loaded with a vertical force, $\phi=90^{\circ}$.Furthermore, Su [18] proposed a PRBM with four rigid links joined by three pins and three torsional springs for the cantilever beam with combined end force and moment. A specific search routine finds the optimal set of characteristic radius factors and spring stiffness for the different torsional springs. 

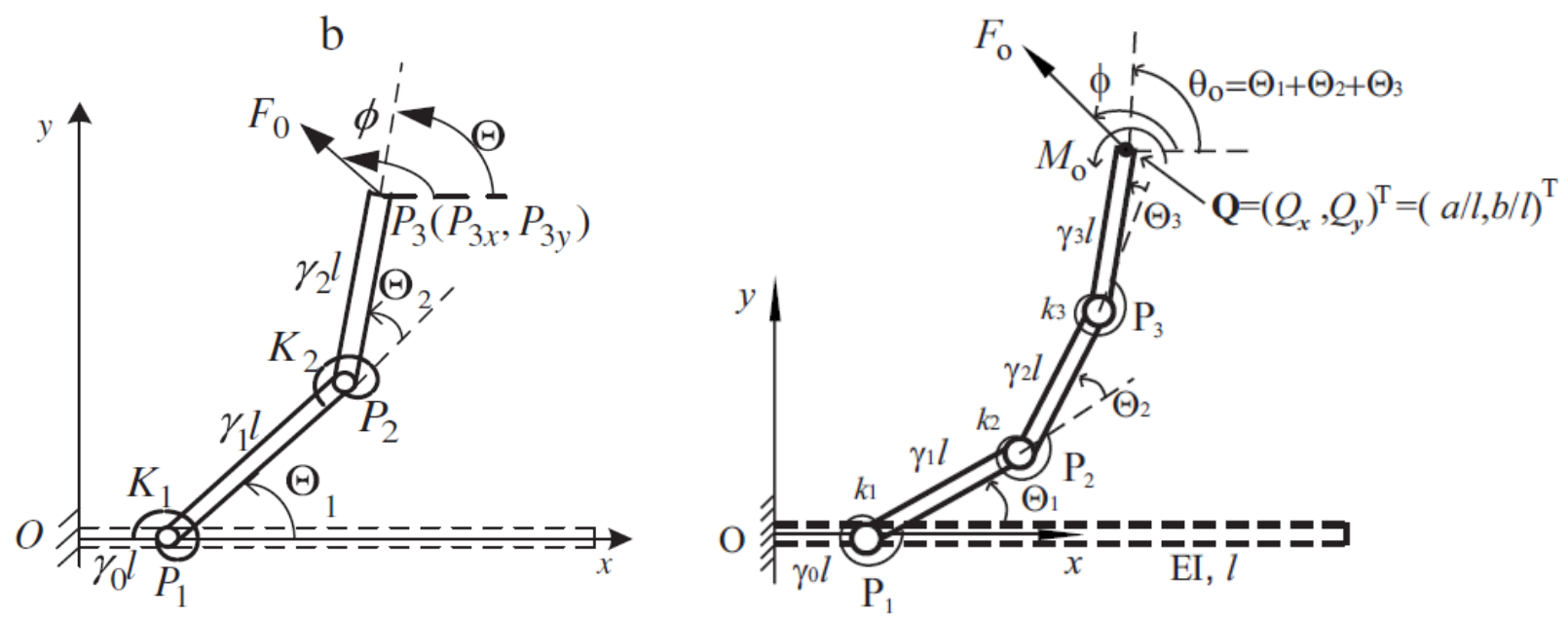

Figure 5 Pseudo-rigid-body models with multiple torsional springs. Source [17][18]

The inclusion of additional degrees of freedom (DOF) permits a reduction in the approximation error when compared with the conventional PRBM from Howell. Although PRBMs with multiple joints and torsional springs can outperform the PRBM proposed by Howell, the 1R PRBM is less complex and more user friendly.

Planar PRBM's used in various applications were reviewed to determine the state of the art in the compliant mechanisms area including human implants [19-20], bistable mechanisms [21-22], high precision devices [23], multiple degrees of freedom [24-25], and conceptual guidelines [26-29].

Lusk [30] quantified the uncertainty for planar PRBMs by solving the elastic beam equations as a function of arc length, $s$, along the beam's neutral axis. The system of differential equation is:

$$
\begin{gathered}
\frac{d \kappa}{d s}=\frac{F}{E I} \sin \left(\delta_{o}\right) \\
\frac{d \delta}{d s}=\kappa \\
\frac{d x}{d s}=\cos \left(\delta_{o}-\delta\right) \\
\frac{d y}{d s}=\sin \left(\delta_{o}-\delta\right)
\end{gathered}
$$


where $\kappa$ is the curvature of the deformed beam, $\delta_{o}$ is the angle that the neutral axis of the beam makes with applied force, and $\delta$ is the angle the applied force makes with the $x$-axis at a beam length equal to $s$. The horizontal and vertical coordinates of the neutral axis are represented by $x$ and $y$. Solving the equations in non-dimensional form includes a broader range of materials, cross sections and force combinations that represent infinite loading conditions. The characteristic length scale used to nondimensionalize the equations was,

$$
\lambda=\sqrt{\frac{E I}{\|F\|}}
$$

where $\|\mathrm{F}\|$ is the force magnitude, and EI, the flexural rigidity of the beam, is the product of Young's modulus, E, and the second moment of inertia in the bending axis, I. Equations (20)-(21) are nondimensionalized using characteristic length scale resulting in the following set of equations:

$$
\begin{gathered}
\frac{d \tilde{\kappa}}{d \tilde{s}}=\sin \left(\delta_{o}\right) \\
\frac{d \psi}{d \tilde{s}}=\tilde{\kappa} \\
\frac{d \tilde{x}}{d \tilde{s}}=\cos \left(\delta_{o}-\delta\right) \\
\frac{d \tilde{y}}{d \tilde{s}}=\sin \left(\delta_{o}-\delta\right)
\end{gathered}
$$

where the tilde accent indicates the variable is in non-dimensional form, e.g. $\tilde{\kappa}=\kappa \lambda$ and $\tilde{s}=s / \lambda$. In addition, when the beam is loaded with a force and no moment at the free end, the associated boundary condition is the nonexistence of curvature at the free end, i.e. $\tilde{\kappa}=0$. The solution is presented in Figure 6 which shows the phase portrait of the non-dimensional form of the elastica. The trajectories of the non- 
dimensional curvature, $\tilde{\kappa}$, versus, $\delta$, the angle the applied force makes with the x-axis at any beam length $\widetilde{\mathrm{s}}$ are symmetric about $\tilde{\mathrm{\kappa}}=0$ and $\delta=-180^{\circ}$.

Lusk stated "when obtaining solutions for all the cantilever beams with a force and no moment on the free end, it is more convenient to solve the differential equations in a frame of reference attached to the free end of the beam [30]”.

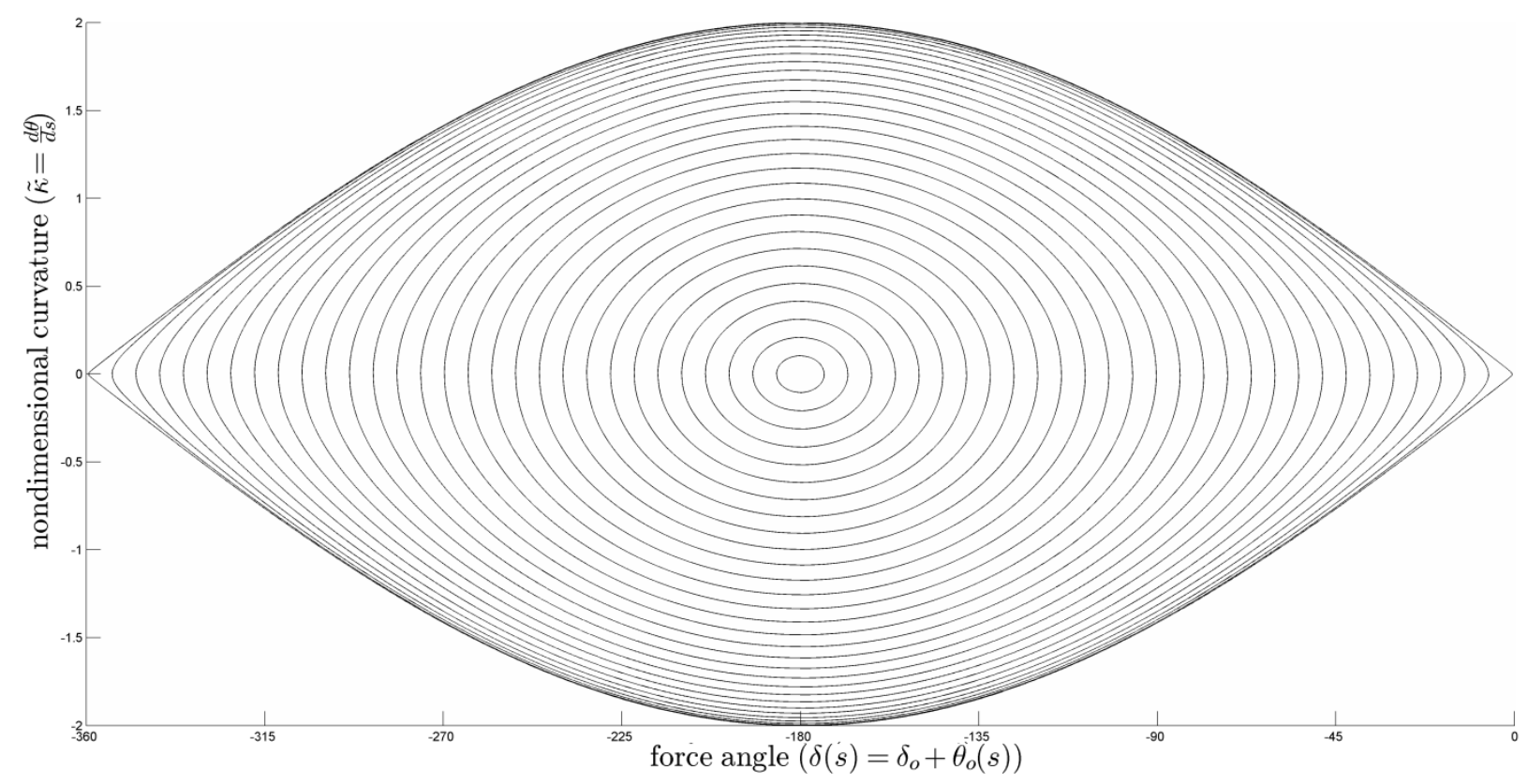

Figure 6 Phase portrait of the planar elastica. Adapted from [30]

Using this approach, the boundary condition related to the force angle, $\delta_{o}$ is invariant of the frame of reference attached to the fixed end. Figure 7, a detail of the phase-portrait, shows free end's reference frames represented by ellipses, and fixed end's reference frames represented by rectangles. Furthermore, a single red dotted trajectory represents a set cantilever beams with follower force whereas the blue dotted line represents a set of cantilever beams with non-follower force. When solutions are obtained using the fixed end frame, force increments require multiple numerically integrated trajectories because boundary conditions on the free end are changed. On the contrary, if solutions are obtained using 
the free end frame, force increments only require additional integration steps along a unique trajectory because boundary conditions on the free end are constant.

The result obtained from the planar beam system of differential equations include exact values for curvature at the fixed end, $x$ - and $y$-coordinates of the beam tip with respect to the fixed end of the beam and beam bending angle, $\theta_{o l}$. These results are presented in Figure 7-Figure 9. Furthermore, equation (7) is used to find the exact value of the characteristic radius factor, $\gamma$, and are presented in Figure 10. Relative error is calculated if the generic characteristic radius value of 0.85 was used in equations (4) and (5) compared to the exact values of $a$ and $b$ found on the solution and illustrated in Figure 11. These results are presented in phase portrait and contour plots using angle $\delta(s)$ as the abscissa. 


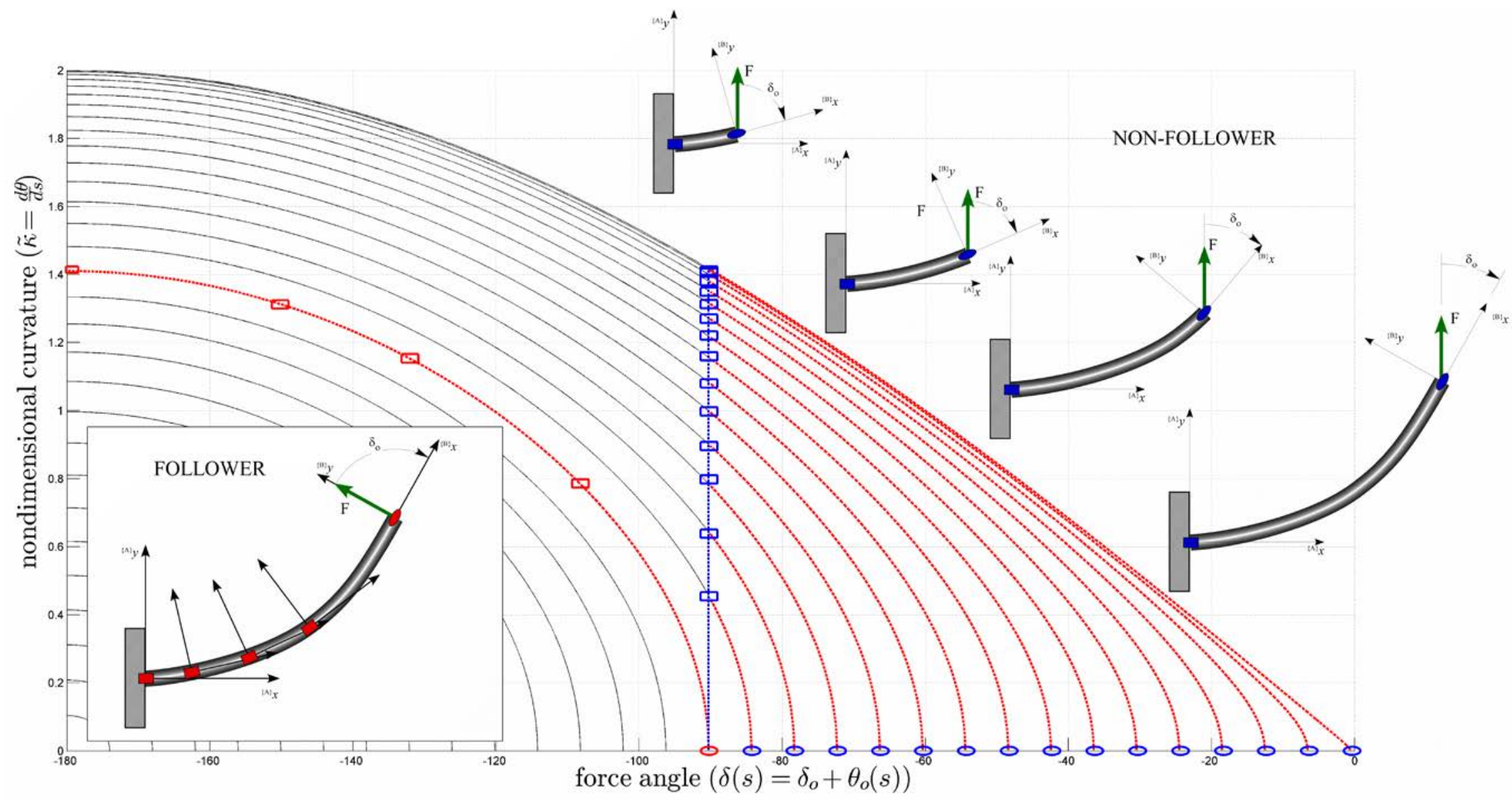

Figure 7 Phase-portrait showing non-dimensional curvature versus slope of the elastica. Adapted from [30].

17 


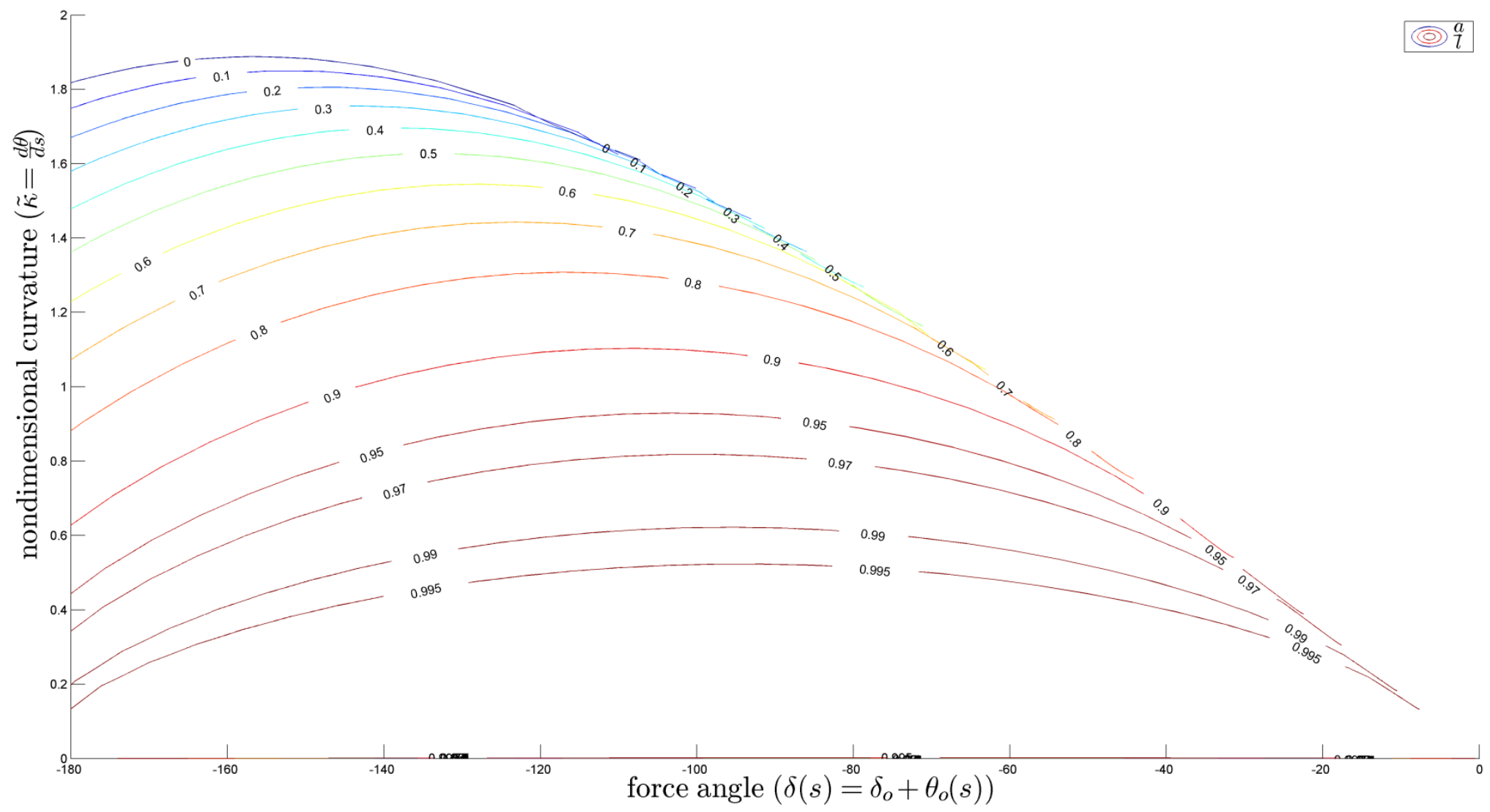

Figure 8 The x- coordinate of the beam tip. Adapted from [30]. 


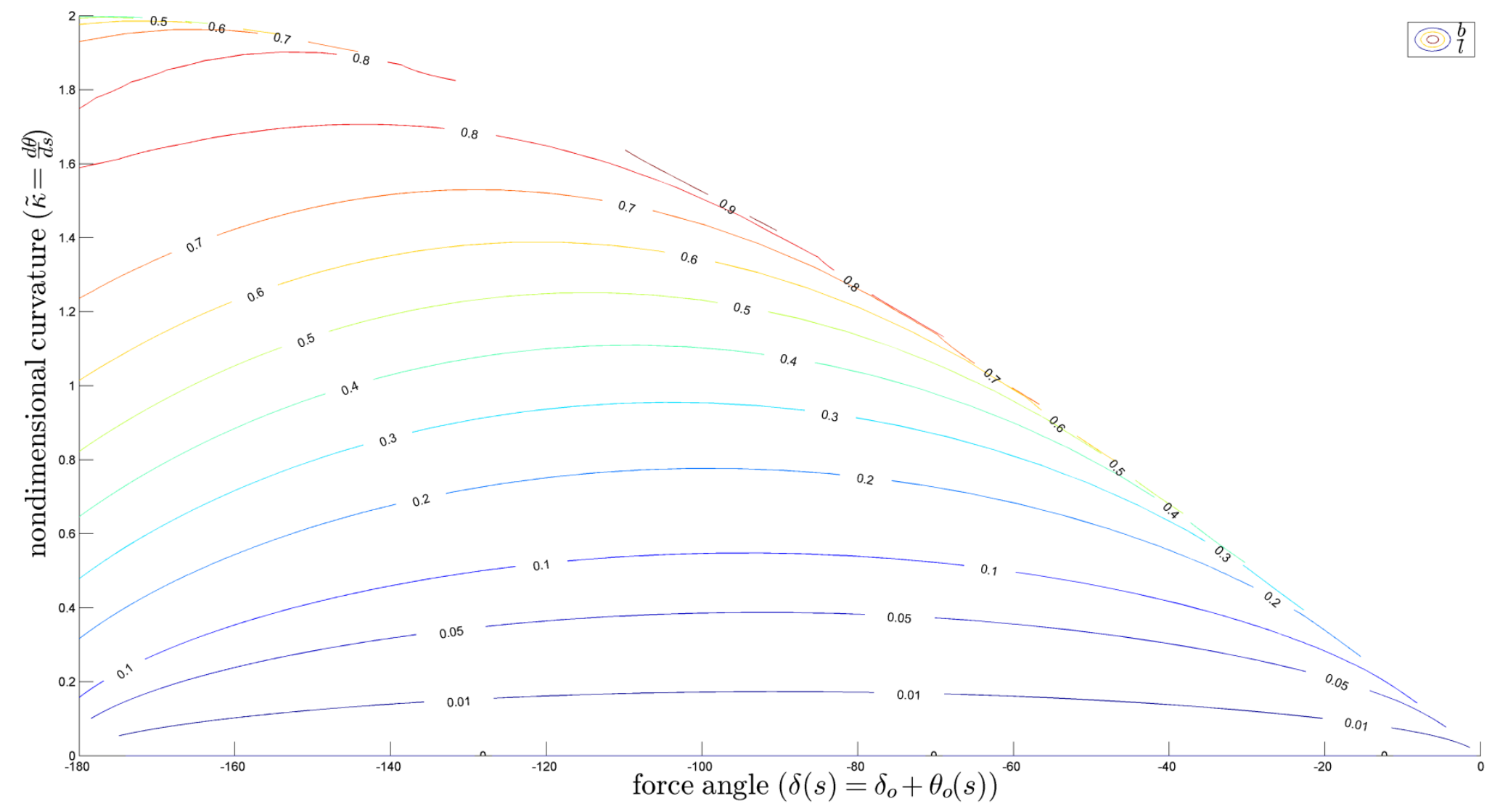

Figure 9 The y- coordinate of the beam tip. Adapted from [30]. 


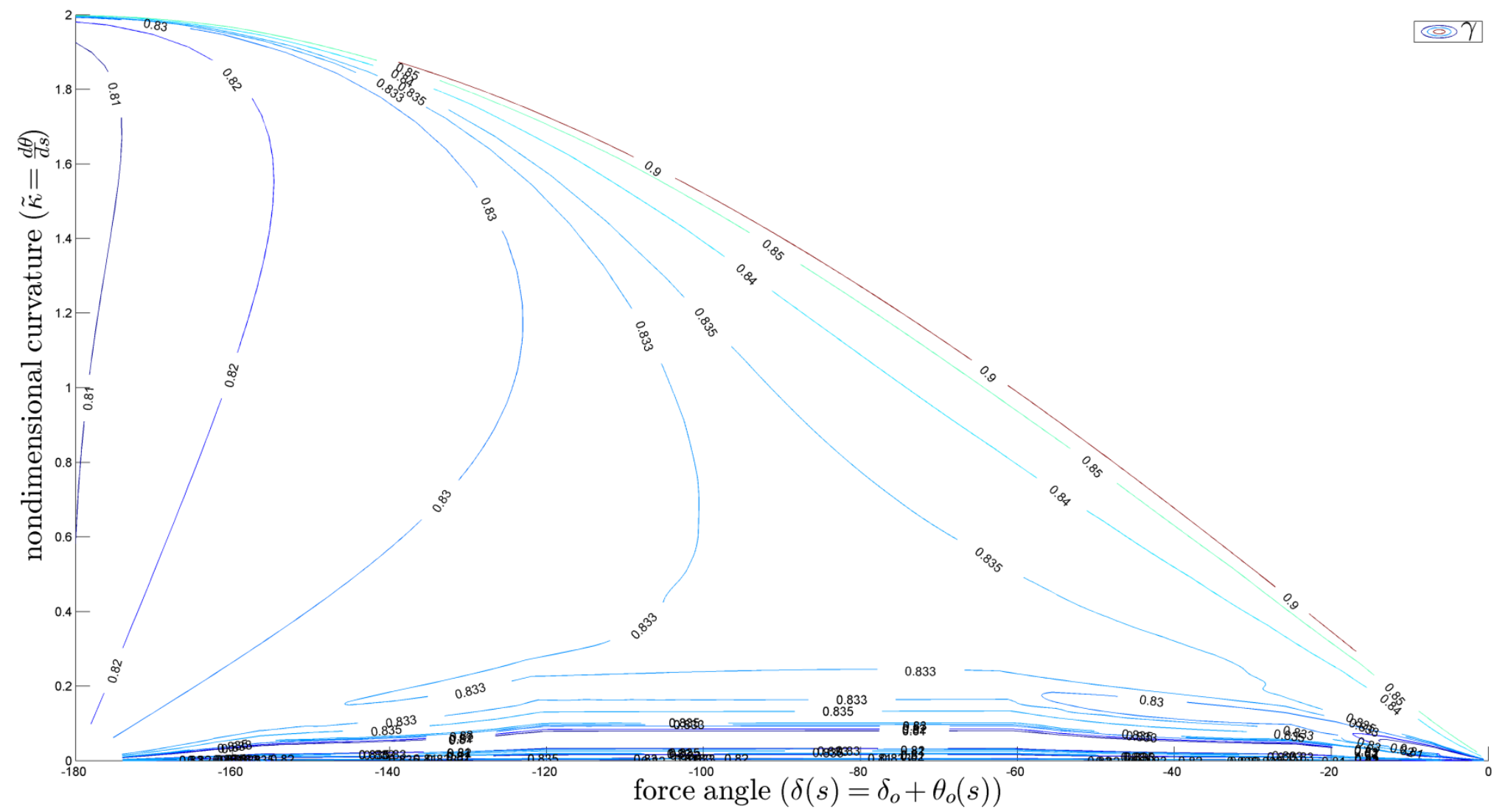

Figure 10 Exact values of characteristic radius factor using values of $a$ and $b$. Adapted from [30]. 


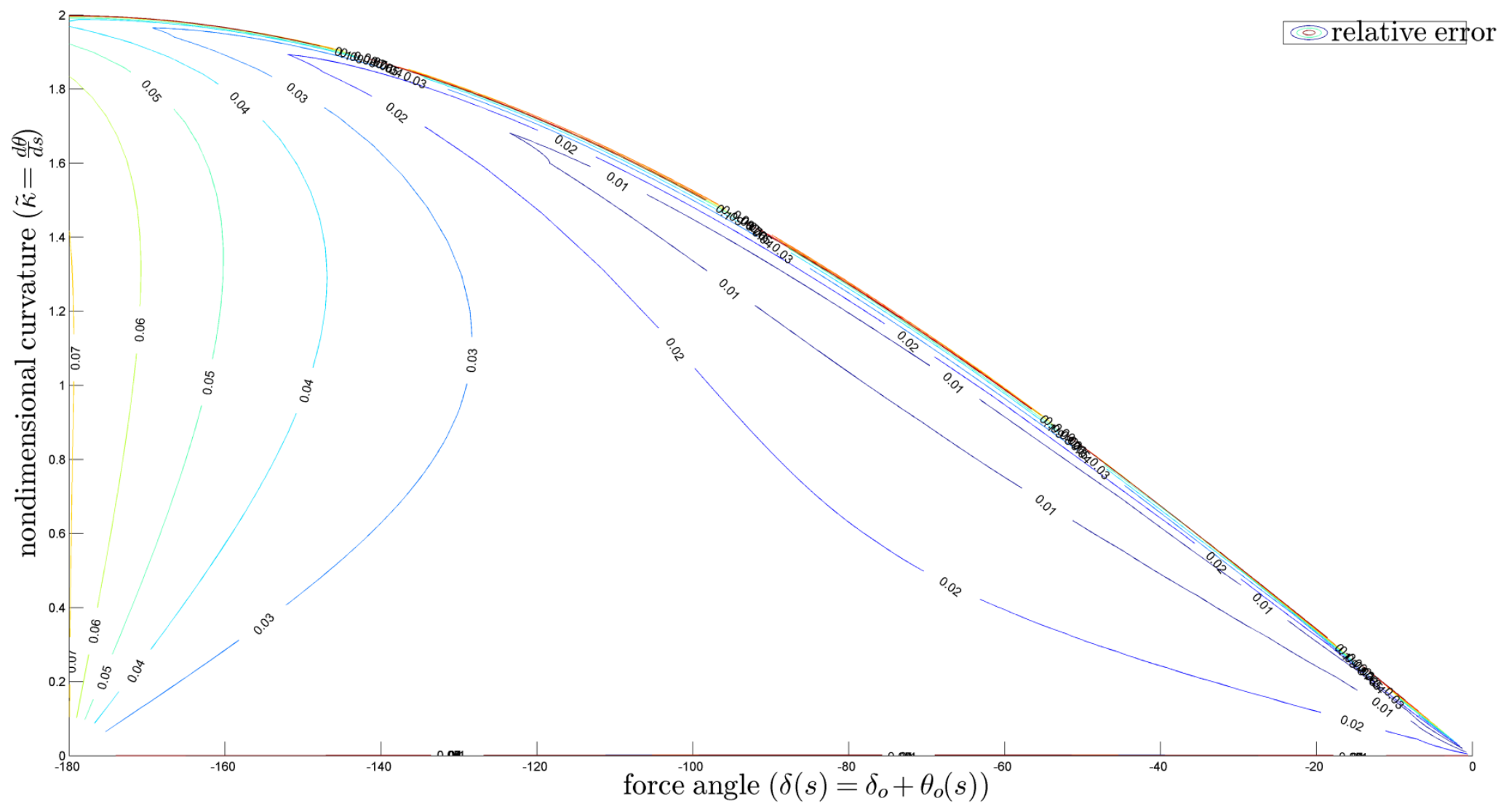

Figure 11 Relative error incurred from using $\gamma$ value of 0.85 in PRBM equations. Adapted from [30]. 


\section{CHAPTER 3: NUMERICAL INTEGRATION}

This section presents the governing equations for cantilever beams with arbitrary force and no moment at the free end, and the coordinate reference frames used in the process.

The governing equations that describe large, nonlinear deflections exhibited by cantilever beams in compliant mechanisms were described by Frisch-Fay [31] and adapted by Ramirez et al [32] using a system of nonlinear differential equations. However, in this dissertation the orientation of the reference frame attached at the beam free-end is rotated by $\pi$ radians in the local z-axis resulting in a sign change in the force directions terms. The frame of reference at the beam's free end is used to solve the system of differential equations for all the cantilever beams with an arbitrary force and no moment on the free-end. Chapter 2 discussed the advantages of solving the system of differential equations in the free end reference frame using follower forces.

\subsection{Governing Equations}

Equations (32)-(40) define the system of differential equations used to obtain the Euler angles set $(\psi, \theta$ and $\phi)$, curvatures $\left(\tau_{x}, \kappa_{y}\right.$ and $\left.\kappa_{z}\right)$ and the coordinates $(x, y$, and $z)$ of the initially straight cantilever beam loaded with an arbitrary follower force and no moment at the free end. An Euler angle set rotation,

${ }_{\{A\}}^{\{H}{ }_{X} R_{X Z X}(\psi, \theta, \phi)$, was used to describe the rotation from the reference free end frame, $\{H\}$, to the relative frame of the fixed end, $\{\mathrm{A}\}$, of a beam loaded with a follower force and no moment at the free end. Reference frame $\{\mathrm{A}\}$ changes at each integration step along a neutral axis trajectory of a beam loaded with a non-follower force. Figure 12 and Figure 13 show the location and orientation of the frames at the beam ends with respect to the free end and fixed end respectively. 


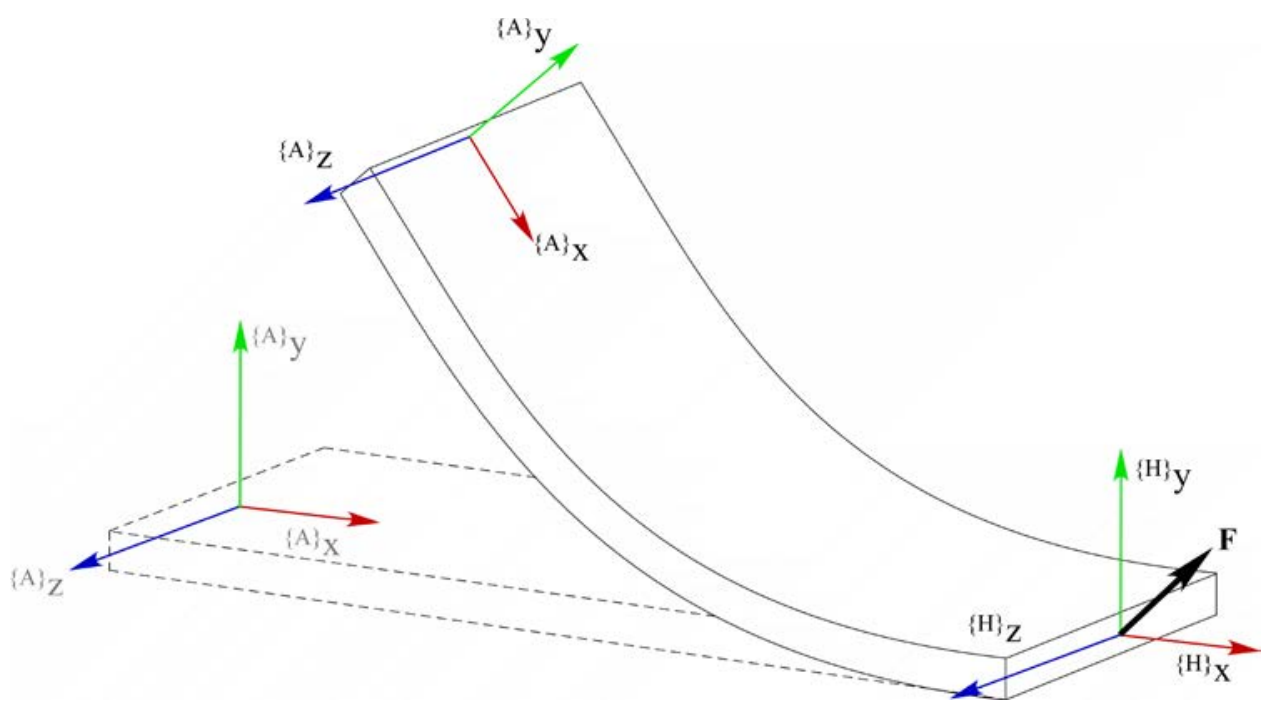

Figure 12 Deflected beam with frame $\{\mathrm{H}\}$ as reference

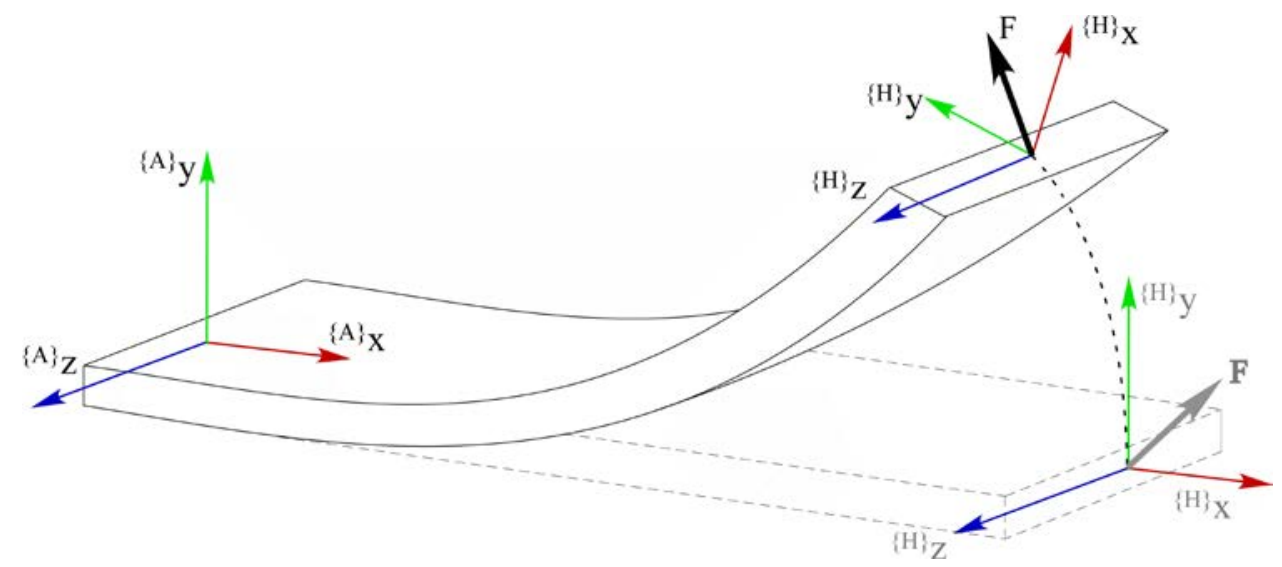

Figure 13 Deflected beam with frame $\{\mathrm{A}\}$ as reference

The angles of inclination of follower forces with respect to the deformed free end neutral axis of the beam remain constant during deflection (i.e. ${ }^{\{\mathrm{H}\}} \overrightarrow{\mathrm{F}}$ is constant). The components of the spatial follower force in the $\{\mathrm{H}\}$ frame can be obtained using the following expressions:

$$
\begin{gathered}
{ }^{\{H\}} F_{x}=\|F\| \sin \eta \cos \xi \\
{ }^{\{H\}} F_{y}=\|F\| \sin \eta \sin \xi \\
{ }^{\{H\}} F_{z}=\|F\| \cos \eta
\end{gathered}
$$


where $\eta$ and $\xi$ are angles of a spherical coordinate system that define the orientation of the follower force with respect of frame $\{H\}$ as shown in Figure 14. Due to symmetry of the beam this dissertation only focuses on the subspace where $\eta=\left[90^{\circ}-180^{\circ}\right]$ and $\xi=\left[0^{\circ}-180^{\circ}\right]$, which is one quarter of the sphere of possible force directions, and reflect the fact that a rectangle has 2 axes of symmetry (4 symmetric regions).

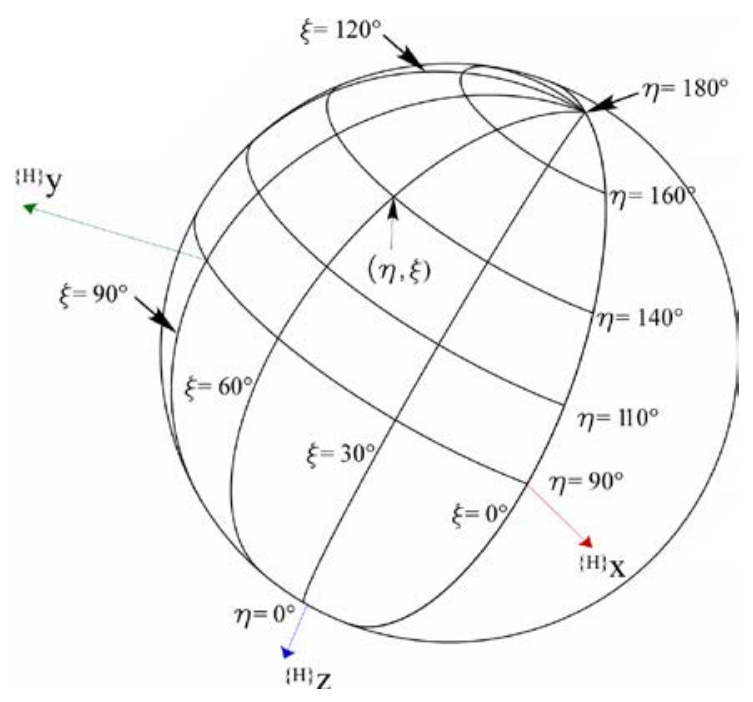

Figure 14 Force direction described with spherical coordinates

The governing equations are:

$$
\begin{gathered}
\frac{{ }^{\{H\}} d \phi}{d s}={ }^{\{A\}} \tau_{x}+{ }^{\{A\}} \kappa_{y} \cos \phi \frac{\cos \theta}{\sin \theta}-{ }^{\{A\}} \kappa_{z} \sin \phi \frac{\cos \theta}{\sin \theta} \\
\frac{{ }^{\{H\}} d \theta}{d s}={ }^{\{A\}} \kappa_{y} \sin \phi+{ }^{\{A\}} \kappa_{z} \cos \phi \\
\frac{{ }^{\{H\}} \frac{d \psi}{d s}}{d A}=-{ }^{\{A\}} \kappa_{y} \frac{\cos \phi}{\sin \theta}+{ }^{\{A\}} \kappa_{z} \frac{\sin \phi}{\sin \theta} \\
\frac{{ }^{\{A\}}}{d s}=\frac{1}{G{ }^{\{A\}} I_{x x}}\left(\left(E^{\{A\}} I_{y y}-E^{\{A\}} I_{z z}\right)\{A\} \kappa_{y}{ }^{\{A\}} \kappa_{z}\right) \\
\frac{d \kappa_{y}}{d s}=\frac{1}{E^{\{A\}} I_{y y}}\left(\left(E^{\{A\}} I_{z z}-G^{\{A\}} I_{x x}\right){ }^{\{A\}} \tau_{x}{ }^{\{A\}} \kappa_{z}+{ }^{\{A\}} F_{z}\right)
\end{gathered}
$$




$$
\begin{aligned}
& \frac{{ }^{\{A\}} d \kappa_{z}}{d s}=\frac{1}{E^{\{A\}} I_{z z}}\left(\left(G^{\{A\}} I_{x x}-E^{\{A\}} I_{y y}\right){ }^{\{A\}} \tau_{x}{ }^{\{A\}} \kappa_{y}-{ }^{\{A\}} F_{y}\right) \\
& { }^{\{H\}} \frac{d x}{d s}=\cos \theta \\
& { }^{\{H\}} \frac{d y}{d s}=\cos \psi \sin \theta \\
& { }^{\{H\}} \frac{d z}{d s}=\sin \psi \sin \theta
\end{aligned}
$$

where ${ }^{\{A\}} I_{x x},{ }^{\{A\}} I_{y y}$ and ${ }^{\{A\}} I_{z z}$ are the second moments of area with respect to the $x-, y$ - and $z$-axis in the \{A\} frame. The ODE45 function in Matlab numerically integrates the system of differential equations from $s=0$, at the free end, to $s=-l$, at fixed end, in frame $\{\mathrm{H}\}$. Numerical integration of the system solves for Euler angles, the position coordinates expressed in the coordinate system $\{H\}$, and curvatures expressed in the coordinate system $\{\mathrm{A}\}$. In order to express force and position coordinates in the $\{\mathrm{A}\}$ frame, they must be pre-multiplied by a rotation matrix using negative of the Euler angle set in the reverse order.

$$
\begin{gathered}
{ }^{\{A\}} \vec{F}={ }_{\{H\}}^{\{A\}} R_{X Z X}(-\phi,-\theta,-\psi){ }^{\{H\}} \vec{F} \\
{ }^{\{A\}} \vec{X}=-{ }_{\{H\}}^{\{A\}} R_{X Z X}(-\phi,-\theta,-\psi)^{\{H\}} \vec{X}
\end{gathered}
$$

Equations (32)-(34) are found from the differential relationship between the Euler angles and the curvatures. If a different Euler angle set is selected, the relationship would be different. Equations (35)(37) are derived from the 3 -D versions of the Bernoulli-Euler equations, $\vec{M}=E I \vec{k}$, by taking its derivative with respect to the arc-length $s$. Equations (38)-(40) describe the $\{\mathrm{H}\}$ frame components of $\left.{ }^{\{A}\right\} \hat{x}$ unit vector for a particular integration step, in other words they give the direction of the neutral axis as it lengths away from the free-end. 


\section{CHAPTER 4: THREE DIMENSIONAL PSEUDO-RIGID-BODY MODEL}

This chapter describes a novel PRBM derived from the solutions of the spatial large deflection equations, which is the first PRBM that approximates the behavior of an initially straight cantilever beam with rectangular cross section loaded with an arbitrary force and no moment. This PRBM is able to predict the position and orientation of the neutral axis of the beam in addition to the moments at the fixed end.
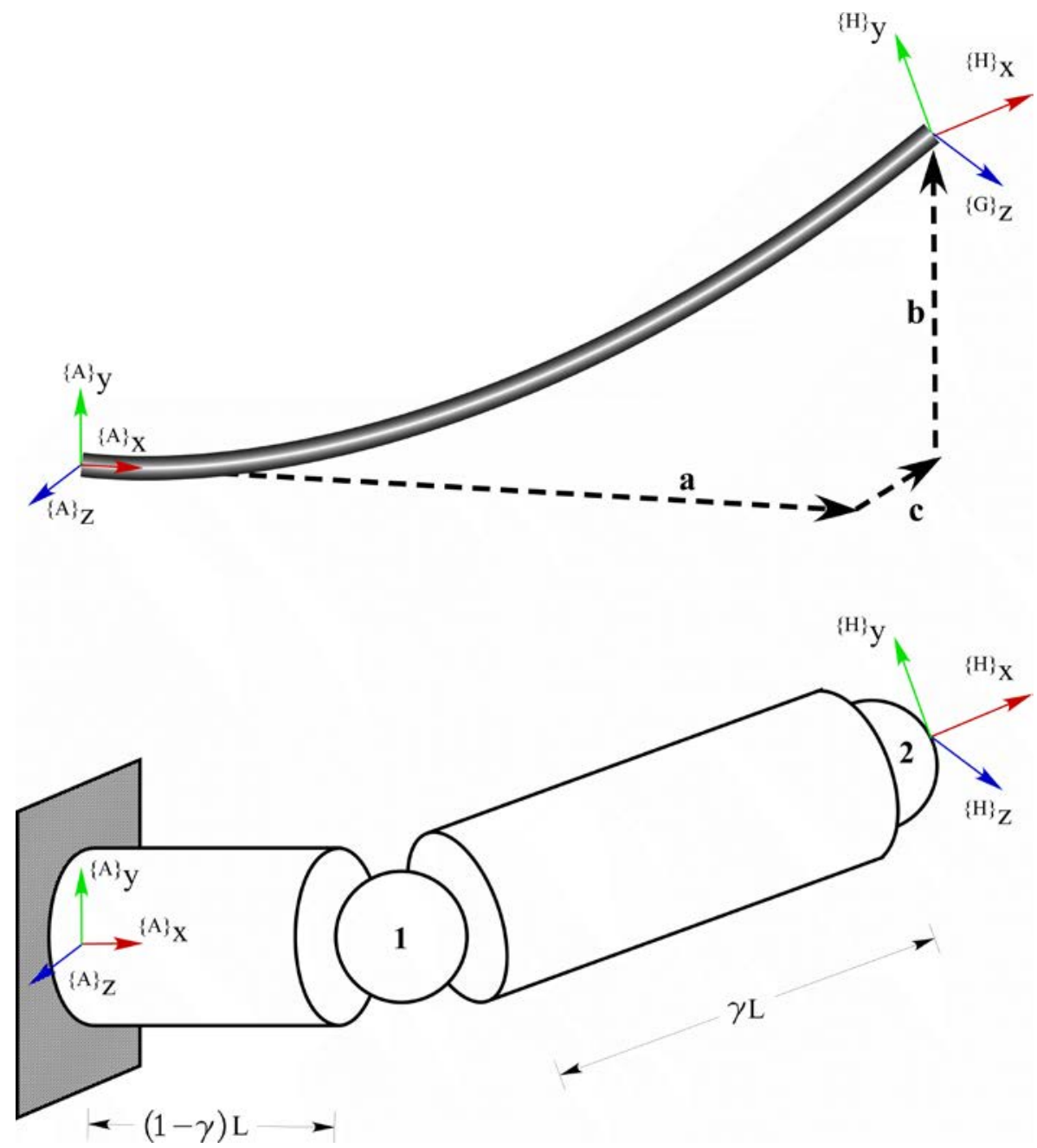

Figure 15 General 3-D PRBM. 
A general 3-D PRBM consists of two rigid links and a thin end cap. The first rigid link is fixed to ground in one end and connects to the second rigid link with the spherical joint 1 . The second rigid link connects to a thin $(L=0)$ end cap with the spherical joint 2 . The end cap is a plane whose orientation is identical to the orientation of the free end of the compliant beam. Figure 15 illustrates the concept described above.

\subsection{Position Equations}

Numerical integration provides the position coordinates of the neutral axis of the beam from the fixed end with respect to the free-end of the beam. However, designers are used to express the position coordinates of the deflected beam using the frame at the fixed end. Thus, Equation (42) was used to map position coordinates from $\{\mathrm{H}\}$ to $\{\mathrm{A}\}$. The position coordinates of the neutral axis at the free-end $(a, b$ and $c$ ), in the $\{\mathrm{A}\}$ frame, are given by:

$$
\begin{gathered}
a=l-\gamma l(1-\cos \Theta) \\
b=\gamma l \sin \Theta \cos \Phi \\
c=\gamma l \sin \Theta \sin \Phi
\end{gathered}
$$

where $l$ is the beam length, $\gamma$ is the characteristic radius factor, $\Theta$ is the bending pseudo-rigid-body angle, and $\Phi$ is the twisting pseudo-rigid-body angle. Angle $\Phi$ is the rotation angle of the first rigid link with respect of the $\mathrm{X}$-axis in frame $\{\mathrm{A}\}$.

\subsection{Angle Equations}

Because $a, b$ and $c$ can be found from numerical integration of equations (32)-(40), parameters of the model, $\gamma, \Theta$ and $\Phi$, can be obtained for every loading condition analytically solving equations (43)(45): $\{A\}\}=R_{X Z}(\Phi, \Theta)$ is a rotation from the $\{\mathrm{A}\}$ frame to the $\{\mathrm{D}\}$ frame at the characteristic pivot which take place in the spherical joint 1, depicted in Figure 16. 


$$
\begin{gathered}
\Phi=\tan ^{-1}\left(\frac{c}{b}\right) \\
\gamma=\frac{\left(\frac{b}{l}\right)^{2}+\left(\frac{c}{l}\right)^{2}+\left(1-\frac{a}{l}\right)^{2}}{2\left(1-\frac{a}{l}\right)} \\
\Theta=\tan ^{-1}\left(\frac{\frac{b}{l \cos \Phi}}{\left(\frac{a}{l}-1\right)+\gamma}\right)
\end{gathered}
$$

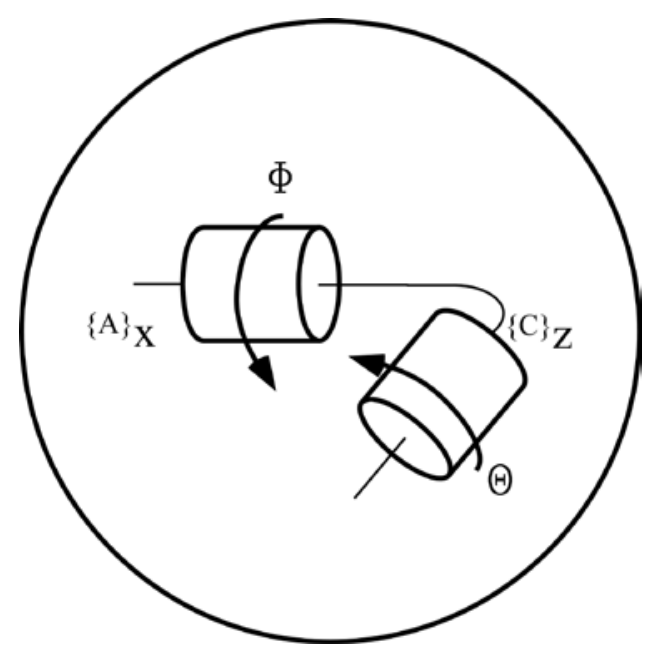

Figure 16 Spherical joint 1

The orientation of the free end of the beam expressed in the $\{\mathrm{A}\}$ frame is determined by the rotation matrix ${ }_{\{H\}}^{\{A\}} R_{X Z X}(-\phi,-\theta,-\psi)$. In order to ensure that the end cap orientation will always be identical to the beam's free end orientation, the second spherical joint is allowed to perform a XZX rotation, $R_{X Z X}(\Sigma, \Omega, \Psi)$. Since rotation matrices are orthogonal, their inverse is equal to the transpose (e.g. $\left.R^{-1}=R^{T}\right)$. Thus,

$$
\begin{gathered}
\{A\} R_{X Z X}(-\phi,-\theta,-\psi)={ }_{\{D\}}^{\{A\}} R * R_{X Z X}(\Sigma, \Omega, \Psi) \\
\{D\} R_{X Z X}(\Sigma, \Omega, \Psi)=R_{Z}^{-1}(\Theta) R_{X}{ }^{-1}(\Phi)_{\{H\}}^{\{A\}} R_{X Z X}(-\phi,-\theta,-\psi)
\end{gathered}
$$




$$
{ }_{\{H\}}^{\{D\}} R_{X Z X}(\Sigma, \Omega, \Psi)=R_{Z}^{T}(\Theta) R_{X}^{T}(\Phi)_{\{H\}}^{\{A\}} R_{X Z X}(-\phi,-\theta,-\psi)
$$

Equations (49)-(51) is a 3x3 system of equations that is solved as follows.

$$
\begin{gathered}
\Omega=\tan ^{-1} \frac{\sqrt{\left(\begin{array}{l}
\{D\} \\
\{H\}
\end{array} R_{X Z X}(2,1)\right)^{2}+\left(\left(\begin{array}{l}
\{D\} \\
\{H\}
\end{array} R_{X Z X}(3,1)\right)\right)^{2}}}{\left(\begin{array}{l}
\{D\} \\
\{H\}
\end{array} R_{X Z X}(1,1)\right)} \\
\Sigma=\tan ^{-1} \frac{\frac{\{D\}}{\sin \Omega} R_{X Z X}(3,1)}{\frac{\{D\}}{2 H\}} R_{X Z X}(2,1)} \\
\sin \Omega \\
\Psi=\tan ^{-1} \frac{\frac{\{H\}}{\sin \Omega}}{\frac{-\{D\} R_{X Z X}(2,1)}{\sin \Omega}}
\end{gathered}
$$

We can now establish a relationship between 3-D PRBM angles $\Phi, \Theta$ and $\Psi$ and Euler angles $\psi, \theta$ and $\phi$.

$$
\begin{gathered}
\phi=c_{\phi} \Phi \\
\theta=c_{\theta} \Theta \\
\psi=c_{\psi} \Psi
\end{gathered}
$$

where $c_{\phi}, c_{\theta}$ and $c_{\psi}$ are called parametric angle coefficients. The previously defined coordinate rotations $(\Sigma, \Omega, \Psi)$ take place in the second spherical joint illustrated in Figure 17. 


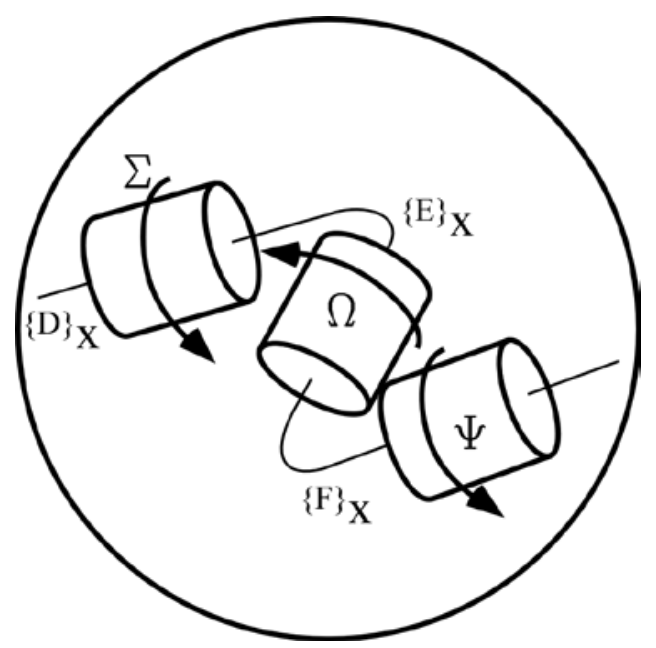

Figure 17 Spherical joint 2.

\subsection{Virtual Work}

The stiffness of the beam was calculated using the method of virtual work. The general 3-D PRBM has two torsional springs associated with angles $Y$ and $\Theta$. In addition, the twisting pseudo-rigidbody angle, $\Phi$, includes two distinct physical effects as illustrated in Figure 18.

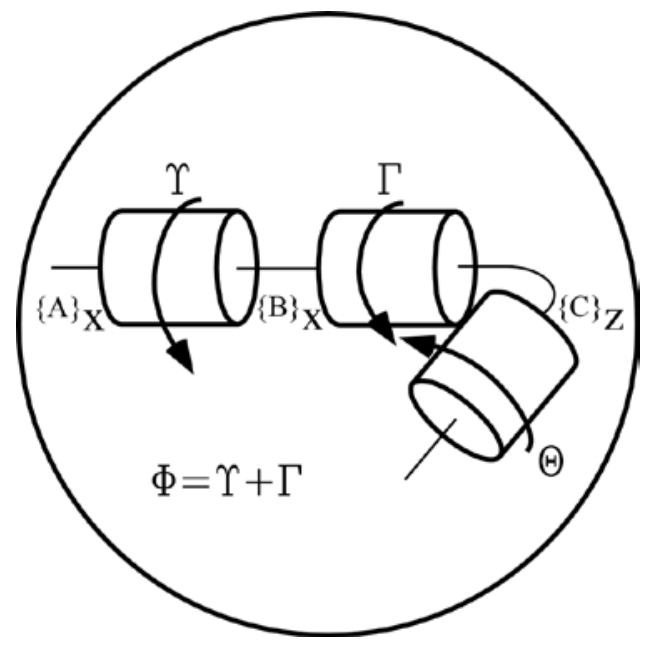

Figure 18 Spherical joint 1 with twisting and orientation angles

One effect is the twisting of the first rigid link in the model whereas the other effect describes the orientation of the bending direction relative to the beam's principal axes. Thus the angle relationship can be defined as, 


$$
\Phi=\Upsilon+\Gamma
$$

or more precisely,

$$
{ }_{\{C\}}^{\{A\}} R_{X}(\Phi)={ }_{\{B\}}^{\{A\}} R_{X}(Y) *{ }_{\{C\}}^{\{B\}} R_{X}(\Gamma)
$$

where $Y$ is the angle of twist of the first rigid link, and $\Gamma$ is the orientation of the bending direction relative to the principal moments of area of the beam.

The stiffness of the beam under force loading is calculated from the potential energy of the two torsional springs, $\Upsilon$ and $\Theta$, located at the first spherical joint. The torsional potential energy of the first rigid segment is given by:

$$
V_{T}=\frac{1}{2} * \frac{\{C\}}{(1-\gamma) L} I_{x x} Y^{2}
$$

which is the classic torsion potential energy for a beam of length $(1-\gamma) L$. Similarly,

$$
V_{B}=\frac{1}{2} * \frac{\{C\} I_{z z} E}{L} \gamma K_{\Theta} \Theta^{2}
$$

where $V_{B}$ is similar to Howell's expression for bending potential energy of a 2-D compliant beam, with the distinction that ${ }^{\{C\}} I_{z z}$ is expressed in the $\{C\}$ frame. This distinction is crucial because it correctly captures the bending stiffness about a non-principal stiffness axes. Because $\Upsilon$ represents a physical twisting of the beam's cross-section, ${ }^{\{A\}} I_{y y}={ }^{\{B\}} I_{y y}$ and ${ }^{\{A\}} I_{z z}={ }^{\{B\}} I_{z z}$, (i.e. $\{\mathrm{B}\}$ frame is a principal 
stiffness frame). Thus using the transformation rule for 2-D second order tensors (Mohr's circle) and coordinate rotation angle $\Gamma$, we state that

$$
\begin{gathered}
\bar{I}=\frac{{ }^{\{B\}} I_{y y}+{ }^{\{B\}} I_{z z}}{2} \\
I_{R}=\frac{\{B\} I_{y y}-{ }^{\{B\}} I_{z z}}{2} \\
{ }^{\{C\}} I_{z z}=\bar{I}-I_{R} \cos (2 \Gamma)
\end{gathered}
$$

It is important to mention that $\bar{I}$ and $I_{R}$ are invariant under rotations about ${ }^{\{B\}} x$, so they can be used in $\{A\},\{B\}$ and $\{C\}$ frames. The virtual work equations are given by

$$
\sum_{i} \vec{F}_{l} \cdot \delta \overrightarrow{X_{l}}-\sum_{k} \frac{\partial V}{\partial q_{k}} \delta q_{k}=0
$$

where $\vec{X}=X\left(q_{k}\right)$ and $q_{k}=(\Upsilon, \Gamma, \Theta)$. The position of the free end is defined by,

$$
\vec{X}=L(1-\gamma)^{\{A\}} \hat{x}+\gamma L^{\{D\}} \hat{x}
$$

because ${ }^{\{\mathrm{A}\}} \hat{x}$ is constant, and ${ }^{\{D\}} \hat{x}$ is a rotating vector, $d \vec{X}$ becomes:

$$
\begin{gathered}
d \vec{X}=\gamma L\left(d \Phi^{\left.\{C\}_{\hat{x}}+d \Theta^{\{D\}} \hat{z}\right) \times{ }^{\{D\}} \hat{x}}\right. \\
{\left[\begin{array}{c}
{ }^{\{C\}_{x}} \\
\{C\}_{y} \\
\{C\}_{z}
\end{array}\right]=\left[\begin{array}{ccc}
\cos \Theta & \sin \Theta & 0 \\
-\sin \Theta & \cos \Theta & 0 \\
0 & 0 & 1
\end{array}\right]\left[\begin{array}{c}
\{D\} \\
\{D\} \\
\{D\}_{z}
\end{array}\right]=\left[\begin{array}{c}
{ }^{\{D\}} x \cos \Theta+{ }^{\{D\}} y \sin \Theta \\
-{ }^{\{D\}} x \sin \Theta+{ }^{\{D\}} y \cos \Theta \\
\{D\}_{z}
\end{array}\right]}
\end{gathered}
$$




$$
\begin{gathered}
d \vec{X}=\gamma L\left|\begin{array}{ccc}
{ }^{\{D\}} x & \{D\}_{y} & \{D\} z \\
d \Phi \cos \Theta & d \Phi \sin \Theta & d \Theta \\
1 & 0 & 0
\end{array}\right|=\gamma L\left[\begin{array}{c}
0^{\{D\}} x \\
d \Theta^{\{D\}} y \\
d \Phi \sin \Theta^{\{D\}} z
\end{array}\right] \\
d \vec{X}=\gamma L\left((d r+d \Gamma) \sin \Theta^{\{D\}} \hat{z}+d \Theta^{\{D\}} \hat{y}\right)
\end{gathered}
$$

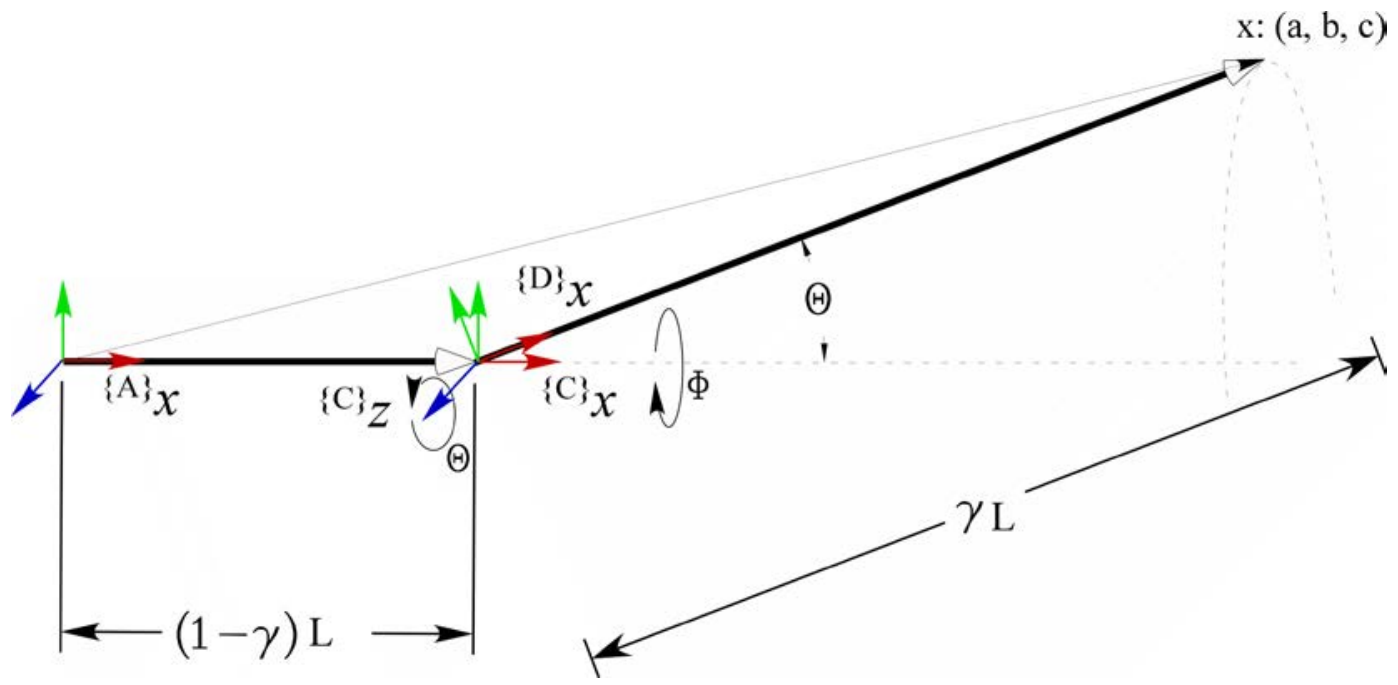

Figure 19 Beam tip position

To facilitate the dot product in equation (65) we express the force in the $\{\mathrm{D}\}$ frame using the following rotation:

$$
{ }^{\{D\}} \vec{F}=R_{Z}^{T}(\Theta) R_{X}{ }^{T}(\Phi)^{\{A\}} \vec{F}
$$

The first term of equation (65) becomes:

$$
\vec{F} \cdot d \vec{X}=\gamma L d \Theta^{\{D\}} F_{y}+\gamma L(d \Upsilon+d \Gamma) \sin \Theta^{\{D\}} F_{z}
$$


Partial differentiation with respect to $\Upsilon, \Gamma$ and, $\Theta$ of equations (60) and (61) yield

$$
\begin{gathered}
-\frac{d V_{T}}{d \gamma} \delta \gamma=-\frac{{ }^{\{C\}} I_{x x} G}{(1-\gamma) L} \gamma \delta \gamma \\
-\frac{d V_{B}}{d \Gamma} \delta \Gamma=-\gamma K_{\Theta} \frac{E}{L} I_{R} \Theta^{2} \sin (2 \Gamma) \delta \Gamma \\
-\frac{d V_{B}}{d \Theta} \delta \Theta=-\gamma K_{\Theta} \frac{E}{L}\left(\bar{I}-I_{R} \cos (2 \Gamma)\right) \Theta \delta \Theta
\end{gathered}
$$

Substitution of equations (71)-(73) into (65) yield,

$$
\begin{gathered}
\delta \gamma:\left(\gamma L^{\{D\}} F_{z} \sin \Theta-\frac{\{C\} I_{x x} G}{(1-\gamma) L} \gamma\right)=0 \\
\delta \Gamma:\left(\gamma L^{\{D\}} F_{z} \sin \Theta-\gamma K_{\Theta} \frac{E}{L} I_{R} \Theta^{2} \sin (2 \Gamma)\right)=0 \\
\delta \Theta:\left(\gamma L^{\{D\}} F_{y}-\gamma K_{\Theta} \frac{E}{L}\left(\bar{I}-I_{R} \cos (2 \Gamma)\right) \Theta\right)=0
\end{gathered}
$$

\subsection{Moment Equations}

Figure 20 shows the moments at the characteristic pivot due to $\vec{F}$ with no moment applied at the end of the beam.

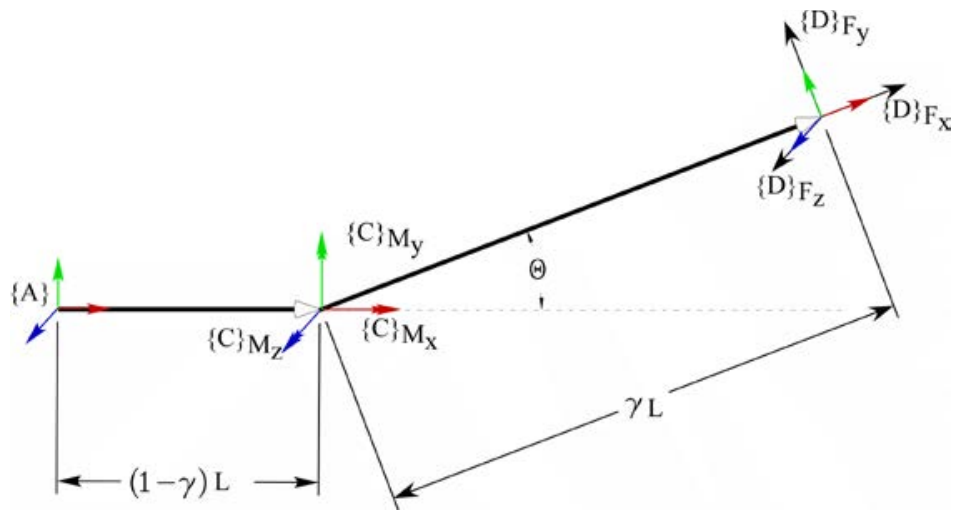

Figure 20 Moments at characteristic pivot 
Moments are expressed in the $\{\mathrm{C}\}$ frame using the following equations:

$$
\begin{gathered}
{ }^{\{C\}} M_{x}=\gamma L^{\{D\}} F_{z} \sin \Theta \\
{ }^{\{C\}} M_{y}=-\gamma L^{\{D\}} F_{z} \cos \Theta=-{ }^{\{C\}} M_{x} \cot \Theta \\
{ }^{\{C\}} M_{z}=\gamma L^{\{D\}} F_{y}
\end{gathered}
$$

Replacing equations (77) and (79) into (74) and (76) yields,

$$
\begin{gathered}
{ }^{\{C\}} M_{x}=\frac{{ }^{\{C\}} I_{x x} G}{(1-\gamma) L} K_{Y} \gamma \\
{ }^{\{C\}} M_{z}=\gamma K_{\Theta} \frac{E}{L}\left(\bar{I}-I_{R} \cos (2 \Gamma)\right) \Theta
\end{gathered}
$$

This moment can be also expressed in the $\{\mathrm{A}\}$ frame attached to the fixed end with the following expression:

$$
{ }^{\{A\}} \vec{M}={ }_{\{C\}}^{\{A\}} R_{X}(\Phi)^{\{C\}} \vec{M}+{ }^{\{A\}}(1-\gamma) L_{x} \times{ }^{\{A\}} \vec{F}
$$

The moments in frame $\{\mathrm{A}\}$ using 3-D PRBM parameters, can be compared with results from FEA or numerical integration. The moments in frame $\{\mathrm{A}\}$ from numerical integration can be expressed as follows,

$$
\begin{aligned}
& { }^{{ }^{A A} M_{x}}={ }^{\{A\}} I_{x x} G^{\{A\}} \tau_{x} \\
& { }^{\{A\}} M_{y}={ }^{\{A\}} I_{y y} E^{\{A\}} \kappa_{y} \\
& { }^{\{A\}} M_{z}={ }^{\{A\}} I_{z z} E^{\{A\}} \kappa_{z}
\end{aligned}
$$




\subsection{Validation for Planar Loading (2-D) and Axisymmetric Beam}

The following section demonstrates that the proposed 3-D PRBM is also valid for $\mathrm{xz}$ and $\mathrm{xy}$ planar loading, and axisymmetric beams. Since virtual work equations require expression for ${ }^{\{D\}} F_{z}$, the force component in the z-axis is calculated using equation (69).

$$
{ }^{\{D\}} F_{z}={ }^{\{A\}} F_{z} \cos \Phi-{ }^{\{A\}} F_{y} \sin \Phi
$$

For the xz planar case, ${ }^{\{A\}} F_{y}=0$ and $b=0$,

$$
\Phi=\tan ^{-1}\left(\frac{c}{b}\right)=\tan ^{-1}\left(\frac{c}{0}\right)= \pm \frac{\pi}{2}
$$

with ${ }^{\{A\}} F_{y}=0$ and $\cos (\Phi)=0$ equation (86) reduces to

$$
{ }^{\{D\}} F_{z}=0
$$

Replacing ${ }^{\{D\}} F_{z}$ into equation (74), we obtain

$$
\frac{{ }^{\{C\}} I_{x x} G}{(1-\gamma) L} \gamma=0
$$

it is required that $\frac{{ }^{\{C\}} I_{x x} G}{(1-\gamma) L} \neq 0$, thus

$$
r=0
$$


substitution on equation (58) yields,

$$
\begin{gathered}
\Phi=\Upsilon+\Gamma \\
\Gamma= \pm \frac{\pi}{2}
\end{gathered}
$$

Equation (76) simplifies to:

$$
\left(\gamma L^{\{D\}} F_{y}-\gamma K_{\Theta} \frac{E}{L}{ }^{\{A\}} I_{y y} \Theta\right)=0
$$

which is similar to standard planar PRBMs. The previous results show that the beam will not experience twist and twisting pseudo-rigid-body angle, $\Phi$, will be $\pm 90^{\circ}$ depending on the sign of ${ }^{\{A\}} F_{z}$.

Using a similar approach, the xy planar case, ${ }^{\{A\}} F_{z}=0$ and $c=0$, is presented:

$$
\Phi=\tan ^{-1}\left(\frac{c}{b}\right)=\tan ^{-1}\left(\frac{0}{b}\right)=0
$$

with ${ }^{\{A\}} F_{z}=0$ and $\sin (\Phi)=0$ equation (86) reduces to

$$
{ }^{\{D\}} F_{z}=0
$$

Replacing ${ }^{\{D\}} F_{z}$ into equation (74), we obtain

$$
\frac{{ }^{\{C\}} I_{x x} G}{(1-\gamma) L} \Upsilon=0
$$


it is required that $\frac{{ }^{\{C\}} I_{x x} G}{(1-\gamma) L} \neq 0$, thus

$$
r=0
$$

substitution on equation (58) yields,

$$
\begin{gathered}
\Phi=\Upsilon+\Gamma \\
\Gamma=0
\end{gathered}
$$

Again, equation (76) simplifies to:

$$
\left(\gamma L^{\{D\}} F_{y}-\gamma K_{\Theta} \frac{E}{L}{ }^{\{A\}} I_{z z} \Theta\right)=0
$$

which is similar to standard planar PRBMs. The previous results show that the beam will not experience twist. In addition, the angles $\Phi$ and $\Gamma$ will vanish which is the case in a planar PRBM.

For the axisymmetric beam case where ${ }^{\{C\}} I_{y y}={ }^{\{C\}} I_{z z}$, thus equation (63) yields $I_{R}=0$. Partial differentiation with respect to $\Gamma$ of equation (61) yields

$$
-\frac{d V_{B}}{d \Gamma} \delta \Gamma=-\gamma K_{\Theta} \frac{E}{L} I_{R} \Theta^{2} \sin (2 \Gamma) \delta \Gamma
$$

substitution of equations (99) into (65)

$$
\delta \Gamma:\left(\gamma L^{\{D\}} F_{z} \sin \Theta-\gamma K_{\Theta} \frac{E}{L} I_{R} \Theta^{2} \sin (2 \Gamma)\right)=0
$$


substitution of $I_{R}=0$ in equation (100) gives,

$$
\gamma L^{\{D\}} F_{z} \sin \Theta=0
$$

it is required that $\gamma L \sin \Theta \neq 0$, thus

$$
{ }^{\{D\}} F_{z}=0
$$

Equation (74) reduces to

$$
\frac{{ }^{\{C\}} I_{x x} G}{L} \Upsilon=0
$$

it is required that $\frac{{ }^{\{C\}} I_{x x} G}{L} \neq 0$, thus

$$
r=0
$$

substitution on equation (58) yields,

$$
\begin{gathered}
\Phi=\Upsilon+\Gamma \\
\Phi=\Gamma
\end{gathered}
$$

substitution of $I_{R}$ in equation (76) gives,

$$
\left(\gamma L^{\{D\}} F_{y}-\gamma K_{\Theta} \frac{E}{L}^{\{C\}} \bar{I} \Theta\right)=0
$$


The previous results show that the beam will not experience twist. Results suggest that rotation angles $\Phi$ and $\Gamma$ will be equal to $90^{\circ}-\eta$ (i.e. the bend axis is perpendicular to the force direction).

\subsection{Validation Using Finite Element Analysis}

Ansys, commercially available FEA software, was used to validate the results of the numerical integration. In the following examples, the results from the numerical integration and Ansys are presented. The beam is $1 \mathrm{~m}$ long, $0.02 \mathrm{~m}$ wide, $0.01 \mathrm{~m}$ thick, and Young's modulus is $200 \mathrm{GPa}$. In the first case, the beam is loaded under the following force parameters.

- $\mathrm{F}=600 \mathrm{~N}$ and $\eta=135^{\circ}, \xi=90^{\circ}$

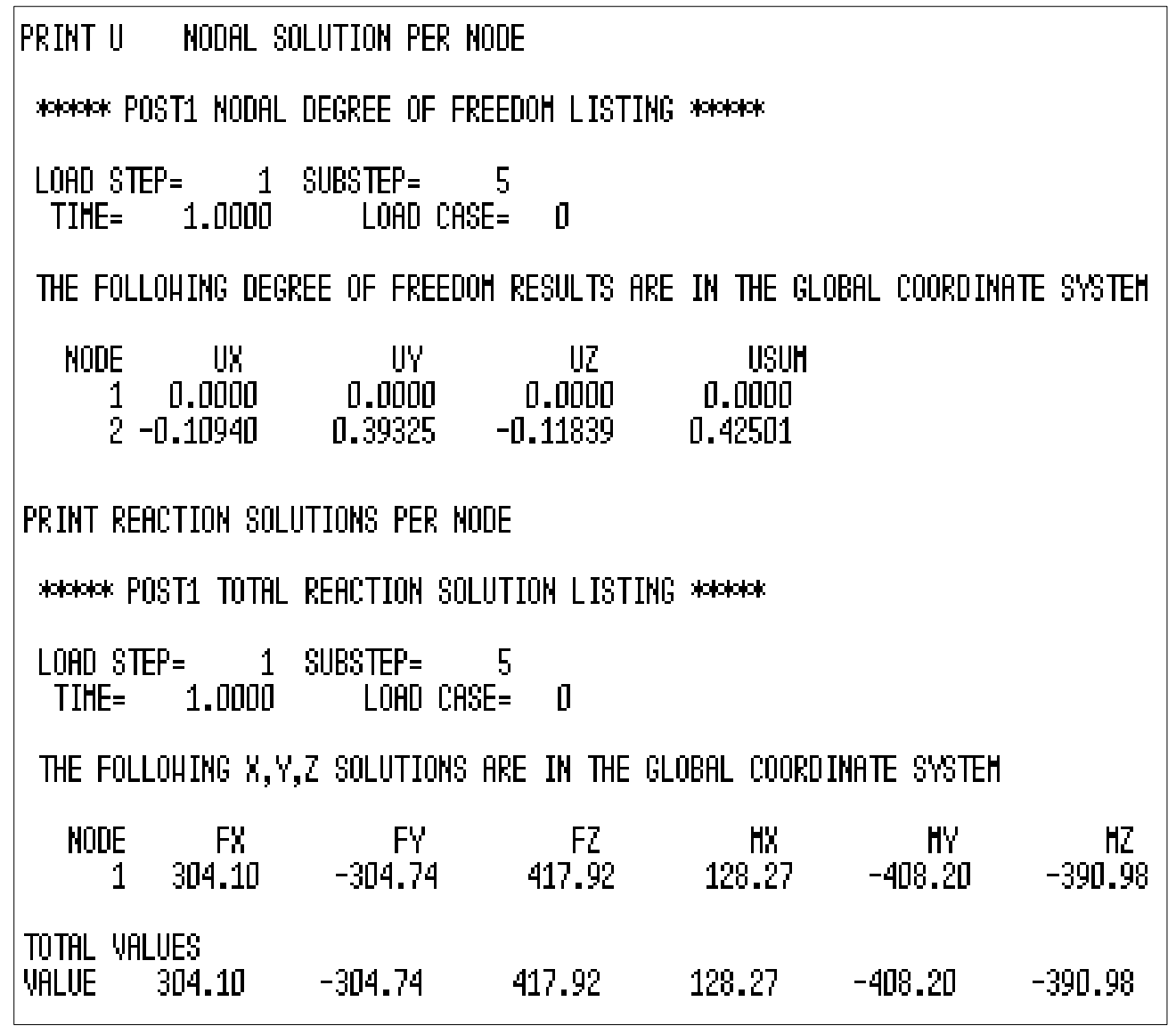

Figure 21 Ansys output for cantilever loaded with $\mathrm{F}=600 \mathrm{~N}$ 

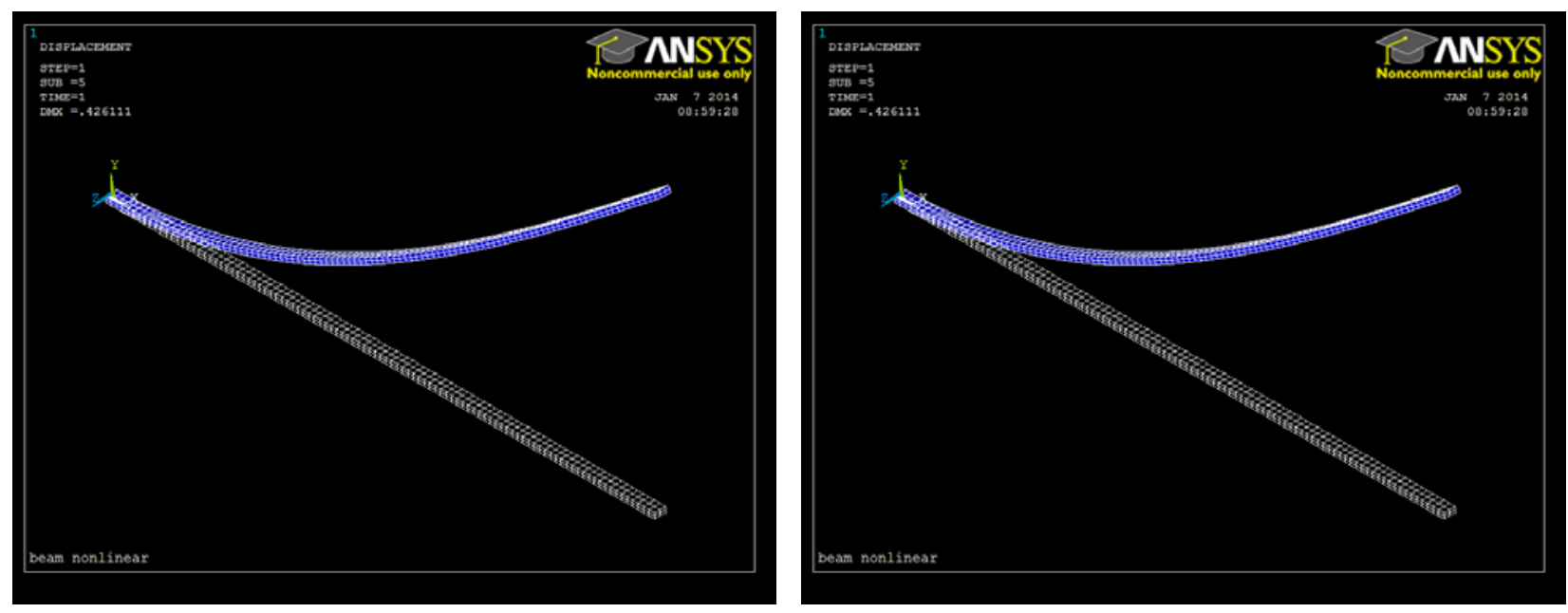

Figure 22 Deformed and undeformed beam loaded with 600N

The results summary in Figure 21and the deformed beam in Figure 22 present the deflections and reaction moments on the cantilever beam. The relative coordinate position of beam ends with respect of the fixed end was calculated by Ansys for the deformed beam. The deflections are $0.8906 \mathrm{~m}, 0.39325 \mathrm{~m}$, and $-0.11839 \mathrm{~m}$ for $\mathrm{a}, \mathrm{b}$ and c respectively. The moment reactions at the fixed end of the beam in the $\mathrm{x}$-, y- and z-components were $128.27 \mathrm{Nm},-408.20 \mathrm{Nm}$ and $-390.98 \mathrm{Nm}$ respectively.

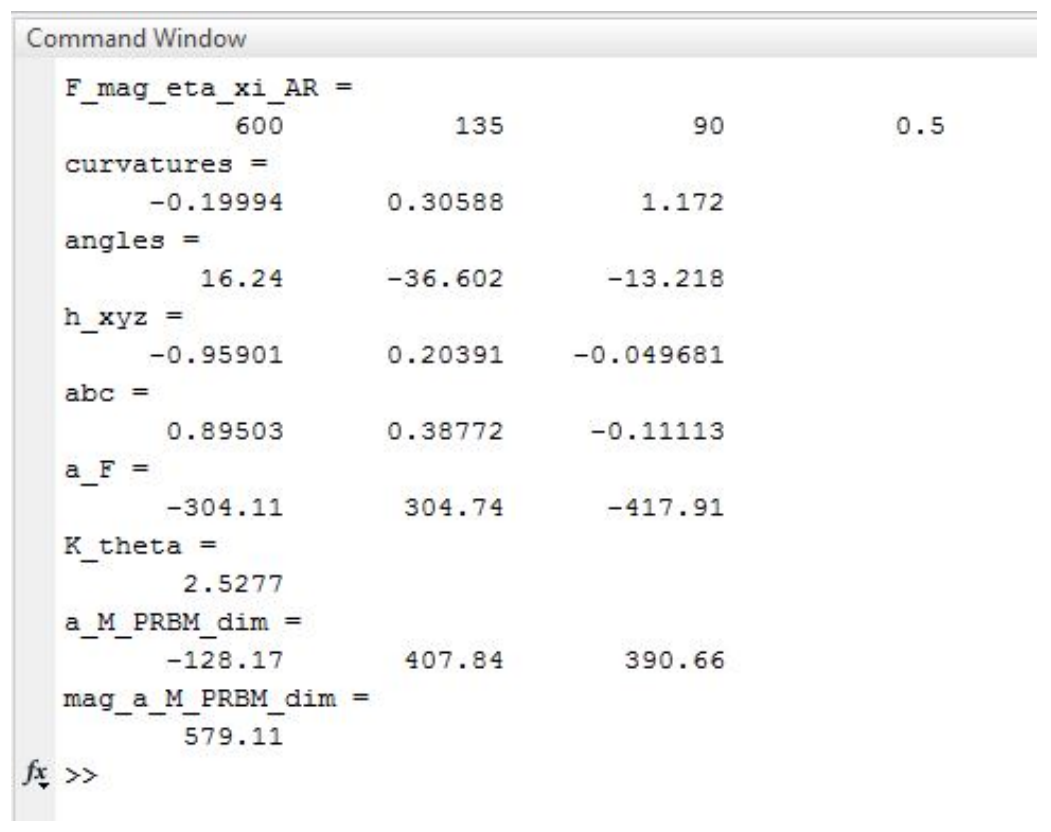

Figure 23 Matlab output for cantilever loaded with F=600N 
Results from the numerical integration are presented in Figure 23. The deflections are $0.8905 \mathrm{~m}$, $0.38772 \mathrm{~m}$, and $-0.1111 \mathrm{~m}$ for $\mathrm{a}, \mathrm{b}$ and c respectively The equivalent applied moments at the fixed end of the beam in the $\mathrm{x}-$, $\mathrm{y}$ - and z-components were $128.17 \mathrm{Nm},-407.84 \mathrm{Nm}$ and $390.66 \mathrm{Nm}$ respectively.

In the second case, the beam is loaded under the following force parameters:

- $F=750 \mathrm{~N}$ and $\eta=120^{\circ}, \xi=60^{\circ}$

The results summary in Figure 24 and the deformed beam in Figure 25 present the deflections and reaction moments on the cantilever beam. The relative coordinate position of beam ends with respect of the fixed end was calculated by Ansys for the deformed beam. The deflections are $0.79176 \mathrm{~m}, 0.53751 \mathrm{~m}$, and $-0.1162 \mathrm{~m}$ for a, b and c respectively. The moment reactions at the fixed end of the beam in the $\mathrm{x}-, \mathrm{y}-$ and z-components were $159.77 \mathrm{Nm},-362.87 \mathrm{Nm}$ and -589.59 Nm respectively.

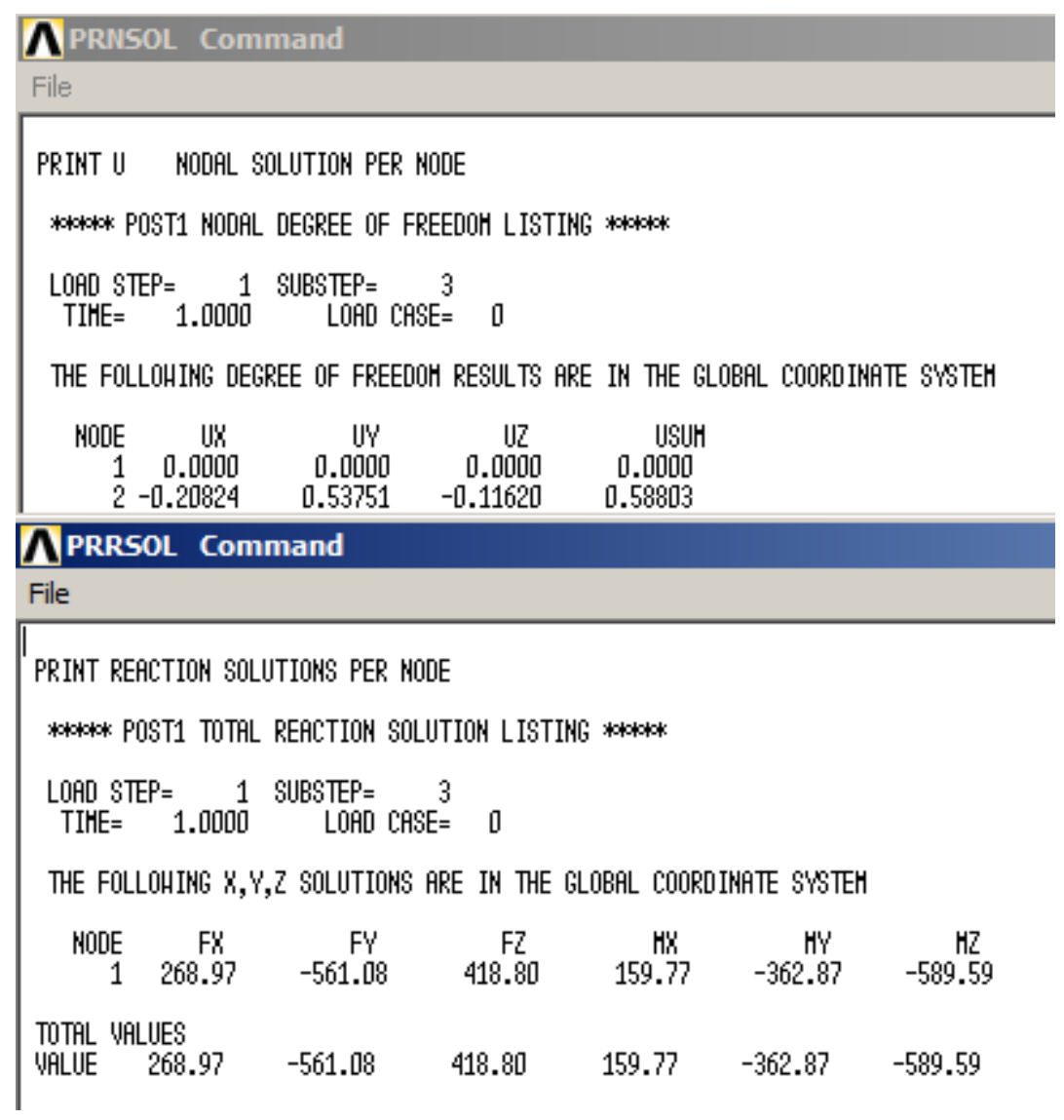

Figure 24 Ansys output for cantilever loaded with $F=750 \mathrm{~N}$ 


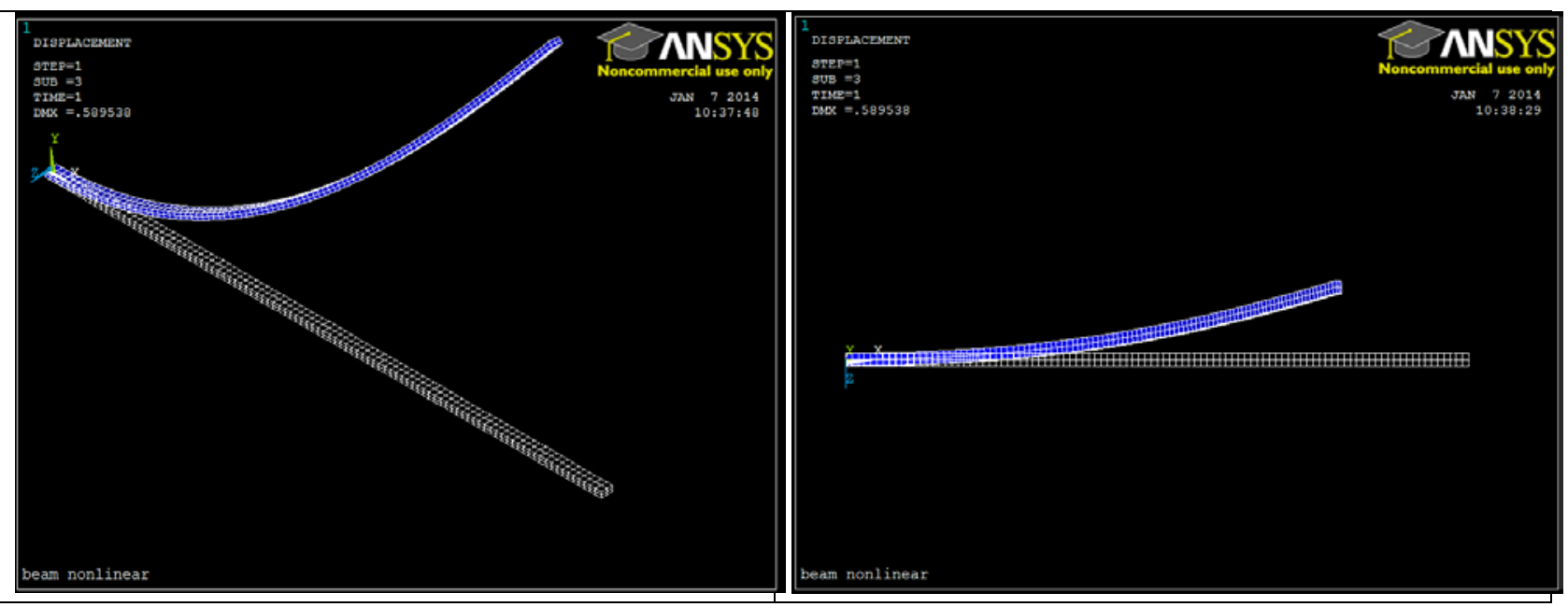

Figure 25 Deformed and undeformed beam loaded with 750N

Results from the numerical integration are presented in Figure 26. The deflections are 0.0.79651 m, $0.53471 \mathrm{~m}$, and $-0.10524 \mathrm{~m}$ for a, b and c respectively The equivalent applied moments at the fixed end of the beam in the $\mathrm{x}-$, $\mathrm{y}$ - and z-components were $-164.86 \mathrm{Nm}, 361.84 \mathrm{Nm}$ and $590.73 \mathrm{Nm}$ respectively.

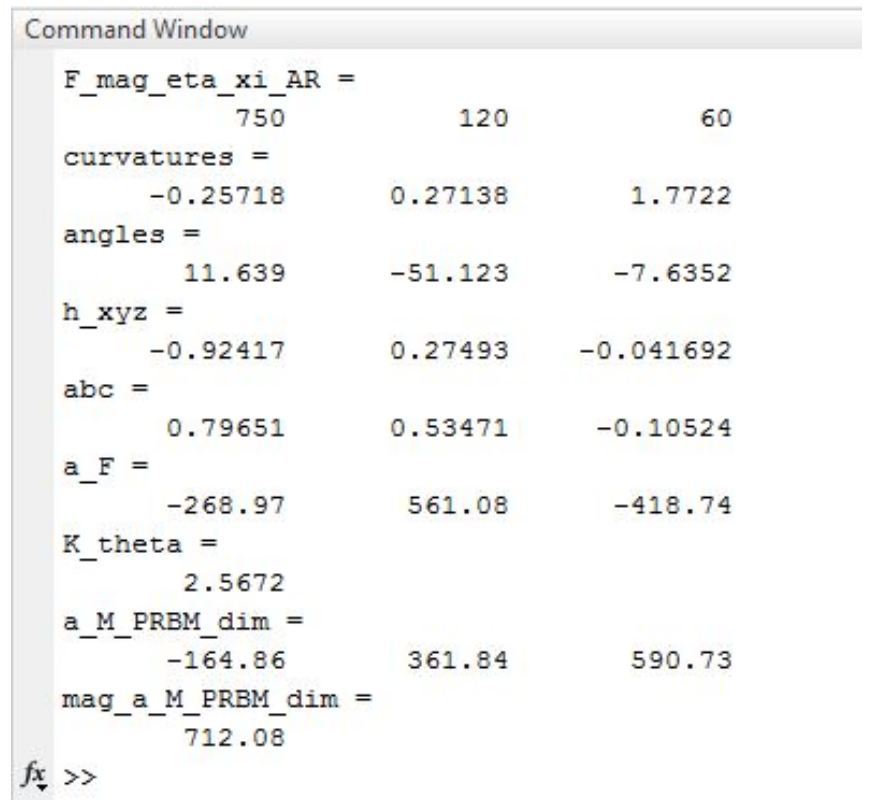

Figure 26 Matlab output for cantilever loaded with $F=750 \mathrm{~N}$ 
The previous results demonstrate the agreement between FEA and numerical integration in terms of position of the free end and the Moments and force reactions at the fixed end of the beam. The small relative error in some of the position coordinates is due to the inextensibility of the beam in the differential equations whereas in FEA the effective length of the beam can change depending on the loading conditions. 


\section{CHAPTER 5: NON-DIMENSIONAL NUMERICAL INTEGRATION}

This chapter presents a non-dimensionalized form of the governing equations presented in the Chapter 3. Solving the equations in non-dimensional form includes a broader range of materials, cross sectional aspect ratios and force combinations that represent infinite loading conditions in the same integration trajectory.

\subsection{Characteristic Length Scale}

The system of differential equations was non-dimensionalized with a characteristic length scale, $\lambda$, similar to one used by Lusk given in Chapter 2 equation (24)[30].

$$
\lambda=\sqrt{\frac{E^{\{A\}} I_{z z}}{\|F\| A R}}
$$

where, $E$ is Young's modulus. ${ }^{\{\mathrm{A}\}} I_{\mathrm{zz}}$ is the second moment of area with respect the z-axis at the fixed end of the beam. $\|F\|$ is the force magnitude applied at the free-end, and $A R$ is aspect ratio of the rectangular cross-sectional area defined as width, $w$, divided by height, $h$. Figure 27 illustrates the orientation of the cross sectional area with respect of the coordinate system $\{A\}$. Non-dimensional forms of the model parameters are defined in Table 2.

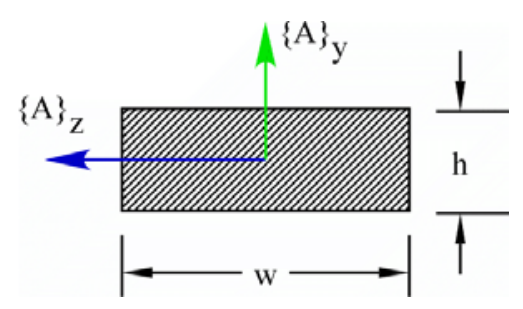

Figure 27 Rectangular cross section at fixed end 
Table 2 Non-dimensional form of parameters

\begin{tabular}{|l|c|c|}
\hline & Dimensional & $\begin{array}{c}\text { Non-dimensional } \\
\text { example }\end{array}$ \\
\hline Curvatures & $\kappa, \tau$ & $\tilde{\kappa}=\kappa \lambda$ \\
\hline Length & $l, s, x, y, z$ & $\tilde{s}=S / \lambda$ \\
\hline Force & $\vec{F}$ & $\hat{F}=\vec{F} /\|F\|$ \\
\hline
\end{tabular}

\subsection{Nondimensional Governing Equations}

Equations (32)-(34), (38)-(40) and (35)-(37) were multiplied by $\lambda$ and $\lambda^{2}$ respectively obtaining the following system non-dimensional differential equations:

$$
\begin{aligned}
& { }^{\{H\}} \frac{d \phi}{d \tilde{s}}={ }^{\{A\}} \tilde{\tau}_{x}+{ }^{\{A\}} \tilde{\kappa}_{y}\left(\frac{\cos \phi \cos \theta}{\sin \theta}\right)-{ }^{\{A\}} \tilde{\kappa}_{z}\left(\frac{\sin \phi \cos \theta}{\sin \theta}\right) \\
& { }^{\{H\}} \frac{d \theta}{d \tilde{s}}={ }^{\{A\}} \tilde{\kappa}_{y} \sin \phi+{ }^{\{A\}} \tilde{\kappa}_{z} \cos \phi \\
& \frac{{ }^{\{H\}}}{d \psi}=\frac{-{ }^{\{A\}} \tilde{\kappa}_{y} \cos \phi+{ }^{\{A\}} \tilde{\kappa}_{z} \sin \phi}{\sin \theta} \\
& { }^{\{A\}} \frac{d \tilde{\tau}_{x}}{d \tilde{s}}=\left(\frac{2(1+v)\left(1-A R^{2}\right)}{1+A R^{2}}\right)\{A\} \tilde{\kappa}_{y}{ }^{\{A\}} \tilde{\kappa}_{z} \\
& { }^{\{A\}} \frac{d \tilde{\kappa}_{y}}{d \tilde{s}}=\left(A R^{2}-\frac{\left(1+A R^{2}\right)}{2(1+v)}\right){ }^{\{A\}} \tilde{\tau}_{x}{ }^{\{A\}} \tilde{\kappa}_{z}+A R^{\{A\}} \hat{F}_{z} \\
& { }^{\{A\}} \frac{d \tilde{\kappa}_{z}}{d \tilde{s}}=\left(\frac{\left(A R^{2}-2 v-1\right)}{2(1+v) A R^{2}}\right){ }^{\{A\}} \tilde{\tau}_{x}{ }^{\{A\}} \tilde{\kappa}_{y}-\frac{\{A\}}{A R} \\
& { }^{\{H\}} \frac{d \tilde{x}}{d \tilde{s}}=\cos \theta \\
& { }^{\{H\}} \frac{d \tilde{y}}{d \tilde{s}}=\cos \psi \sin \theta \\
& { }^{\{H\}} \frac{d \tilde{z}}{d \tilde{s}}=\sin \psi \sin \theta
\end{aligned}
$$


where $v$ is Poisson's ratio. The ODE45 command in Matlab was used to numerically integrate the system of differential equations. These differential equations are solved with initial conditions of $\tilde{s}=0$ at the free-end of the cantilever beam, assuming no torques and no displacements or rotations in the fixed end $\left(\tilde{\tau}_{x}=\tilde{\kappa}_{y}=\phi=\theta=\psi=\tilde{x}=\tilde{y}=\tilde{z}=0\right.$ ). The numerical integration stops when a maximum in the bending curvature is found. Equations (41) and (42) are used at every integration step to transform $\hat{F}$ and position vector $(\tilde{x}, \tilde{y}$ and $\tilde{z})$ from frame $\{\mathrm{H}\}$ to $\{\mathrm{A}\}$ respectively.

Table 3 shows the typical structure of the results from numerical integration of non-dimensional form of equations. In order to navigate through results three inputs are required. These inputs are direction of the non-follower force, $\eta$ and $\xi$, aspect ratio (height/width), and the equivalent non-dimensional length (e.g. $\tilde{s}=\frac{l}{\sqrt{\frac{E I_{z Z}}{\|F\| A R}}}$ ). To provide an example, imagine a small scale beam of $100 \mathrm{~mm}$ of length, $1 \mathrm{~mm}$ of height, $5 \mathrm{~mm}$ of width, loaded at the free end with a force of $10.25 \mathrm{~N}$ with a follower force of angles $\eta$ and $\xi$ of $120^{\circ}$ and $65^{\circ}$ respectively. The nondimensional parameter $\tilde{s}$ is computed:

$$
\tilde{s}=\frac{l}{\sqrt{\frac{E I}{\|F\| A R}}}=\frac{0.1 \mathrm{~m}}{\sqrt{\frac{200 G p a\left(\frac{0.005 * 0.001^{3}}{12}\right) m^{4}}{10.25 N * \frac{0.001}{0.005}}}}=0.496
$$

Results suggest that the beam will deflect $32 \%$ of its length in the positive y-axis, $9 \%$ in the negative z-axis with respect of the $\{\mathrm{A}\}$ frame. Pseudo-rigid body angles $\Psi, \Theta$ and $\Phi$ equal to $1.463^{\circ}$, $22.79^{\circ}$, and $1.54^{\circ}$ respectively, and a characteristic radius factor of 0.834 . 
Table 3 Typical results from numerical integration

\begin{tabular}{|c|c|c|c|c|c|c|c|c|c|c|c|c|c|c|c|c|c|c|c|c|}
\hline & & & & & & & & & & & & & & & & $\Gamma 2$ & 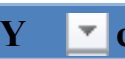 & $c_{\psi}=$ & $c_{\Theta}$ & 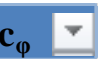 \\
\hline 20 & 60 & 024 & 2 & 20.0 & 61.1 & & 013 & 0.000 & 1.527 & 0.834 & 0.902 & -0.003 & 0.226 & -1.524 & 2.501 & -1.524 & 0.000 & 0.999 & 1.250 & 1.000 \\
\hline 0 & 0 & & & 0.1 & 46 & 98 & & & 26 & 334 & 32 & & 06 & 18 & & & & 992 & 249 & 002 \\
\hline 120 & 0 & & & 0.1 & 70.4 & & & & .524 & .835 & & & & & & & & 976 & 248 & .006 \\
\hline 20 & 0 & & & 0.3 & 78.7 & 2 & 4 & & 525 & .835 & 327 & & 71 & & & & & 2 & 248 & 015 \\
\hline 120 & & & & & & & & & & & & & & & & & & & & .030 \\
\hline 120 & 50 & 6142 & 2 & 0.8 & 103.1 & 857 & 0.467 & -0.014 & 1.539 & 0.834 & 34.102 & -0.4 & 420 & -1.738 & 2.548 & 330 & -0.407 & 0.759 & 247 & .056 \\
\hline 120 & & & & & & & & & & & & & & & & & & & & 094 \\
\hline 120 & 60 & 0.8189 & 2 & 1.5 & 37.5 & 575 & 0.722 & -0.030 & 586 & 0.827 & 924 & & 269 & -2 & & & & 255 & 251 & .150 \\
\hline 120 & 60 & & & 1.8 & 57.6 & & & & 6 & & & & & & & & & 000 & & 234 \\
\hline 120 & 60 & 236 & 2 & 21.8 & 178.8 & 0.165 & 0.805 & -0.054 & 1.674 & 0.807 & 92 & -3.148 & 24.184 & 836 & 2.726 & 381 & 455 & .000 & 262 & 374 \\
\hline 120 & 65 & 0.0992 & 0.2 & 120.0 & 66.1 & 1.000 & 0.013 & 0.000 & 1.459 & 0.834 & 886 & -0.002 & 22 & -1 & & -1 & 0.000 & 0.999 & 250 & 1.000 \\
\hline 120 & 65 & 1983 & 0.2 & 120.1 & 69.5 & 0.998 & 0.052 & -0.001 & 1.458 & 0.834 & 3.561 & -0.012 & 0.888 & -1.453 & 2.502 & -1.449 & -0.005 & 0.993 & 249 & .002 \\
\hline 120 & 65 & 0.2975 & & 120.1 & 75.2 & 0.992 & 0.117 & -0.003 & 1.458 & 0.834 & 8.068 & & 2.007 & 455 & 506 & -1 & -0.0 & 0.979 & 249 & 1.005 \\
\hline 120 & 65 & 3966 & & 20.2 & 83.3 & 0.974 & 208 & -0.005 & 1.459 & 0.835 & 14.466 & & 3.590 & -1.478 & 2.512 & -1 & -0.075 & 0.948 & 248 & . 013 \\
\hline 120 & 65 & 4958 & & 120.4 & 93.8 & 0.935 & 323 & -0.009 & 1.463 & 0.834 & 93 & & 49 & & & -1 & & 887 & 248 & .027 \\
\hline 120 & 65 & 0.5950 & & 120.6 & 106.8 & 0.865 & & & 1.474 & 0.833 & & & 8.196 & 568 & & & -0.3 & 0.779 & 1.248 & 1.051 \\
\hline 120 & 65 & 0.6941 & 0.2 & 120.9 & 122.1 & 0.756 & 0.587 & -0.019 & 1.493 & 0.831 & 45.050 & -0.699 & 11.238 & -1.893 & 2.569 & -1.220 & -0.673 & 0.602 & 1.249 & 1.086 \\
\hline 120 & 65 & .7933 & & 121.2 & 139.6 & 0.605 & 0.704 & -0.028 & 1.521 & 0.826 & 58. & & 14.773 & -2.253 & 2.607 & -1.154 & -1.099 & 0.320 & .252 & 1.137 \\
\hline 120 & 65 & 0.8924 & & 121.5 & 158.7 & 0.420 & 0.782 & -0.038 & 1.561 & 0.818 & 73.066 & & 18.784 & -2.790 & 2.653 & -1.145 & -1.645 & 1.000 & .257 & .212 \\
\hline 120 & 65 & 0.9916 & 0.2 & 121.5 & 178.7 & 0.222 & 0.805 & -0.050 & 1.610 & 0.807 & 87.924 & -2.769 & 23.236 & -3.554 & 2.704 & -1.276 & -2.278 & 1.000 & 1.264 & 1.329 \\
\hline
\end{tabular}




\section{CHAPTER 6: RESULTS}

\subsection{Results from Numerical Integration}

This chapter presents the results of the numerical integration of the non-dimensional governing equations of the cantilever beam with rectangular cross section loaded with an arbitrary force and no moment at the free end for different aspect ratio. The following figures are three dimensional with $\eta$ and $\xi$ in the $\mathrm{x}$ and $\mathrm{y}$ axis respectively which represent all the possible load directions. In this dissertation, the data set has stopping criteria of $70^{\circ}$ as the maximum PRMB angle, $\Theta$ which is the limit used in the planar case by Howell [1]. Although more data was generated, the results presented are focused on this region, because of its practicality and numerical stability. In fact, fits can be found in this region with good fidelity.

In the next paragraphs, the plots and interpretation of the results for $A R=1$ which is a square cross section, and $A R=0.2$, a thin wide cross section, will be presented.

Figure 28 and Figure 45 present the nondimensional length for both aspect ratios. The values of $\tilde{s}$ are higher at the region where the force direction is nearly parallel to the beam neutral axis. In fact, the active force component will be smaller in this region compared to a perpendicular force, $\xi=90^{\circ}$, therefore a larger force is required to produce identical bending moment. When comparing the results of the equally sized squared and rectangular cross sections, it can be concluded from the plot that to produce the same bending deflection the rectangular cross section requires a smaller $\tilde{s}$. This result is expected, since the beam with the rectangular cross section is thinner. Maintaining all other parameter fixed, it needs a smaller force or beam length compared to its squared counterpart to generate the same deflection. 
In Figure 45, it can be observed the stiffening effect when the force is oriented towards the smaller side of the rectangular cross section, $\eta$ approaching $180^{\circ}$, resulting in a larger nondimensional length.

Figure 29, Figure 30, and Figure 31illustrate the relative displacement in the $x-, y-$ and z-axis respectively for $A R=1$. Figure 46, Figure 47 and Figure 48 illustrate the relative displacement in the $x$-, $\mathrm{y}$ - and z-axis respectively for $A R=0.2$. As expected the relative deflection in the z-axis is significantly smaller for the wide rectangular cross section beam.

For $A R=1$, $\Phi$ illustrated in Figure 32, the PRBM twisting angle is identical to the frame rotation angle, $\Gamma$ illustrated in Figure 39, because there is no twist of the cross section along the beam, $\Upsilon$ shown in Figure 38. In addition, the final rotation that takes place in the second spherical joint, $\Psi$ illustrated in Figure 37 , is exactly opposite to $\Phi$. This is a characteristic intrinsic of axisymmetric beams. The first rotation that takes place in the second spherical joint, $\Sigma$ illustrated in Figure 36, is almost negligible.

On the other hand, for rectangular beam the twisting effect, $\Upsilon$ shown in Figure 55, is always present producing a difference between $\Phi$ and $\Gamma$ illustrated in Figure 49 and Figure 56 respectively. The flat behavior at the top surface presented by this two parameters suggest that the beam will not experience significant out of plane deflections until the force component in that direction is sufficiently large which is controlled by $\xi$. In addition, the final rotation that takes place in the second spherical joint, $\Psi$ illustrated in Figure 54, has an opposite behavior to $\Phi$, but not entirely symmetrical. The values of $\Sigma$ shown in Figure 53 still small and becomes appreciable when the force angle $\xi$ is small.

For both cross sections, the results for $\gamma, K_{\Theta}$ and $c_{\theta}$ are quasi constant along the spectrum, and more importantly are comparable to planar values for the same parameters. These results are shown in Figure 33, Figure 40, Figure 41for $A R=1$ and in Figure 50, Figure 57, Figure 58 for $A R=0.2$.

The PRBM bending angle, $\Theta$ shown in Figure 34, has a nearly constant ratio of 0.25 with the second rotation angle in the second spherical joint, $\Omega$ illustrated in Figure 35. It can also be observed the 
previously mentioned stiffening effect when $\eta$ approaches $180^{\circ}$. The other parametric angle coefficients, $c_{\phi}$, and $c_{\psi}$, relate Euler angles to PRBM angles and are equal to unity for the square cross section. Figure 59 and Figure 60 show the steady behavior of the parametric angle coefficients $c_{\phi}$, and $c_{\psi}$ for the rectangular cross section

\subsection{Fitting of Model Parameters}

It is possible to use the method of least squares to produce a regression of the parameters that have a nearly constant behavior in a particular range of interest. Regression for $K_{\theta}, \gamma, c_{\theta}, c_{\phi}$ and $c_{\Psi / \Phi}$ were calculated as a function of $\alpha, \beta, \tilde{s}$ and AR. The coefficients for each parameter were calculated using the least squares method as indicated in equation (117).

$$
\begin{gathered}
\overrightarrow{B_{Y}}=\left([X(\alpha, \beta, \tilde{s}, A R)]^{T}[X(\alpha, \beta, \tilde{s}, A R)]\right)^{-1}[X(\alpha, \beta, \tilde{s}, A R)]^{T} Y_{\text {real }} \\
Y_{\text {prediction }}=X(\alpha, \beta, \tilde{s}, A R) \vec{B}
\end{gathered}
$$

for $K_{\theta}, \gamma, c_{\theta}, c_{\phi}$ the $X(\alpha, \beta, \tilde{s}, A R)$ is given by:

$$
\begin{aligned}
X(\alpha, \beta, \tilde{s}, A R)= & {\left[1 \cos \alpha \cos \beta \sin \alpha \sin \beta \text { AR } \tilde{s} A R \tilde{s}^{2} A R \tilde{s} \cos \alpha \cos \beta \tilde{s} \sin \alpha \sin \beta \tilde{s}(1\right.} \\
& +\cos \beta \sin \alpha)]
\end{aligned}
$$

for $c_{\Psi / \Phi}$ the $X(\alpha, \beta, \tilde{s}, A R)$ is:

$$
\begin{aligned}
X(\alpha, \beta, \tilde{s}, A R)= & {\left[1 \cos \alpha \cos \beta \sin \alpha \sin \beta A R \tilde{s} A R \tilde{s}^{2} A R \tilde{s} \cos \alpha \cos \beta \tilde{s} \sin \alpha \sin \beta\right.} \\
& \tilde{s}(1+\cos \beta \sin \alpha) \sin \beta(\cos \alpha-1)-\cos \alpha]
\end{aligned}
$$


These $\mathrm{X}$ functions were chosen by a combination of studying the results profiles and trial and error. The results for the coefficient vectors are shown in the Appendix B for different aspect ratios. In addition, results of a statistical analysis of the constants parameters are also presented in the Appendix B. 


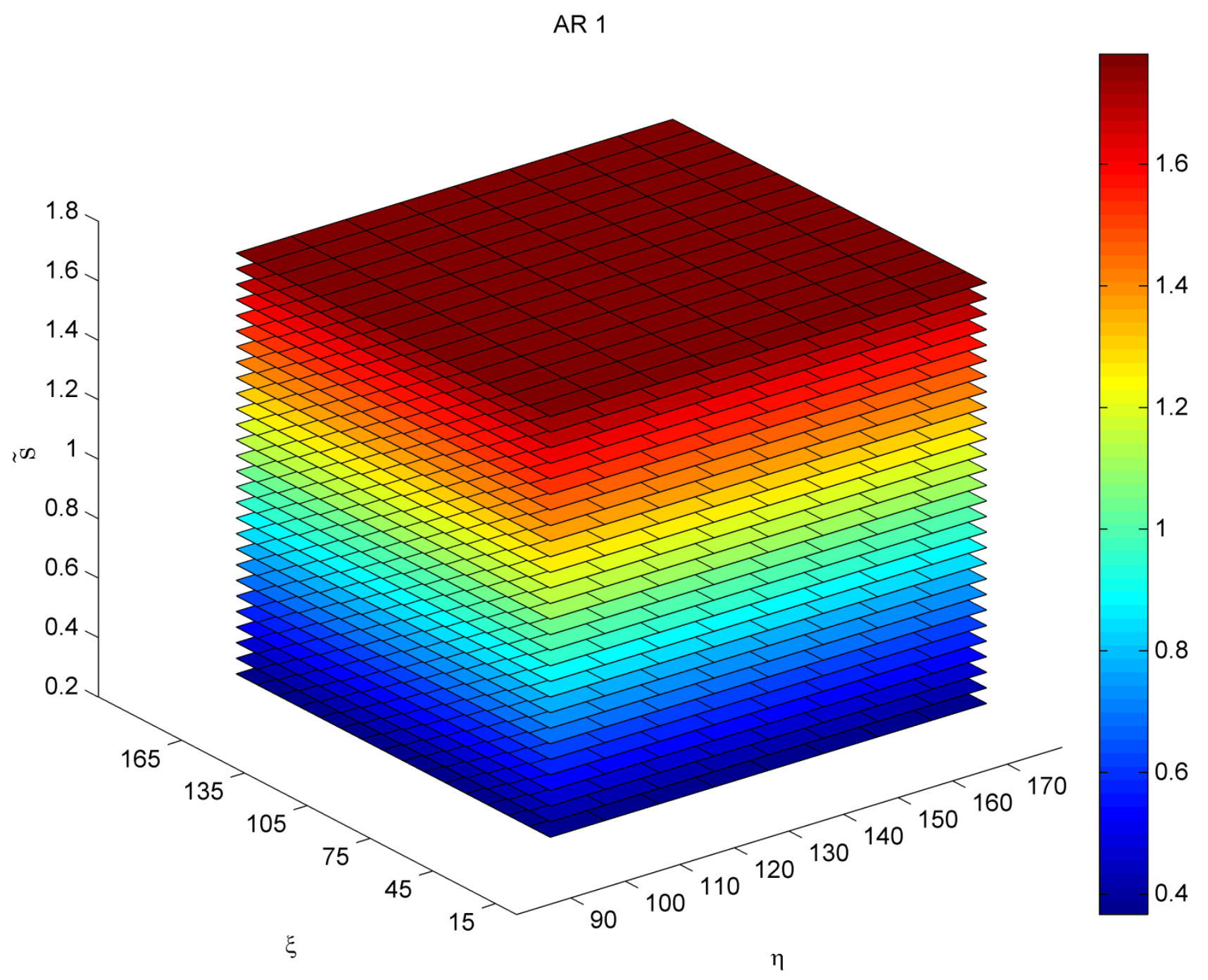

Figure 28 Nondimensional length for $A R 1$ 


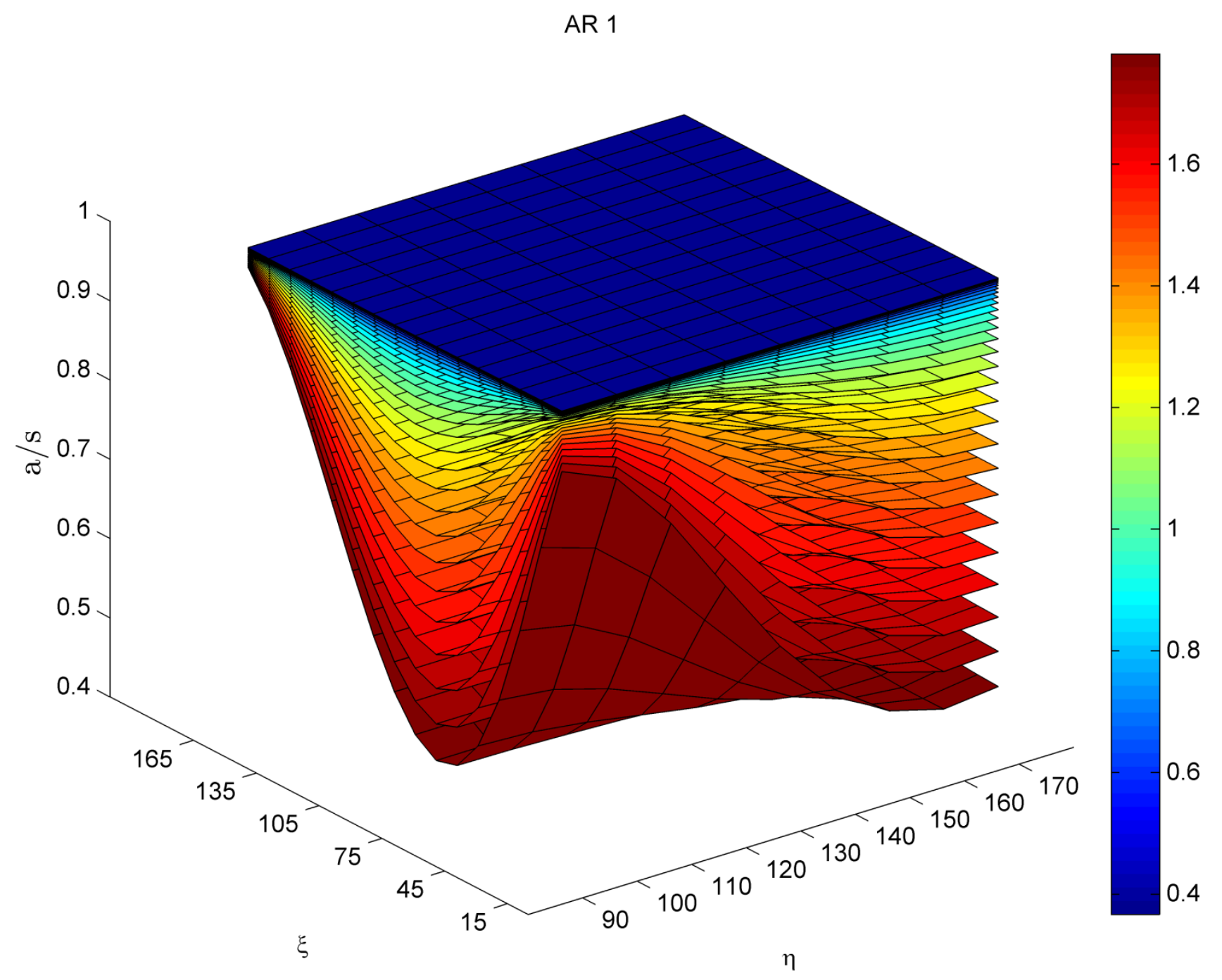

Figure $29 a / s$ for $A R 1$ 
AR 1

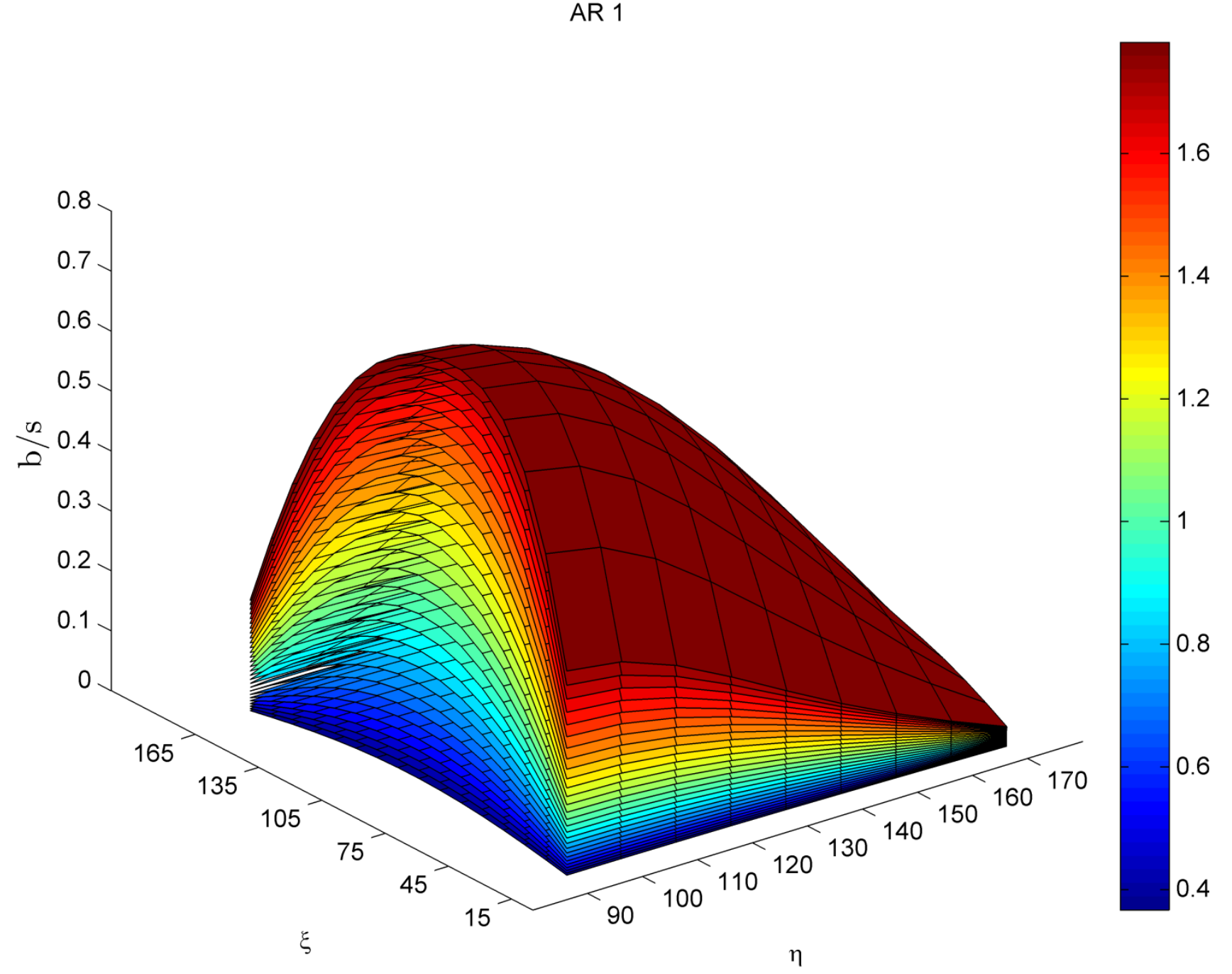

Figure $30 \mathrm{~b} / \mathrm{s}$ for $A R 1$ 


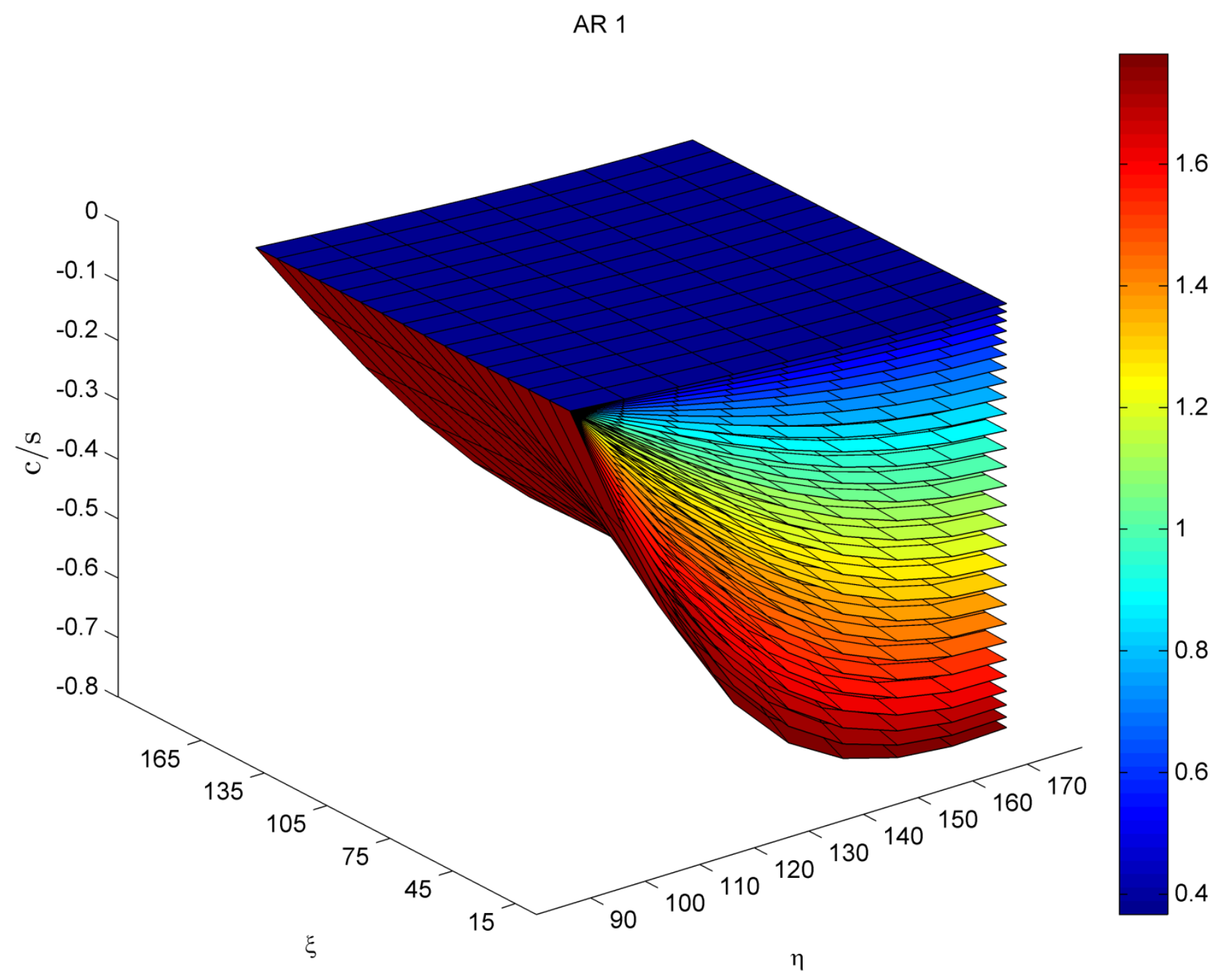

Figure $31 \mathrm{c} / \mathrm{s}$ for $A R 1$ 
AR 1

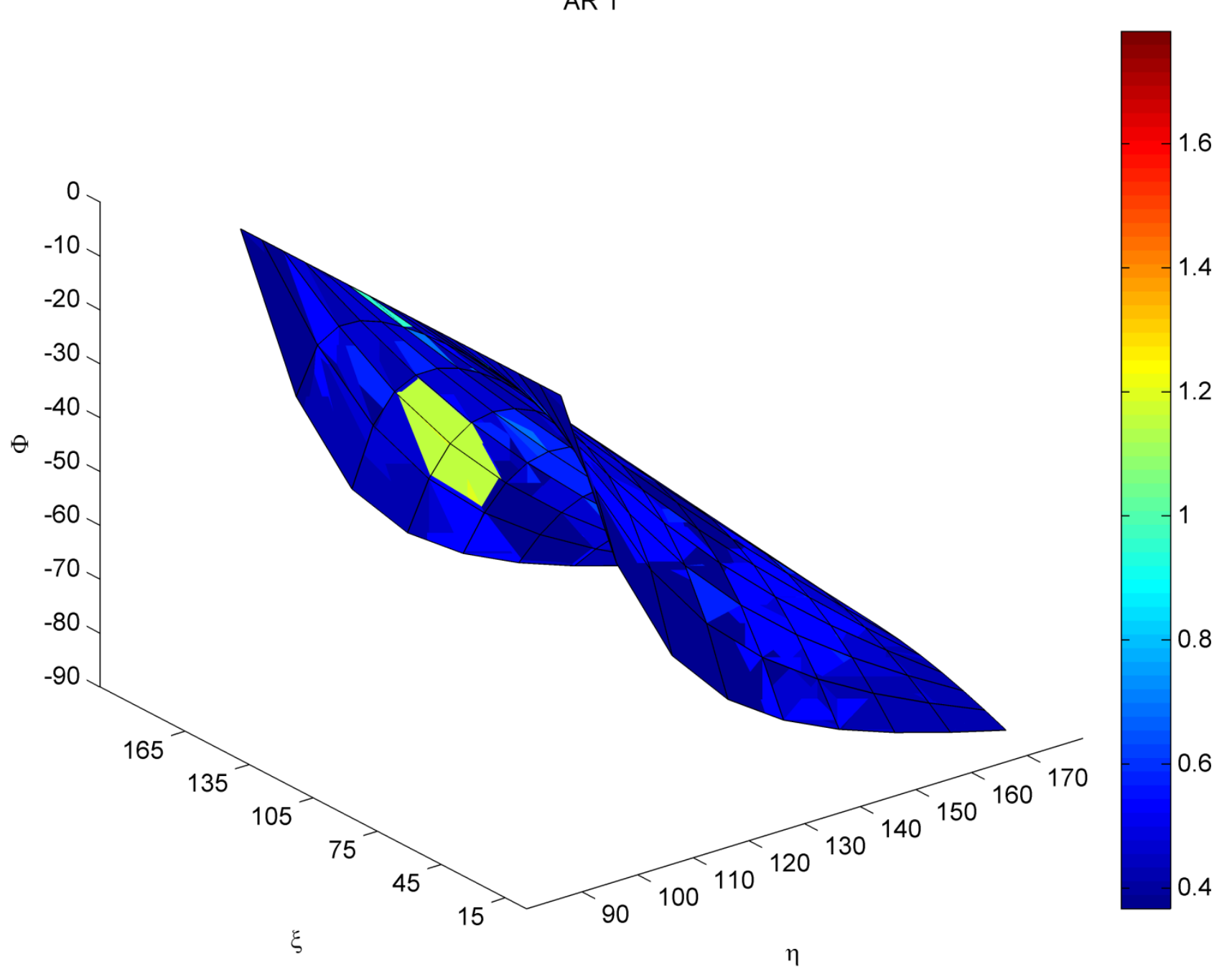

Figure $32 \Phi$ for $A R 1$ 


\section{AR 1}

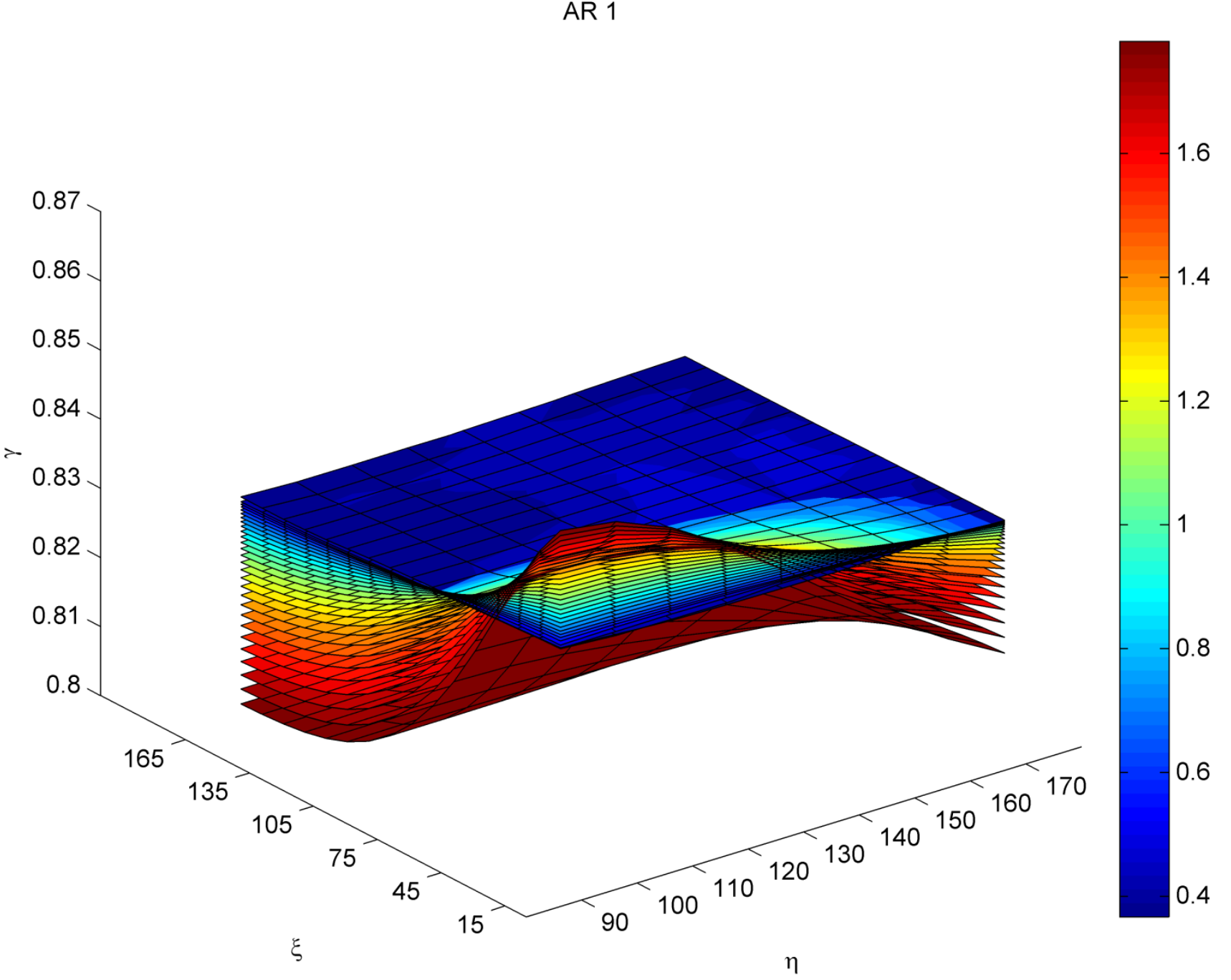

Figure $33 \gamma$ for $A R 1$ 


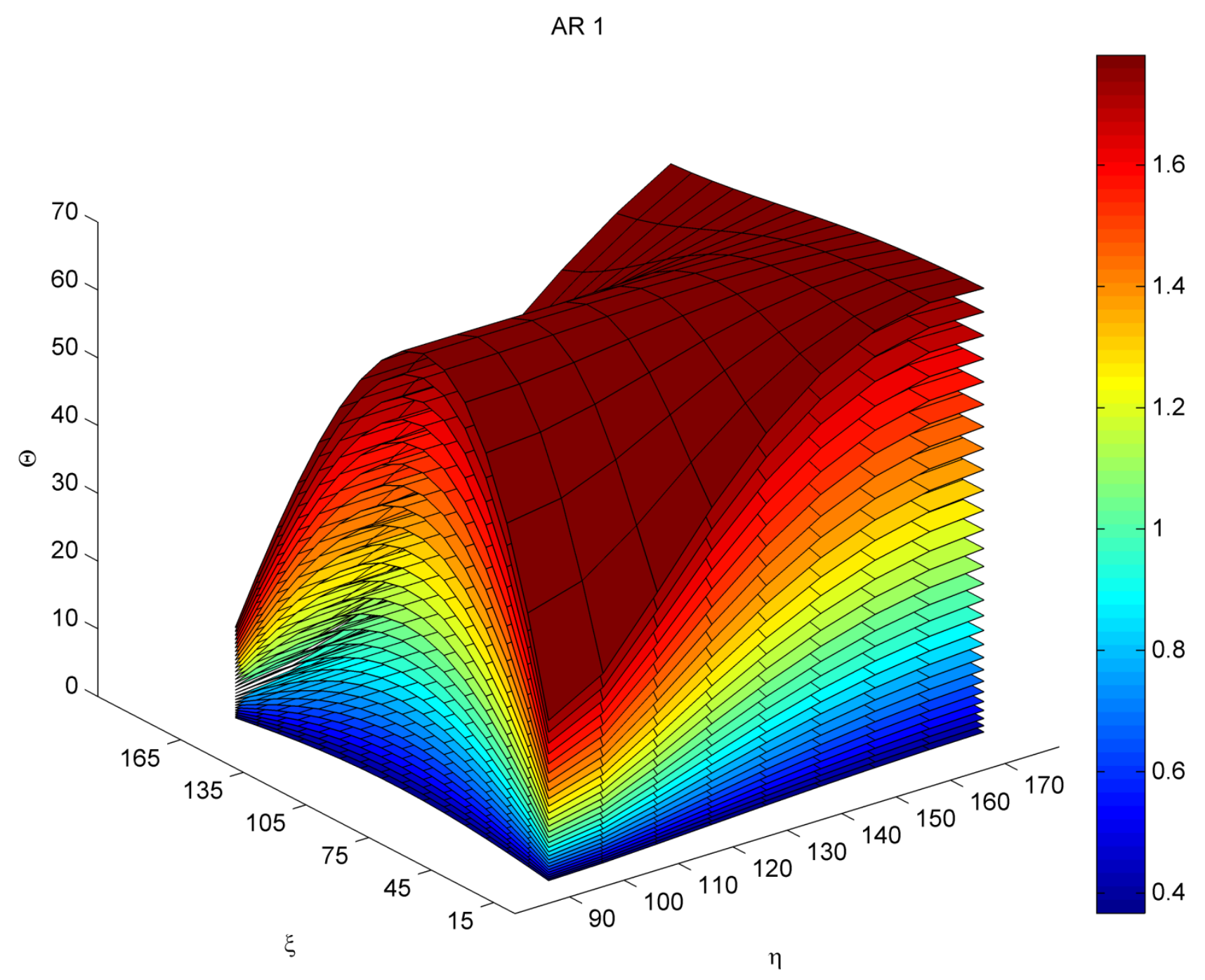

Figure $34 \Theta$ for $A R 1$ 


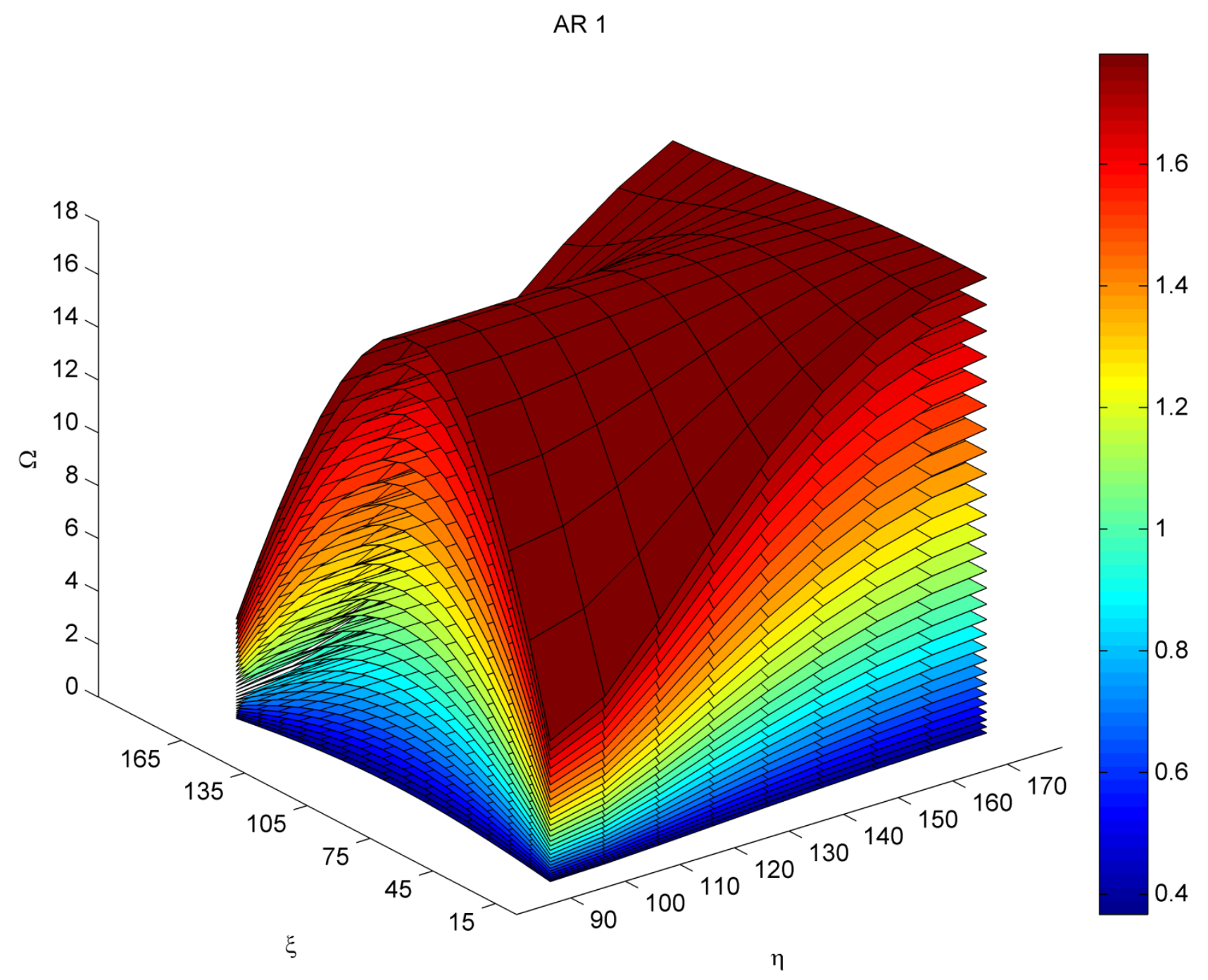

Figure $35 \Omega$ for $A R 1$ 
AR 1

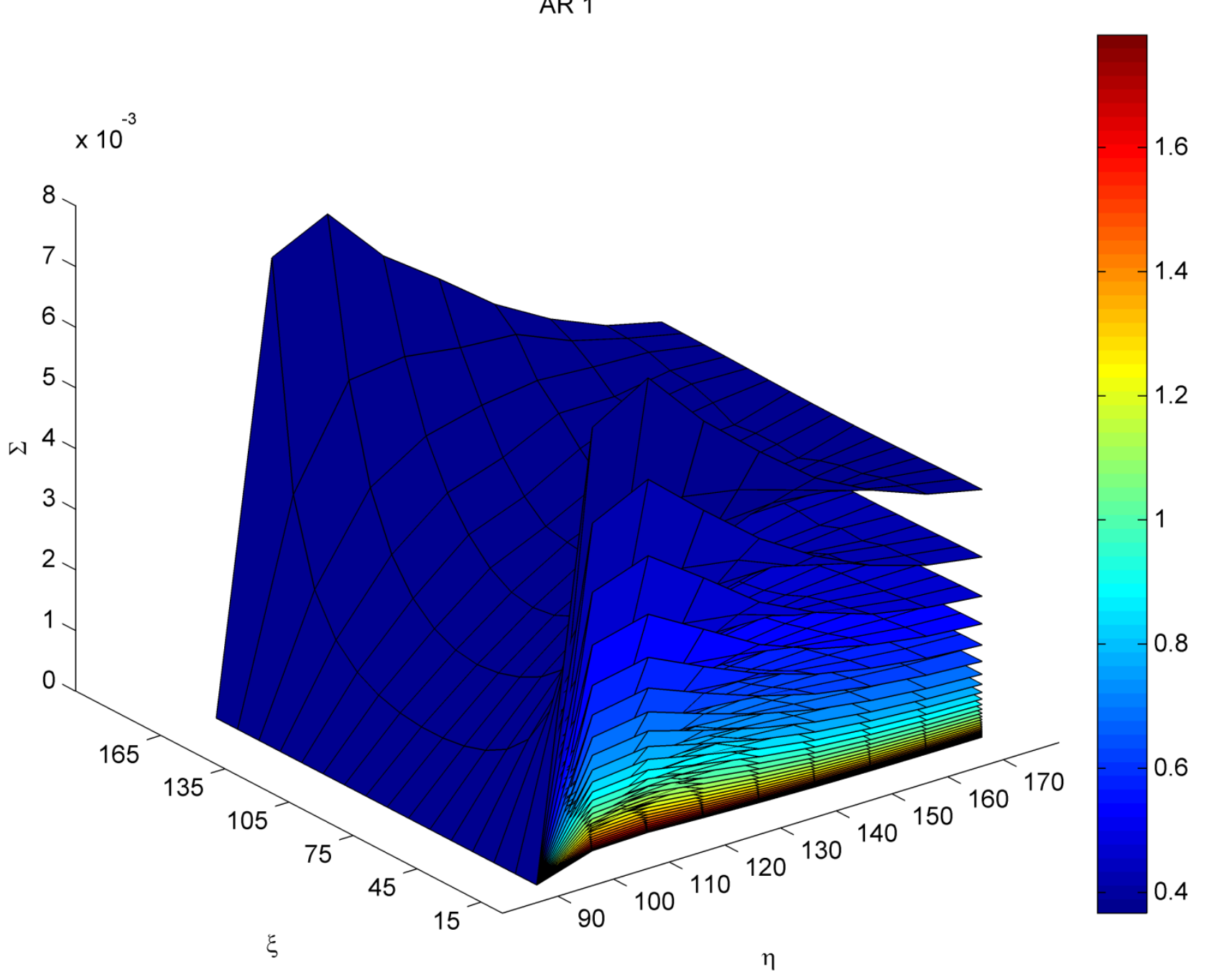

Figure $36 \Sigma$ for $A R 1$ 


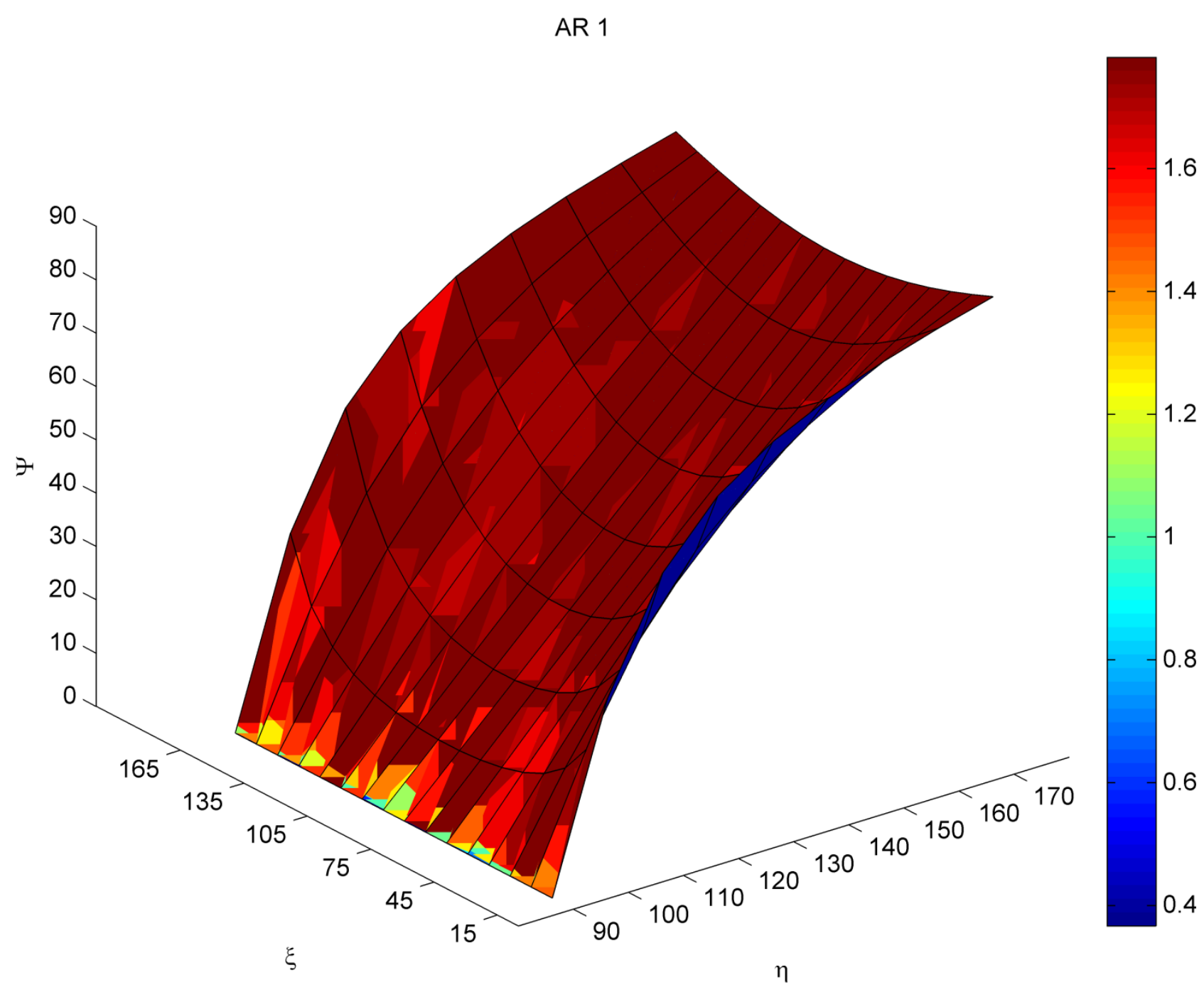

Figure $37 \Psi$ for $A R 1$ 
AR 1

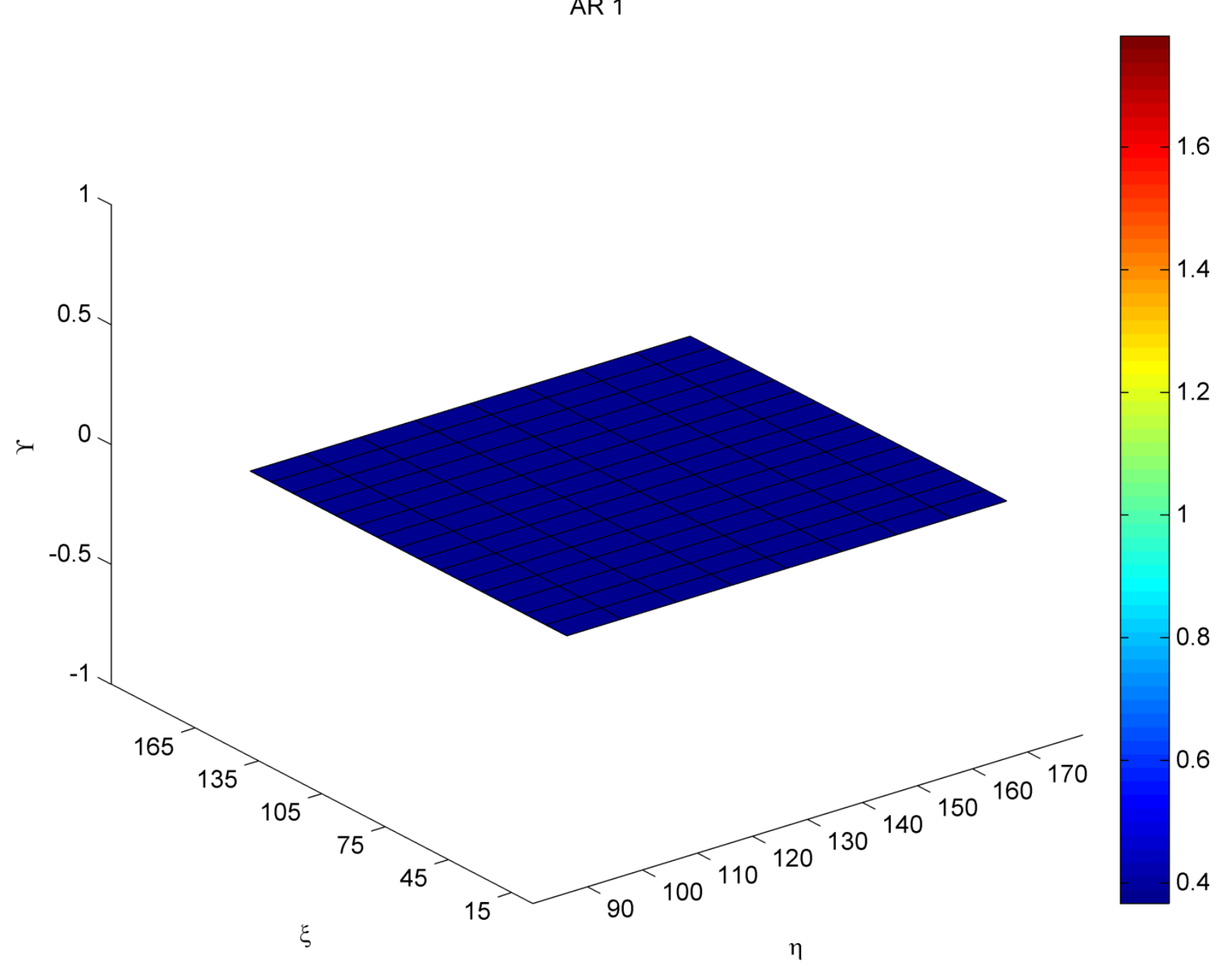

Figure $38 \Upsilon$ for $A R 1$ 
AR 1

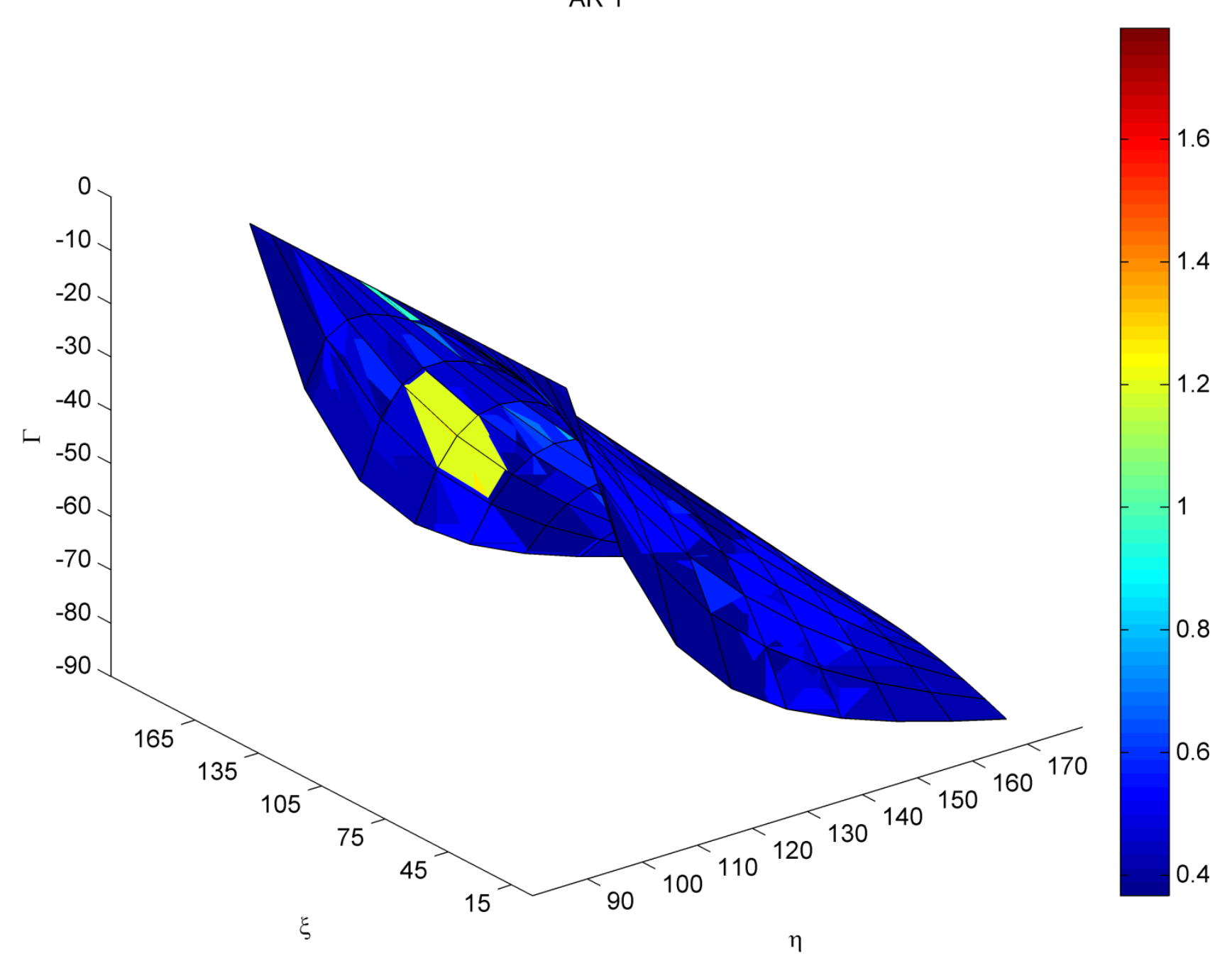

Figure $39 \Gamma$ for $A R 1$ 
AR 1

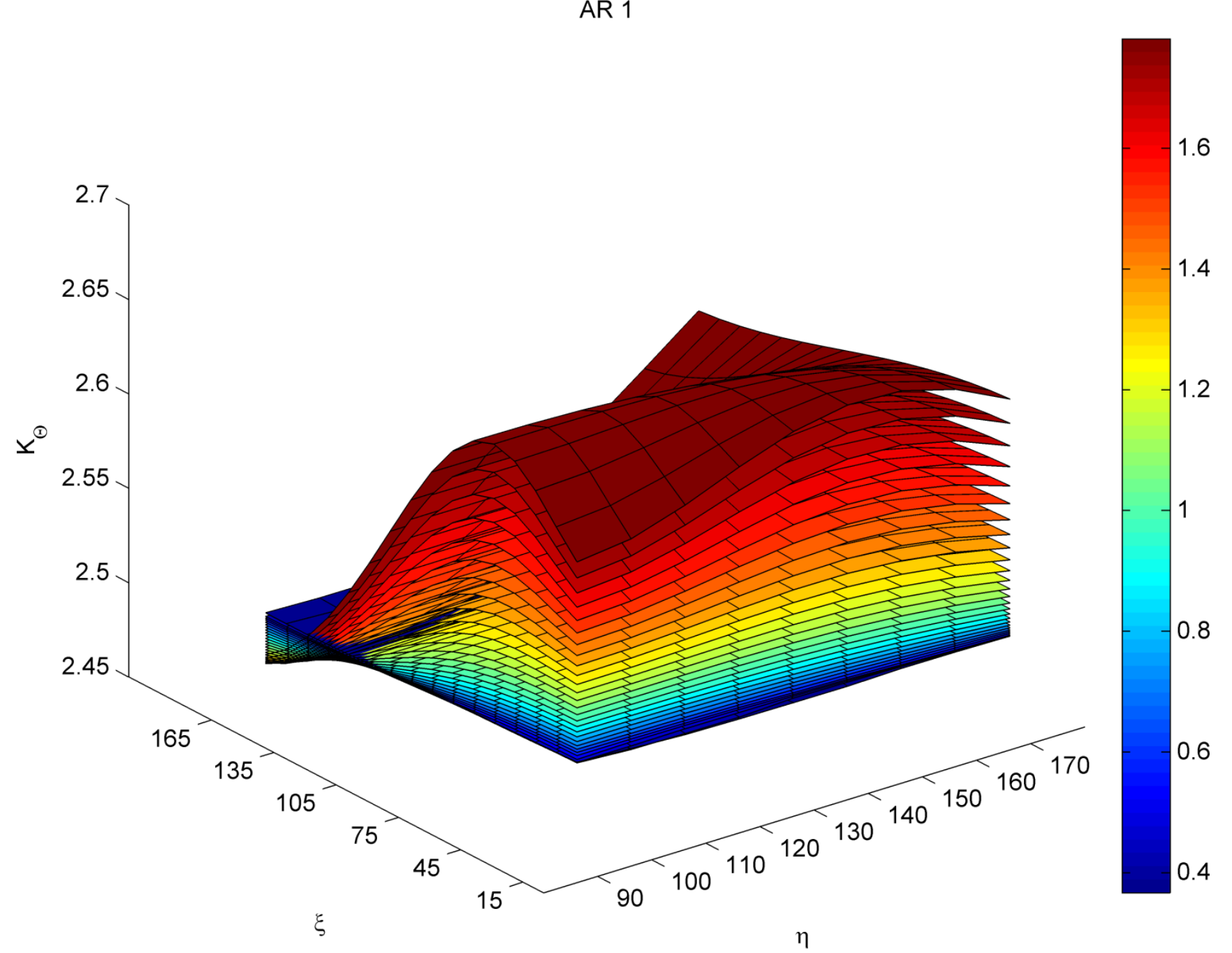

Figure $40 K_{\Theta}$ for $A R 1$ 
AR 1

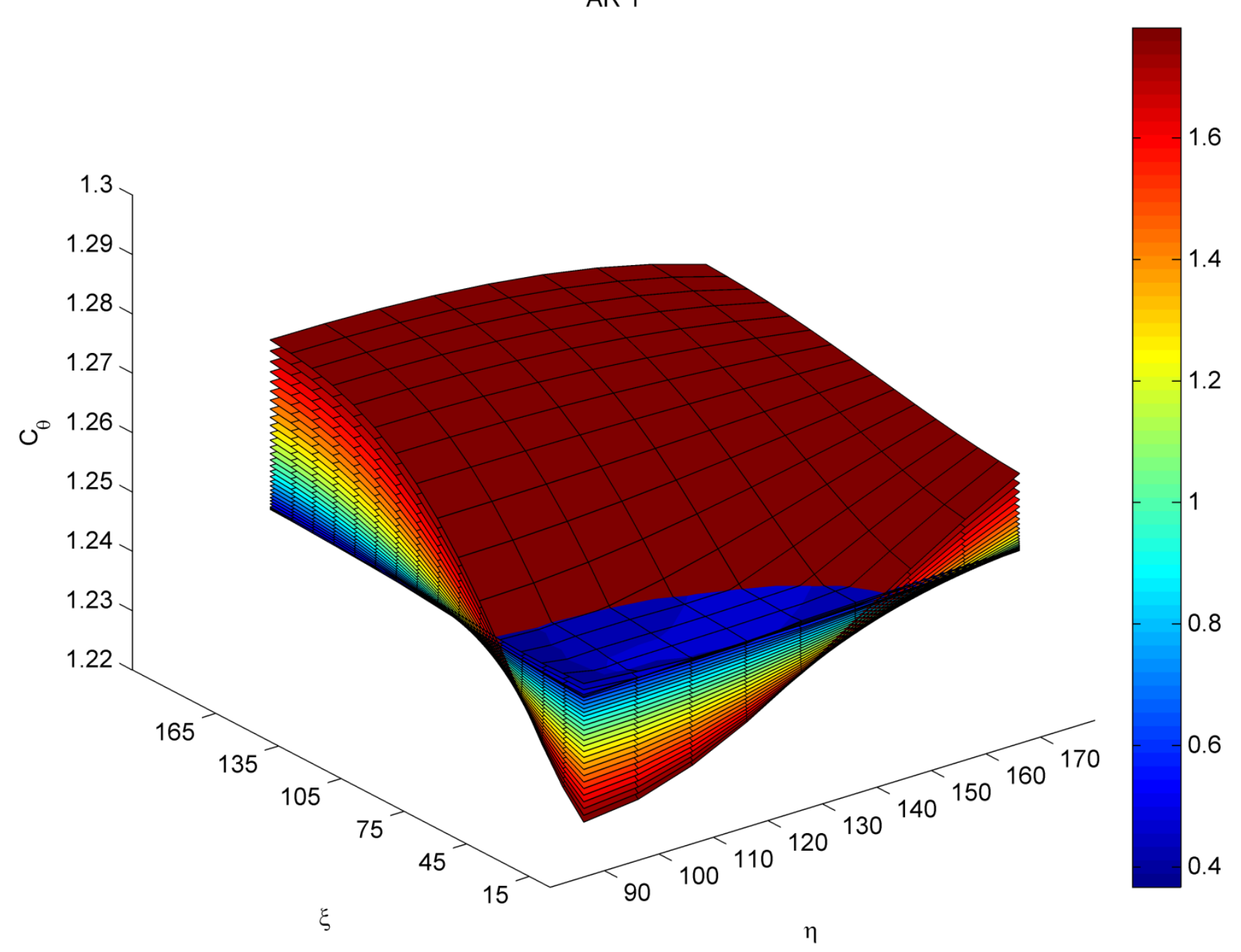

Figure $41 c_{\theta}$ for $A R 1$ 


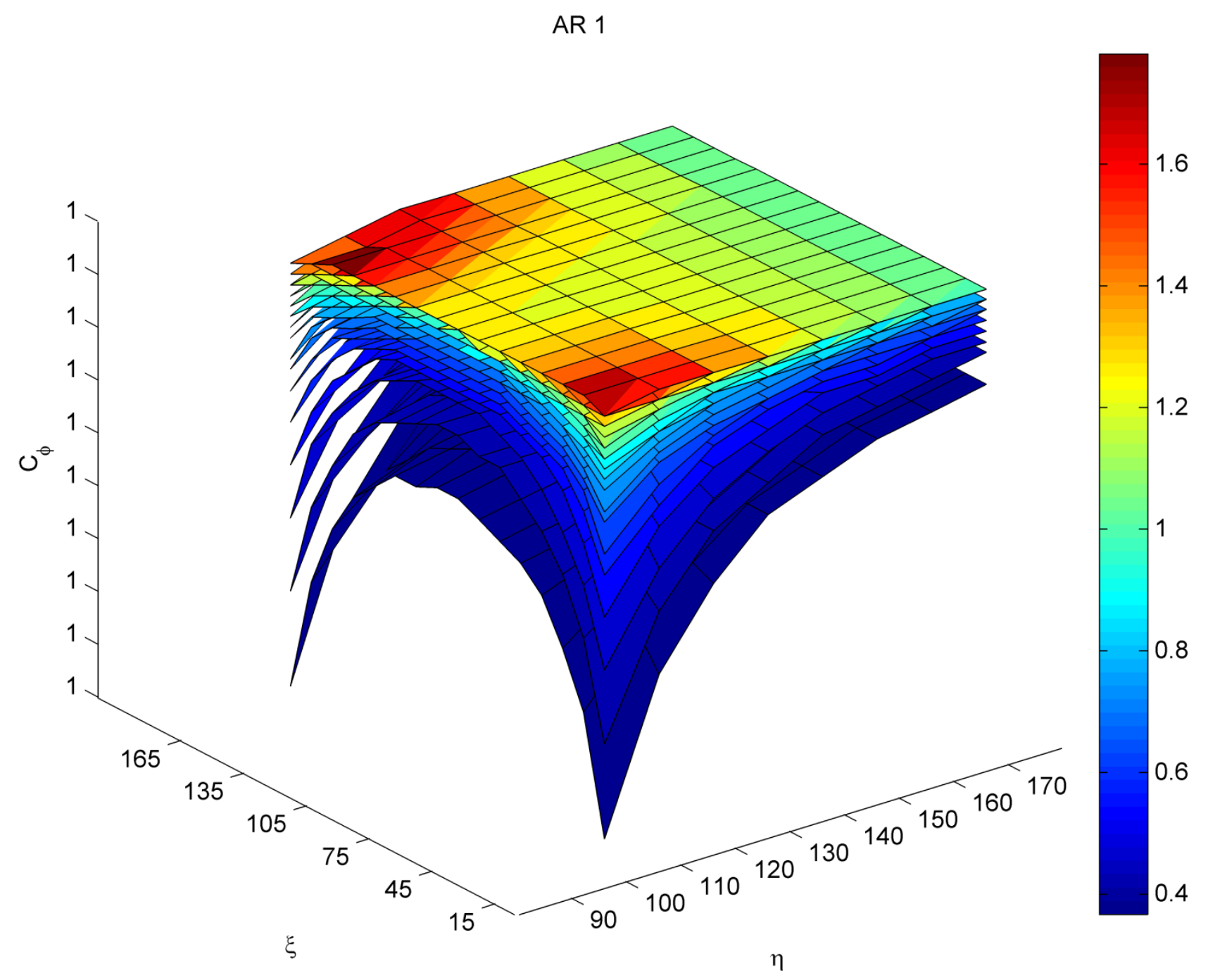

Figure $42 c_{\phi}$ for $A R 1$ 


\section{AR 1}

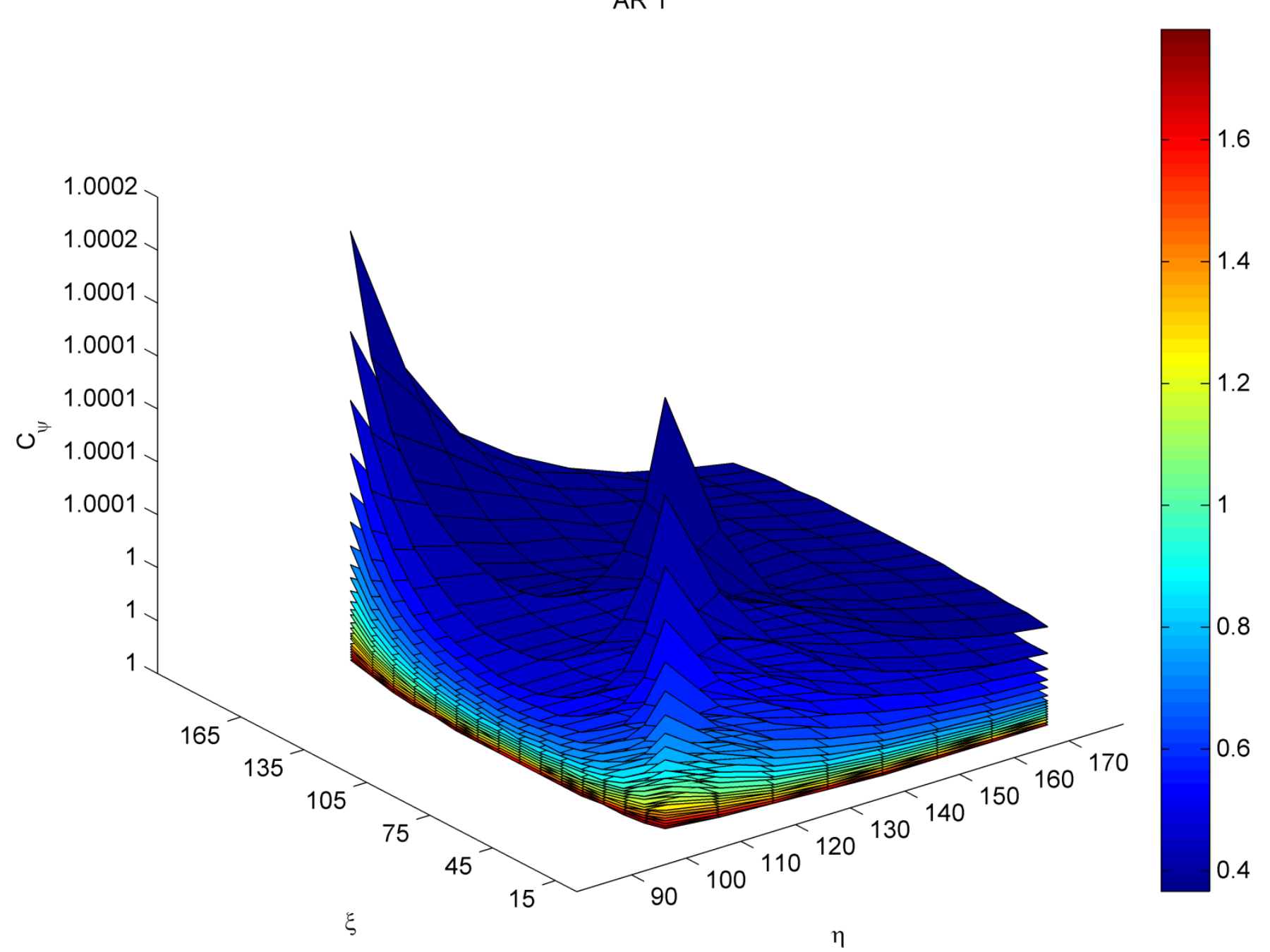

Figure $43 c_{\psi}$ for $A R 1$ 
AR 1

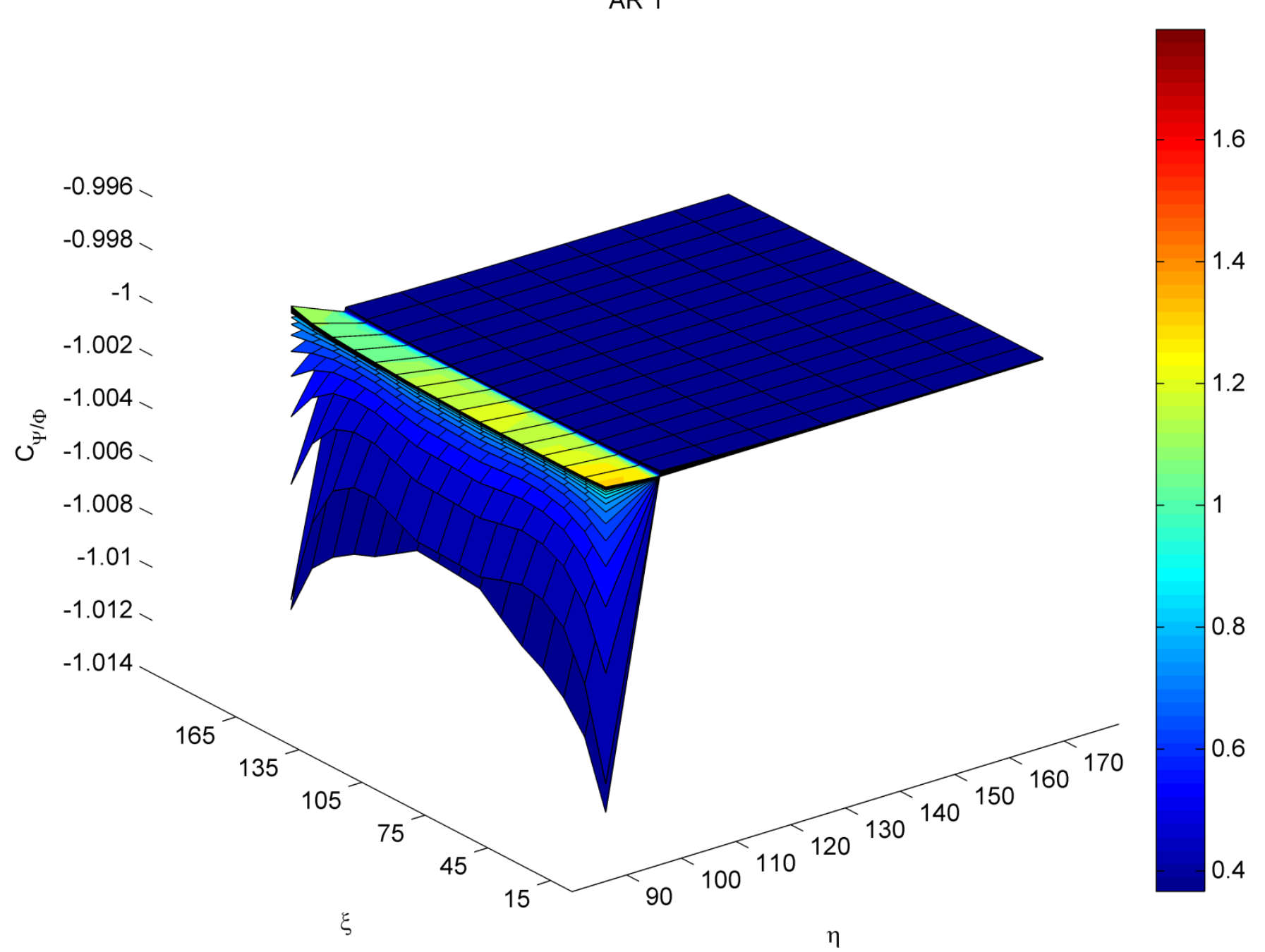

Figure $44 c_{\Psi / \Phi}$ for $A R 1$ 


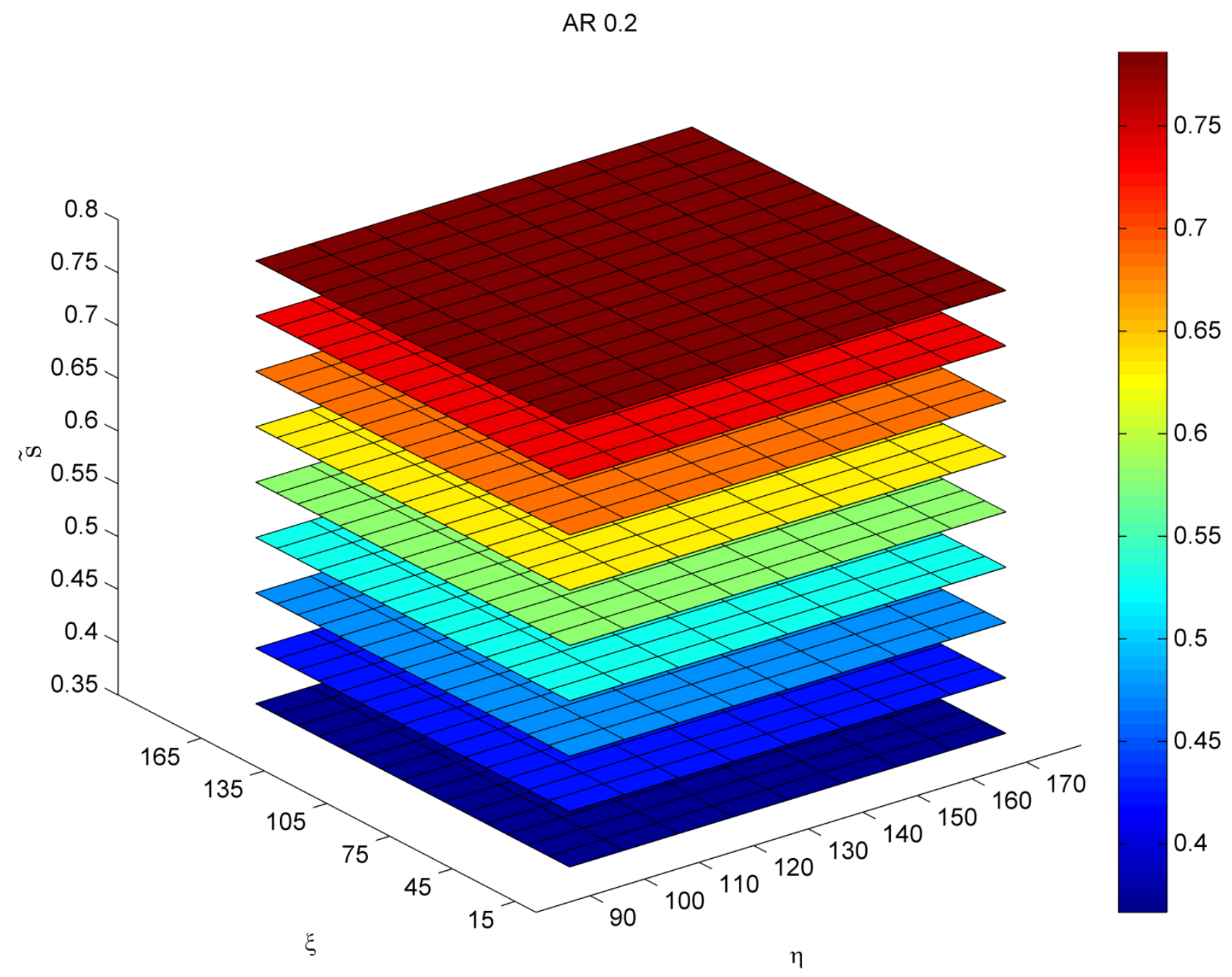

Figure 45 Nondimensional length for $A R 0.2$ 


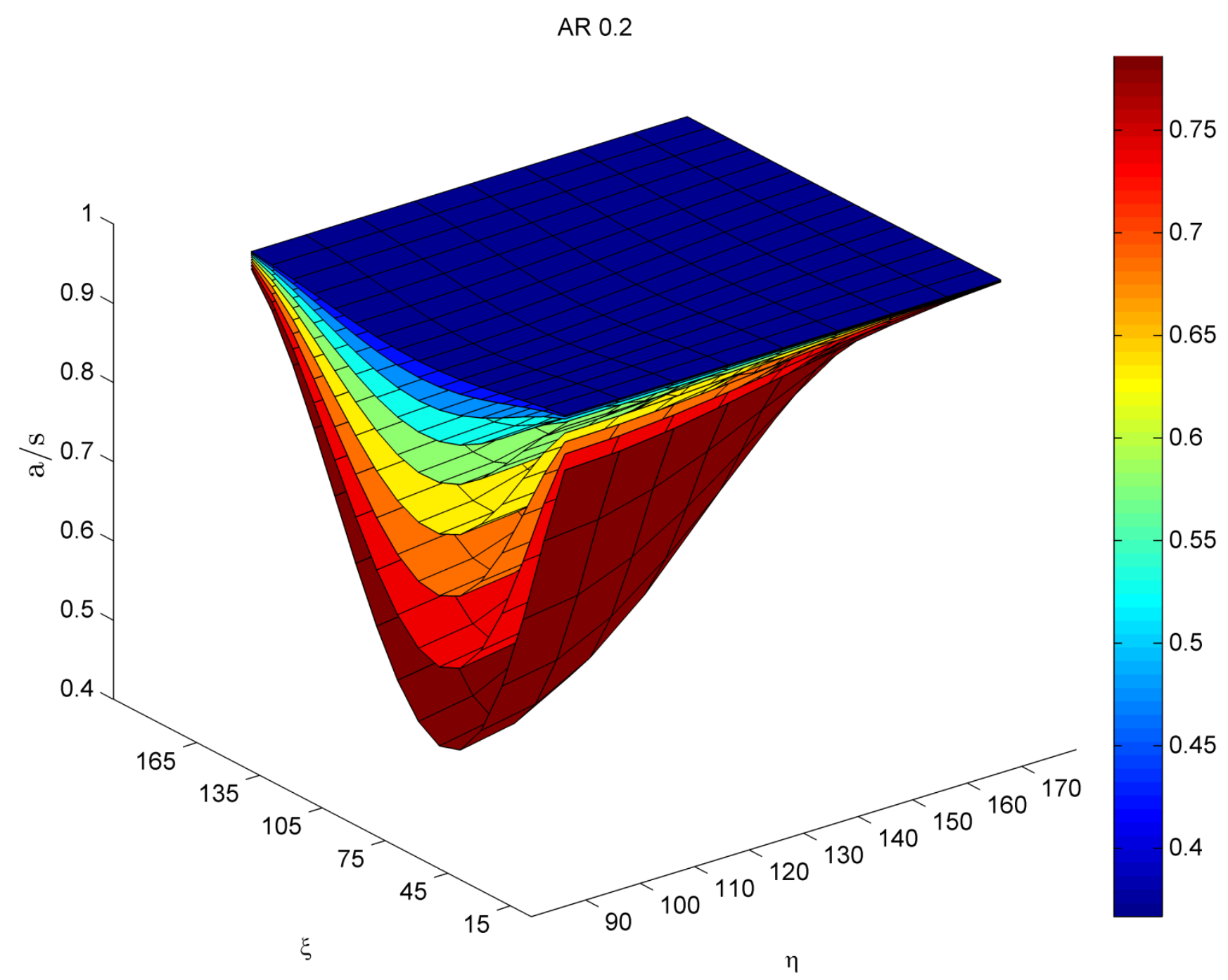

Figure $46 a / s$ for $A R 0.2$ 


\section{AR 0.2}

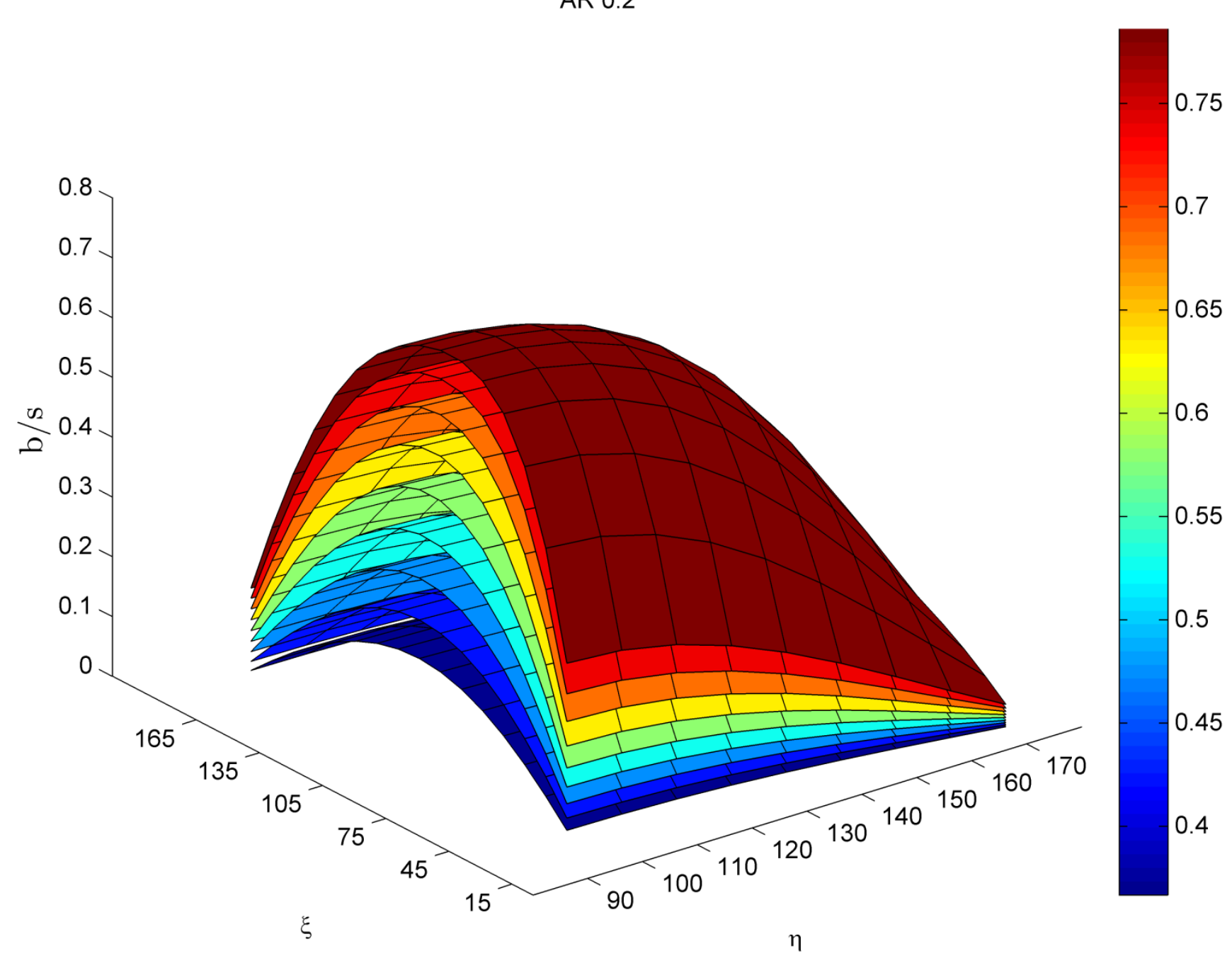

Figure $47 \mathrm{~b} / \mathrm{s}$ for $A R 0.2$ 


\section{AR 0.2}

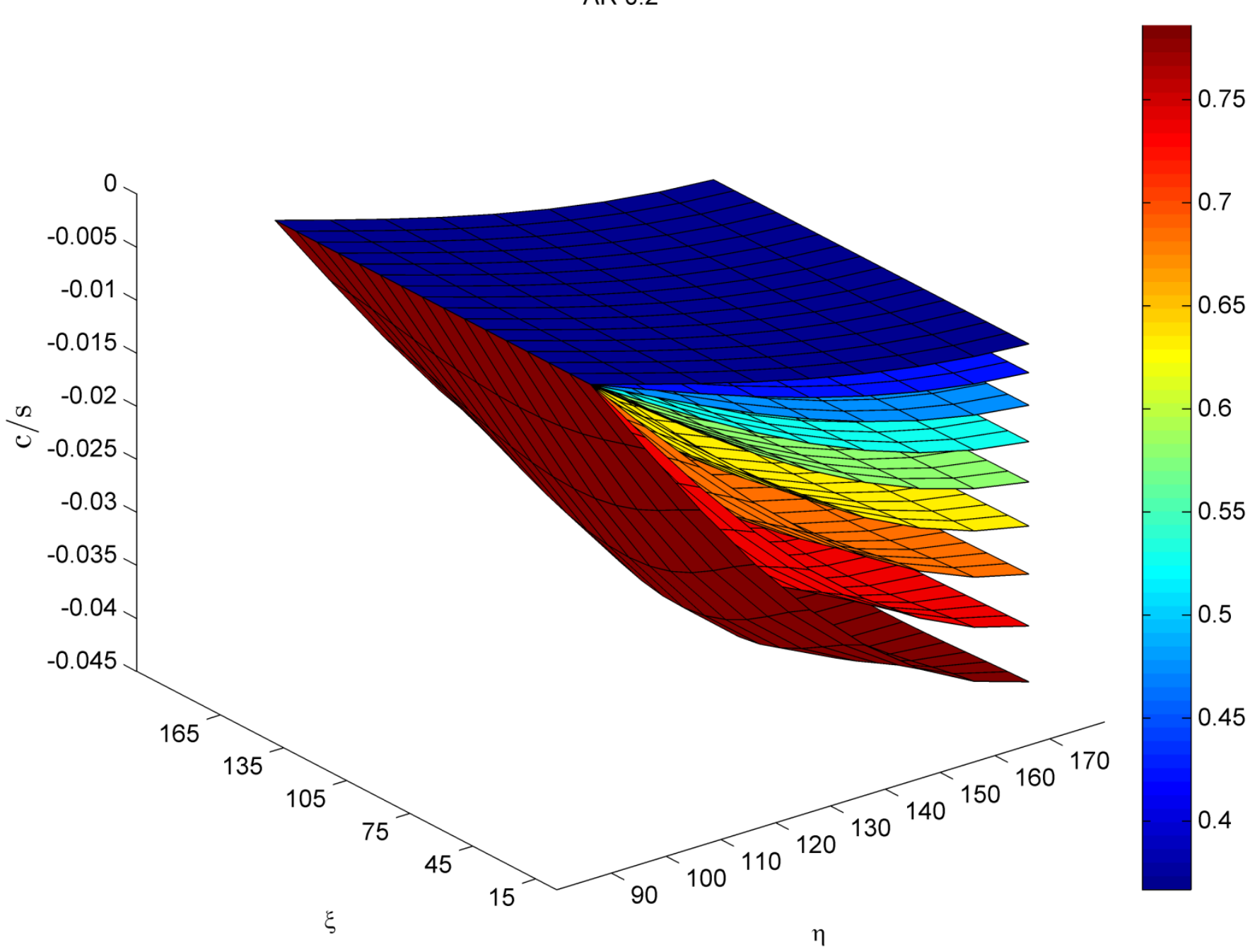

Figure $48 c / s$ for $A R 0.2$ 
AR 0.2

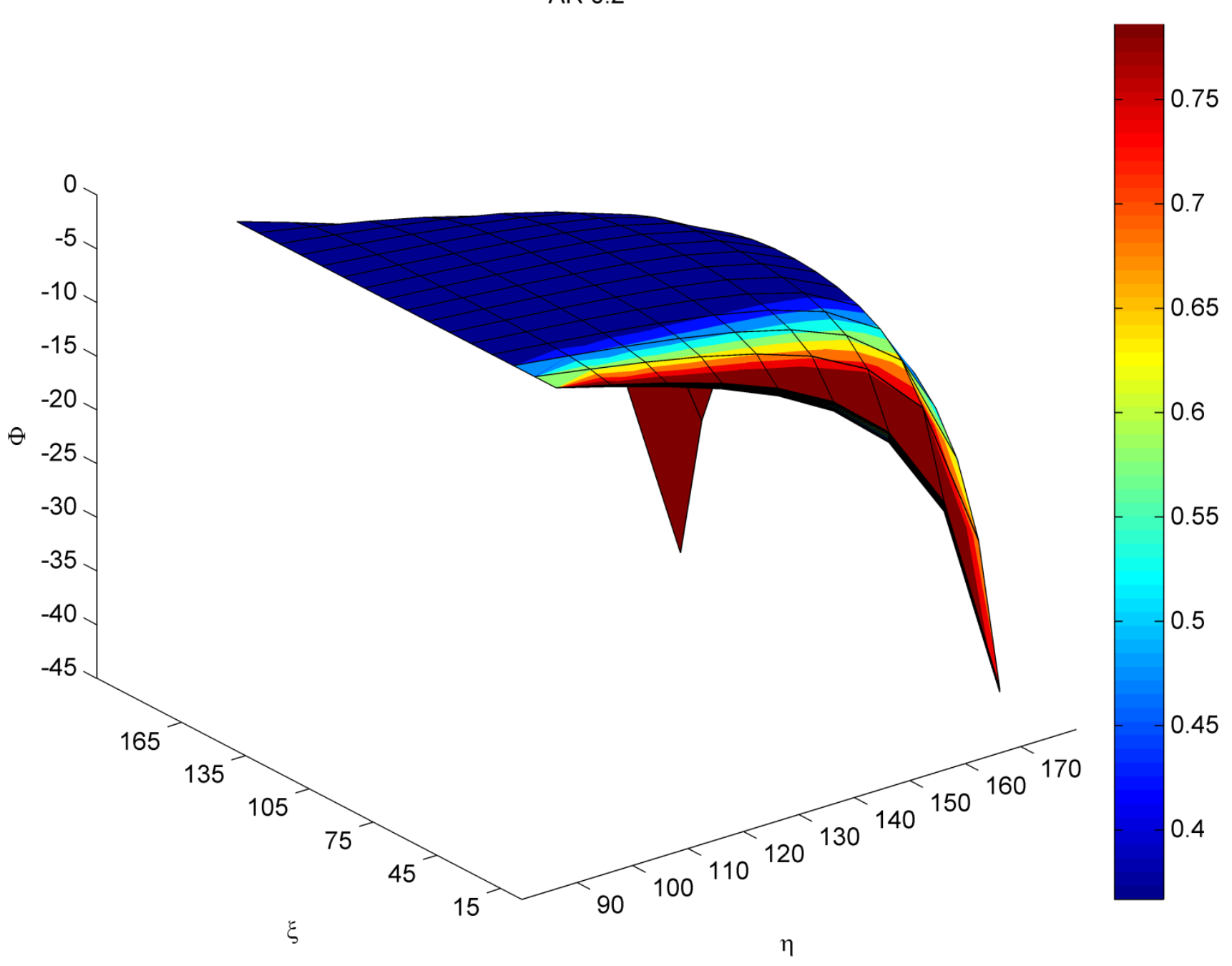

Figure $49 \Phi$ for $A R 0.2$ 


\section{AR 0.2}

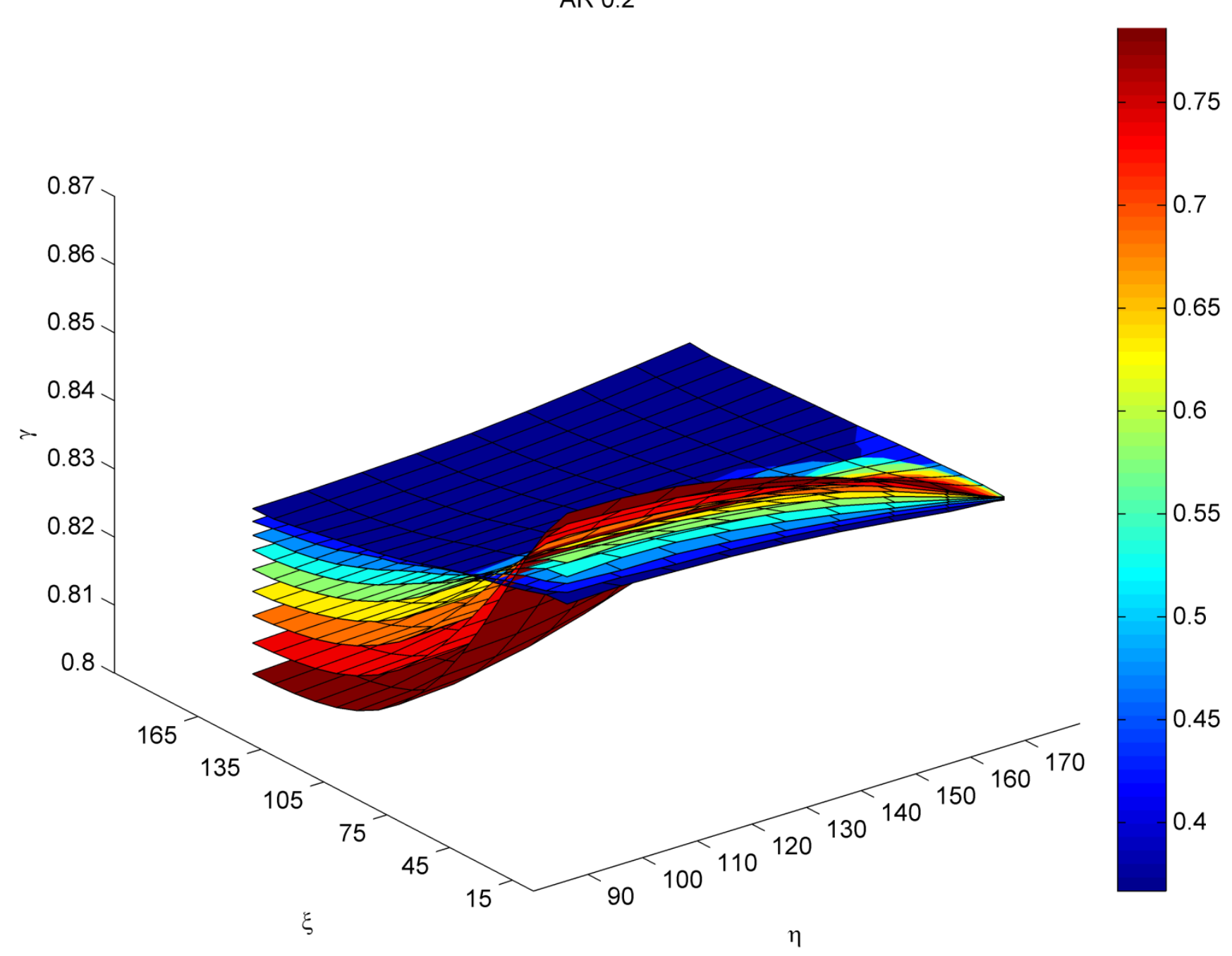

Figure $50 \gamma$ for $A R 0.2$ 
AR 0.2

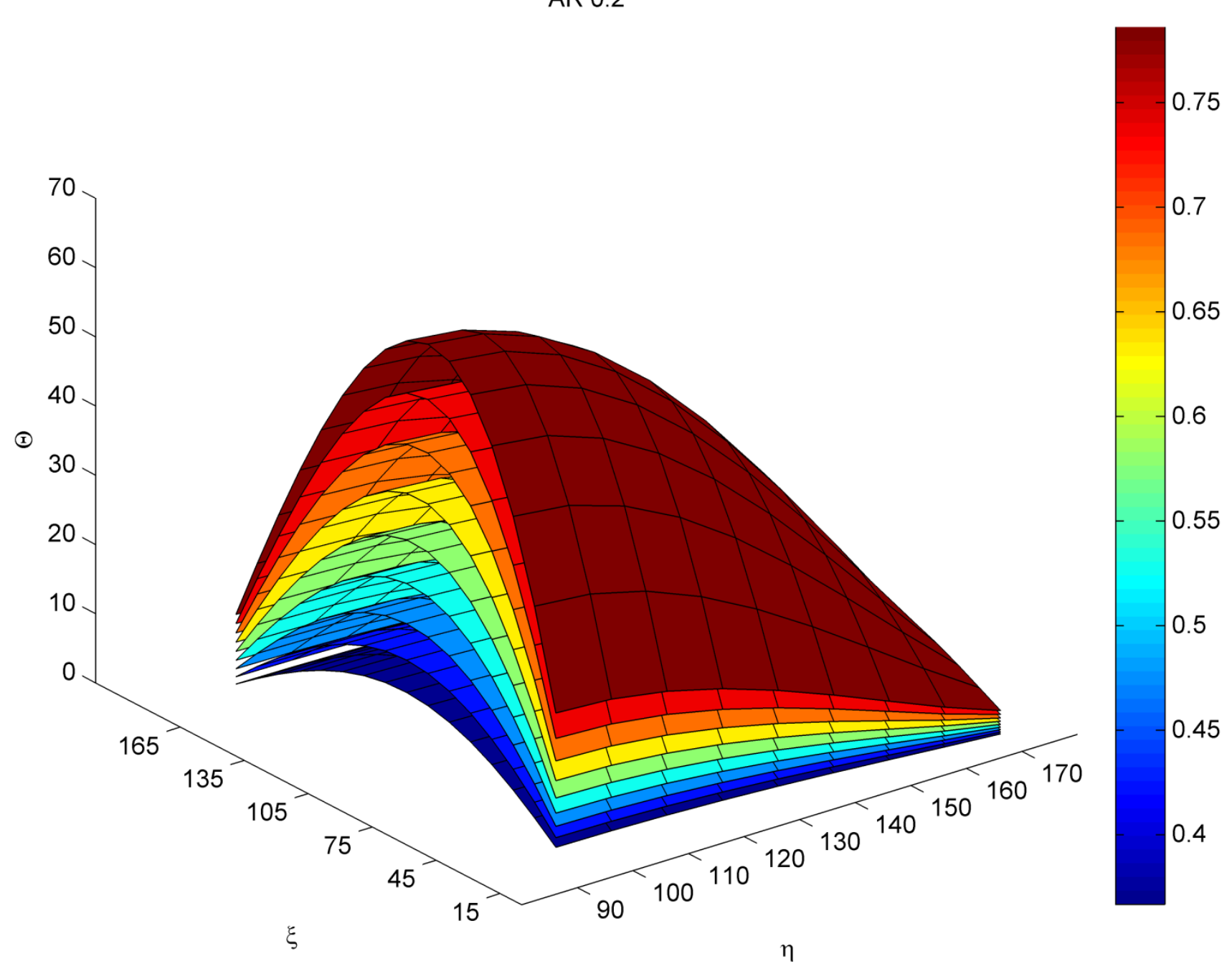

Figure $51 \Theta$ for $A R 0.2$ 
AR 0.2

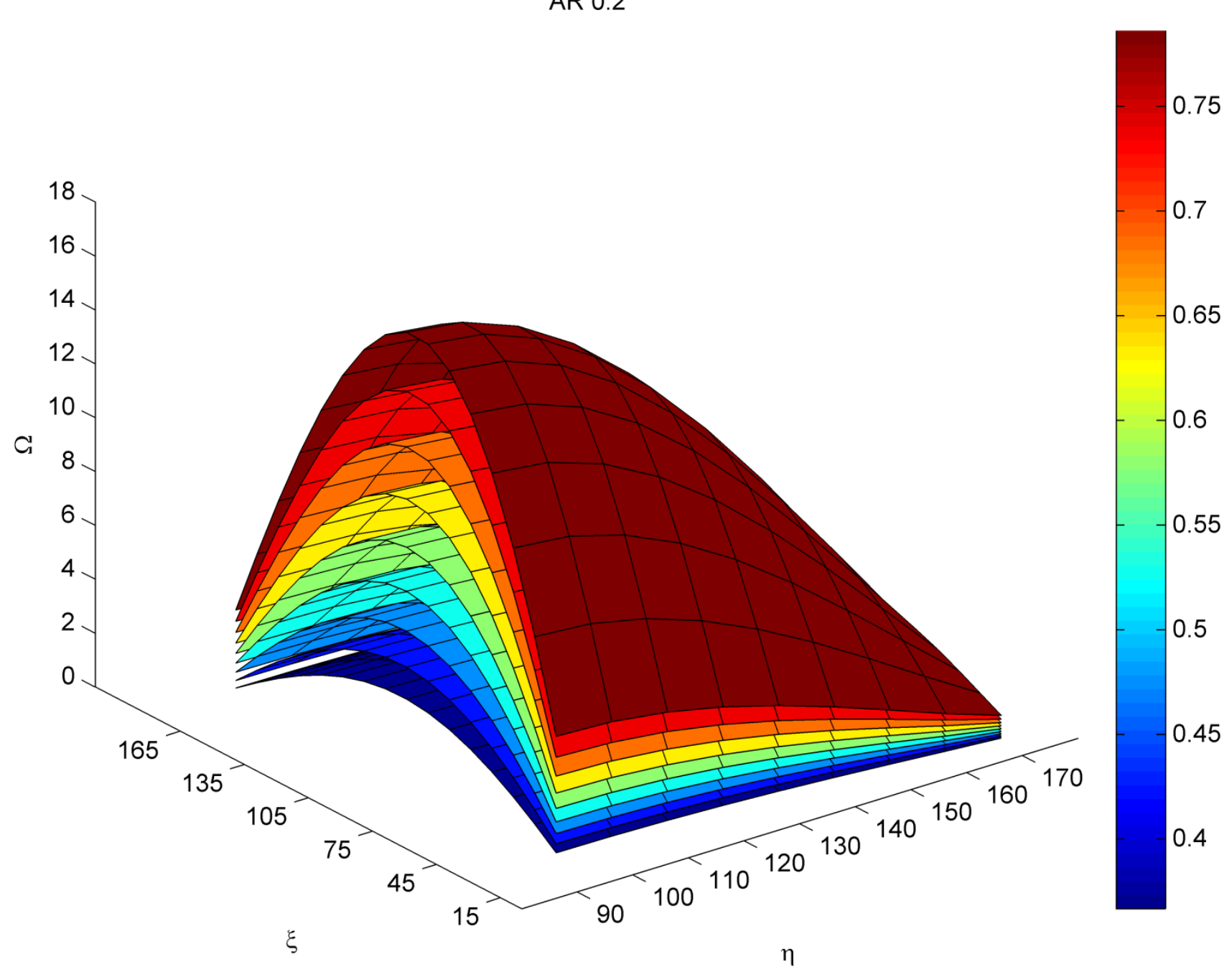

Figure $52 \Omega$ for $A R 0.2$ 


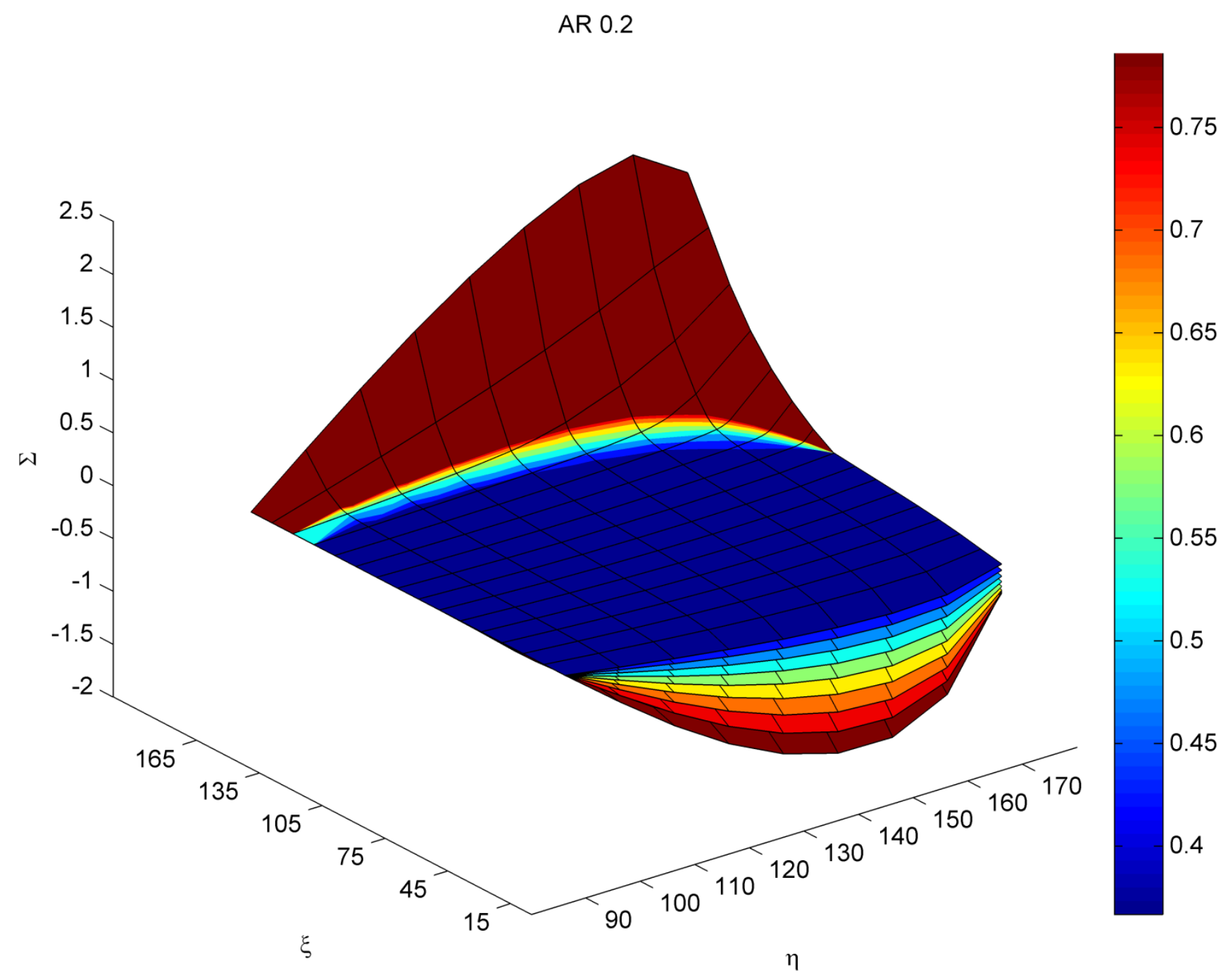

Figure $53 \Sigma$ for $A R 0.2$ 
AR 0.2

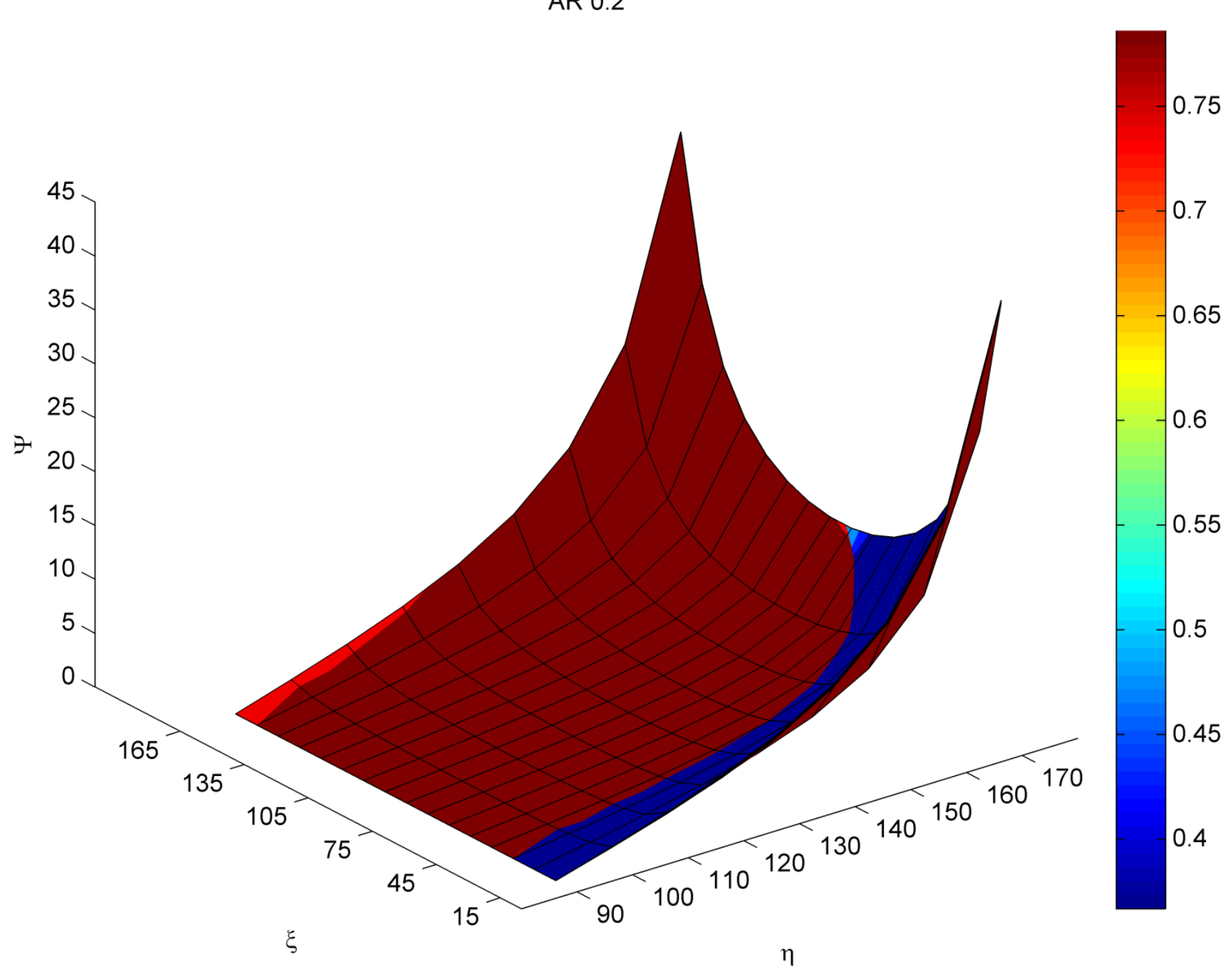

Figure $54 \Psi$ for $A R 0.2$ 


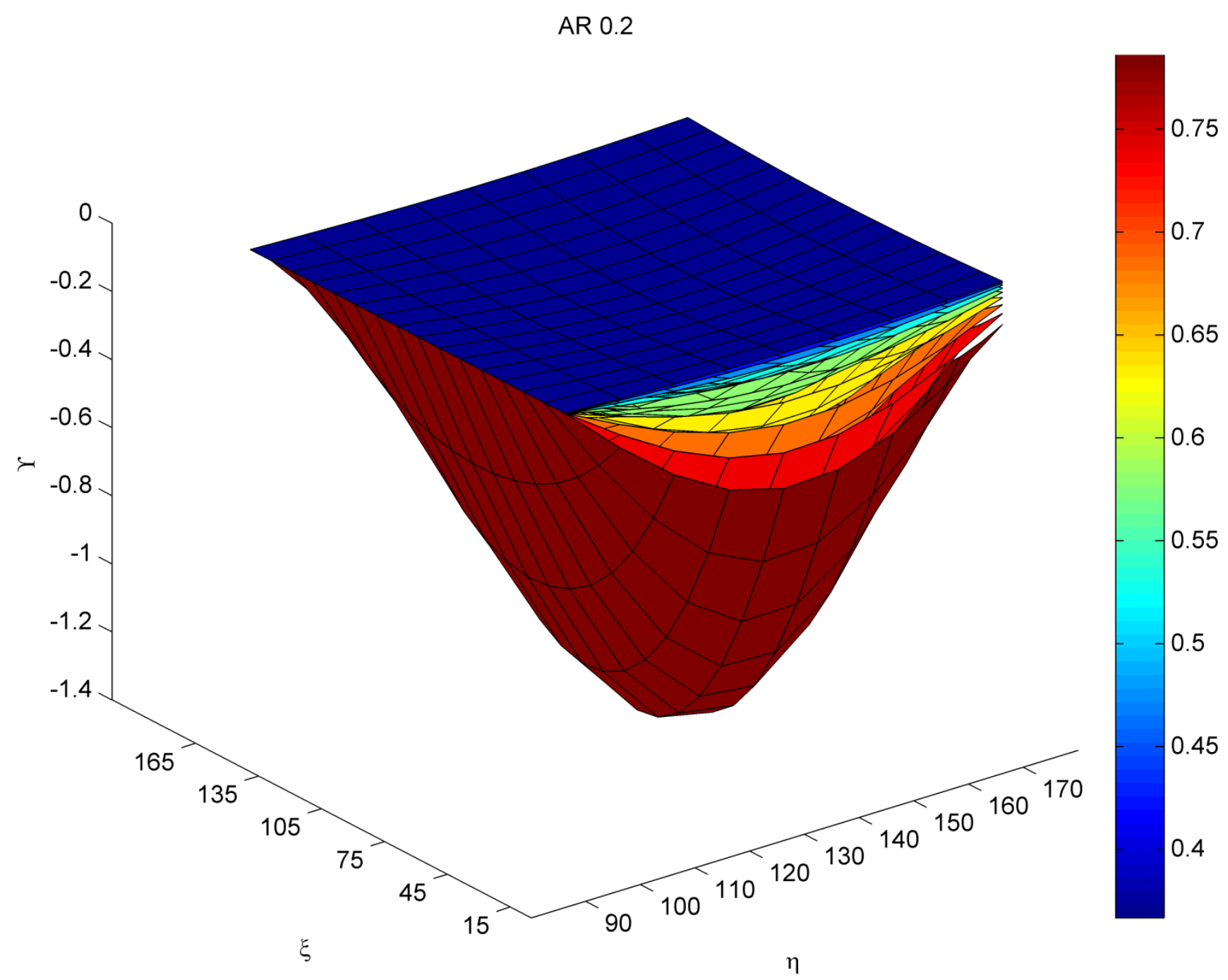

Figure $55 \Upsilon$ for $A R 0.2$ 
AR 0.2

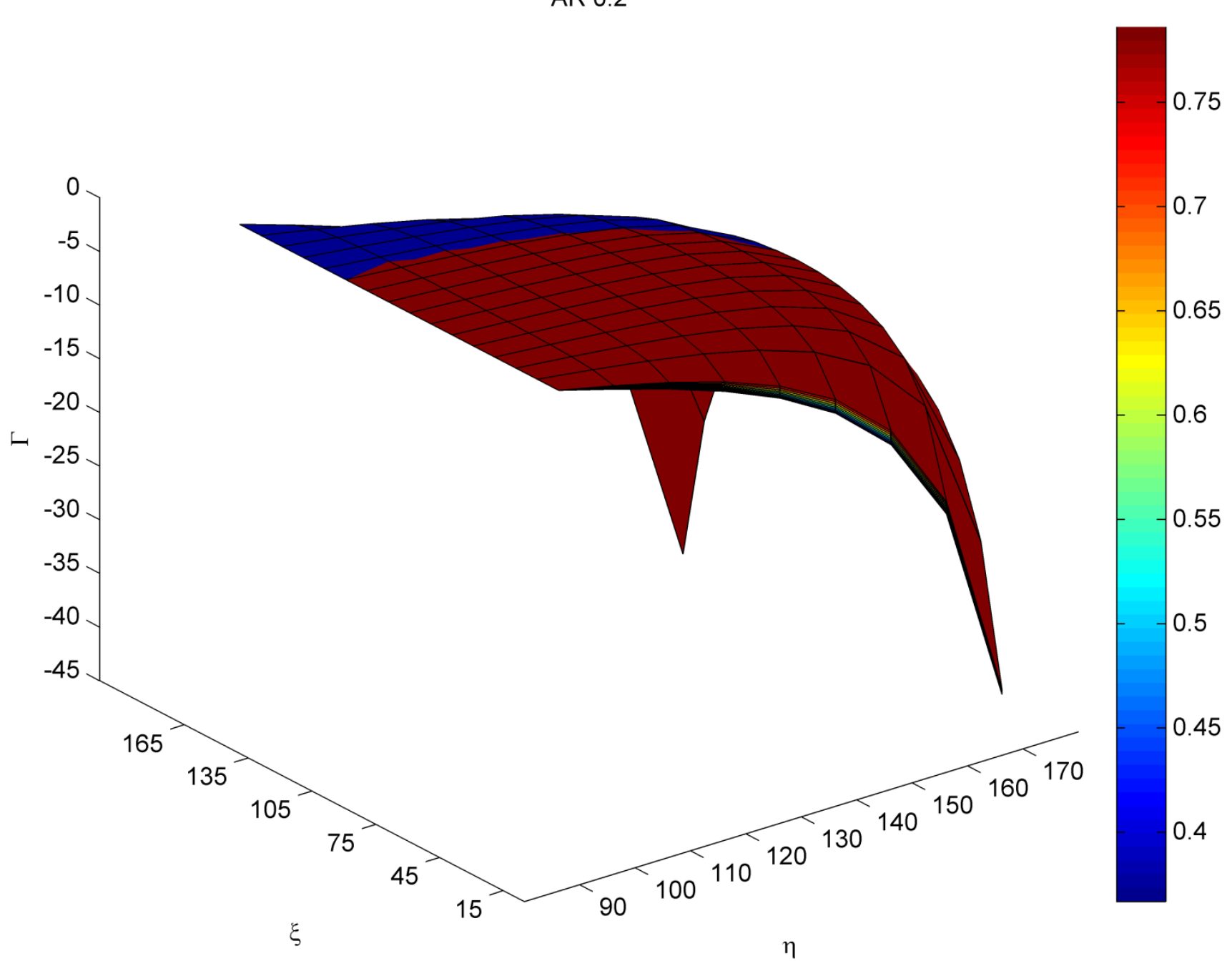

Figure $56 \Gamma$ for $A R 0.2$ 
AR 0.2

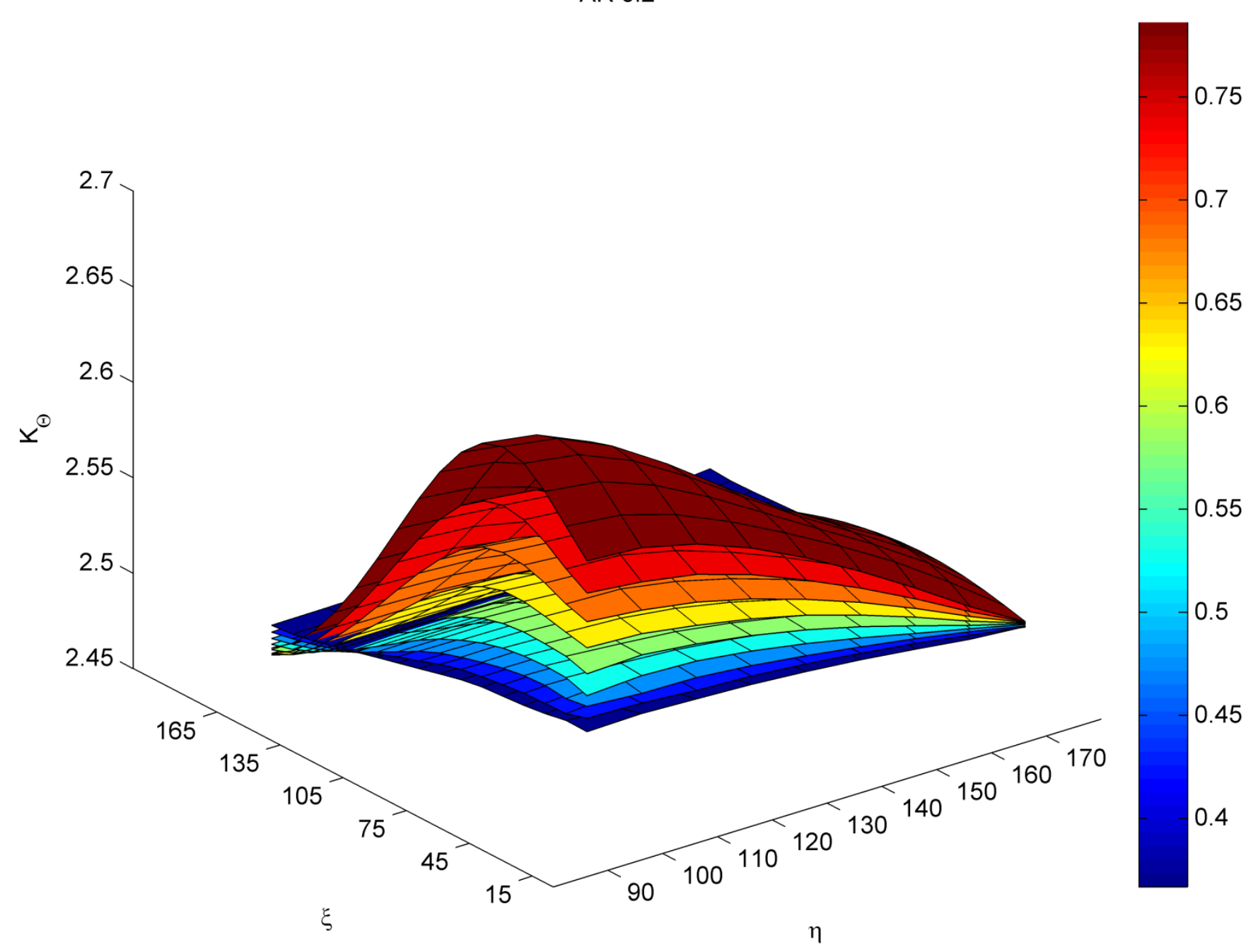

Figure $57 K_{\Theta}$ for $A R 0.2$ 


\section{AR 0.2}

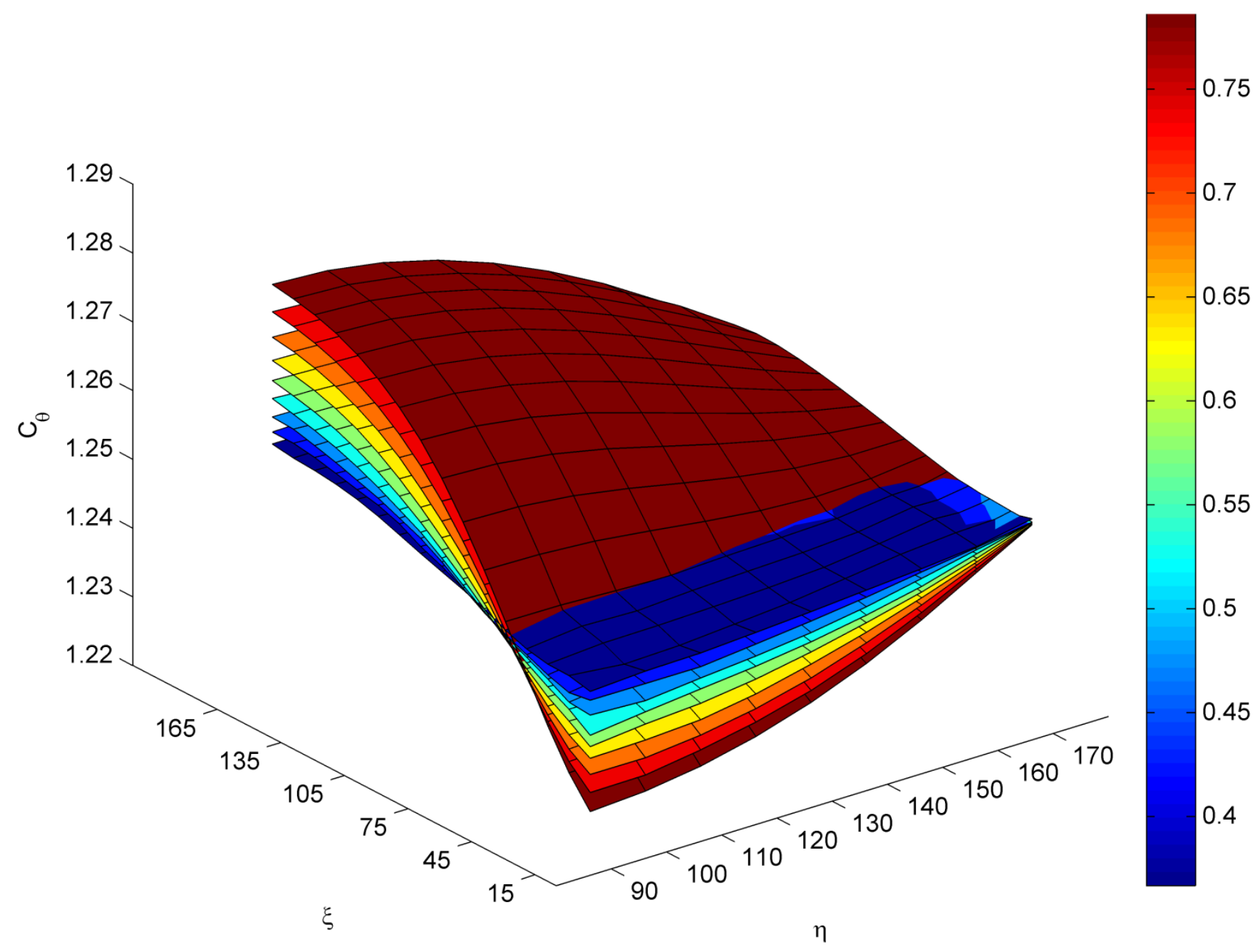

Figure $58 c_{\theta}$ for $A R 0.2$ 
AR 0.2

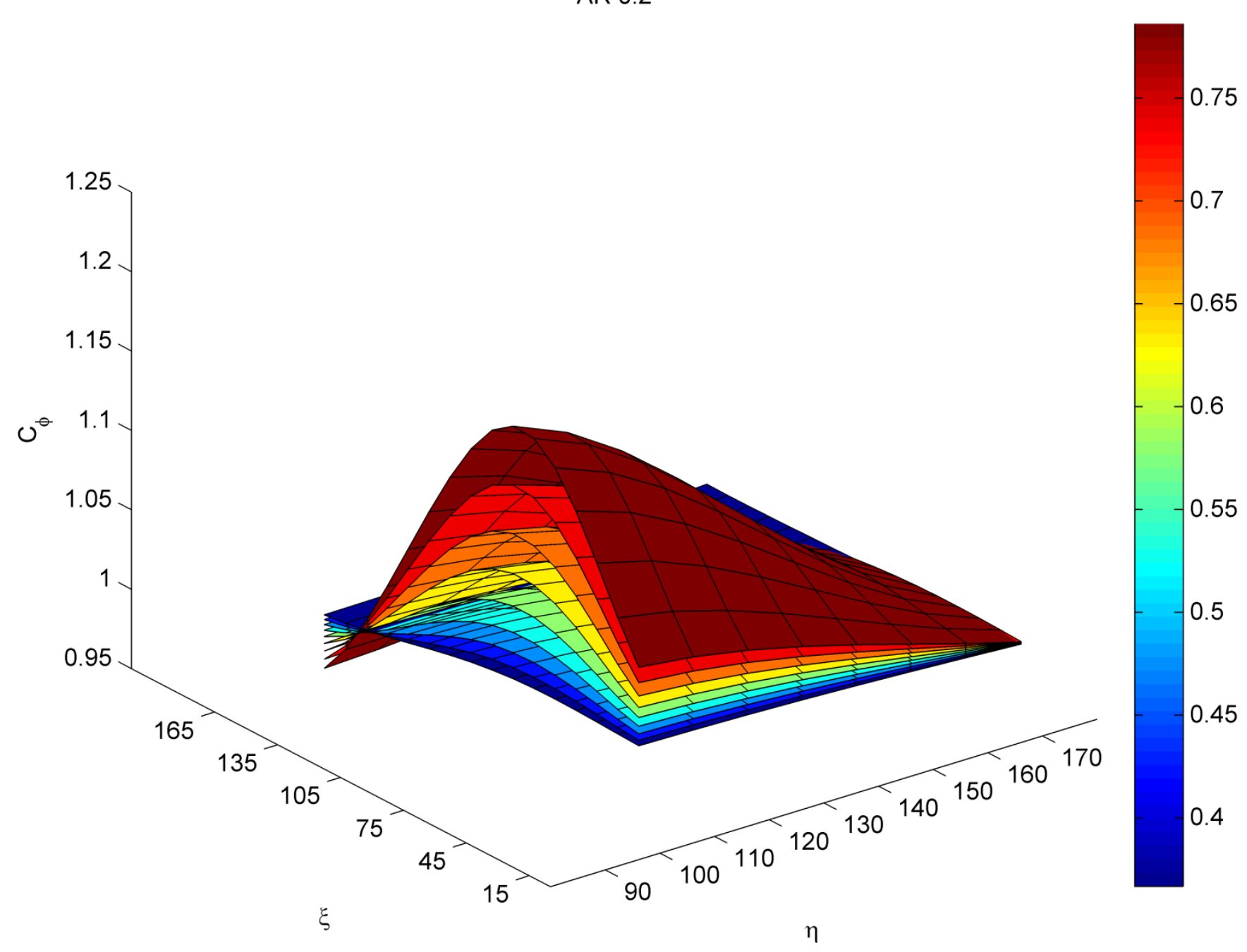

Figure $59 c_{\phi}$ for $A R 0.2$ 
AR 0.2

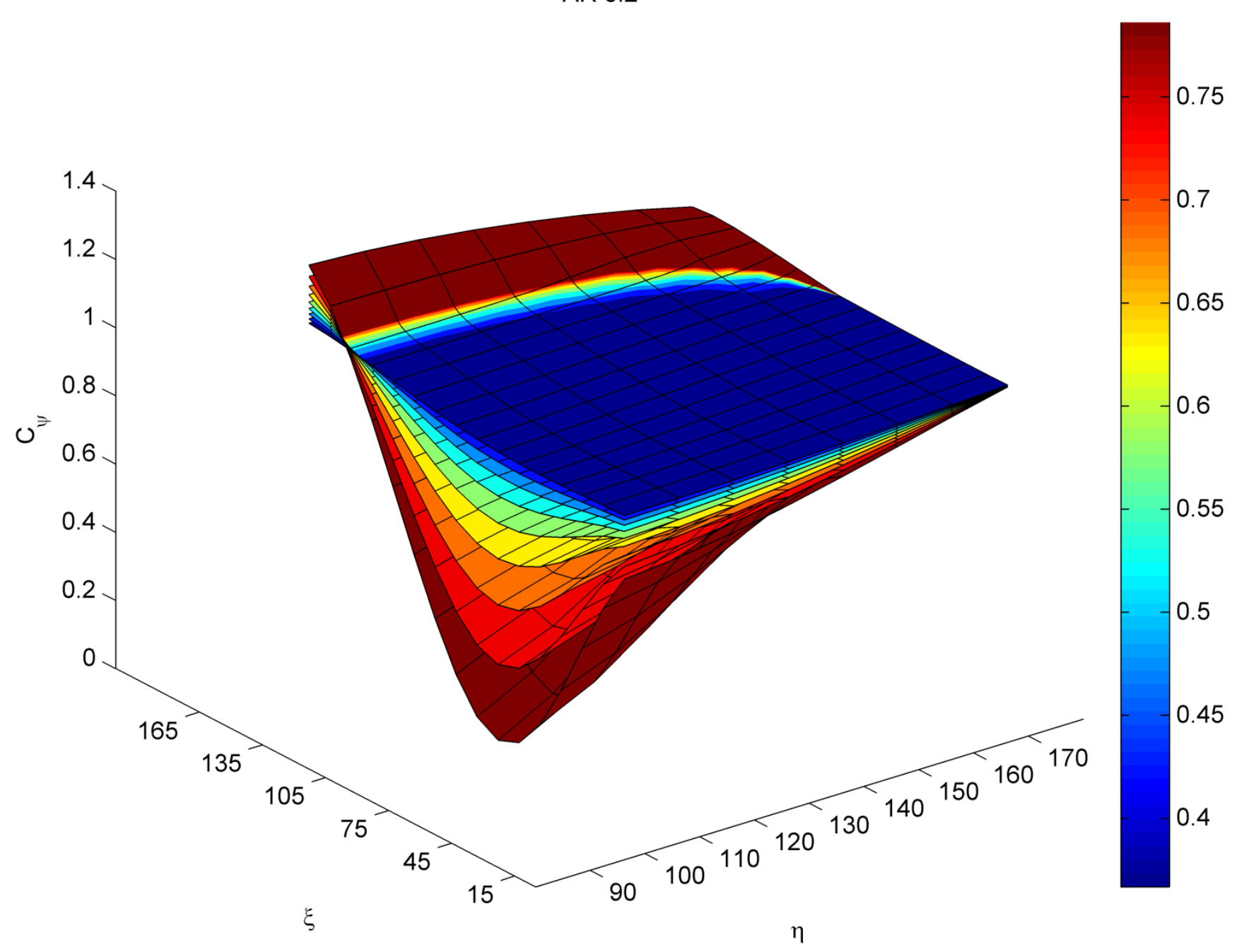

Figure $60 c_{\psi}$ for $A R 0.2$ 
AR 0.2

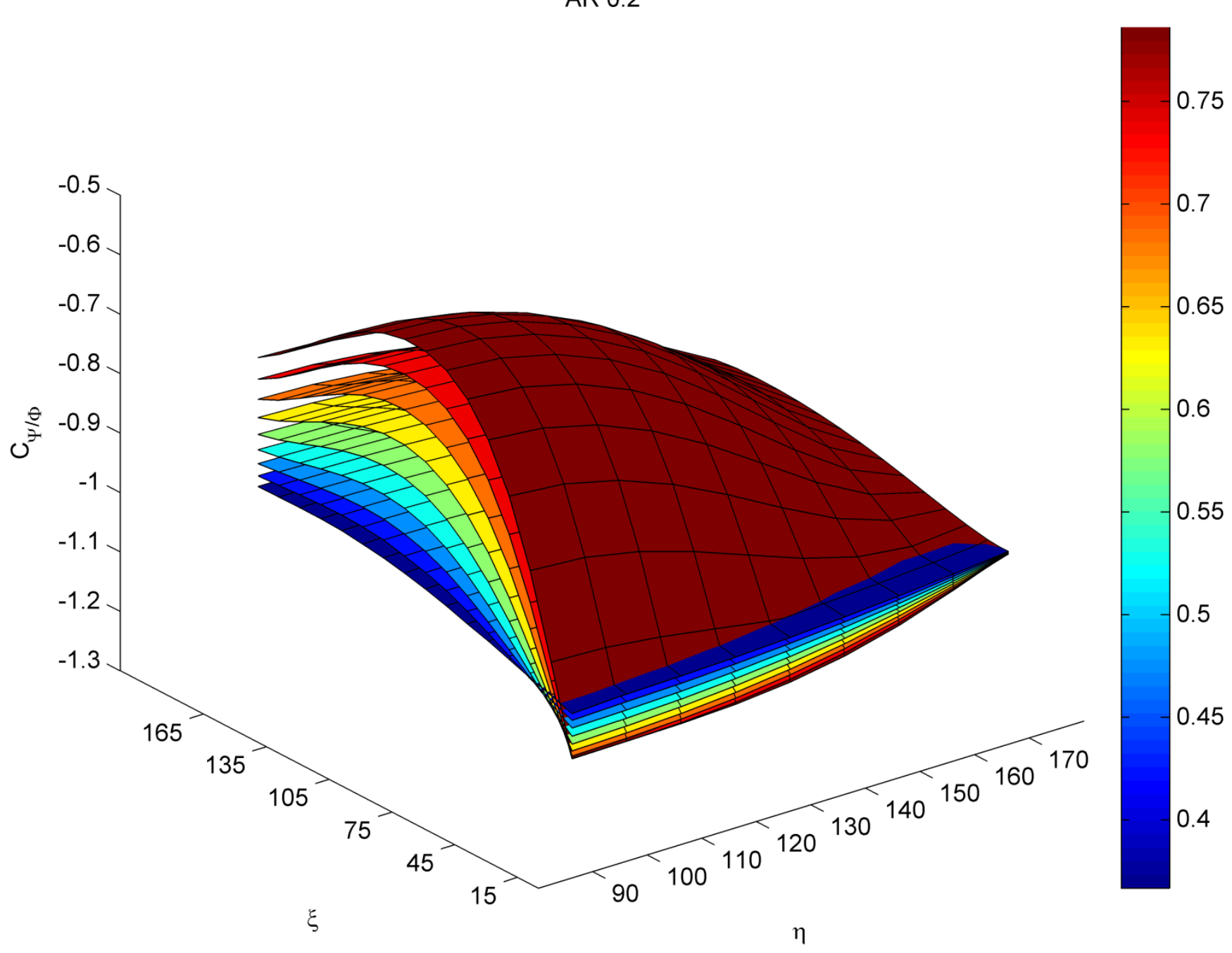

Figure $61 c_{\Psi / \Phi}$ for $A R 0.2$ 


\section{CHAPTER 7: EXAMPLES}

In order to demonstrate the application of the model, a spatial slider-crank mechanism is analyzed. This mechanism is recognized as revolute-spherical-spherical-prismatic (RSSP). The kinematic analysis of the mechanism is based in the rigid RSSP, as presented by Parlaktas [33].

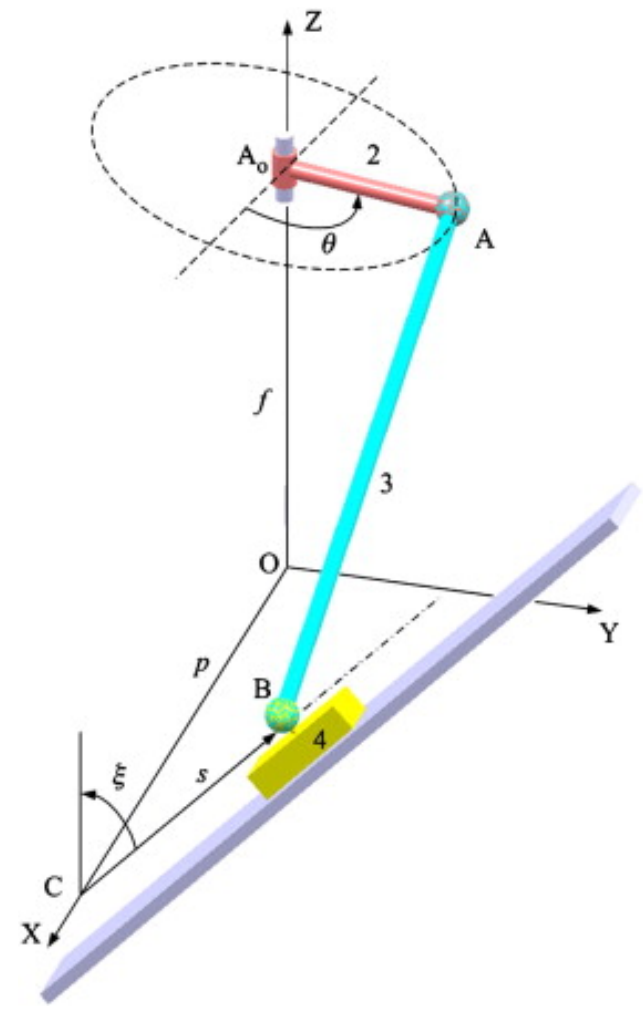

Figure 62 Spatial slider-crank mechanism.

Figure 62 shows the angle and length parameters used to construct the mechanism, and they are defined in Table 4. The position vector loop is presented in equation (121), and provides three equations, three unknowns $(\Phi, \Theta, s)$ system: 
Table 4 RSSP parameters

\begin{tabular}{|l|l|l|}
\hline Parameter Name & Description & Value \\
\hline $\mathrm{l} 2$ & Crank length & $25 \mathrm{~mm}$ \\
\hline $\mathrm{l} 3$ & Coupler length & $150 \mathrm{~mm}$ \\
\hline $\mathrm{p}$ & Distance of the common perpendicular $|\mathrm{OC}|$ & $20 \mathrm{~mm}$ \\
\hline $\mathrm{f}$ & Distance of crank elevation $|\mathrm{OAo}|$ & $129 \mathrm{~mm}$ \\
\hline$\theta$ & Crank angle & $0^{\circ}-360^{\circ}$ \\
\hline$\xi$ & Angular offset & $80^{\circ}$ \\
\hline $\mathrm{s}$ & Slider position & variable \\
\hline
\end{tabular}

$$
f^{z}[I]+L_{2}{ }^{y}\left[R_{x}(\theta) I\right]=p^{x}[I]+s^{z}\left[R_{x}(-\xi) I\right]+L_{3}{ }^{y}\left[R_{x}(-\xi) R_{y}(\Phi) R_{x}(\Theta) I\right]
$$

where, $I$ is the identity matrix, $\Phi$ and $\Theta$ are pseudo rigid body angles. This equation expands in the following three components:

$$
\begin{gathered}
L_{3} \sin (\Phi) \sin (\xi)-L_{2} \sin (\theta)-p=0 \\
L_{2} \cos (\theta)-L_{3}[\cos (\Theta) \cos (\xi)+\cos (\Phi) \sin (\Theta) \sin (\xi)]-s * \sin (\xi)=0 \\
f-L_{3}[\sin (\Theta) \cos (\xi)-\cos (\Phi) \cos (\Theta) \sin (\xi)]-s * \sin (\xi)
\end{gathered}
$$

Using equation (58), and combining equations (74) and (75) the following system solves for PRMB angles $\Gamma$ and $\Upsilon$.

$$
\begin{gathered}
\Phi=\Upsilon+\Gamma \\
\frac{{ }^{\{C\}} I_{x x} G}{(1-\gamma) L} \Upsilon=\gamma K_{\Theta} \frac{E}{L} I_{R} \Theta^{2} \sin (2 \Gamma)
\end{gathered}
$$

Partial differentiation of equations (122)-(124) with respect to $\theta$ yields another 3x3 system of equations solving for $\left(\frac{\partial \Phi}{\partial \theta}, \frac{\partial \Theta}{\partial \theta}, \frac{\partial \mathrm{s}}{\partial \theta}\right)$ 


$$
\begin{gathered}
L_{3} \cos (\Phi) \sin (\xi) \frac{\partial \Phi}{\partial \theta}-L_{2} \cos (\theta)=0 \\
L_{3}[\sin (\Theta) \cos (\xi)+\cos (\Phi) \cos (\Theta) \sin (\xi)] \frac{\partial \Theta}{\partial \theta}-L_{2} \sin (\theta)-\sin (\xi) \frac{\partial \mathrm{s}}{\partial \theta} \\
+L_{3} \sin (\Phi) \sin (\Theta) \sin (\xi) \frac{\partial \Phi}{\partial \theta}=0 \\
L_{3}[\cos (\Theta) \cos (\xi)+\cos (\Phi) \sin (\Theta) \sin (\xi)] \frac{\partial \Theta}{\partial \theta}+\cos (\xi) \frac{\partial \mathrm{s}}{\partial \theta} \\
+L_{3} \sin (\Phi) \cos (\Theta) \sin (\xi) \frac{\partial \Phi}{\partial \theta}=0
\end{gathered}
$$

Differentiation of equations (58) and (125) with respect of $\theta$ yields a $2 \times 2$ system to find $\left(\frac{\partial \Upsilon}{\partial \theta}, \frac{\partial \Gamma}{\partial \theta}\right)$

$$
\begin{gathered}
\frac{\partial \Phi}{\partial \theta}=\frac{\partial \Upsilon}{\partial \theta}+\frac{\partial \Gamma}{\partial \theta} \\
\frac{\left\{C I_{x x} G\right.}{(1-\gamma) L} \frac{\partial \Upsilon}{\partial \theta}=\gamma K_{\Theta} \frac{E}{L} I_{R}\left[2 \Theta \sin (2 \Gamma) \frac{\partial \Theta}{\partial \theta}+2 \Theta^{2} \cos (2 \Gamma) \frac{\partial \Gamma}{\partial \theta}\right]
\end{gathered}
$$

Using the principle of virtual work for the RSSR mechanisms, an expression of the torque related to the crack angle was obtained:

$$
\begin{gathered}
\frac{\partial V_{T}}{\partial \theta}=\frac{\{C\} I_{x x} G}{(1-\gamma) L} \Upsilon \frac{\partial \Upsilon}{\partial \theta} \\
\frac{\partial V_{B}}{\partial \theta}=\gamma K_{\Theta} \frac{E}{L}\left[I_{R} \Theta^{2} \sin (2 \Gamma) \frac{\partial \Gamma}{\partial \theta}+\left(\bar{I}-I_{R} \cos (2 \Gamma)\right) \Theta \frac{\partial \Theta}{\partial \theta}\right] \\
T_{2} \delta \theta-\frac{\partial V_{T}}{\partial \theta} \delta \theta-\frac{\partial V_{B}}{\partial \theta} \delta \theta=0 \\
T_{2}=\frac{{ }^{\{C\}} I_{x x} G}{(1-\gamma) L} \gamma \frac{\partial \Upsilon}{\partial \theta}+\gamma K_{\Theta} \frac{E}{L}\left[I_{R} \Theta^{2} \sin (2 \Gamma) \frac{\partial \Gamma}{\partial \theta}+\left(\bar{I}-I_{R} \cos (2 \Gamma)\right) \Theta \frac{\partial \Theta}{\partial \theta}\right]
\end{gathered}
$$


The following figure shows the relationship between the input torque and the crank angle for the mechanism parameters specified in Table 4.

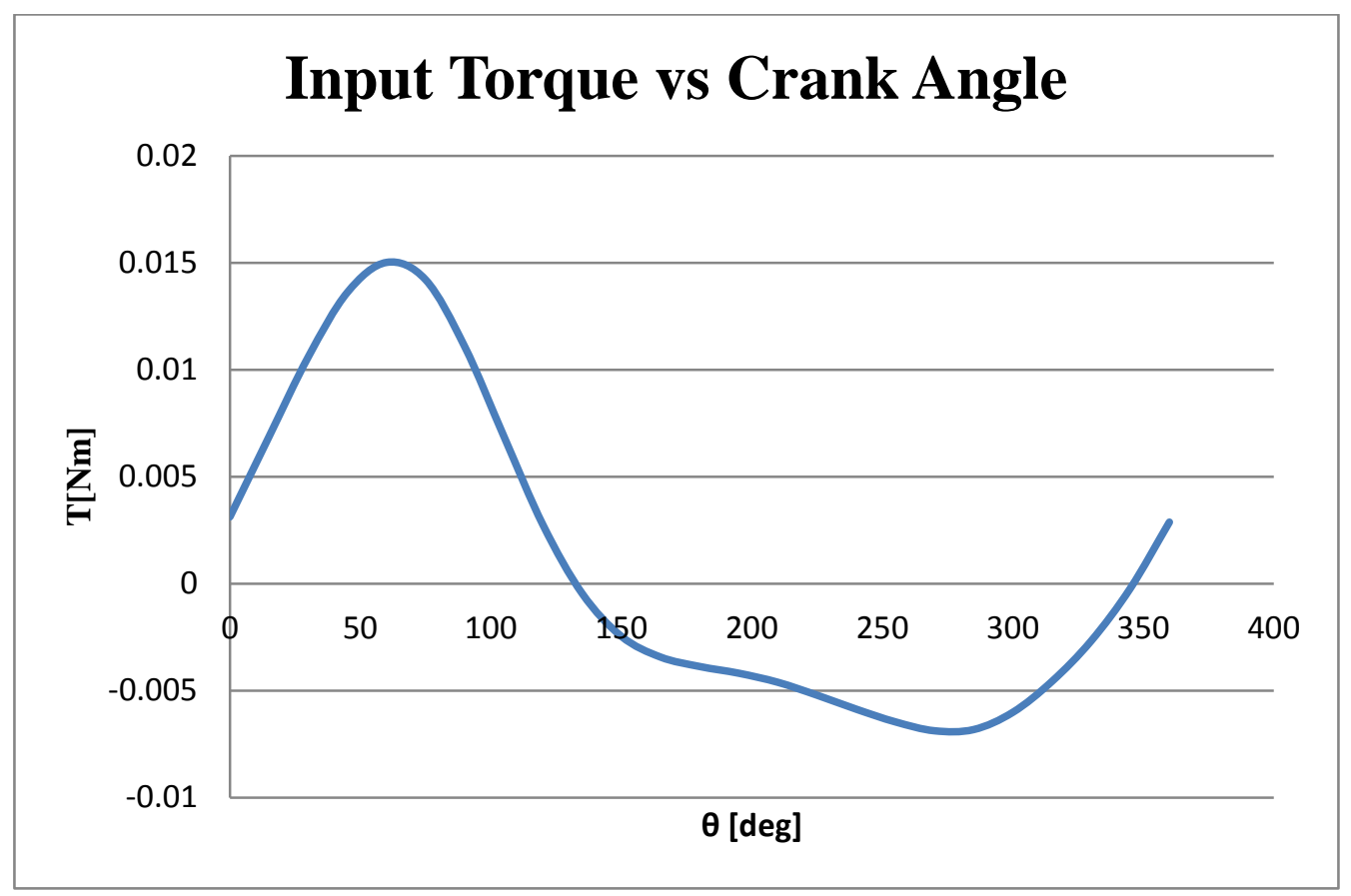

Figure 63 Input torque vs crank angle in RSSP

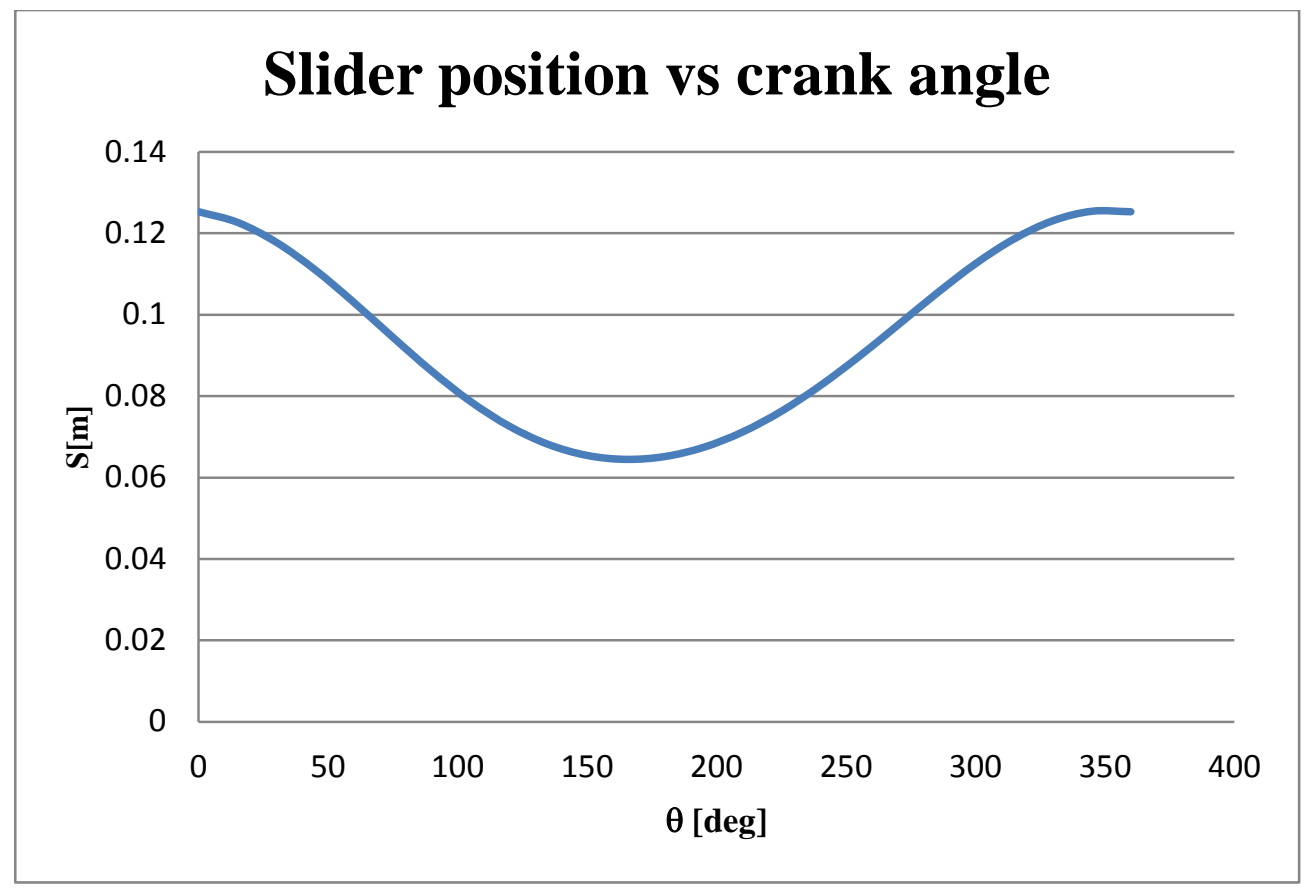

Figure 64 Slider position vs crank angle in RSSP 


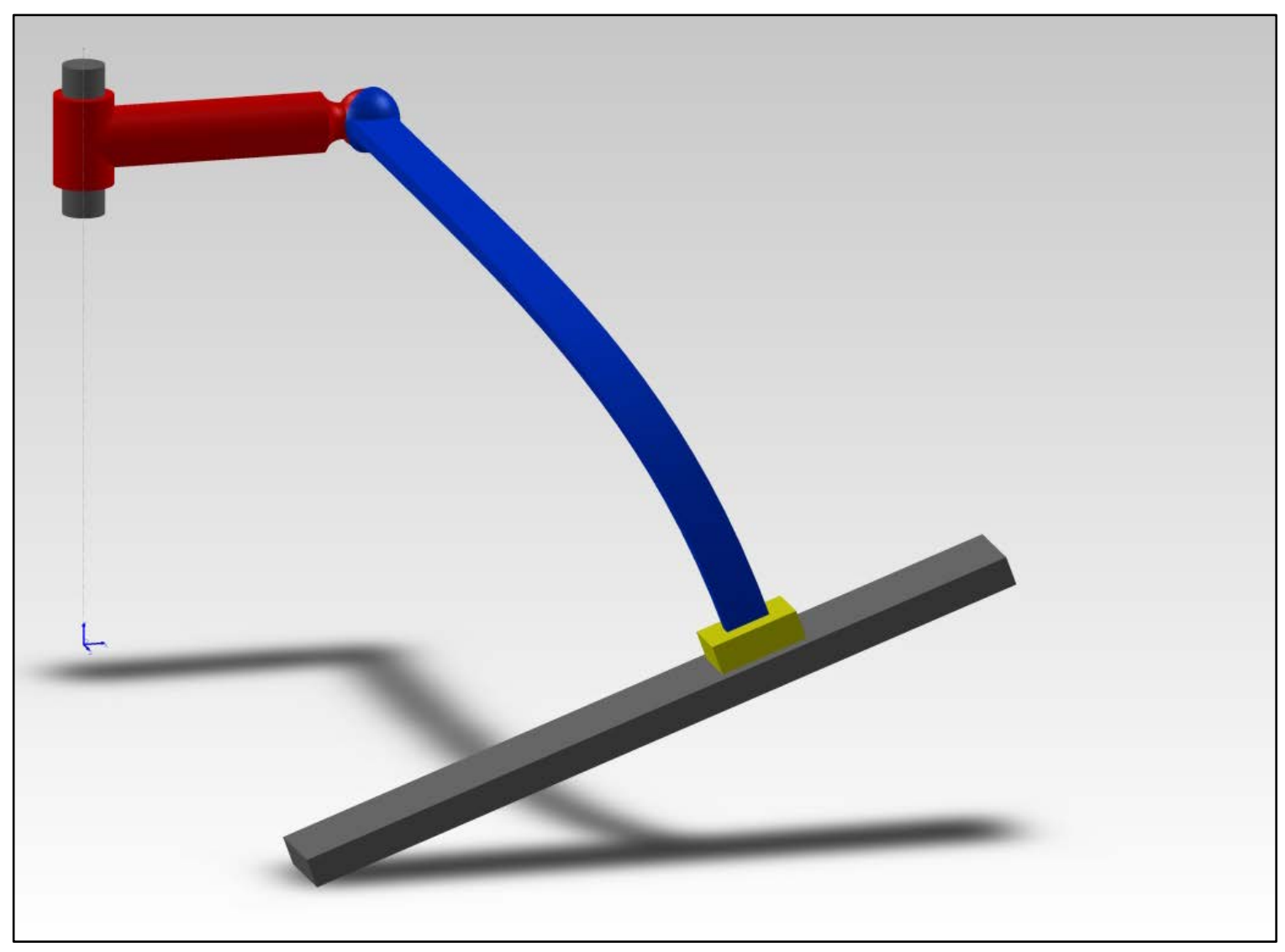

Figure 65 Compliant RSSP

Another example, where the model can be easily applied is in a compliant double slider spatial mechanism where the relative positions are known and the applied force is unknown.

The main difference between a planar and a spatial double slider is that the planes of displacement of both sliders are in different planes. In Figure 66, it can be observed a planar compliant double slider in shaded blue whereas the spatial compliant double slider is solid.

Both sliders are free to move only along the linear slots which orientations are depicted as dotted lines. The compliant member is fixed to the slider in the base along the positive x-axis and is attached to the second slider through a spherical joint. In spherical joints, only forces can be transmitted between connecting members. In the following example, the angle of rotation between the inclined slot and the yaxis is $30^{\circ}$. For this particular application we have the following parameters. 
Table 5 PSSP parameters

\begin{tabular}{|l|l|l|}
\hline Parameter & Value & Description \\
\hline$a$ & $0.4691 \mathrm{~m}$ & Horizontal coordinate position of free end with respect of fixed frame \\
\hline$b$ & $0.15565 \mathrm{~m}$ & Vertical coordinate position of free end with respect of fixed frame \\
\hline$c$ & $-0.024505 \mathrm{~m}$ & Out of plane coordinate position of free end with respect of fixed frame \\
\hline$h$ & $0.005 \mathrm{~m}$ & Height of the beam \\
\hline$w$ & $0.01 \mathrm{~m}$ & Width of the beam \\
\hline$\alpha$ & $120^{\circ}$ & Force angle in the yz-plane \\
\hline$\beta$ & $90^{\circ}$ & Force angle in the xy-plane \\
\hline$L$ & $0.5 \mathrm{~m}$ & Length of the beam \\
\hline
\end{tabular}

In order to calculate the magnitude of the force applied by an actuator along the inclined slider, the $3 \mathrm{D}$ PRBM is used to generate a system of equations. The unknowns are $\Phi, \gamma, \Theta, \Gamma, \Upsilon,{ }^{\{D\}} F_{z}$ and ${ }^{\{D\}} F_{y}$. The material of the beam is steel with a Young's modulus of $200 \mathrm{GPa}$. Using the nominal value of $K_{\Theta}=2.513251$ for AR of 0.5 , the system of equations is solved. The first three equations can be solved independently to obtain $\Phi, \gamma$ and $\Theta$. Then, the non-linear system of equations (138-139) is solved to obtain $\Gamma$ and $\Upsilon$. Finally, we solve the non-linear system of equations (140-141) to obtain ${ }^{\{D\}} F_{z}$ and ${ }^{\{D\}} F_{y}$.

$$
\begin{gathered}
\Phi=\tan ^{-1}\left(\frac{c}{b}\right) \\
\gamma=\frac{\left(\frac{b}{l}\right)^{2}+\left(\frac{c}{l}\right)^{2}+\left(1-\frac{a}{l}\right)^{2}}{2\left(1-\frac{a}{l}\right)^{2}} \\
\Theta=\tan ^{-1}\left(\frac{\frac{b}{l \cos \Phi}}{\left(\frac{a}{l}-1\right)+\gamma}\right)
\end{gathered}
$$




$$
\begin{gathered}
\left(\frac{{ }^{\{C\}} I_{x x} G}{(1-\gamma) L} \gamma-\gamma K_{\Theta} \frac{E}{L} I_{R} \Theta^{2} \sin (2 \Gamma)\right)=0 \\
\Phi=\Upsilon+\Gamma \\
\left(\gamma L^{\{D\}} F_{y}-\gamma K_{\Theta} \frac{E}{L}\left(\bar{I}-I_{R} \cos (2 \Gamma)\right) \Theta\right)=0 \\
\left(\gamma L^{\{D\}} F_{z} \sin \Theta-\frac{{ }^{\{C\}} I_{x x} G}{(1-\gamma) L} \gamma\right)=0
\end{gathered}
$$

Once the $\mathrm{y}$ and $\mathrm{z}$ force components in the $\{\mathrm{D}\}$ frame are determined, the equation of force mapping of frames $\{A\}$ and $\{D\}$ is used to calculate the force magnitude applied by the linear actuator. This process can be repeated with the predicted value of $K_{\Theta}=f(\alpha, \beta, \tilde{s}, A R)$ using the regression for AR 0.5 to obtain a more refined estimate of the force magnitude.

$$
{ }^{\{D\}} \vec{F}=R_{Z}{ }^{T}(\Theta) R_{X}{ }^{T}(\Phi)\left[\begin{array}{c}
\|F\| \sin \alpha \cos \beta \\
\|F\| \sin \alpha \sin \beta \\
\|F\| \cos \alpha
\end{array}\right]
$$

Using the z-component of the previous equation:

$$
{ }^{\{D\}} F_{z}=\|F\| \cos \alpha \cos \Phi-\|F\| \sin \alpha \sin \beta \sin \Phi
$$

Results are presented in the Table 6. 
Table 6 Results from compliant spatial double slider

\begin{tabular}{|c|l|c|l|}
\hline Parameter & Value & Parameter & Value \\
\hline$\Phi$ & $-8.946^{\circ}$ & $\gamma$ & $-0.6635^{\circ}$ \\
\hline$\gamma$ & 0.83468 & ${ }^{\{D\}} F_{y}$ & $86.13 \mathrm{~N}$ \\
\hline$\Theta$ & $22.182^{\circ}$ & ${ }^{\{D\}} F_{z}$ & $-35.62 \mathrm{~N}$ \\
\hline$\Gamma$ & & $\|F\|$ & $99.16 \mathrm{~N}$ \\
\hline
\end{tabular}

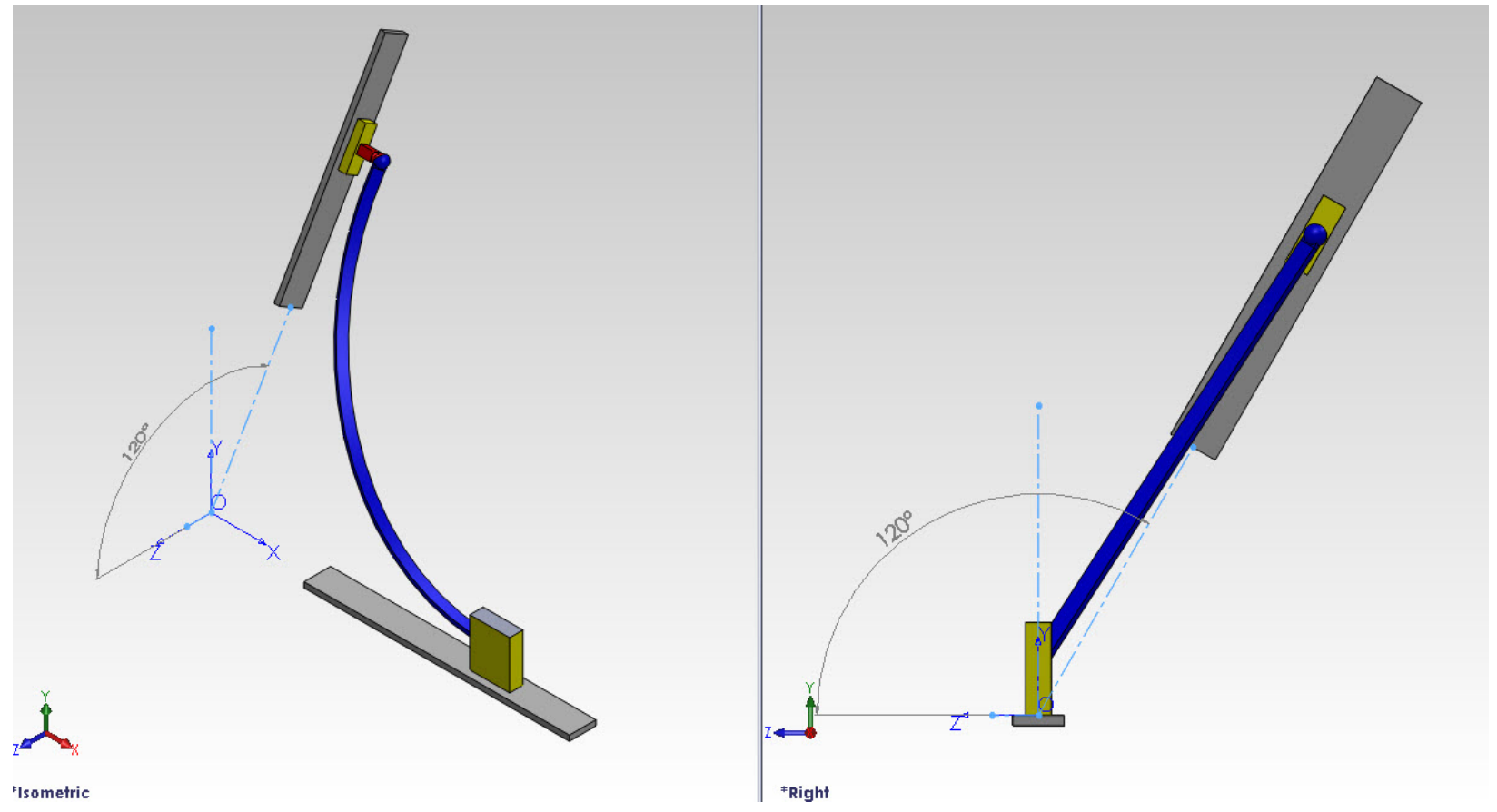

Figure 66 Double slider mechanisms. Left: Isometric view. Right: Front view 


\section{CHAPTER 8: CONCLUSIONS}

The first three-dimensional pseudo-rigid-body model (3-D PRBM) for straight cantilever beams with rectangular cross section was presented. The model is capable of capturing the behavior of the neutral axis of a beam loaded with an arbitrary force end-load. Using the 3-D PRBM designers can predict out of plane bending of spatial beams used in compliant mechanisms.

The data set used to develop the PRBM parameters was obtained solving a system of differential equations that describe the neutral axis of an inextensible beam and the rotation of the rectangular cross section about the neutral axis.

The PRBM approximates the relative position and orientation of the ends of a compliant beam. Model parameters have been obtained for several aspect ratios and the results have been verified using ANSYS. The model's use in design has been illustrated for two spatial compliant mechanisms.

There are several features of this PRBM which are novel, and are unique contributions of this dissertation. Specifically, the kinematic arrangement of the beam is new. Planar PRBM's use revolute joints whereas the spatial PRBM uses spherical joints. More importantly, the stiffness of the $1^{\text {st }}$ spherical joint has a number of novel ideas. The joint decomposes the motion of the beam tip into three different effects including beam bending and two different out of plane motion effects. The first out of plane effect is the result of the torsion in the beam and is related to the torsional stiffness term $\frac{I_{x x} G}{(1-\gamma) L} \Upsilon$. The second out of plane effect is due to tensorial stiffness of the rectangular beam, and results in smaller deflections when the beam is wider. This effect is associated with the term $\gamma K_{\Theta} \frac{E}{I} \Theta^{2} I_{r} \sin (2 \Gamma)$. The quadratic dependence on $\Theta$ is predicted by virtual work but has not been previously published in literature. These effects vanish in the planar and axisymmetric beam cases which have been discussed in previous work. 
It is hoped that future work using this 3D PRBM will yield design insights that will improve the design of compliant mechanisms. 


\section{REFERENCES}

[1] Howell, L. L., 2001, "Compliant Mechanisms," Wiley, New York.

[2] Hoetmer, Karin, Herder, Just L and Kim, Charles. “A Building Block Approach for the Design of Statically Balanced Compliant Mechanisms”. International Design Engineering Technical Conference San Diego, California, USA,2009. Vols. DETC2009 87451.

[3] Jensen, B. D., and Howell, L. L., 2004, "Bistable Configurations of Compliant Mechanisms Modeled using Four Links and Translational Joints," Journal of Mechanical Design, University of Michigan, Ann Arbor, MI, pp. 657-665.

[4] Howell, L. L. and Midha, A. Parametric Deflection Approximations for End-Loaded, LargeDeflection Beams in Compliant Mechanisms.. 1, s.l. : ASME, March 1995, Journal of Mechanical Design, Vol. 117, pp. 156-165.

[5] Howell, L.L. and Midha, A., 1995b, "Determination of the Degrees of Freedom of Compliant Mechanisms Using the Pseudo-Rigid-Body Model Concept,” Proceedings of the Ninth World Congress on the Theory of Machines and Mechanisms, Milano, Italy, Vol. 2, pp. 1537-1541.

[6] Howell, L.L. and Midha, A., 1994b, “The Development of Force-Deflection Relationships for Compliant Mechanisms,” Machine Elements and Machine Dynamics, DE-Vol. 71, 23rd ASME Biennial Mechanisms Conference, pp. 501-508.

[7] Howell, L.L. and Midha, A., 1995b, "Determination of the Degrees of Freedom of Compliant Mechanisms Using the Pseudo-Rigid-Body Model Concept,” Proceedings of the Ninth World Congress on the Theory of Machines and Mechanisms, Milano, Italy, Vol. 2, pp. 1537-1541.

[8] Saxena, A. and Kramer, S.N., 1998, “A Simple and Accurate Method for Determining Large Deflections in Compliant Mechanisms Subjected to End Forces and Moments,” Journal of Mechanical Design, Trans. ASME, Vol. 120, No.3, pp. 392-400, erratum, Vol. 121, No. 2, p.194.

[9] Dado M. H, 2001 "Variable Parametric Pseudo-Rigid Body Model for Large Deflection Beams with End Loads” International Journal of Non-linear Mechanics, 2001.

[10] Nielson, A.J. and Howell, L.L., 1998, “Compliant Pantographs via the Pseudo-Rigid-Body Model,” Proceedings of the 1998 ASME Design Engineering Technical Conferences, DETC98/MECH-5930. 
[11] Crane, N.B., Howell L.L., and Weight, B. L., 2000, "Design and Testing of a Compliant FloatingOpposing Arm (FOA) Centrifugal Clutch,” Proceedings of 8th International Power Transmission and Gearing Conference, 2000 ASME Design Engineering Technical Conferences DETC2000/PTG-14451.

[12] Roach, G.M., and Howell, L. L., 2000, “Compliant Overrunning clutch with centrifugal Throw out,” U.S. patent 6,148,979, Nov 21.

[13] Baker, M.S., Lyon, S.M., and Howell L.L., 2000, “A Linear Displacement Bistable Micromechanism,” Proceedings of the 26th Biennial Mechanisms and Robotics Conference, 2000 ASME Design Engineering Technical Conferences, DETC2000/MECH- 14119.

[14] C. Lusk and L. Howell, \A Micro Helico-Kinematic Platform (MHKP) via spherical cranksliders," Proceedings of the International Mechanical Engineering Conference and Exposition (IMECE), Anaheim CA, November 13-19, 2001.

[15] N.B. Hubbard, L.L. Howell, 2005 "Design and Characterization of a Duel-stage, Thermally Actuated Nanopositioner”, Journal of Micromechanics and Microengineering, 2005.

[16] Jensen, B.D., Howell L.L., and Salmon, L.G., 1999, "Design of Two-Link, In-plane, Bi-stable Compliant Micro-mechanisms,” Journal of Mechanical Design, Trans. ASME, Vol. 121, No. 3, pp.93-96.

[17] Yue-Qing Yu, Zhong-Lei Feng, Qi-Ping Xu, A pseudo-rigid-body 2R model of flexural beam in compliant mechanisms, Mechanism and Machine Theory, Volume 55, September 2012, Pages 18-33, ISSN 0094-114X, http://dx.doi.org/10.1016/j.mechmachtheory.2012.04.005. (http://www.sciencedirect.com/science/article/pii/S0094114X12000948)

[18] Su, H., 2009. “A Pseudo-rigid-body 3R model for determining large-deflection of cantilever beams subject to tip loads”. J. Mechanisms Robotics 1 (2): 021008

[19] Halverson, PA, AE Bowden, and LL Howell. "A Pseudo-Rigid-Body Model Of The Human Spine To Predict Implant-Induced Changes On Motion." Journal Of Mechanisms And RoboticsTransactions Of The Asme 3.4 (n.d.)

[20] Peter A., Halverson, Bowden Anton E., and Howell Larry L. "A Compliant-Mechanism Approach To Achieving Specific Quality Of Motion In A Lumbar Total Disc Replacement." The International Journal Of Spine Surgery 6.(n.d.): 78-86.

[21] Craig P., Lusk, and Howell Larry L. "Spherical Bistable Micromechanism." Journal Of Mechanical Design 130.4 (2008): 45001

[22] Sonmez, U, and CC Tutum. "A Compliant Bistable Mechanism Design Incorporating Elastica Buckling Beam Theory And Pseudo-Rigid-Body Model." Journal Of Mechanical Design 130.4 (n.d.) 
[23] John A., Kennedy, Howell Larry L., and Greenwood William. "Compliant High-Precision EQuintet Ratcheting (CHEQR) Mechanism For Safety And Arming Devices." Precision Engineering 31.(n.d.): 13-21.

[24] Chase, Robert P., et al. "A 3-D Chain Algorithm With Pseudo-Rigid-Body Model Elements." Mechanics Based Design Of Structures \& Machines 39.1 (2011): 142-156.

[25] Umesh, Bhagat, et al. "Design And Analysis Of A Novel Flexure-Based 3-DOF Mechanism." Mechanism And Machine Theory 74.(n.d.): 173-187.

[26] Kern, Dominik, et al. "Key Features Of Flexure Hinges Used As Rotational Joints: Transition From Concentrated To Distributed Compliances." Forschung Im Ingenieurwesen 77.3/4 (2013): 117.

[27] Morgan D., Murphy, Midha Ashok, and Howell Larry L. "The Topological Synthesis Of Compliant Mechanisms." Mechanism And Machine Theory 31.(n.d.): 185-199

[28] Ashok, Midha, Howell Larry L, and Norton Tony W. "Limit Positions Of Compliant Mechanisms Using The Pseudo-Rigid-Body Model Concept." Mechanism And Machine Theory 35.(n.d.): 99115

[29] Wang, NF, XH Liang, and XM Zhang. "Pseudo-Rigid-Body Model For Corrugated Cantilever Beam Used In Compliant Mechanisms." Chinese Journal Of Mechanical Engineering 27.1 (n.d.): 122-129.

[30] Lusk, C. “Quantifying uncertainty for planar pseudo-rigid-body models”, Proceedings of the ASME 2011 International Design Engineering Technical Conferences\& Computers and Information in Engineering Conference. DETC2011-47456

[31] Frisch-Fay, R. 1962. Flexible bars. Washington, D.C.: Butterworths.

[32] Ramirez, I., Lusk, C. "Spatial beam large deflection equations and pseudo-rigid-body model for axisymmetric cantilever beams”, Proceedings of the ASME 2011 International Design Engineering Technical Conferences\& Computers and Information in Engineering Confernece. DETC2011-47389

[33] Volkan Parlaktaş, Engin Tanık, Partially compliant spatial slider-crank (RSSP) mechanism, Mechanism and Machine Theory, Volume 46, Issue 11, November 2011, Pages 1707-1718, ISSN 0094-114X, http://dx.doi.org/10.1016/j.mechmachtheory.2011.06.010. 
APPENDICES 


\section{Appendix A Nomenclature}

$(\psi, \theta, \phi) \quad$ Euler angles in the XZX angle rotation set for spatial beam

$\tau_{x} \quad$ Curvature about x-axis

$\kappa_{y} \quad$ Curvature about y-axis

$\kappa_{z} \quad$ Curvature about z-axis

$\mathrm{X}, \mathrm{Y}, \mathrm{Z} \quad$ Relative position coordinates from beam ends

\{A $\quad$ Fixed end of the beam

$\{\mathrm{H}\} \quad$ Free end of the beam

$\mathrm{F}$

Force applied at the free end of the beam

$\{S\} R \quad$ Describes the orientation of frame $\{\mathrm{S}\}$ with respect to $\{\mathrm{Q}\}$

$(\eta, \xi) \quad$ Angles of a spherical coordinate system to define force orientation

E Young's modulus

G Modulus of rigidity

I Second moment of area

${ }^{\{Q\}} \mathcal{V} \quad$ Vector defined in the frame $\{\mathrm{Q}\}$

$\mathrm{S}$

Arc length

$\tilde{v} \quad$ Quantities with tilde are dimensionless

(a,b,c) Position coordinates from fixed end to free end in $\mathrm{x}-, \mathrm{xy}-$ and $\mathrm{z}$ - components

$\gamma \quad$ Characteristic radius factor

$\Theta \quad$ Bending pseudo-rigid-body angle

$\Phi \quad$ Twisting pseudo-rigid-body angle

$(\Sigma, \Omega, \Psi) \quad$ Euler angles in the XZX angle rotation set for the second spherical joint

$\left(c_{\phi}, c_{\theta}, c_{\psi}\right) \quad$ Parametric angle coefficients

$V_{T} \quad$ Torsion potential energy

$V_{B} \quad$ Bending potential energy 


\section{Appendix B Regressions and Statistical Values for PRBM Parameters}

PRBM constants parameter regressions and statistical analysis are presented in Tables A-H where AR is height divided by width of rectangular cross-section and $\mathrm{N}$ is number of points.

Table A Coefficient vector for parameter fits with $\mathrm{AR}=1$

\begin{tabular}{|r|r|r|}
\hline \multicolumn{3}{|c|}{$\mathrm{AR}=1$} \\
\hline $\overrightarrow{B_{c_{\theta}}}$ & $\overrightarrow{B_{K_{\theta}}}$ & \multicolumn{1}{|c|}{$\overrightarrow{B_{\gamma}}$} \\
\hline 1.245999 & 2.550507 & 0.835116 \\
\hline 0.001713 & -0.0054 & -0.0015 \\
\hline 0.002588 & 0.016341 & -0.00335 \\
\hline 0.000492 & -0.01413 & 0.000105 \\
\hline$-2.26 \mathrm{E}-05$ & -0.00095 & $6.35 \mathrm{E}-05$ \\
\hline 0.026739 & -0.13772 & -0.02076 \\
\hline-0.01045 & 0.094145 & 0.006174 \\
\hline-0.00014 & 0.022755 & -0.00085 \\
\hline-0.00356 & 0.026582 & 0.002473 \\
\hline-0.01142 & 0.002288 & 0.011436 \\
\hline $\mathrm{R}^{2}$ & $\mathrm{R}^{2}$ & $\mathrm{R}^{2}$ \\
\hline 0.9762 & 0.9716 & 0.9767 \\
\hline
\end{tabular}

Table B Coefficient vector for parameter fits with $\mathrm{AR}=0.8$

\begin{tabular}{|c|r|r|r|r|r|}
\hline \multicolumn{7}{|c|}{$\mathrm{AR}=0.8$} \\
\hline $\overrightarrow{B_{c_{\psi}}}$ & $\overrightarrow{B_{c_{\phi}}}$ & $\overrightarrow{B_{c_{\theta}}}$ & $\overrightarrow{B_{K_{\theta}}}$ & $\overrightarrow{B_{\gamma}}$ & $\overrightarrow{B_{c_{\psi / \Phi}}}$ \\
\hline 1.008355 & 1.0199 & 1.242824 & 2.566706 & 0.837714 & -0.86756 \\
\hline 0.000689 & 0.001958 & 0.000575 & 0.001684 & -0.00056 & 0.005649 \\
\hline 0.000954 & 0.002617 & 0.00211 & 0.005865 & -0.00238 & 0.031939 \\
\hline-0.00667 & -0.01161 & 0.002228 & -0.03174 & -0.00097 & -0.09202 \\
\hline-0.00529 & -0.0084 & 0.002468 & -0.01959 & -0.00165 & -0.10064 \\
\hline-0.01335 & -0.03221 & 0.035084 & -0.17302 & -0.02732 & -0.07542 \\
\hline 0.005251 & 0.016781 & -0.01365 & 0.111731 & 0.008344 & 0.07655 \\
\hline 0.001661 & 0.002792 & $-9.14 \mathrm{E}-06$ & 0.007144 & -0.00025 & 0.012466 \\
\hline 0.015721 & 0.022817 & -0.00677 & 0.063974 & 0.004029 & 0.174033 \\
\hline 0.001873 & -0.00095 & -0.01147 & 0.006116 & 0.011314 & -0.0732 \\
\hline & & & & & -0.0277 \\
\hline $\mathrm{R}^{2}$ & $\mathrm{R}^{2}$ & $\mathrm{R}^{2}$ & $\mathrm{R}^{2}$ & $\mathrm{R}^{2}$ & $\mathrm{R}^{2}$ \\
\hline 0.9243 & 0.9173 & 0.9785 & 0.977 & 0.9725 & 0.9122 \\
\hline
\end{tabular}


Table C Coefficient vector for parameter fits with AR=0.5

\begin{tabular}{|c|r|r|r|r|c|}
\hline \multicolumn{7}{|c|}{$\mathrm{AR}=0.5$} \\
\hline $\overrightarrow{B_{c_{\psi}}}$ & $\overrightarrow{B_{c_{\phi}}}$ & $\overrightarrow{B_{c_{\theta}}}$ & $\overrightarrow{B_{K_{\theta}}}$ & $\overrightarrow{B_{\gamma}}$ & $\overrightarrow{B_{c_{\psi} / \Phi}}$ \\
\hline 1.040541 & 1.053027 & 1.23712 & 2.579304 & 0.843234 & -0.72585 \\
\hline 0.007321 & 0.009098 & -0.00153 & 0.004591 & 0.001599 & 0.007905 \\
\hline 0.004741 & 0.007709 & 0.00179 & 0.008775 & -0.00212 & 0.072041 \\
\hline-0.03174 & -0.03625 & 0.005659 & -0.03775 & -0.00447 & -0.20718 \\
\hline-0.01923 & -0.0214 & 0.004365 & -0.02744 & -0.00342 & -0.25048 \\
\hline-0.09247 & -0.13132 & 0.076997 & -0.35572 & -0.06017 & -0.18826 \\
\hline 0.059883 & 0.092384 & -0.03301 & 0.263092 & 0.019184 & 0.350746 \\
\hline 0.001525 & 0.006472 & $1.12 \mathrm{E}-05$ & 0.014848 & -0.00048 & 0.053669 \\
\hline 0.076919 & 0.082735 & -0.0131 & 0.103684 & 0.009143 & 0.527036 \\
\hline-0.00553 & -0.01092 & -0.01414 & 0.002035 & 0.014066 & -0.21456 \\
\hline & & & & & -0.07516 \\
\hline $\mathrm{R}^{2}$ & $\mathrm{R}^{2}$ & $\mathrm{R}^{2}$ & $\mathrm{R}^{2}$ & $\mathrm{R}^{2}$ & $\mathrm{R}^{2}$ \\
\hline 0.9262 & 0.923 & 0.9781 & 0.9741 & 0.9709 & 0.9245 \\
\hline
\end{tabular}

Table D Coefficient vector for parameter fits with $\mathrm{AR}=0.2$

\begin{tabular}{|r|r|r|r|r|r|}
\hline \multicolumn{7}{|c|}{$\mathrm{AR}=0.2$} \\
\hline $\overrightarrow{B_{c_{\psi}}}$ & $\overrightarrow{B_{c_{\phi}}}$ & $\overrightarrow{B_{c_{\theta}}}$ & $\overrightarrow{B_{K_{\theta}}}$ & $\overrightarrow{B_{\gamma}}$ & $\overrightarrow{B_{c_{\Psi} \Phi}}$ \\
\hline 1.309146 & 1.062439 & 1.229012 & 2.599026 & 0.848115 & -0.79096 \\
\hline 0.035516 & 0.00602 & -0.00111 & 0.010917 & 0.000653 & -0.03162 \\
\hline 0.093532 & 0.025165 & 0.002263 & 0.006868 & -0.00327 & 0.163591 \\
\hline-0.18577 & -0.06928 & 0.011942 & -0.08636 & -0.00852 & -0.30359 \\
\hline-0.11482 & -0.04227 & 0.009996 & -0.06325 & -0.00723 & -0.43315 \\
\hline-1.62345 & -0.11298 & 0.321669 & -0.76976 & -0.25597 & 2.691355 \\
\hline 1.891187 & 0.398936 & -0.1518 & 0.713689 & 0.082649 & 0.770892 \\
\hline 0.312275 & 0.026524 & 0.026272 & -0.04631 & -0.0282 & 0.491652 \\
\hline 0.47909 & 0.256834 & -0.04031 & 0.292262 & 0.028405 & 1.300664 \\
\hline-0.23096 & -0.08232 & -0.02153 & -0.01243 & 0.023181 & -0.68136 \\
\hline & & & & & -0.15455 \\
\hline $\mathrm{R}^{2}$ & $\mathrm{R}^{2}$ & $\mathrm{R}^{2}$ & $\mathrm{R}^{2}$ & $\mathrm{R}^{2}$ & $\mathrm{R}^{2}$ \\
\hline 0.8559 & 0.9438 & 0.9837 & 0.9603 & 0.9842 & 0.9484 \\
\hline
\end{tabular}

Table E Average and standard deviation of PRBM constants for AR=1

\begin{tabular}{|l|c|c|r|c|l|l|}
\hline \multicolumn{8}{|c|}{$\mathrm{AR}=1, \mathrm{~N}=27404$} \\
\hline & $\gamma$ & $K_{\theta}$ & $c_{\psi}$ & $c_{\theta}$ & $c_{\phi}$ & $c_{\Psi / \Phi}$ \\
\hline Mean & 0.831533 & 2.520213 & 1 & 1.250809 & 1 & -1 \\
\hline Std dev. & 0.004615 & 0.036295 & 0 & 0.005545 & 0 & 0 \\
\hline
\end{tabular}


Table F Average and standard deviation of PRBM constants for AR=0.8

\begin{tabular}{|l|c|r|r|r|r|r|}
\hline \multicolumn{7}{|c|}{$\mathrm{AR}=0.8, \mathrm{~N}=27404$} \\
\hline & $\gamma$ & \multicolumn{1}{|c|}{$K_{\theta}$} & \multicolumn{1}{|c|}{$c_{\psi}$} & \multicolumn{1}{c|}{$c_{\theta}$} & \multicolumn{1}{c|}{$c_{\phi}$} & \multicolumn{1}{c|}{$c_{\Psi / \Phi}$} \\
\hline Mean & 0.832444 & 2.513146 & 1.00132 & 1.250225 & 1.001808 & -0.9754 \\
\hline Std dev. & 0.003992 & 0.029249 & 0.003688 & 0.004929 & 0.00537 & 0.041395 \\
\hline
\end{tabular}

Table G Average and standard deviation of PRBM constants for AR=0.5

\begin{tabular}{|l|r|r|r|r|r|r|}
\hline \multicolumn{7}{|c|}{$\mathrm{AR}=0.5, \mathrm{~N}=27404$} \\
\hline & $\gamma$ & \multicolumn{1}{|c|}{$K_{\theta}$} & \multicolumn{1}{c|}{$c_{\psi}$} & $c_{\theta}$ & $c_{\phi}$ & $c_{\Psi / \Phi}$ \\
\hline Mean & 0.83257 & 2.513251 & 1.006218 & 1.250041 & 1.006583 & -0.93683 \\
\hline Std dev. & 0.004228 & 0.03166 & 0.01476 & 0.005325 & 0.016347 & 0.105167 \\
\hline
\end{tabular}

Table H Average and standard deviation of PRBM constants for $\mathrm{AR}=0.2$

\begin{tabular}{|l|r|r|r|r|r|r|}
\hline \multicolumn{7}{|c|}{$\mathrm{AR}=0.2, \mathrm{~N}=20527$} \\
\hline & $\gamma$ & $K_{\theta}$ & $c_{\psi}$ & \multicolumn{1}{c|}{$c_{\theta}$} & $c_{\phi}$ & \multicolumn{1}{c|}{$c_{\Psi / \Phi}$} \\
\hline Mean & 0.832227 & 2.525501 & 1.032149 & 1.249723 & 1.024043 & -0.82221371 \\
\hline Std dev. & 0.00602 & 0.045842 & 0.063924 & 0.00787 & 0.037838 & 0.20329551 \\
\hline
\end{tabular}


Appendix C 3D PRBM Results

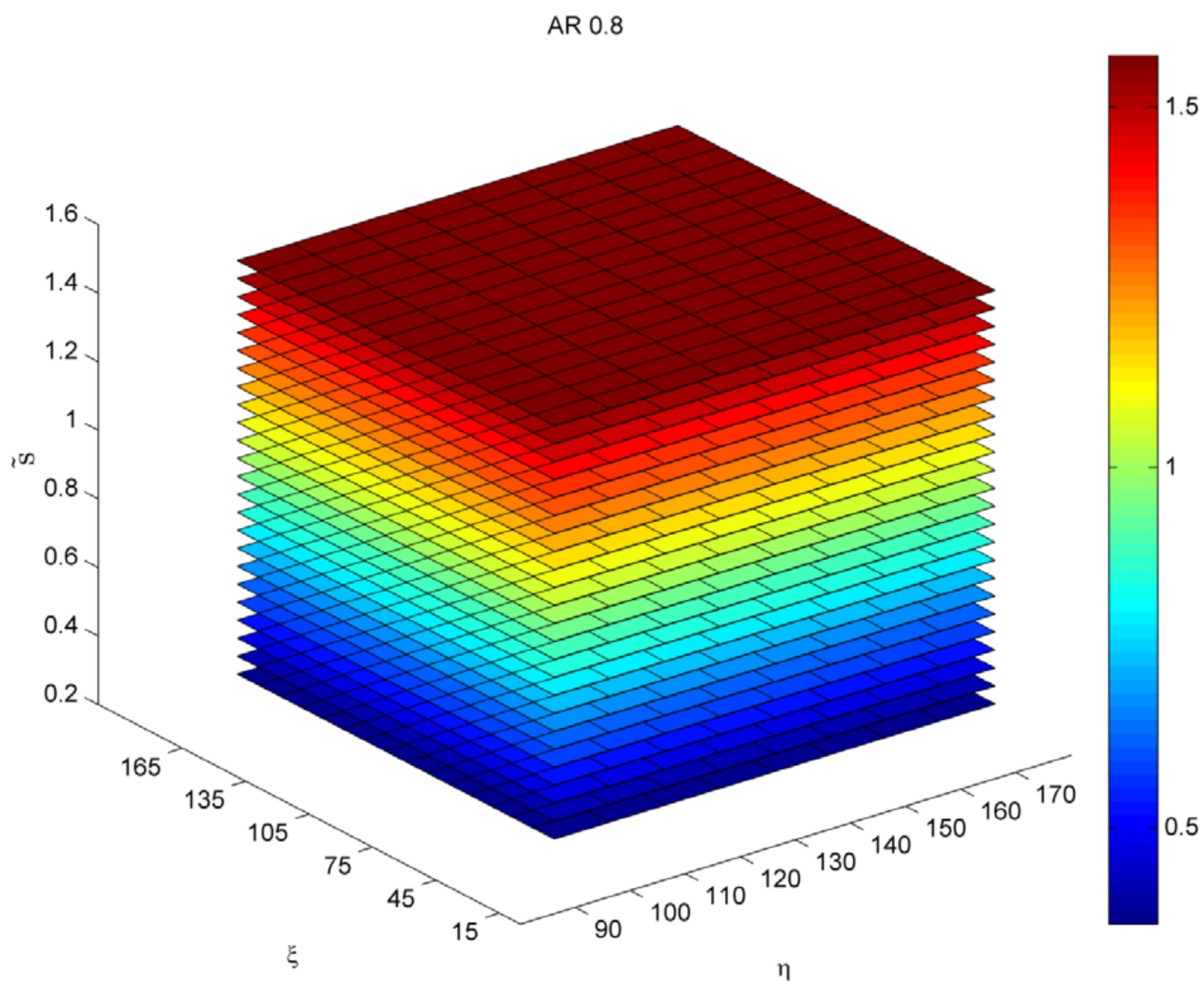

Figure A Nondimensional length for $A R 0.8$ 


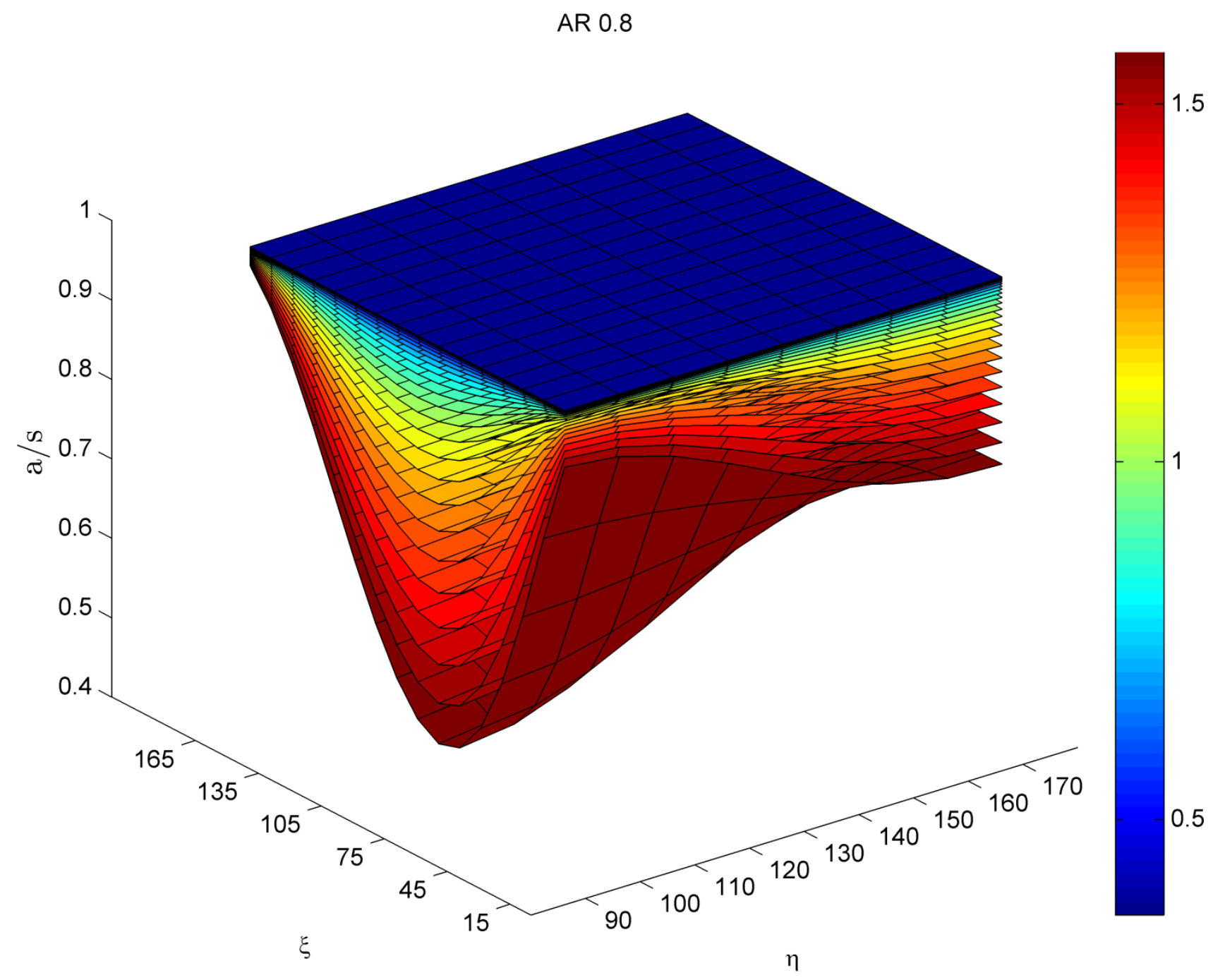

Figure $\mathrm{B}$ a/s for $A R 0.8$ 


\section{AR 0.8}

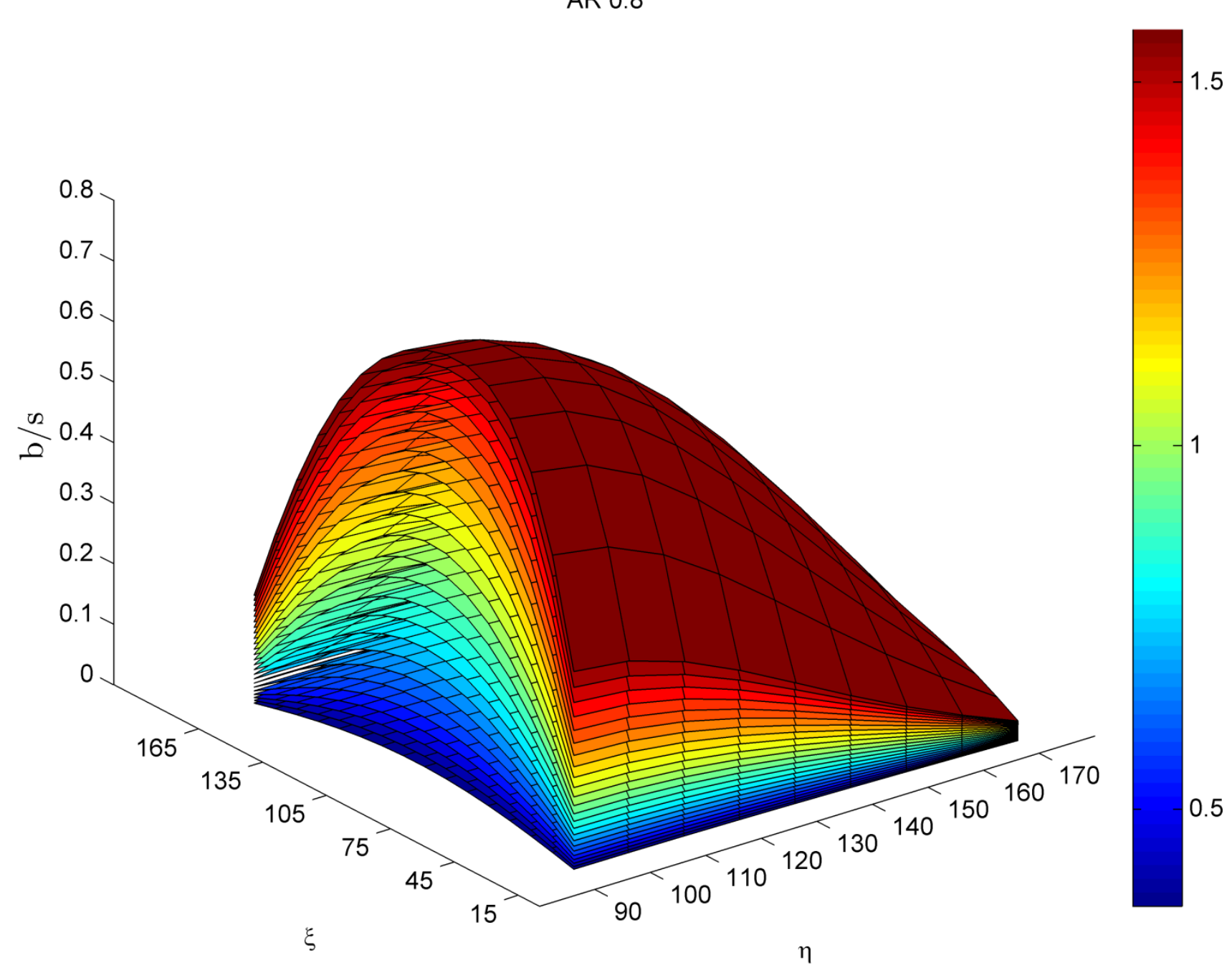

Figure $\mathrm{C} b / \mathrm{s}$ for $A R 0.8$ 


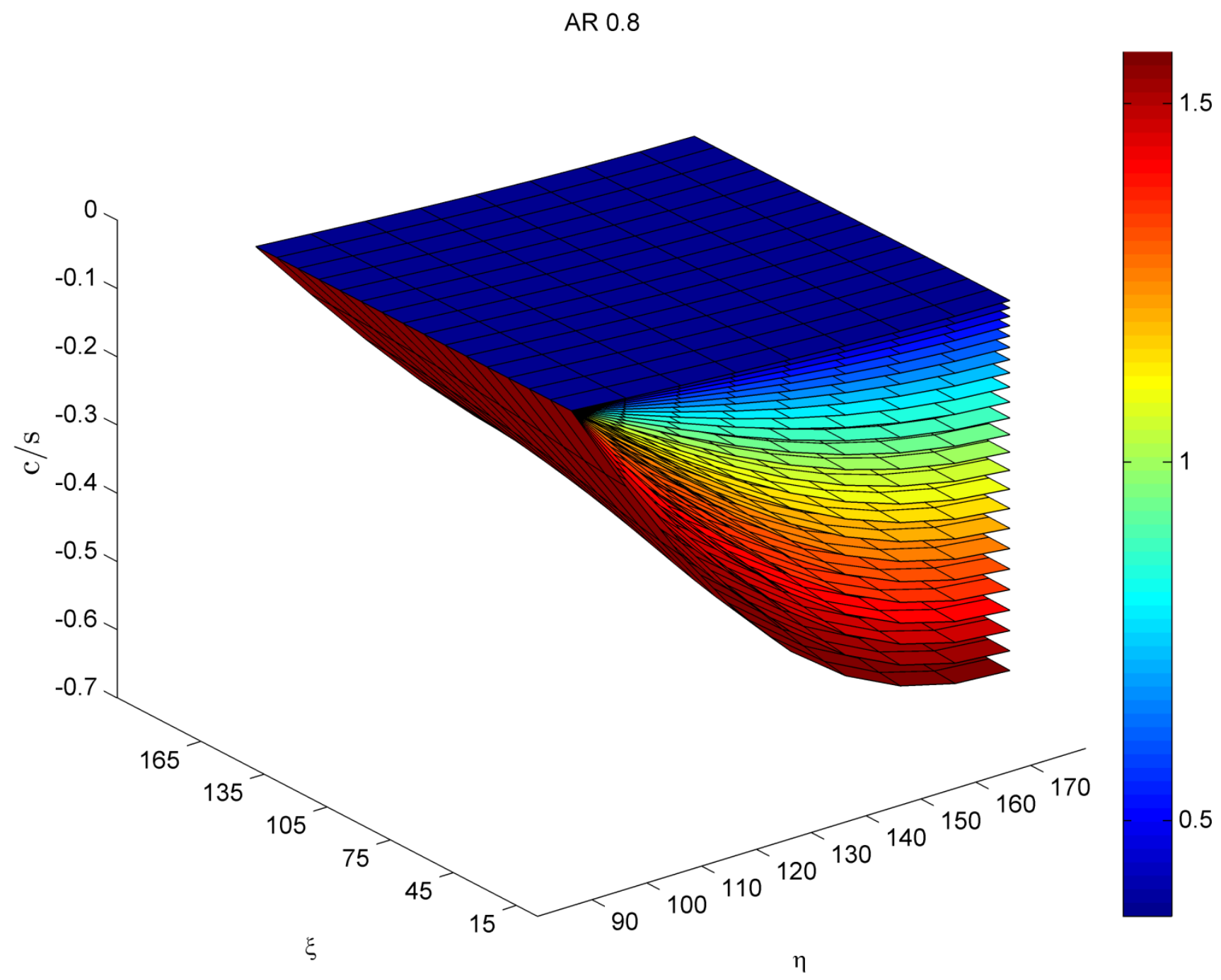

Figure D c/s for $A R 0.8$ 
AR 0.8

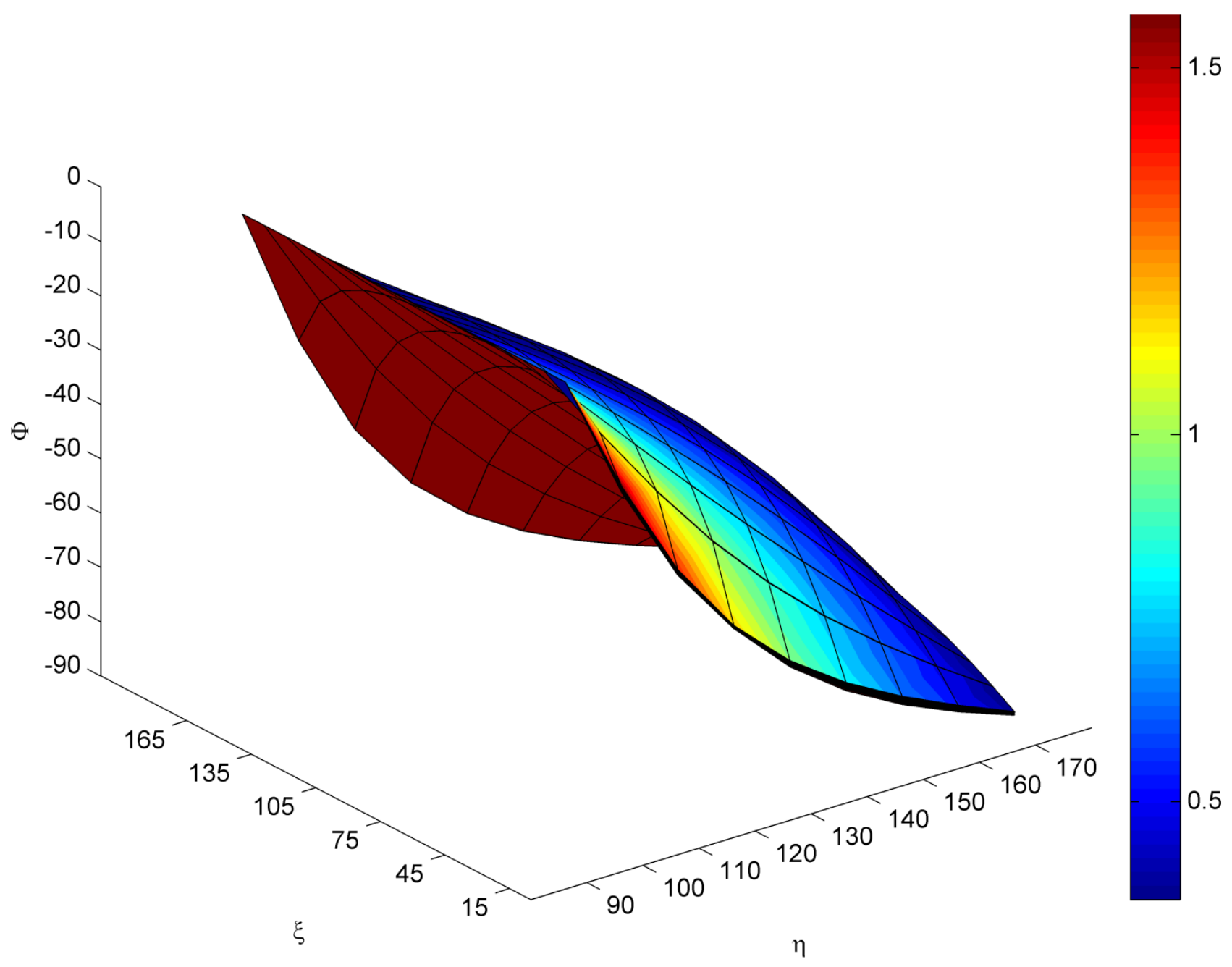

Figure $\mathrm{E} \Phi$ for $A R 0.8$ 


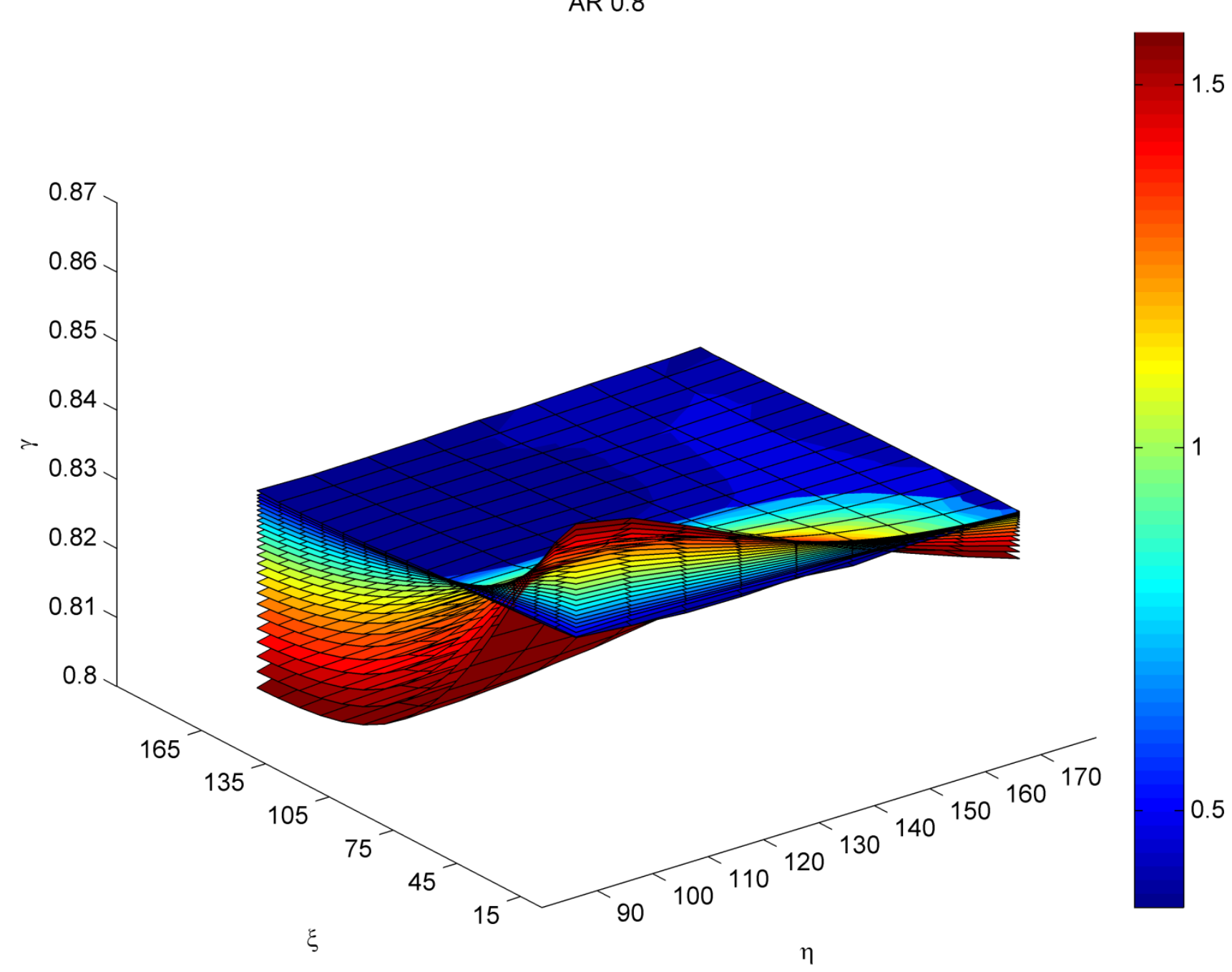

Figure $\mathrm{F} \gamma$ for $A R 0.8$ 
AR 0.8

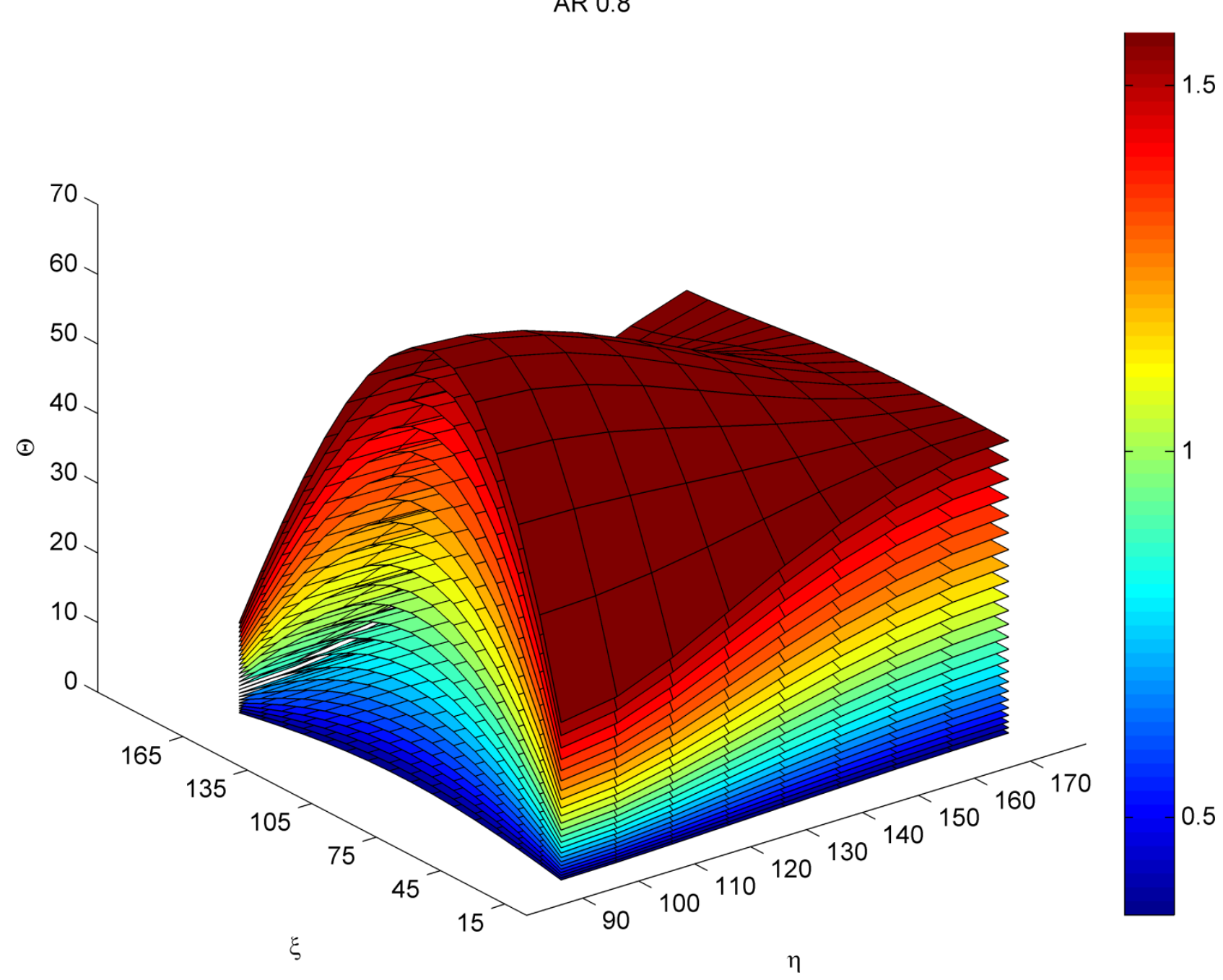

Figure $\mathrm{G} \Theta$ for $A R 0.8$ 


\section{AR 0.8}

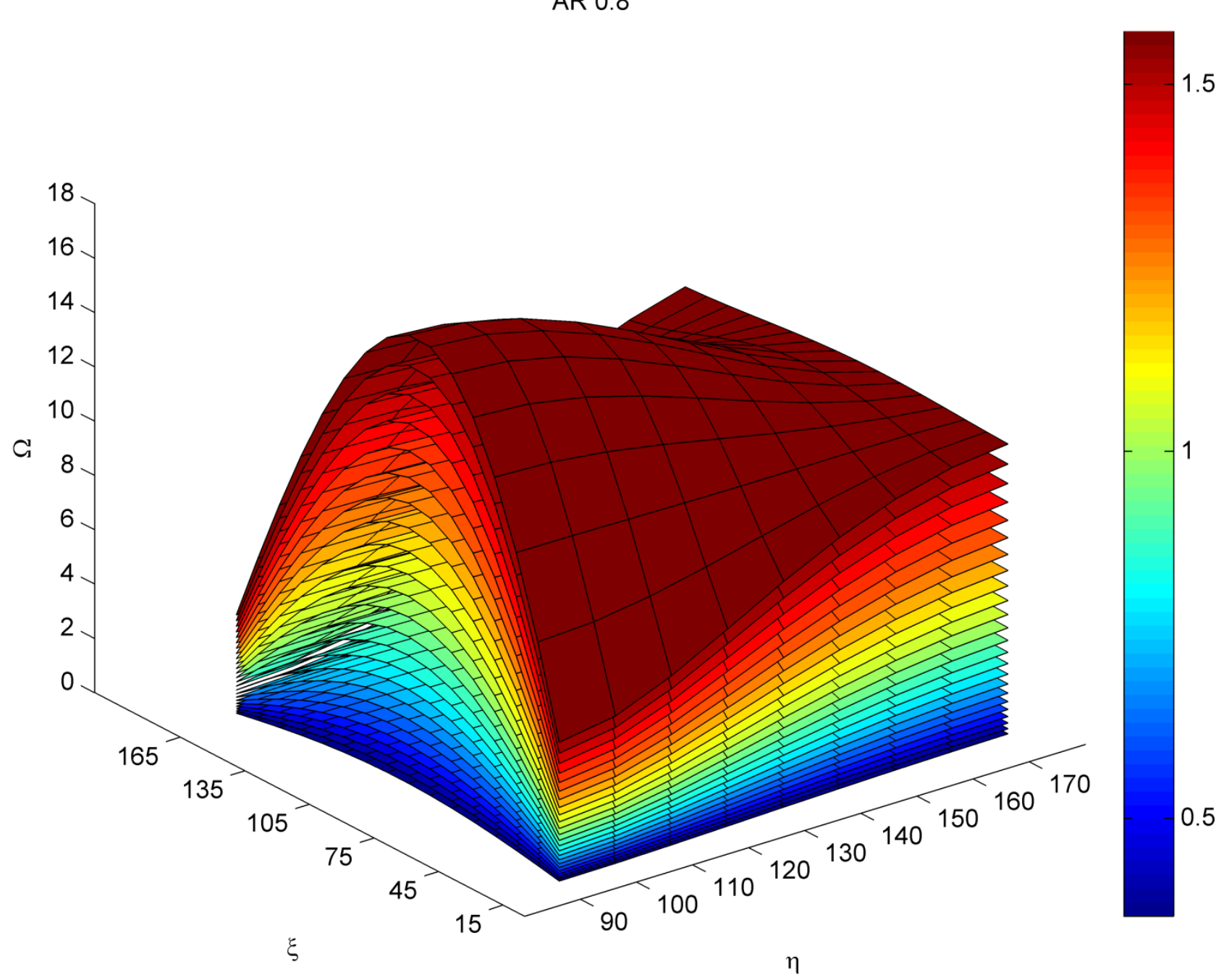

Figure $\mathrm{H} \Omega$ for $A R 0.8$ 
AR 0.8

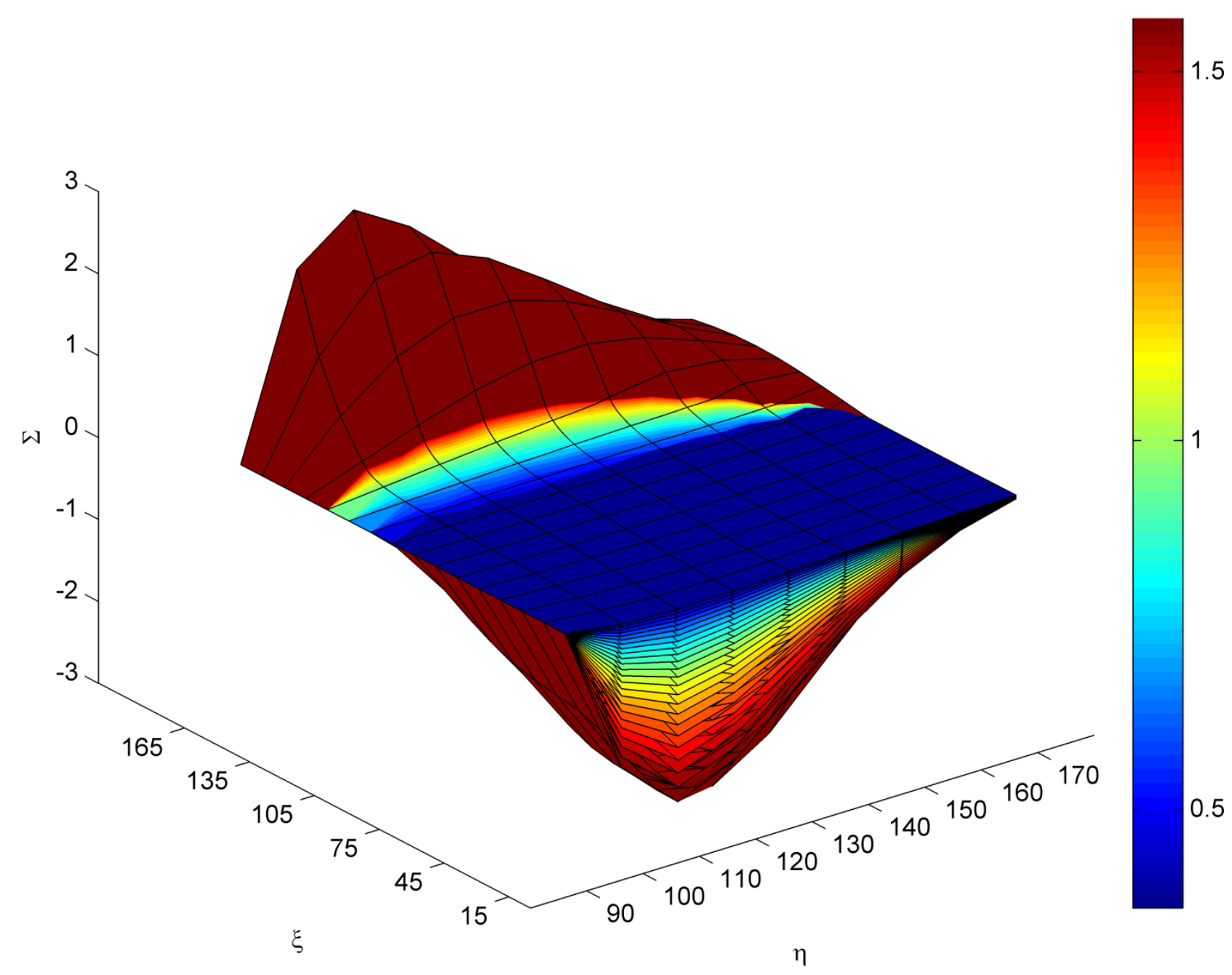

Figure I $\Sigma$ for $A R 0.8$ 


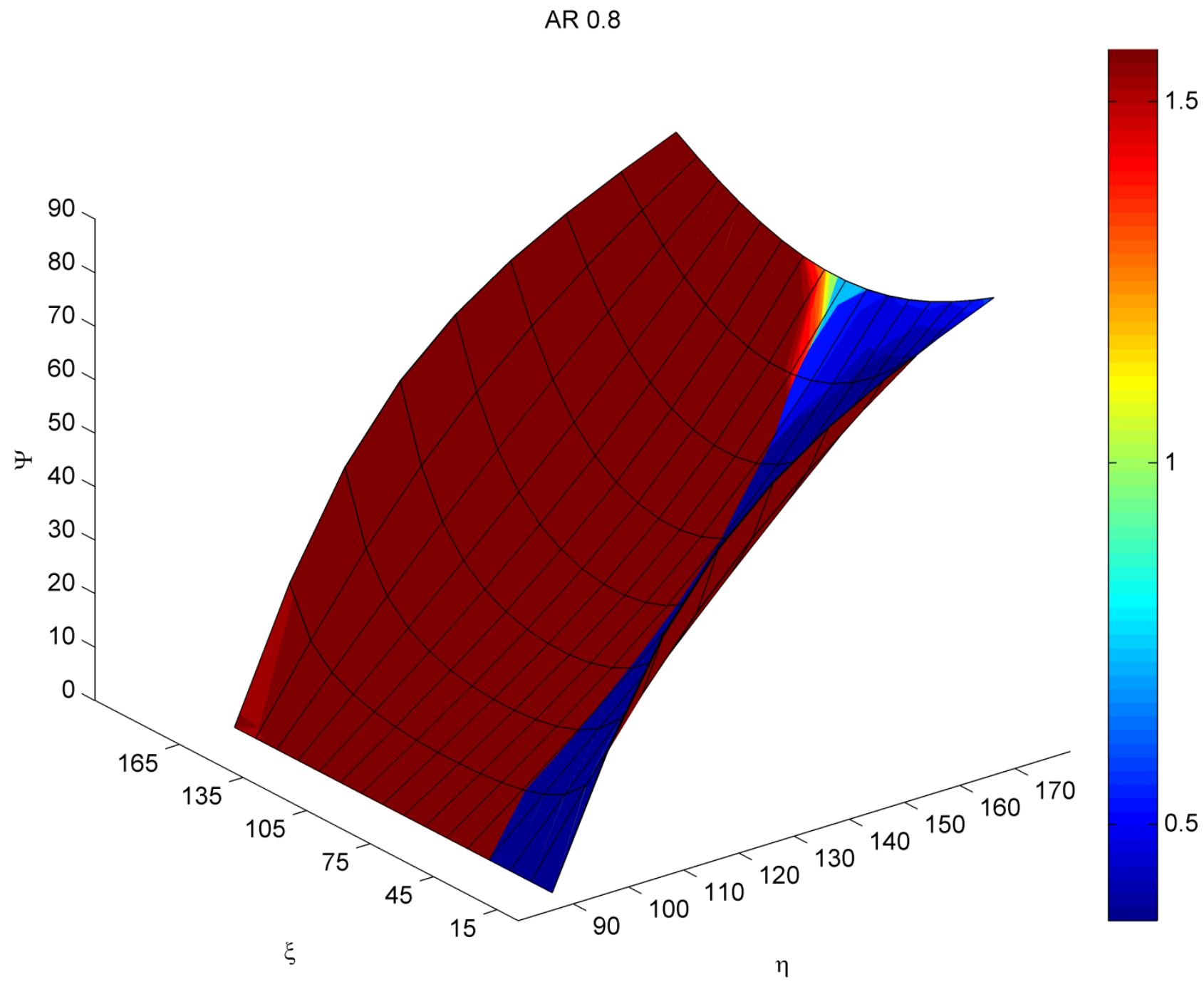

Figure $\mathrm{J} \Psi$ for $A R 0.8$ 


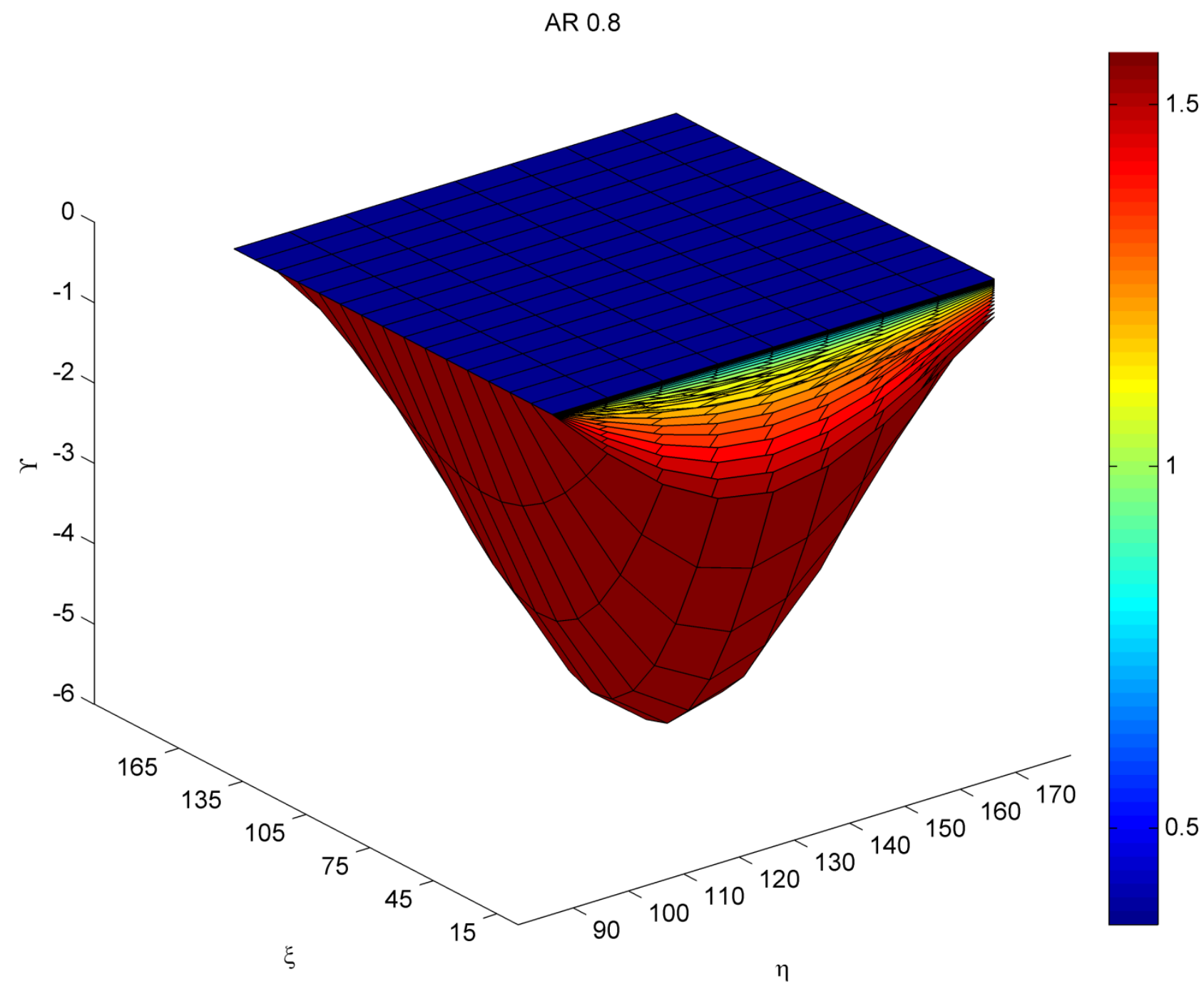

Figure $\mathrm{K} \Upsilon$ for $A R 0.8$ 
AR 0.8

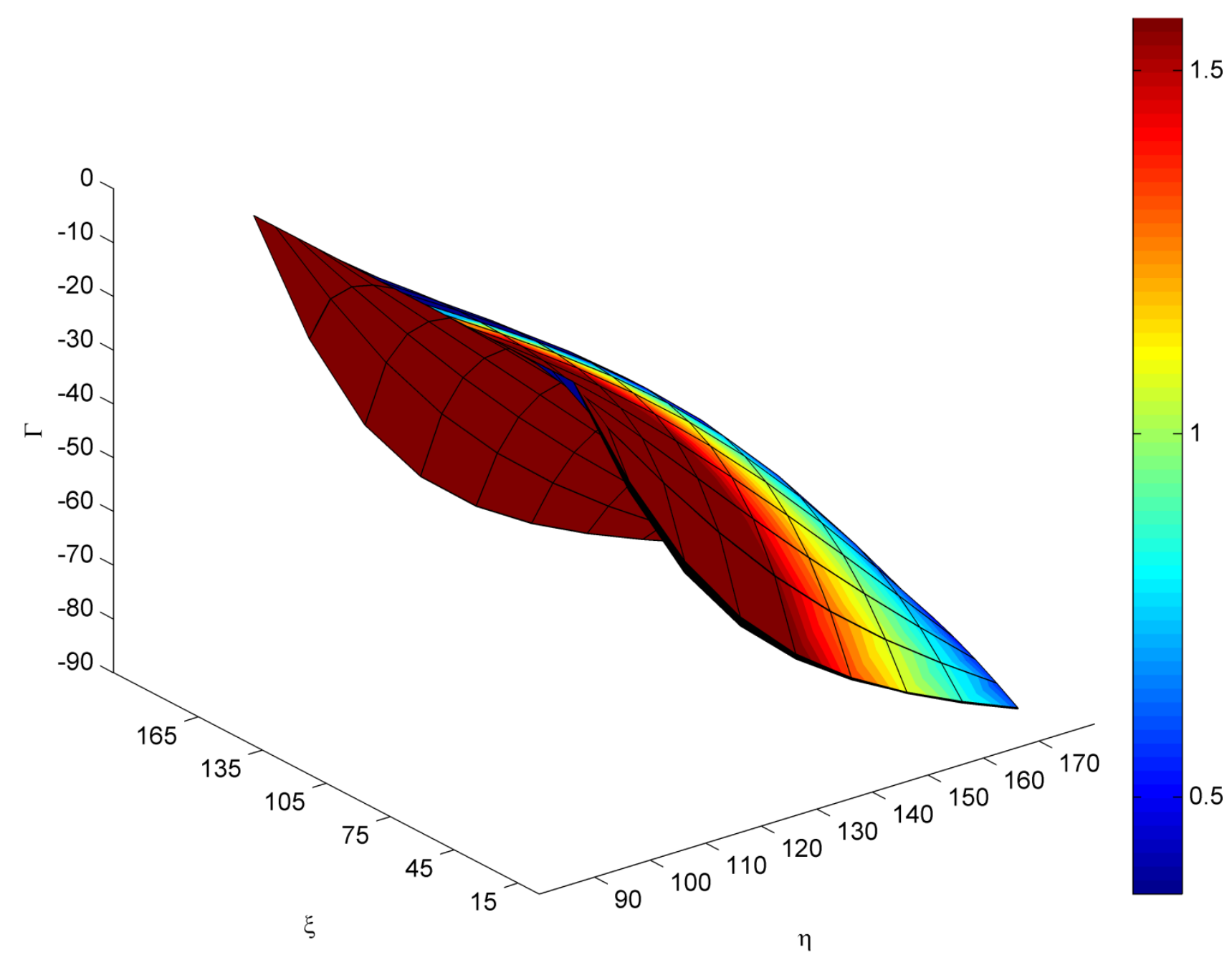

Figure $L \Gamma$ for $A R 0.8$ 


\section{AR 0.8}

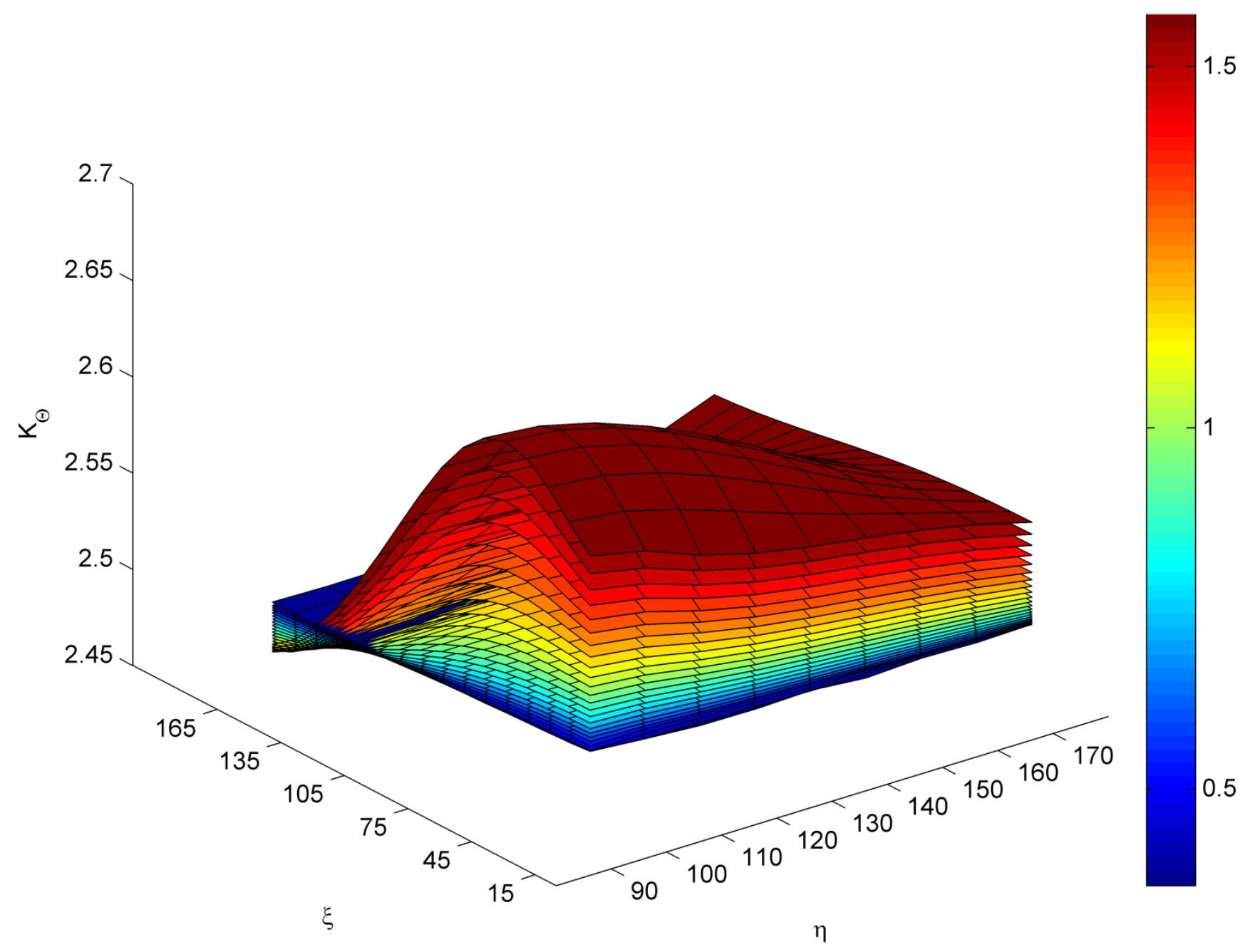

Figure $\mathrm{M} K_{\Theta}$ for AR 0.8 
AR 0.8

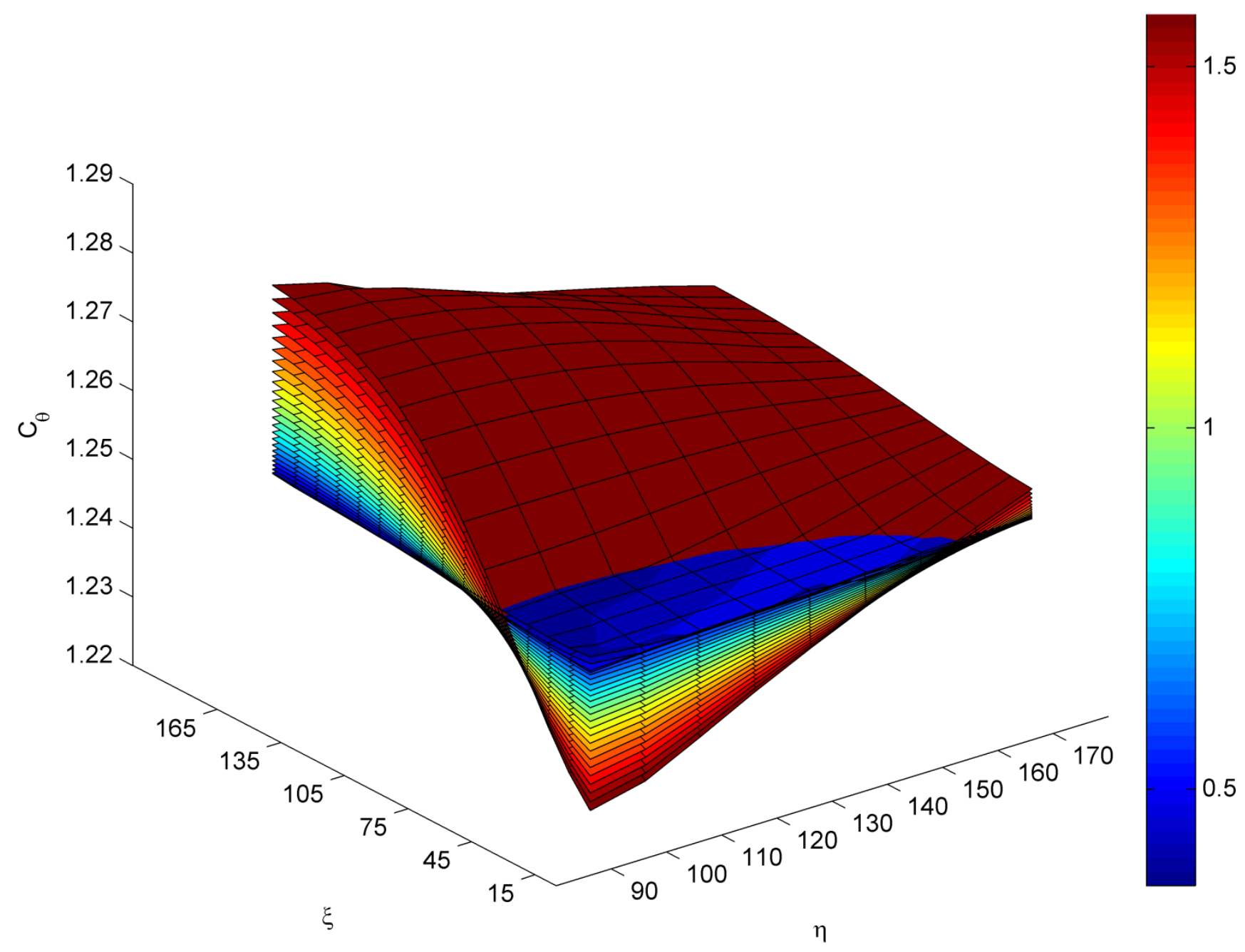

Figure $\mathrm{N} c_{\theta}$ for $A R 0.8$ 
AR 0.8

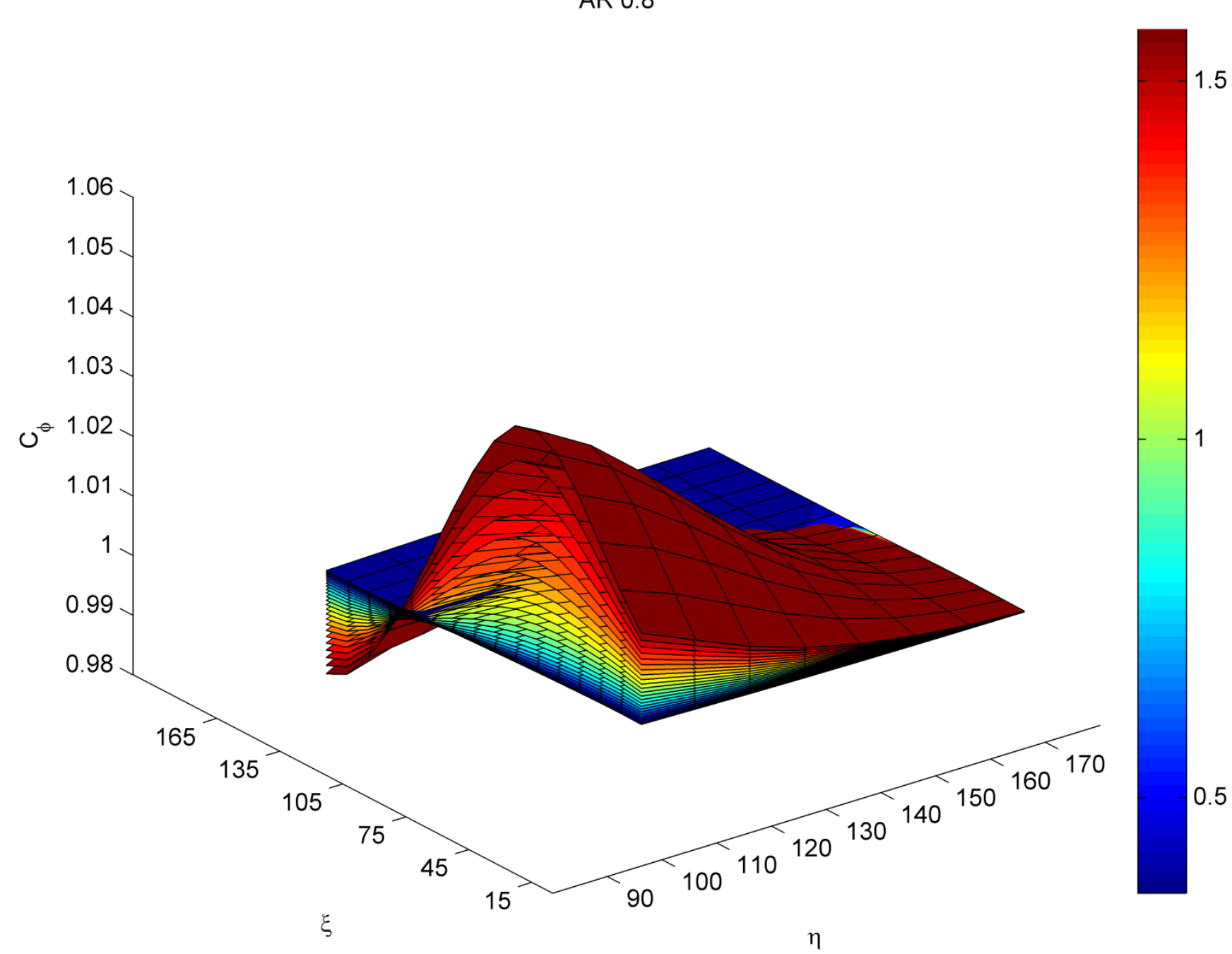

Figure $\mathrm{O} c_{\phi}$ for $A R 0.8$ 
AR 0.8

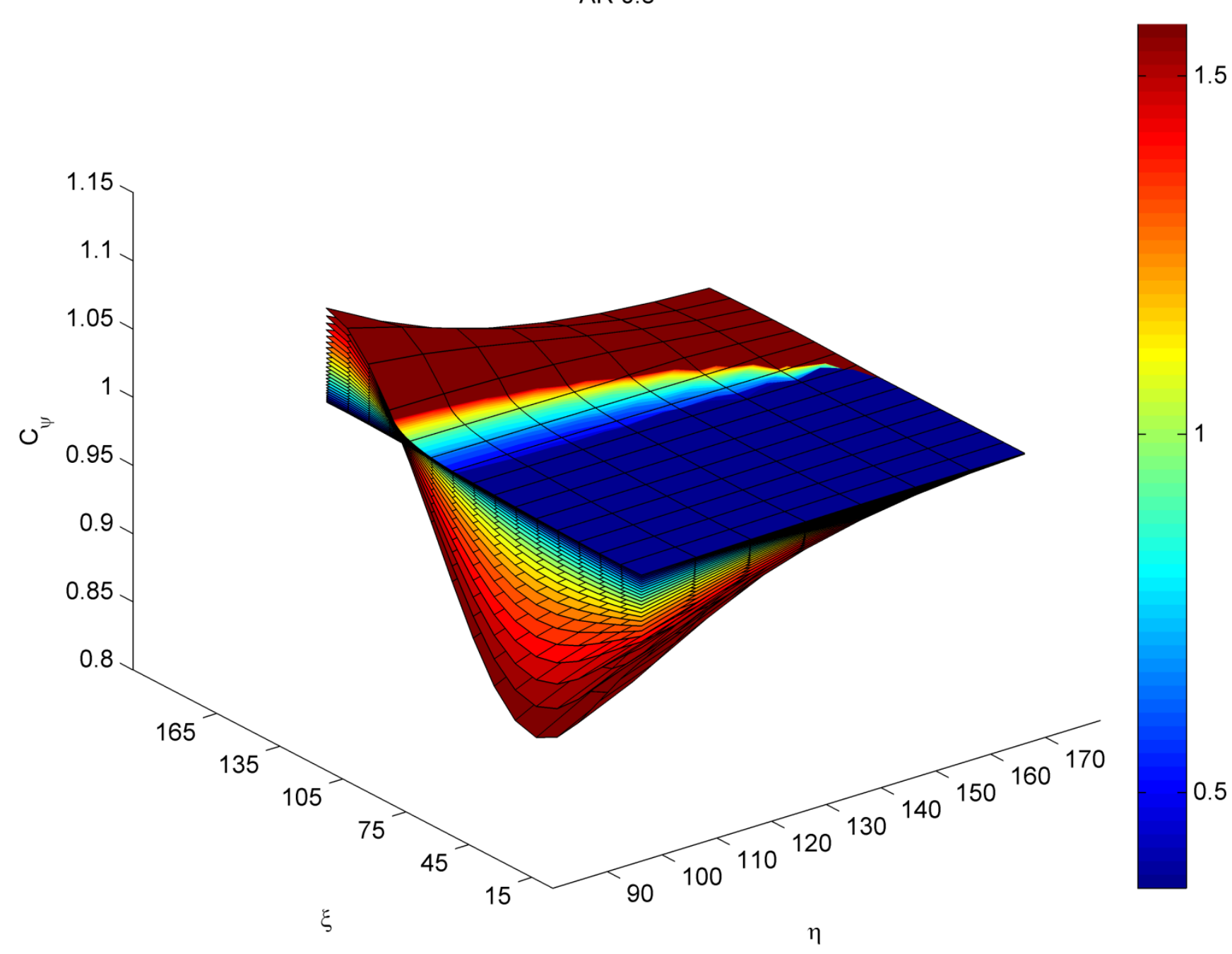

Figure $\mathrm{P} c_{\psi}$ for $A R 0.8$ 
AR 0.8

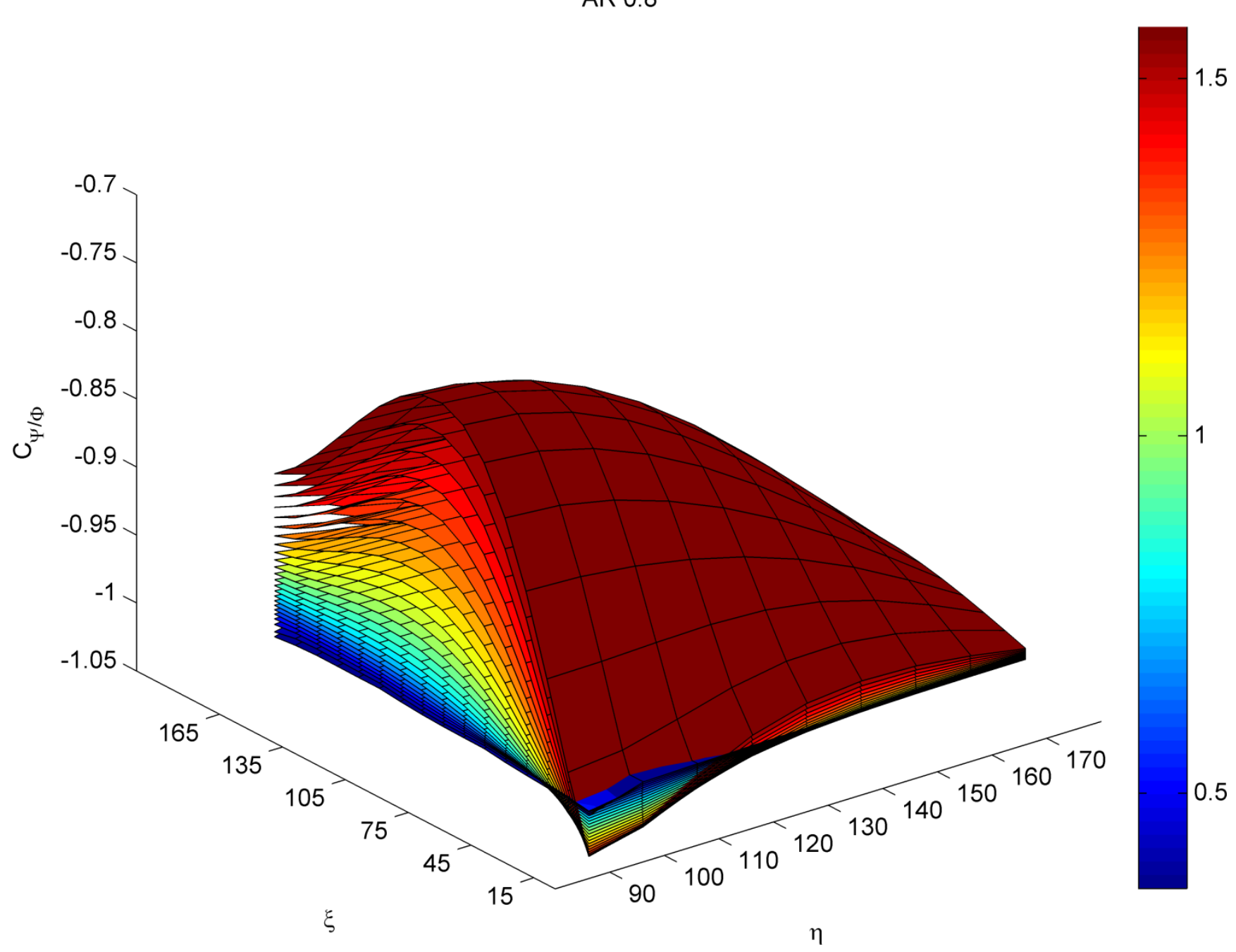

Figure $\mathrm{Q} c_{\Psi / \Phi}$ for $A R 0.8$ 


\section{AR 0.5}

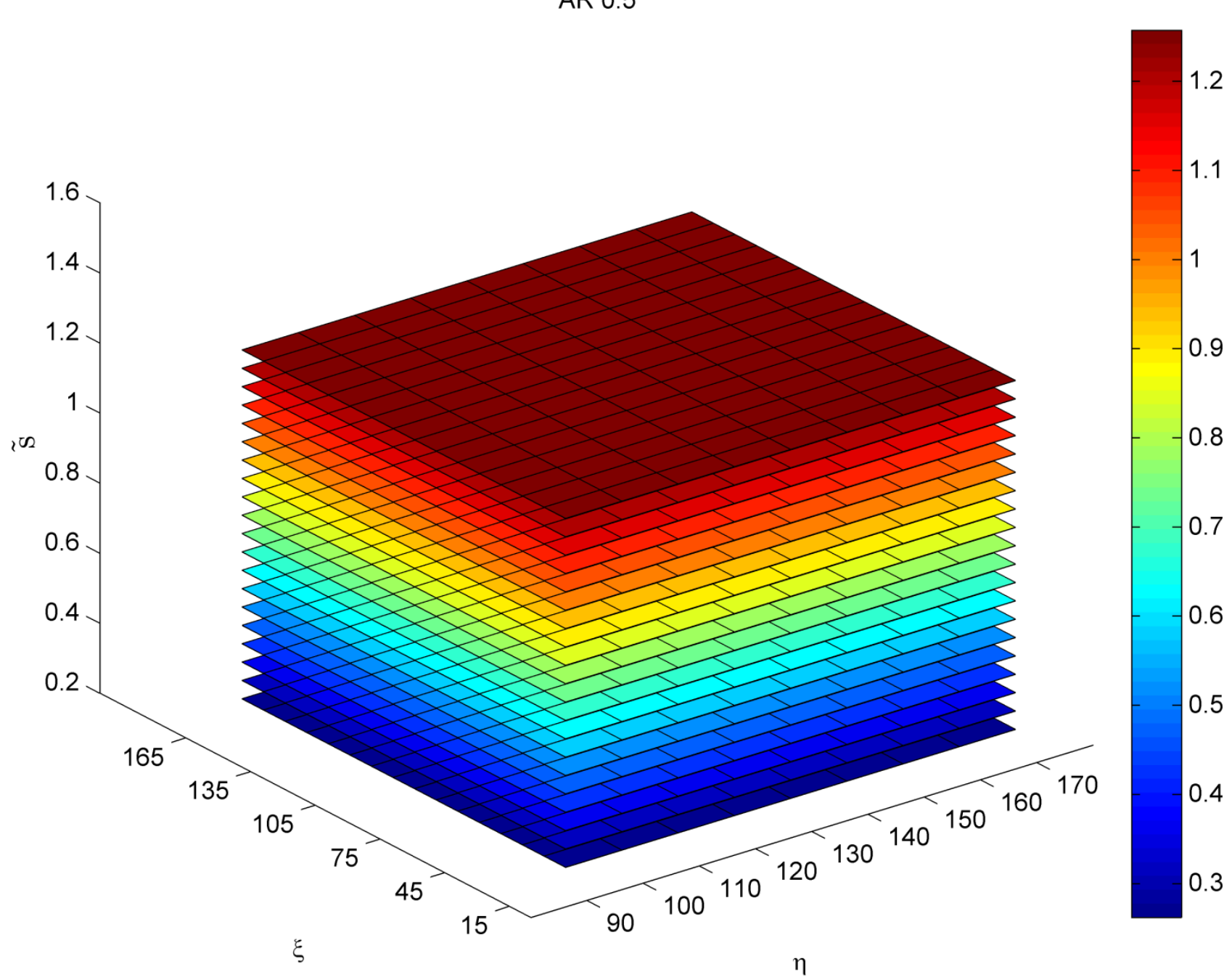

Figure R Nondimensional length for $A R 0.5$ 


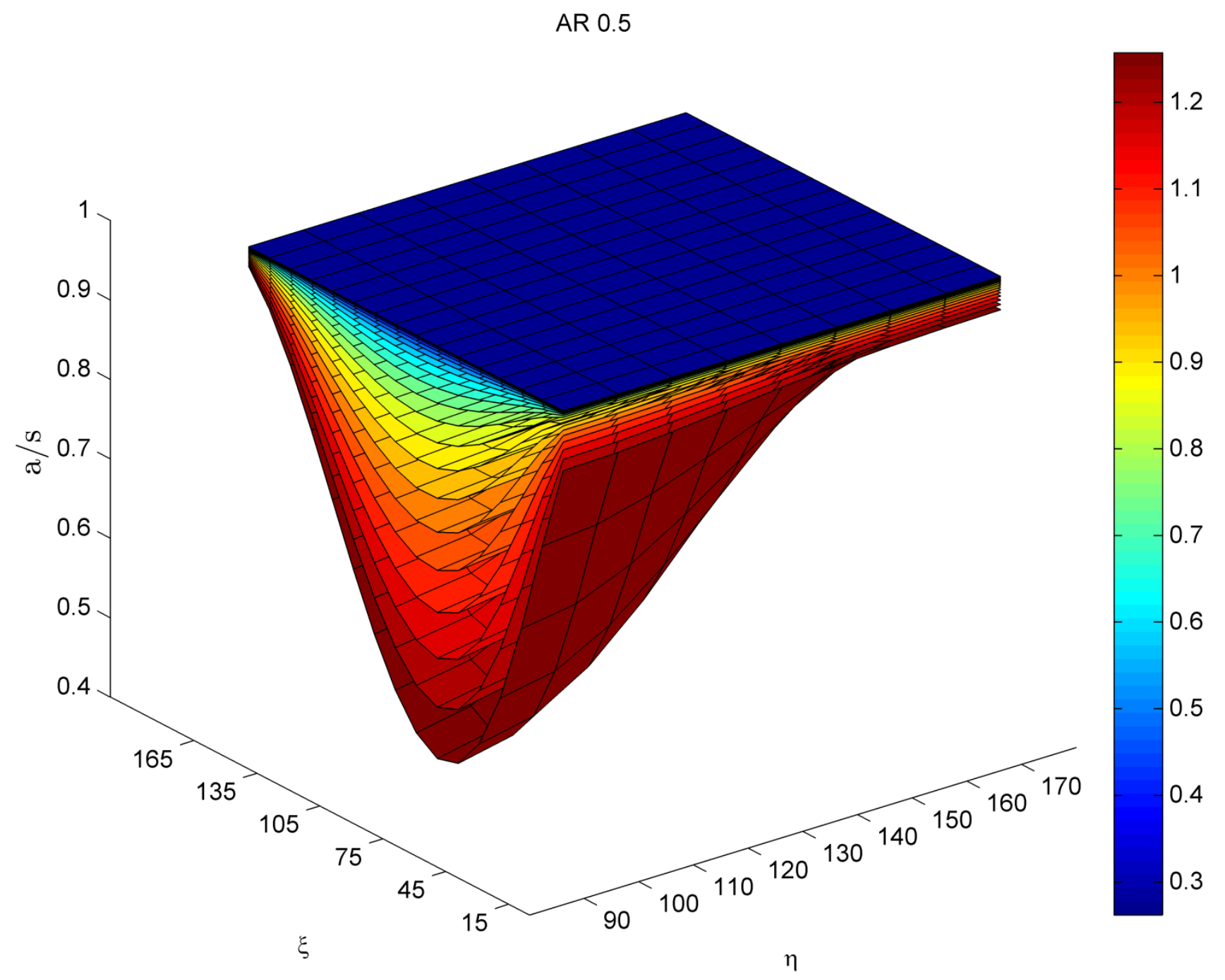

Figure $\mathrm{S} a / s$ for $A R 0.5$ 


\section{AR 0.5}

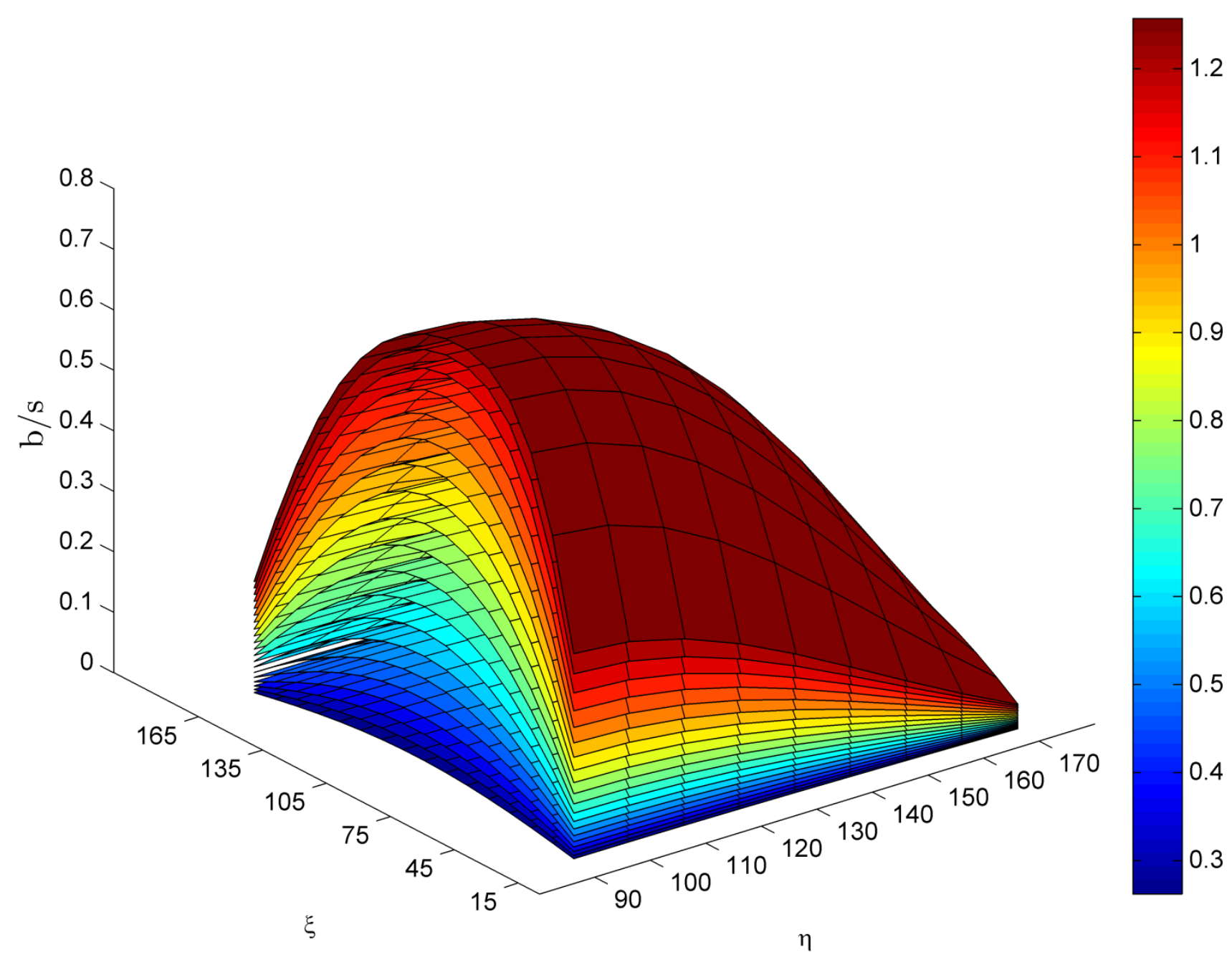

Figure $\mathrm{T} b / \mathrm{s}$ for $A R 0.5$ 


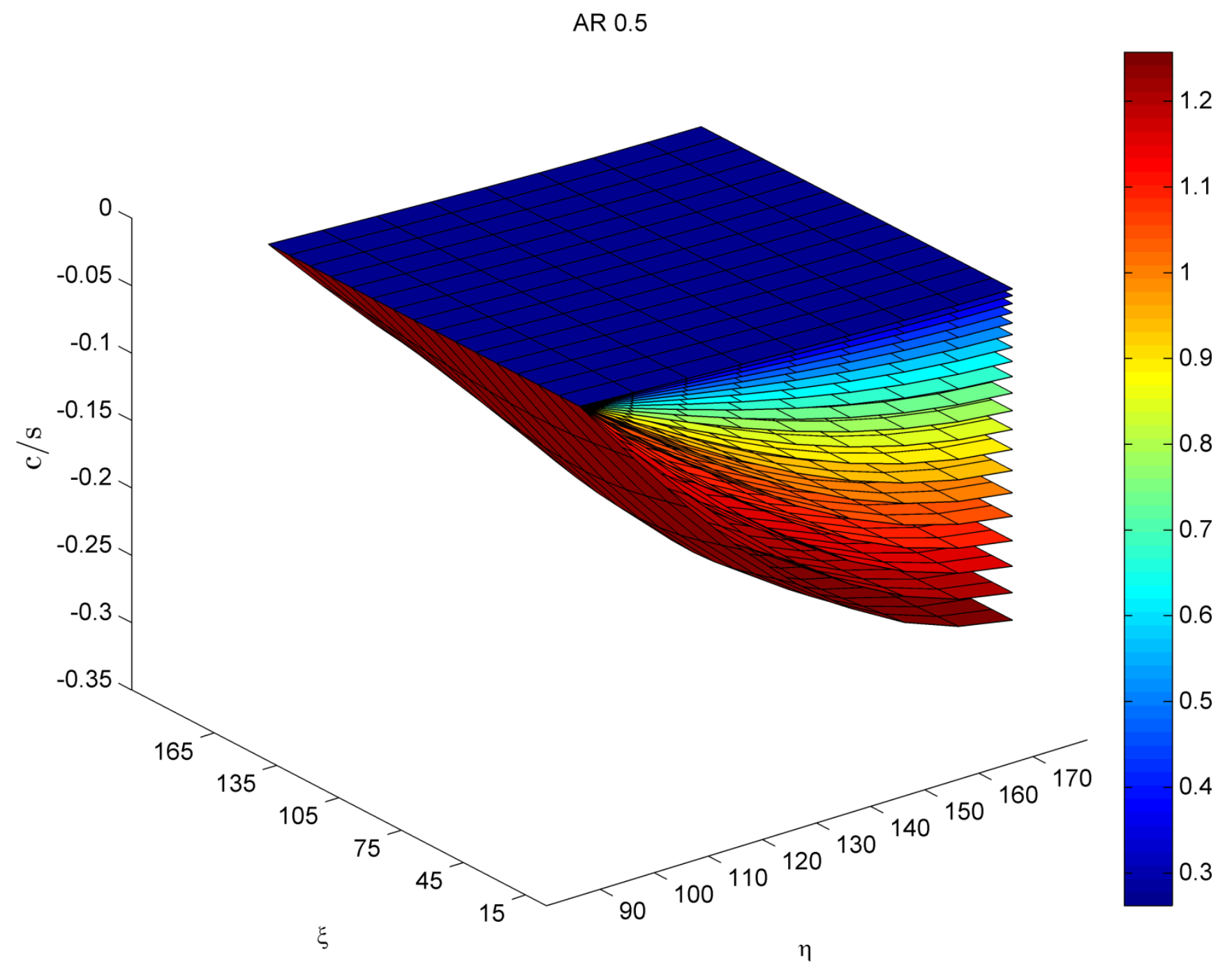

Figure $\mathrm{U} c / s$ for AR 0.5 
AR 0.5

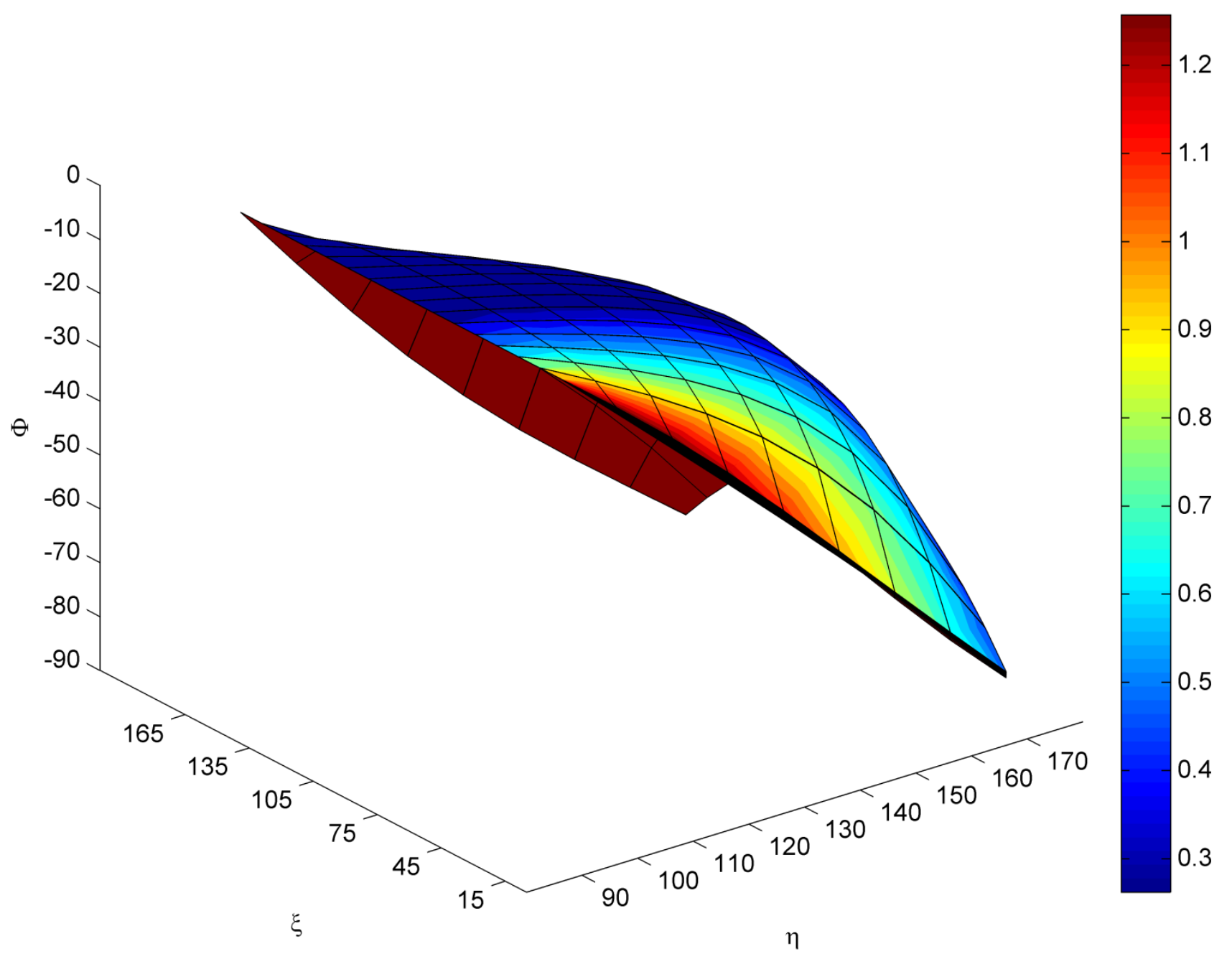

Figure V $\Phi$ for $A R 0.5$ 
AR 0.5

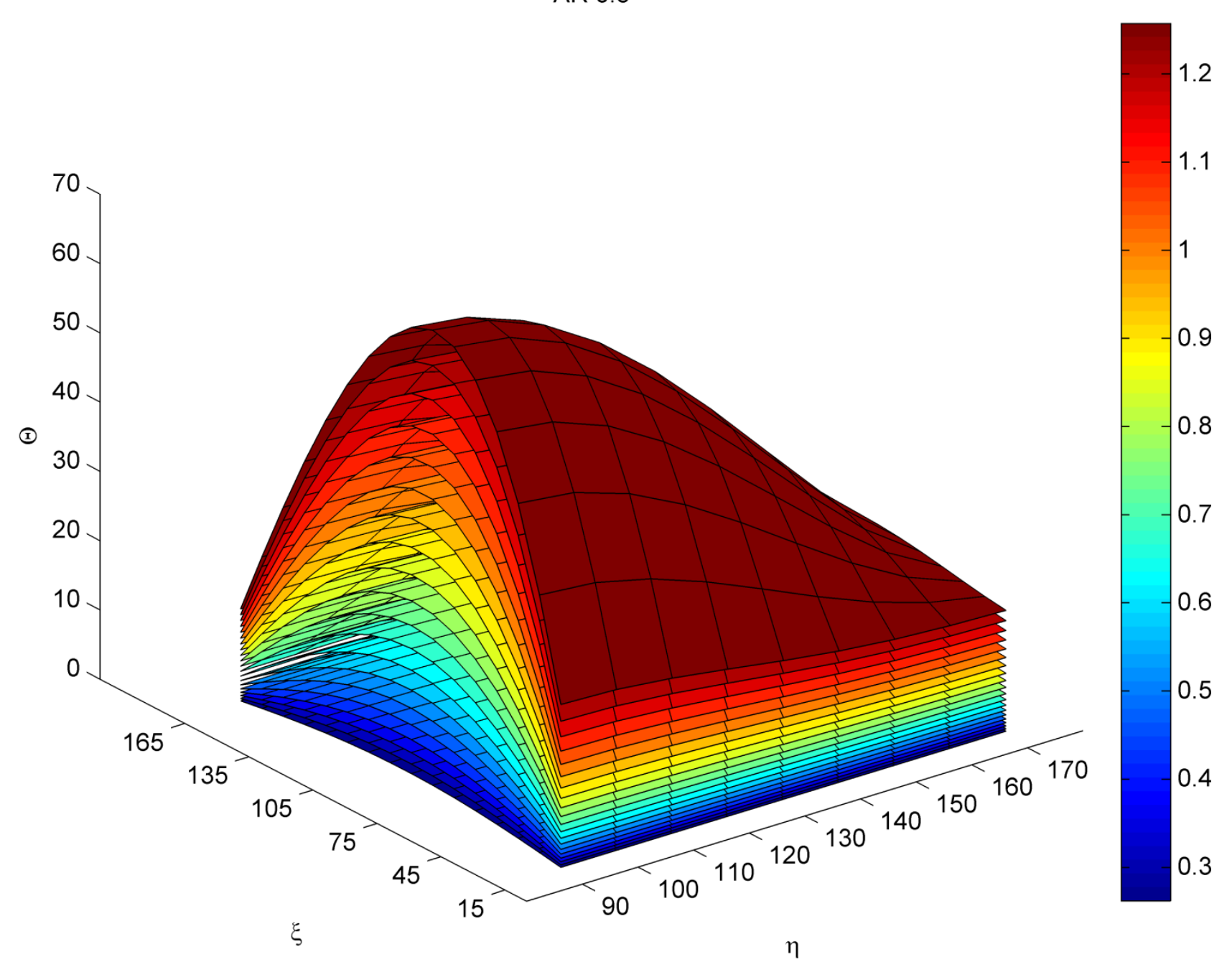

Figure $\mathrm{W} \Theta$ for $A R 0.5$ 


\section{AR 0.5}

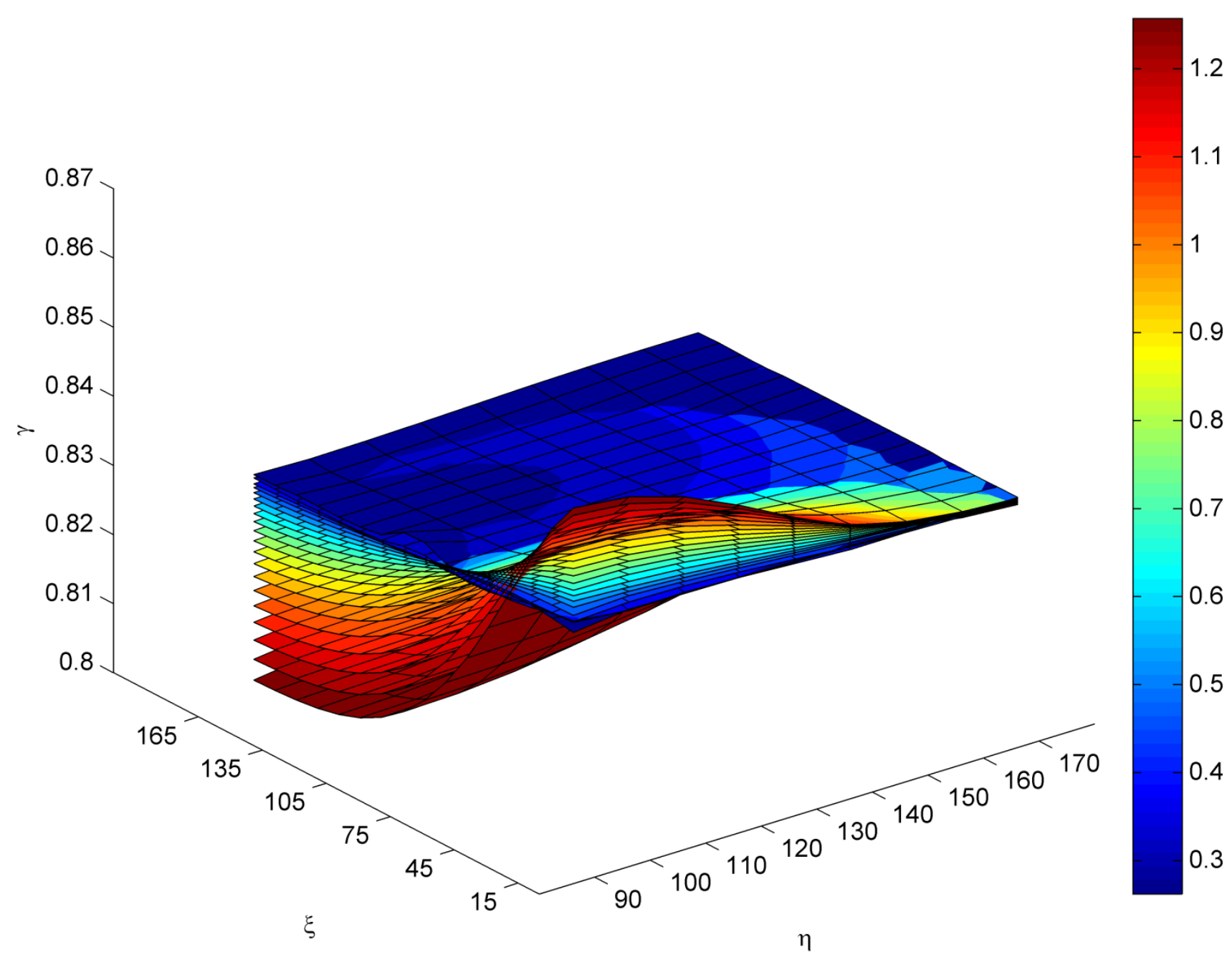

Figure $\mathrm{X} \gamma$ for $A R 0.5$ 
AR 0.5

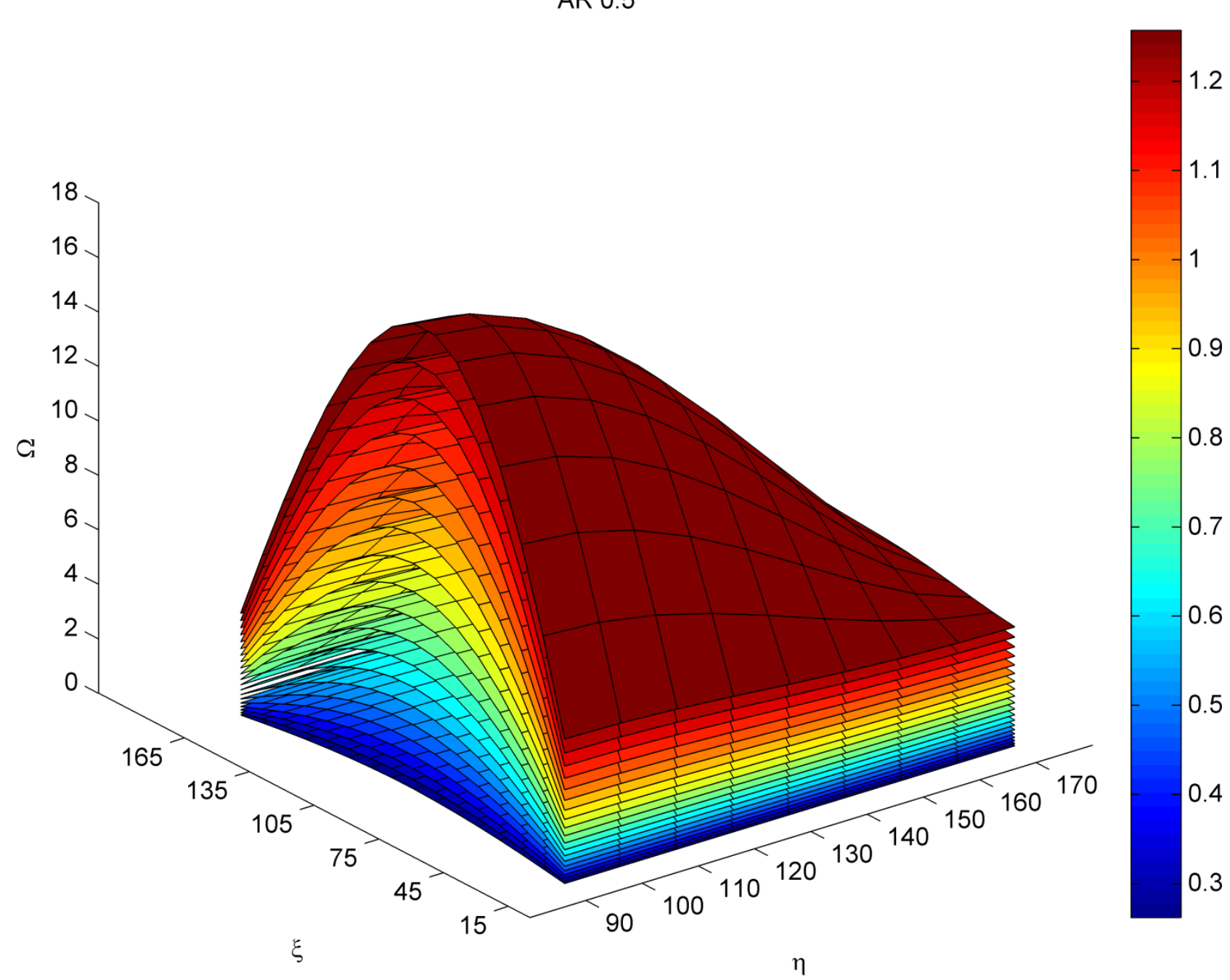

Figure $\mathrm{Y} \Omega$ for $A R 0.5$ 


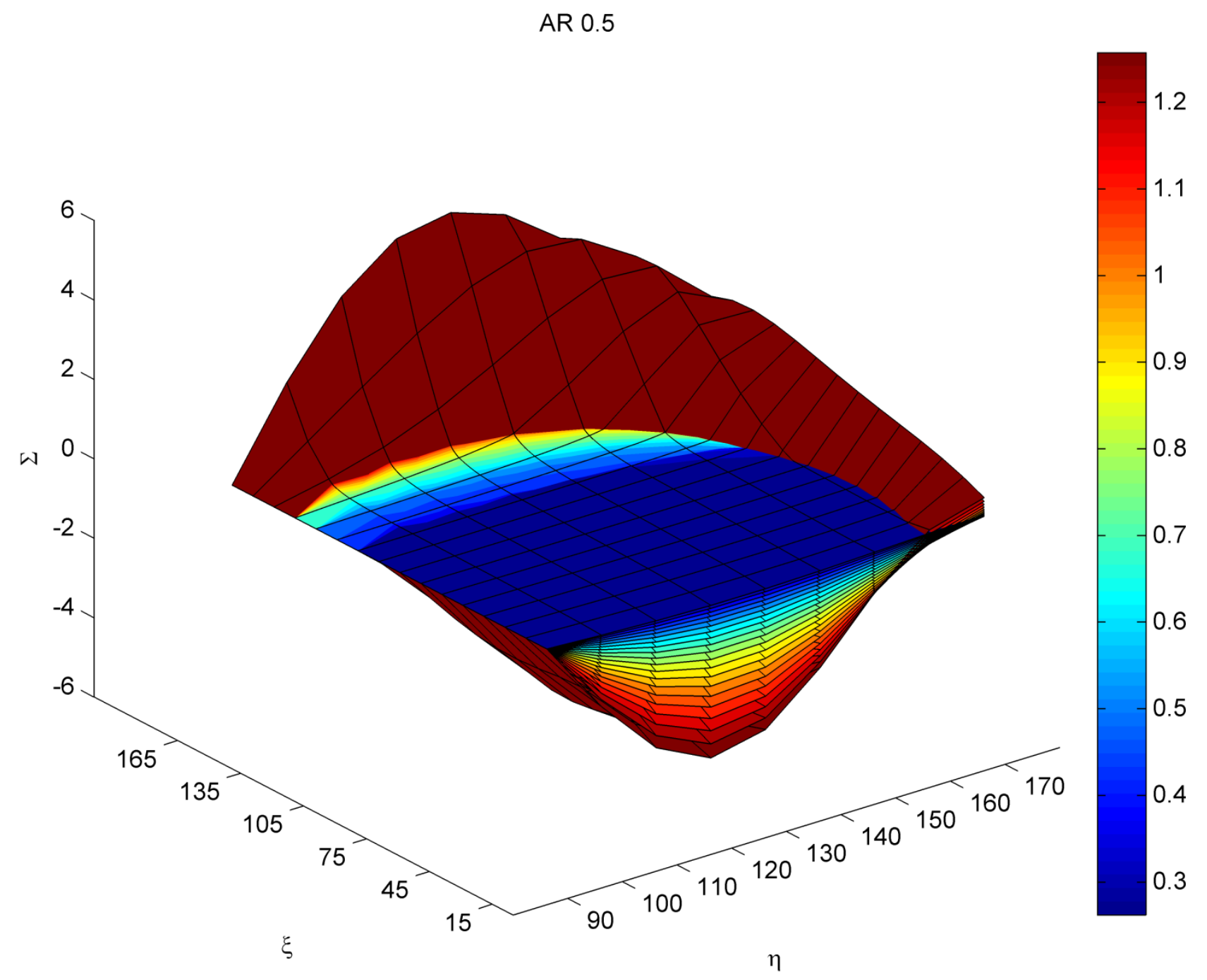

Figure $\mathrm{Z} \Sigma$ for $A R 0.5$ 


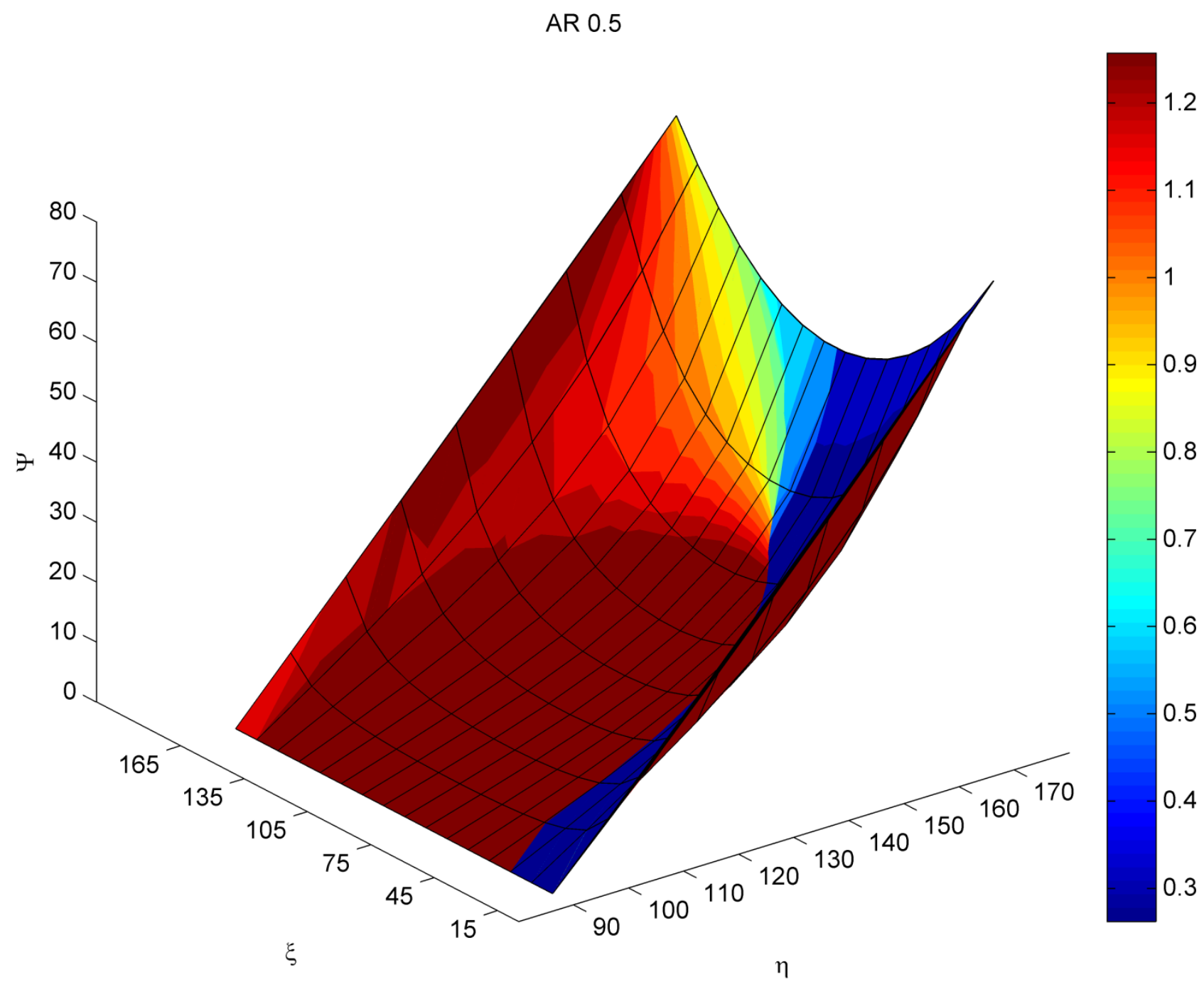

Figure AA $\Psi$ for $A R 0.5$ 


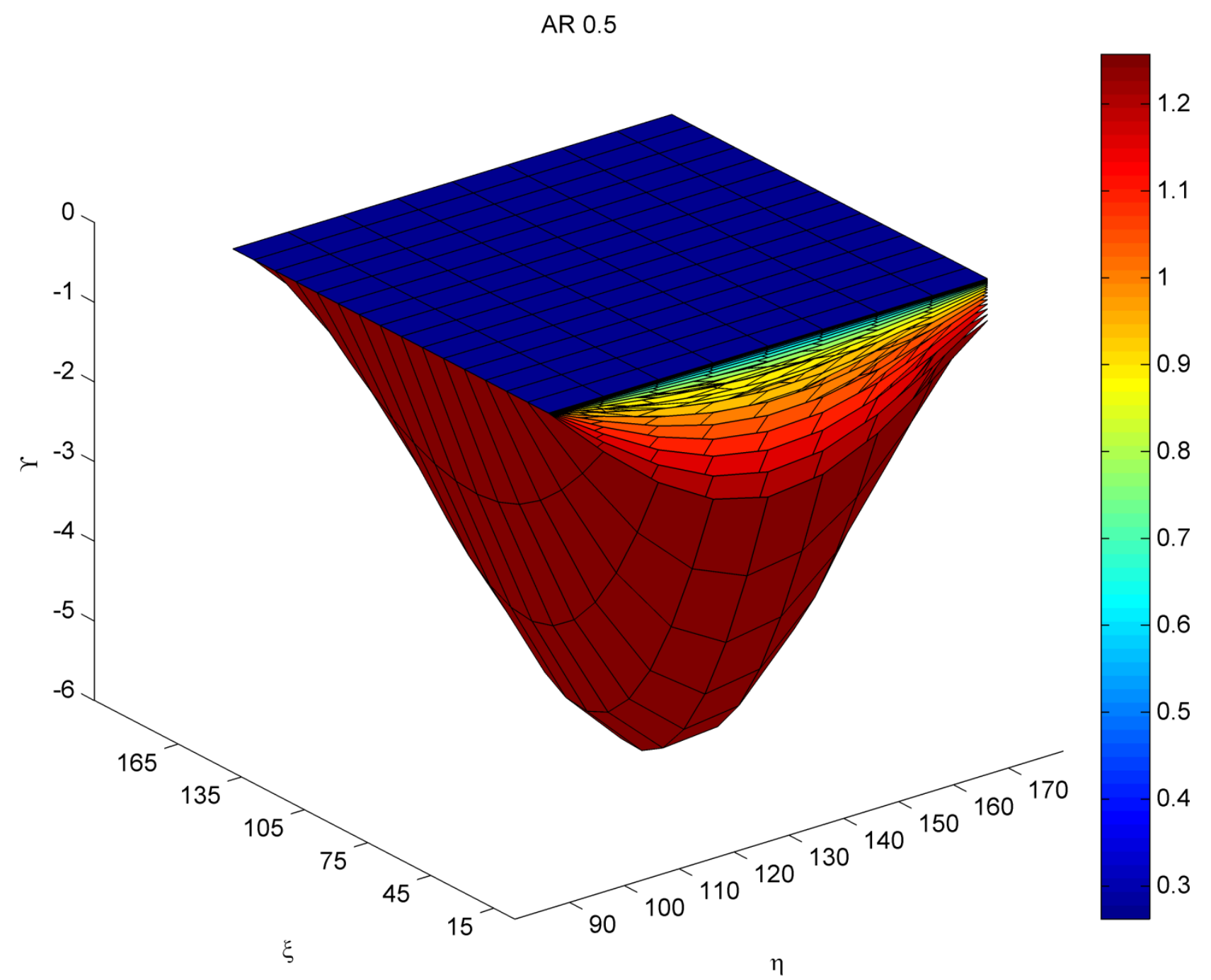

Figure $\mathrm{AB} \Upsilon$ for $A R 0.5$ 
AR 0.5

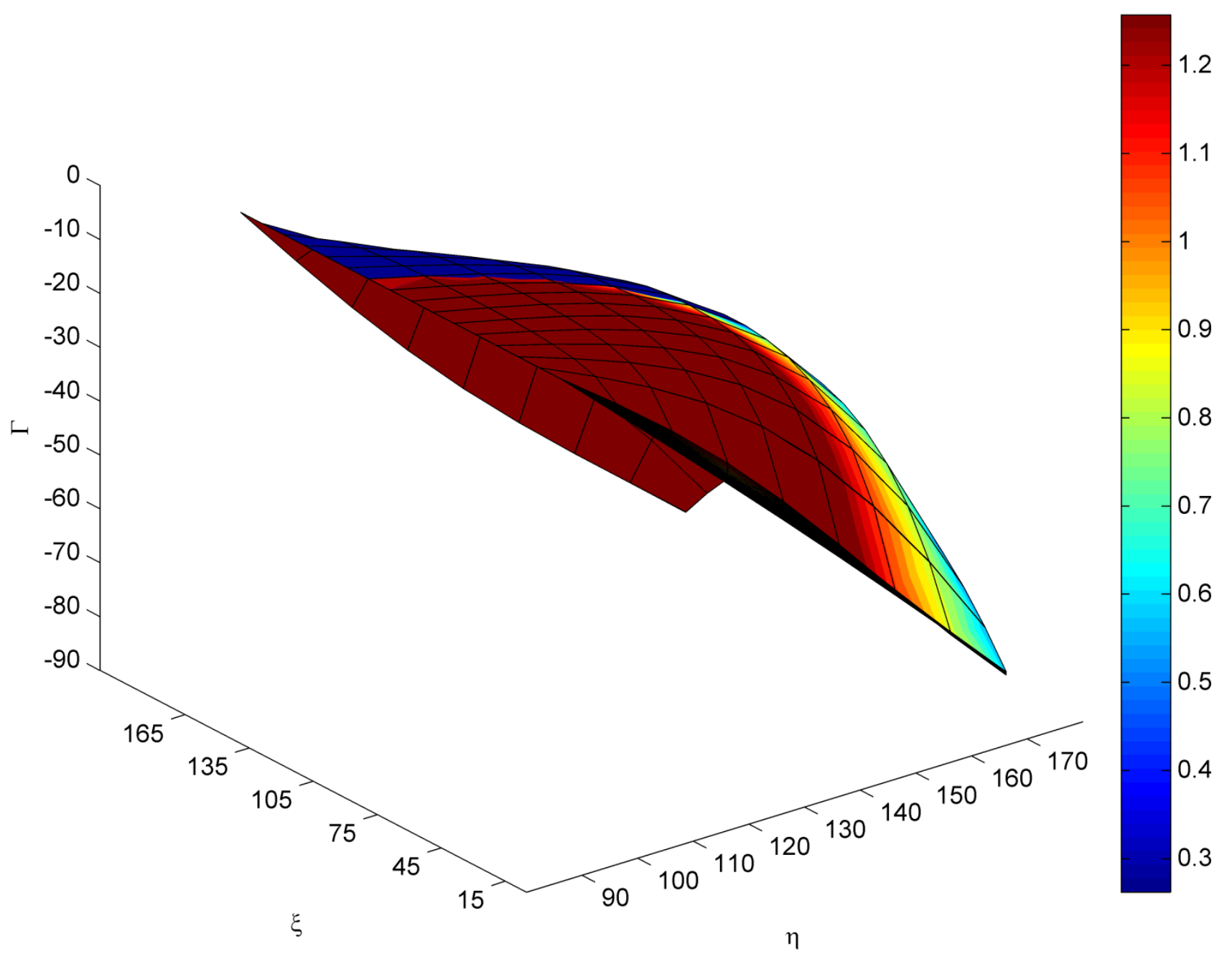

Figure AC $\Gamma$ for $A R 0.5$ 


\section{AR 0.5}

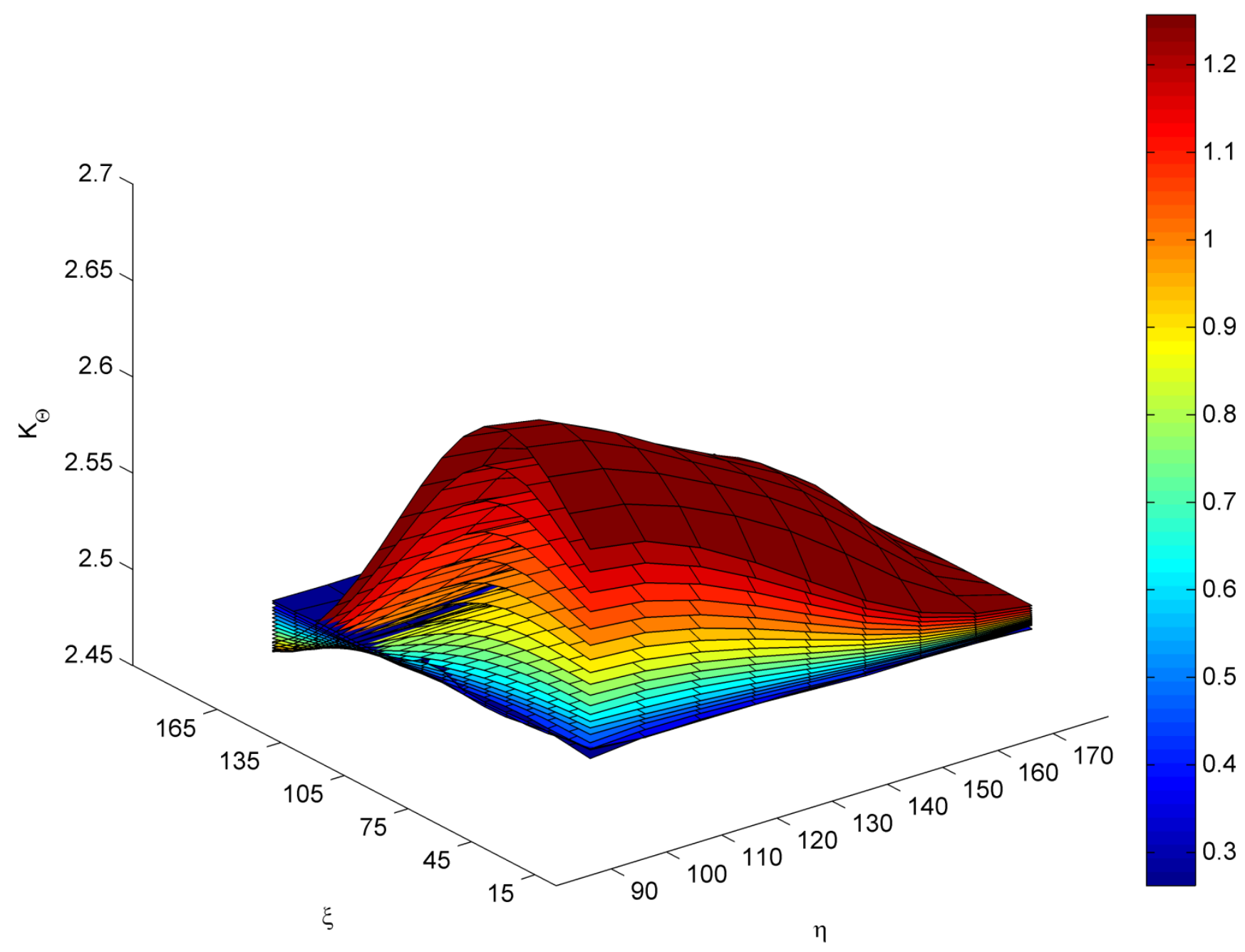

Figure AD $K_{\Theta}$ for $A R 0.5$ 
AR 0.5

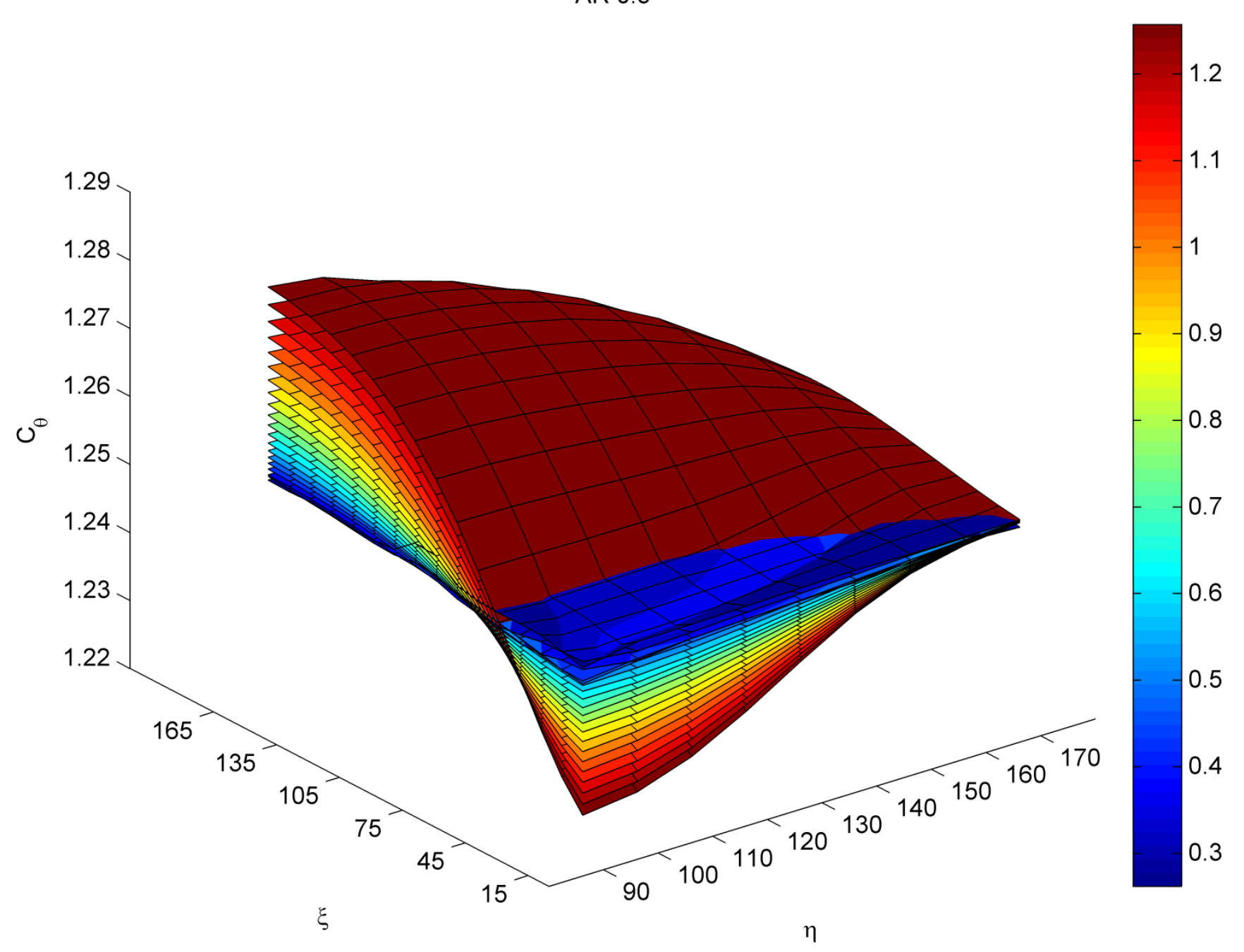

Figure $\mathrm{AE} c_{\theta}$ for $A R 0.5$ 
AR 0.5

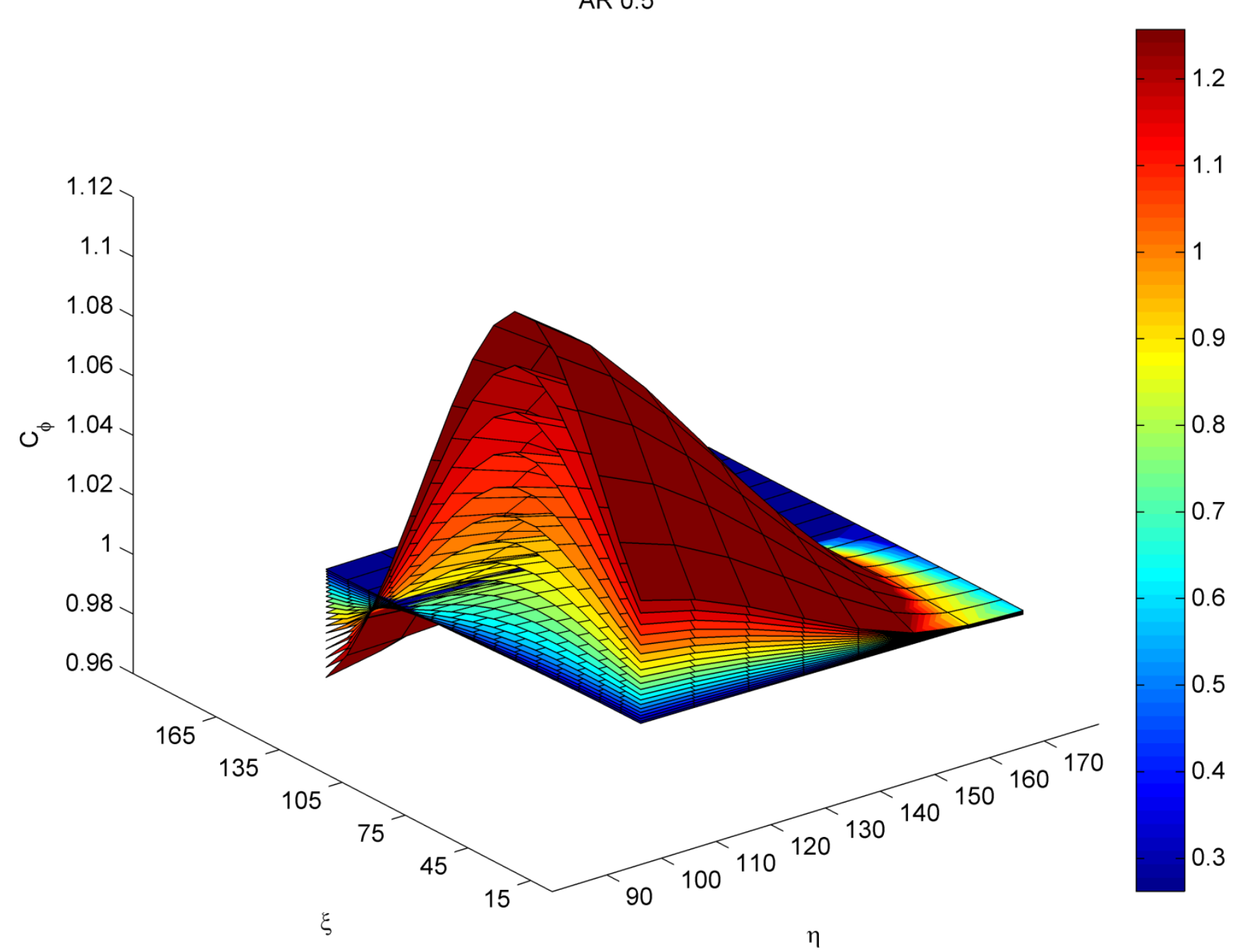

Figure $\mathrm{AF} c_{\phi}$ for $A R 0.5$ 


\section{AR 0.5}

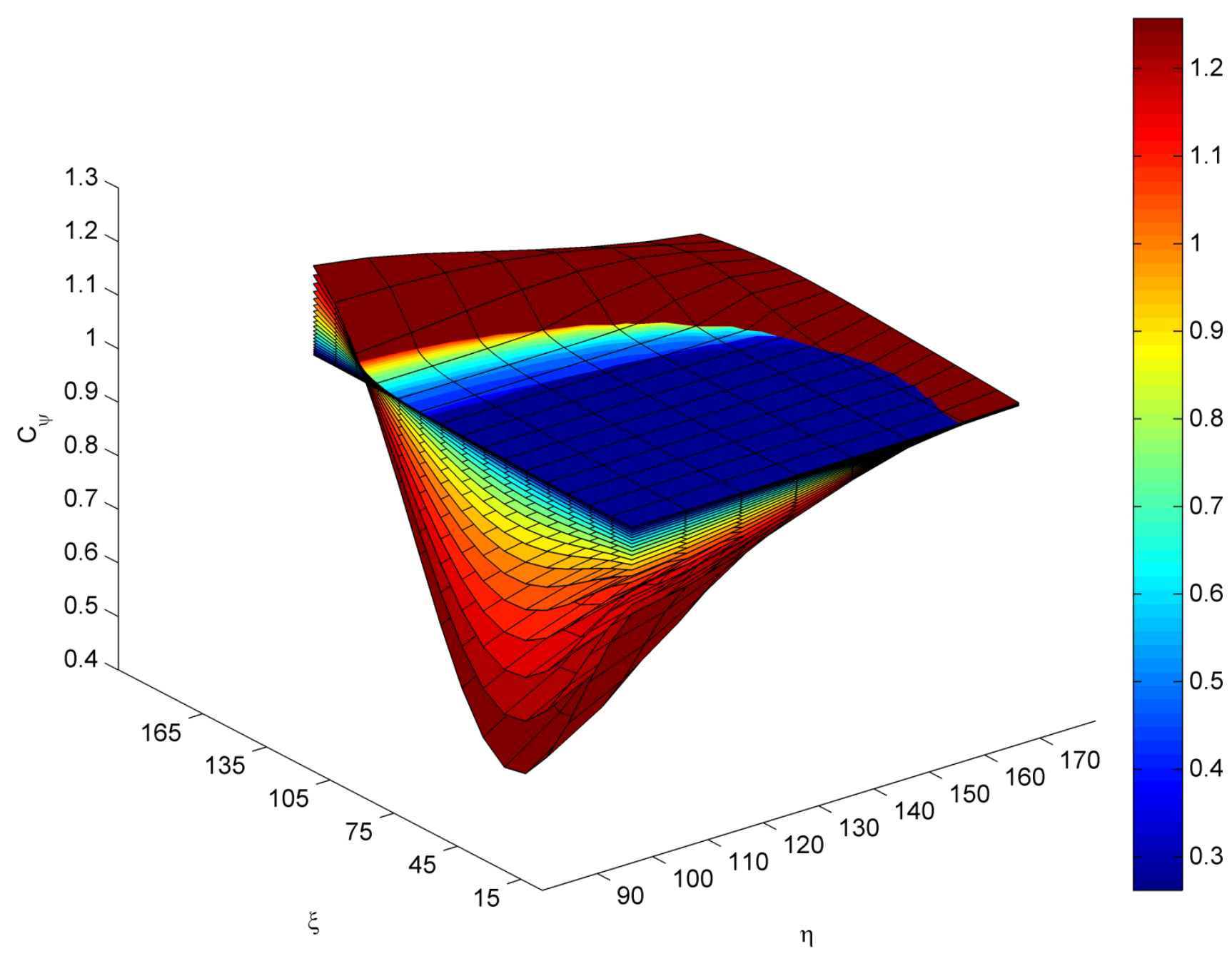

Figure AG $c_{\psi}$ for $A R 0.5$ 
AR 0.5

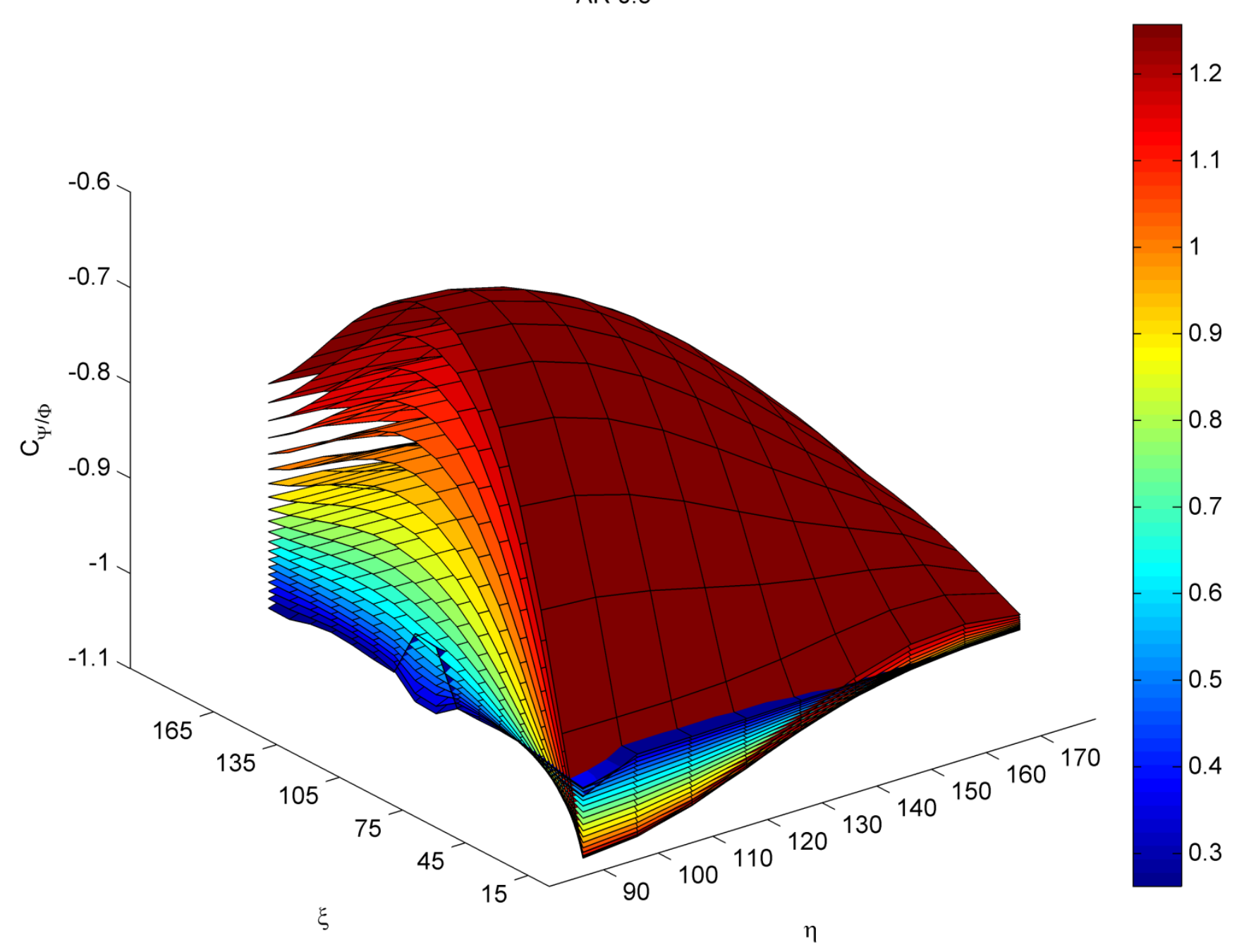

Figure $\mathrm{AH} c_{\Psi / \Phi}$ for $A R 0.5$ 


\section{Appendix D Code}

This is the Matlab code for numerical integration of nondimensional equations.

function three_D_eqns_non_dim(v);

$\%$ this function looks for the value of beam length in non_dimmensional-equations where curvature reaches maximum

$\%$ then uses the max beam legth to numericaly integrate the non_dimmensional-equations with greater detail.

clear all

clc

format longG

format compact

\%initial guess for total beam lenth

S_hat $=2 *$ pi;

for $\mathrm{AR}=[1,0.8,0.5,0.2] \%[1,0.8,0.5,0.2]$

max_s_layer=[];

$\%$ eta goes from 90 to 355 degrees

for eta $=$ degtorad([90:10:170])\%90:10:170

for $x i=$ degtorad([15:5:165])\%15:5:165

$\mathrm{v \_ i}=\operatorname{num} 2 \operatorname{str}(\operatorname{radtodeg}($ eta)$)$;

$v_{-} \mathrm{j}=\operatorname{num} 2 \operatorname{str}(\operatorname{radtodeg}(x i))$;

$\mathrm{v} \_\mathrm{k}=$ num2str(AR);

Cap_sigma $=[]$;

Cap_omega $=[]$;

Cap_psi =[];

\%number of points in the coarse integration along the beam length for finding initial k_hat_max and respective S_d_hat_max

num_iter_init = 9999;

$\%$ initialize matrices for integration result data

k1_hat $=[]$;

k2_hat $=[]$;

k3_hat $=[]$;

phi $=[]$;

theta $=[]$;

psi $=[]$;

h_X =[];

h_Y $=[]$;

$\mathrm{h} \_\mathrm{Z}=[]$;

h_S_hat_i $=[]$;

phi_i $=[]$;

theta_i =[];

psi_i $=[]$;

h_X_i $=[]$;

h_Y_i $=[]$;

h_Z_i $=[]$;

eta_xi_AR $=$ [radtodeg(eta),radtodeg(xi),AR]

$\%$ integrates each trajectory from a starting point on the $\mathrm{x}$-axis of the phase portrait 
[s_hat_i,q_hat_i] = ode45(@beam_3D_nondim,[0:-S_hat/num_iter_init:-S_hat],[0, 0, 0, 0, -1e-6, 0, 0, 0, 0]);

last_i=size(s_hat_i,1);

[pks0,locs0] = findpeaks(q_hat_i(:,3));

if eta $==$ degtorad(180)

end

[pks0,locs0] = findpeaks(q_hat_i(:,2));

if numel $(\operatorname{locs} 0)==0$

$\operatorname{locs} 0(1)=$ size(s_hat_i,1);

end

h_S_hat_max = s_hat_i $(\operatorname{locs} 0(1))$;

$\%$ h_S_hat_max $=-2.62$

num_iter $=50$;

h_S_hat $=[]$;

\%Integrates from free end to fixed end

[s_hat,q_hat] = ode45(@beam_3D_nondim,[0:h_S_hat_max/num_iter:h_S_hat_max],[0 , $0,0,0,-1 \mathrm{e}-6$, $0,0,0,0]$ );

last=size(s_hat,1);

$\%$ stores the results

\%curvatures at moving frame $\{\mathrm{A}\}$ which will be the fixed end of the beam

k1_hat $=[\mathrm{k} 1$ _hat, q_hat(:,1)];

k2_hat $=$ [k2_hat, q_hat(:,2)];

k3_hat $=$ [k3_hat, q_hat(:,3)];

\%euler angles from free $\{\mathrm{H}\}$ end end to 'moving' fixed end $\{\mathrm{A}\}$

phi $=$ [phi, q_hat(:,4)];

theta $=[$ theta, q_hat $(:, 5)]$;

psi = [psi, q_hat(:,6)];

\%Distance of 'moving' frame $\{\mathrm{A}\}$ seen from the frame $\{\mathrm{H}\}$ at the free end of the beam

h_X $=\left[\mathrm{h} \_X, \quad\right.$ q_hat(:,7)];

h_Y $=[$ h_Y, q_hat(:,8)];

h_Z =[h_Z, q_hat(:,9)];

\%Beam length in the negative $\mathrm{x}$ direction at frame $\{\mathrm{a}\}$

h_S_hat =[h_S_hat, s_hat];

a_pos = [];

a_F $=[]$;

d_F $=[]$;

\%iteration to find a, b and c viewd from Origin

for $\mathrm{i}=1$ :last

\%Rotation from $\{\mathrm{A}\}$ to $\{\mathrm{H}\}$ to express vectors from $\{\mathrm{H}\}$ to $\{\mathrm{A}\}$

$\mathrm{Cb}=\left[\begin{array}{lll}1 & 0 & 0\end{array}\right.$

$0 \cos ($ phi(i)) $\sin ($ phi(i))

0 -sin(phi(i)) cos(phi(i))];

$\mathrm{Bb}=[\cos ($ theta(i)) $\sin ($ theta(i)) 0

-sin(theta(i)) $\cos ($ theta(i)) 0

$\left.\begin{array}{lll}0 & 0 & 1\end{array}\right]$

$\mathrm{Ab}=\left[\begin{array}{lll}1 & 0 & 0\end{array}\right.$

$0 \cos (\mathrm{psi}(\mathrm{i})) \sin (\mathrm{psi}(\mathrm{i}))$

0 -sin(psi(i)) cos(psi(i))];

a_pos $=$ [a_pos;[-Cb*Bb*Ab*[h_X(i,1);h_Y(i,1);h_Z(i,1)]]'];

end

$\%$ vector position of free end of the beam seen from frame $\{\mathrm{A}\}$ 


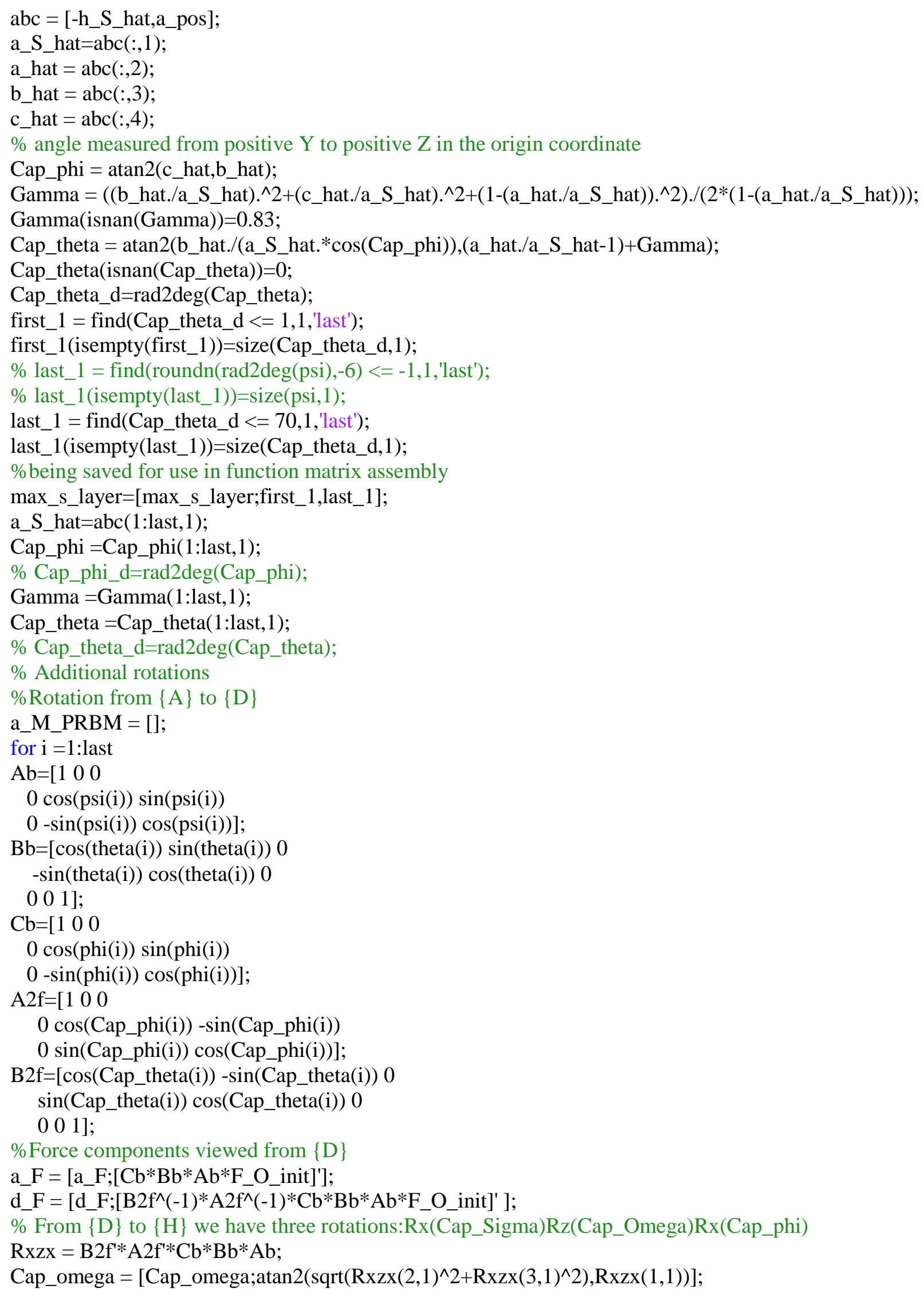




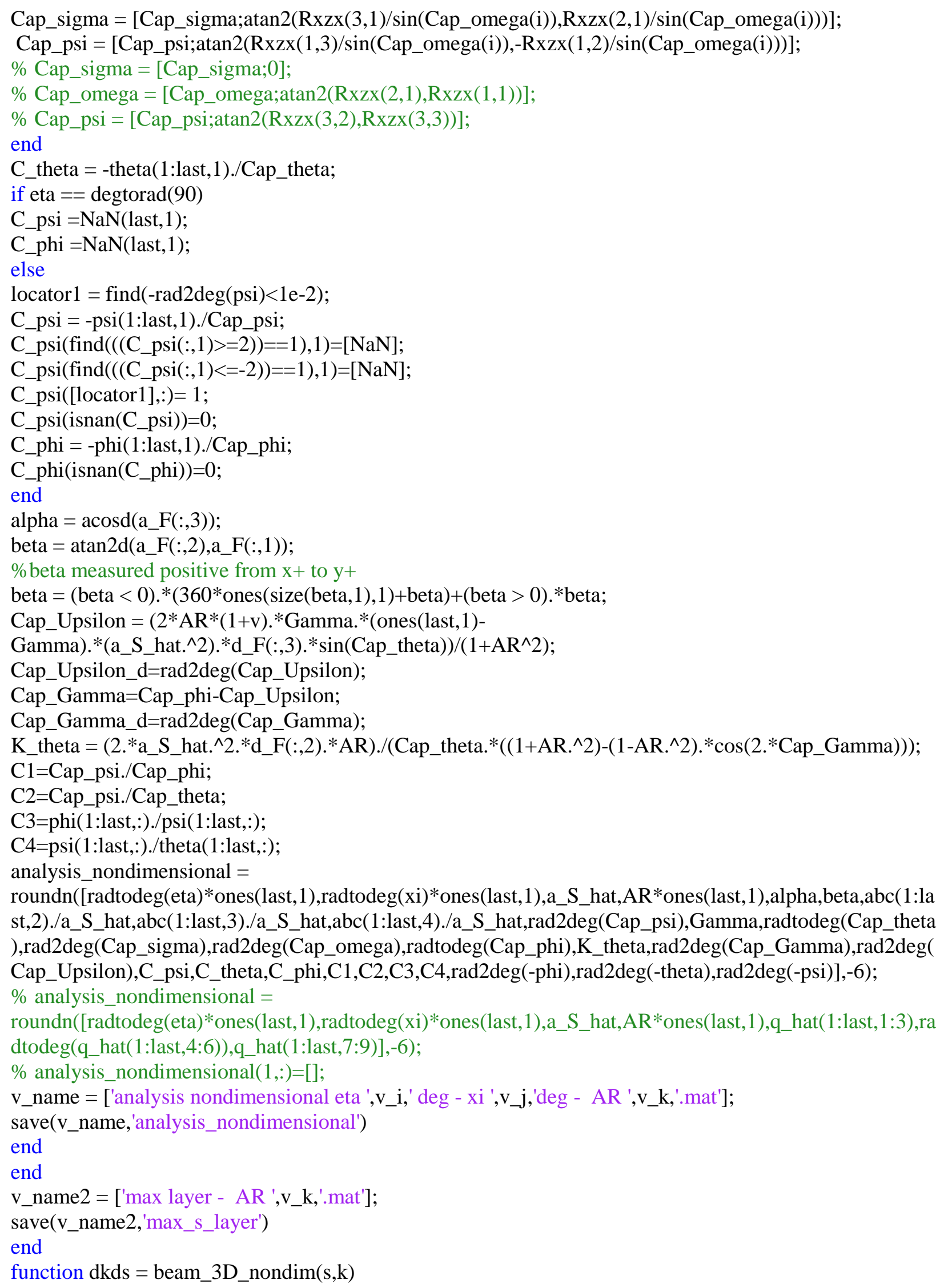


$\%$ Applied force at the beam end viewed from the origin before the beam starts the defection

F_O_init $=[\sin ($ eta $) * \cos (x i) ; \sin ($ eta $) * \sin (x i) ; \cos ($ eta $)]$;

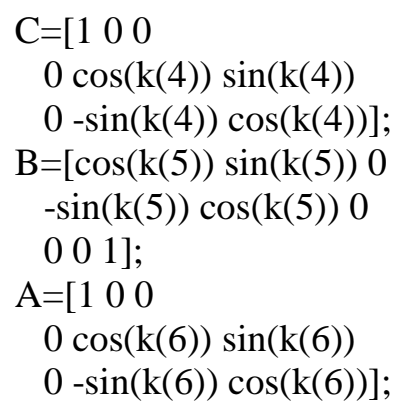

$\%$ Follower Force at beam end viewed from the origin. Shows how it changes direction as it deflects the beam.

F_O = C*B*A*F_O_init;

Fy_prime $=$ F_O(2);

Fz_prime = F_O(3);

$\mathrm{v}=0.3$;

dkds = zeros(size $(\mathrm{k}))$;

\%curvature with respect to origin because of how load is applied.

d_k $1=((2 *(1+\mathrm{v}) *(1-\mathrm{AR} \wedge 2)) /(1+\mathrm{AR} \wedge 2)) * \mathrm{k}(2) * \mathrm{k}(3)$;

d_k2 $=\left(\mathrm{AR}^{\wedge} 2-\left(\left(1+\mathrm{AR}^{\wedge} 2\right) /(2 *(1+\mathrm{v}))\right)\right) * \mathrm{k}(1) * \mathrm{k}(3)+\mathrm{AR} * \mathrm{Fz} \_$prime;

d_k3 $=((A R \wedge 2-2 * v-1) /(2 *(1+v) * A R \wedge 2)) * k(1) * k(2)-F y \_p r i m e /(A R)$;

d_phi $=\mathrm{k}(1)+(\mathrm{k}(2) * \cos (\mathrm{k}(4)) * \cos (\mathrm{k}(5))-\mathrm{k}(3) * \sin (\mathrm{k}(4)) * \cos (\mathrm{k}(5))) / \sin (\mathrm{k}(5))$;

d_theta $=\mathrm{k}(2) * \sin (\mathrm{k}(4))+\mathrm{k}(3) * \cos (\mathrm{k}(4))$;

d_psi $=(-\mathrm{k}(2) * \cos (\mathrm{k}(4))+\mathrm{k}(3) * \sin (\mathrm{k}(4))) / \sin (\mathrm{k}(5))$;

d_X $=\cos (k(5))$;

d_Y $=\cos (k(6)) * \sin (k(5))$;

d_Z $=\sin (k(6)) * \sin (k(5))$;

dkds $=[$ d_k1 d_k2 d_k3 d_phi d_theta d_psi d_X d_Y d_Z]';

end

end

This is the Matlab code for matrix assembly.

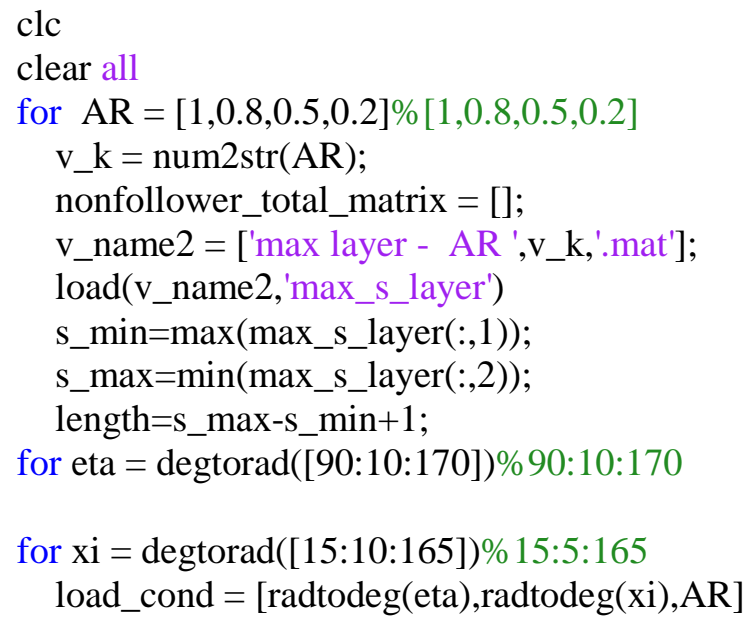




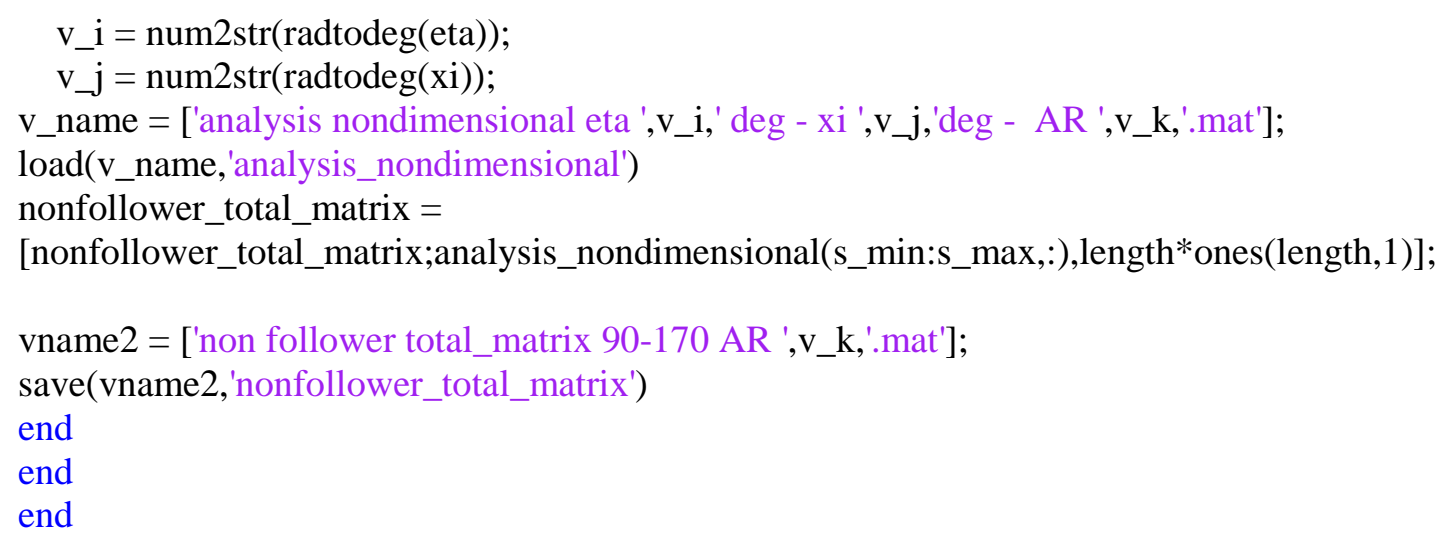

This is the Matlab code for surface plotting.

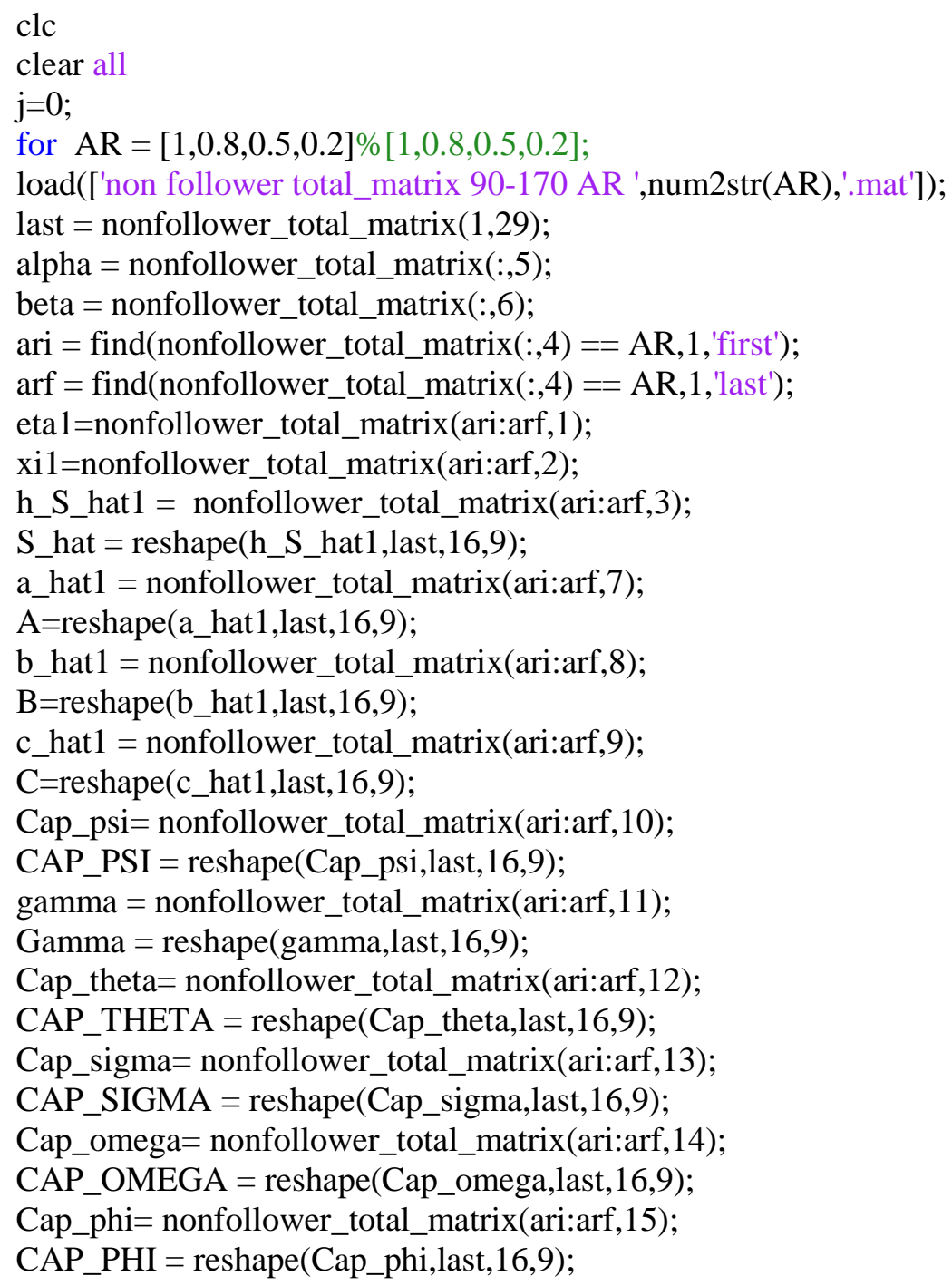


K_theta= nonfollower_total_matrix(ari:arf,16);

K_THETA = reshape(K_theta,last,16,9);

Cap_Gamma= nonfollower_total_matrix(ari:arf,17);

CAP_GAMMA = reshape(Cap_Gamma,last,16,9);

Cap_Upsilon= nonfollower_total_matrix(ari:arf,18);

CAP_UPSILON = reshape(Cap_Upsilon,last,16,9);

C_psi= nonfollower_total_matrix(ari:arf,19);

C_PSI = reshape(C_psi,last,16,9);

C_theta= nonfollower_total_matrix(ari:arf,20);

C_THETA = reshape(C_theta,last,16,9);

C_phi= nonfollower_total_matrix(ari:arf,21);

C_PHI = reshape(C_phi,last,16,9);

C_1= nonfollower_total_matrix(ari:arf,22);

C_1 = reshape(c_1,last,16,9);

iter $=8$;

for $\mathrm{i}=$ [last:-floor((last-1)/iter):1]

figure $(1+17 * \mathrm{j})$;

title(['AR ',num2str(AR)])

hold all

surf(squeeze(A(i,:::)),squeeze(S_hat(i,:::)))

xlabel('ไeta' )

set(gca,'XTick',1:1:9)

set(gca,'XTickLabel',\{'90','100','110','120','130','140','150','160','170'\})

ylabel('Ixi')

set(gca,'YTick',1:3:16)

set(gca,'YTickLabel',\{'15','45','75','105','135','165'\})

zlabel('a/s','interpreter','latex')

colorbar

view(3)

if $\mathrm{i}<=(($ last-1)/iter)

set(gcf,'PaperType','usletter')

print('-dtiff','-r600',['1a_hat_AR',num2str(10*AR)]);

end

hold off

figure $(2+17 * \mathrm{j})$

title(['AR ',num2str(AR)])

hold all

surf(squeeze(B(i,:,:)),squeeze(S_hat(i,:;:)))

xlabel('leta' )

set(gca,'XTick',1:1:9)

set(gca,'XTickLabel', \{'90','100','110','120','130','140','150','160','170'\})

ylabel('|xi')

set(gca,'YTick',1:3:16)

set(gca,'YTickLabel',\{'15','45','75','105','135','165'\})

zlabel('b/s','interpreter','latex')

colorbar

view(3)

if $\mathrm{i}<=(($ last-1)/iter $)$

set(gcf,'PaperType','usletter')

print('-dtiff','-r600',['2b_hat_AR',num2str(10*AR)]);

end 


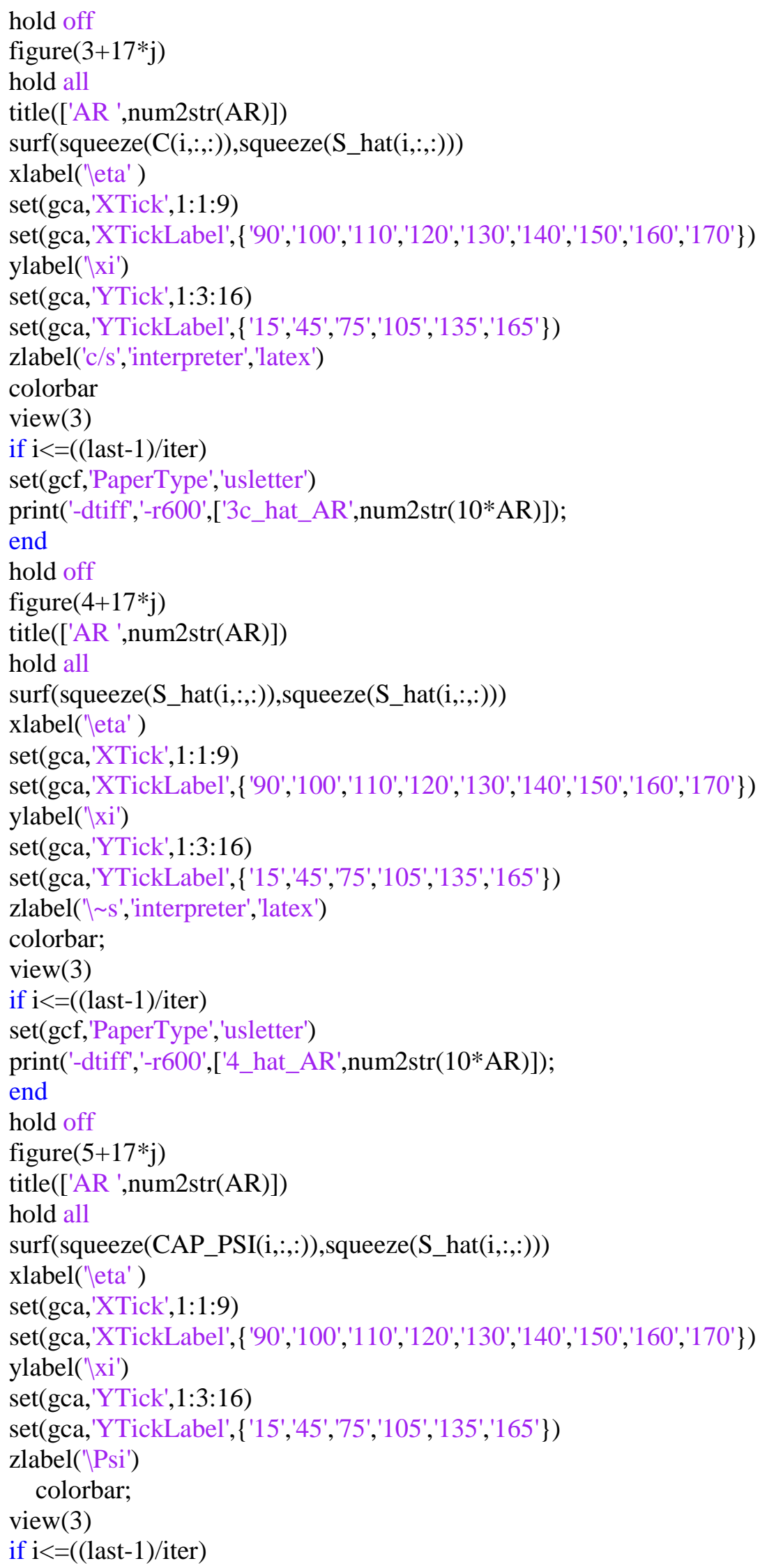




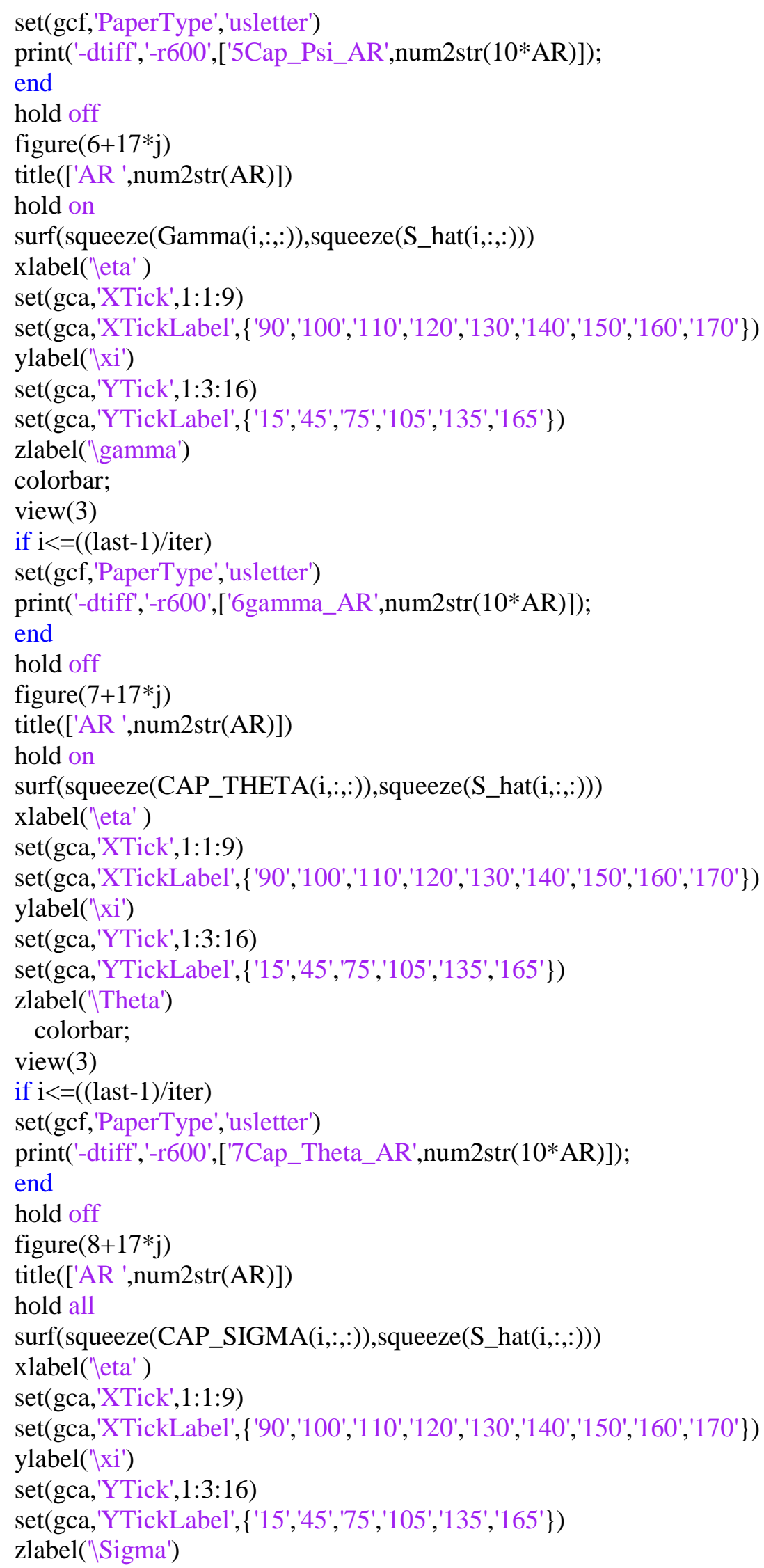




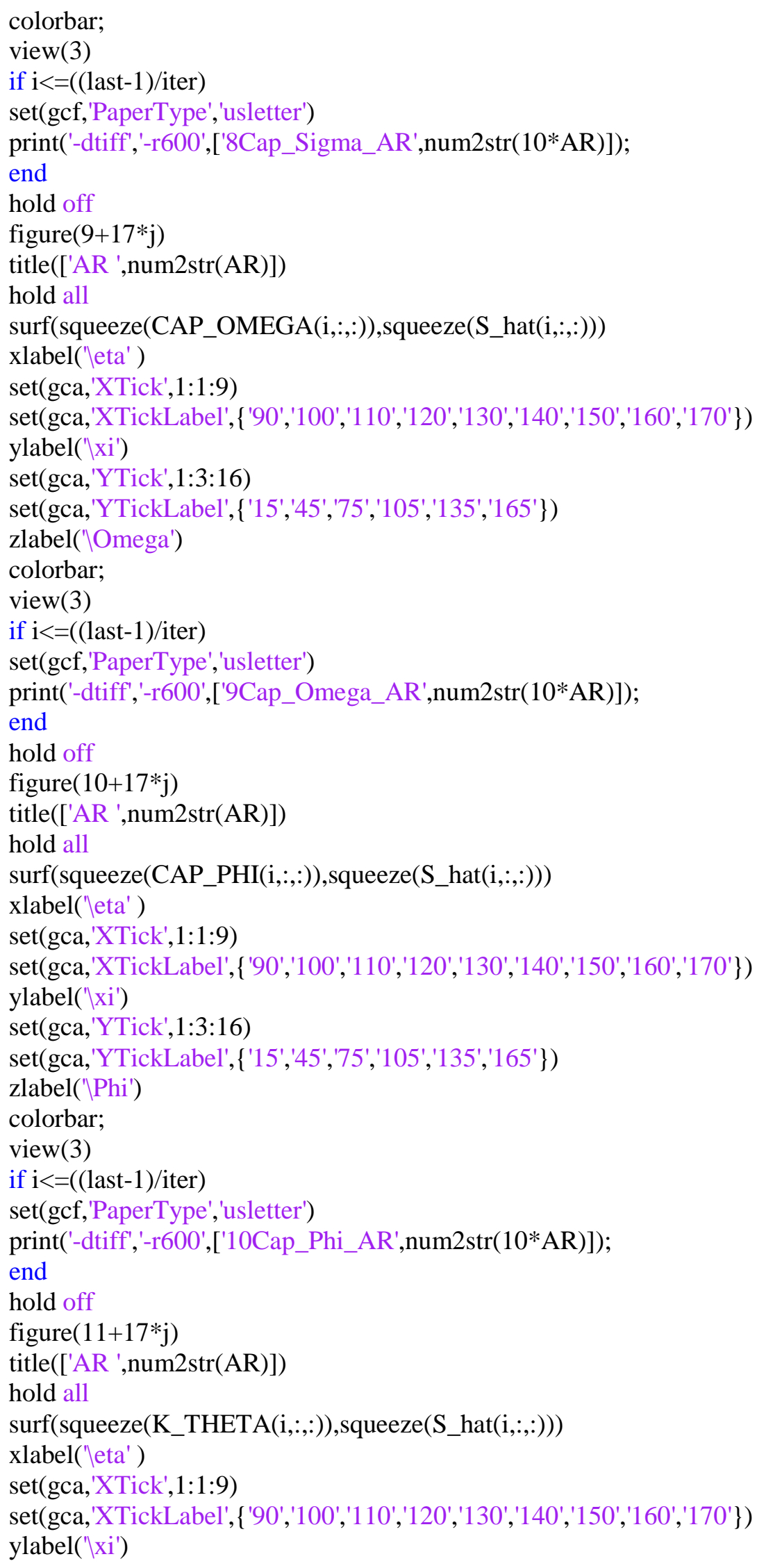


set(gca,'YTick',1:3:16)

set(gca,'YTickLabel', \{'15','45','75','105','135','165'\})

zlabel('K_ıTheta')

colorbar;

view(3)

if $\mathrm{i}<=(($ last-1)/iter $)$

set(gcf,'PaperType','usletter')

print('-dtiff','-r600',['11K_theta_AR',num2str(10*AR)]);

end

hold off

figure $(12+17 * \mathrm{j})$

title(['AR ',num2str(AR)])

hold all

surf(squeeze(CAP_GAMMA(i,::;)),squeeze(S_hat(i,:,:)))

xlabel('ไeta' )

set(gca,'XTick',1:1:9)

set(gca,'XTickLabel',\{'90','100','110','120','130','140','150','160','170'\})

ylabel('Ixi')

set(gca,'YTick',1:3:16)

set(gca,'YTickLabel',\{'15','45','75','105','135','165'\})

zlabel('IGamma')

colorbar;

view(3)

if $\mathrm{i}<=(($ last-1)/iter $)$

set(gcf,'PaperType','usletter')

print('-dtiff','-r600',['12Cap_Gamma_AR',num2str(10*AR)]);

end

hold off

figure $(13+17 * \mathrm{j})$

title(['AR ',num2str(AR)])

hold all

surf(squeeze(CAP_UPSILON(i,:,:)),squeeze(S_hat(i,:,:)))

xlabel('leta' )

set(gca,'XTick',1:1:9)

set(gca,'XTickLabel',\{'90','100','110','120','130','140','150','160','170'\})

ylabel('|xi')

set(gca,'YTick',1:3:16)

set(gca,'YTickLabel',\{'15',45','75','105','135','165'\})

zlabel('UUpsilon ')

colorbar;

view(3)

if $\mathrm{i}<=(($ last-1)/iter)

set(gcf,'PaperType','usletter')

print('-dtiff','-r600',['13Cap_Upsilon_AR',num2str(10*AR)]);

end

hold off

figure $(14+17 * \mathrm{j})$

title(['AR ',num2str(AR)])

hold all

surf(squeeze(C_PSI(i,:,:)),squeeze(S_hat(i,:::)))

xlabel('\eta' ) 


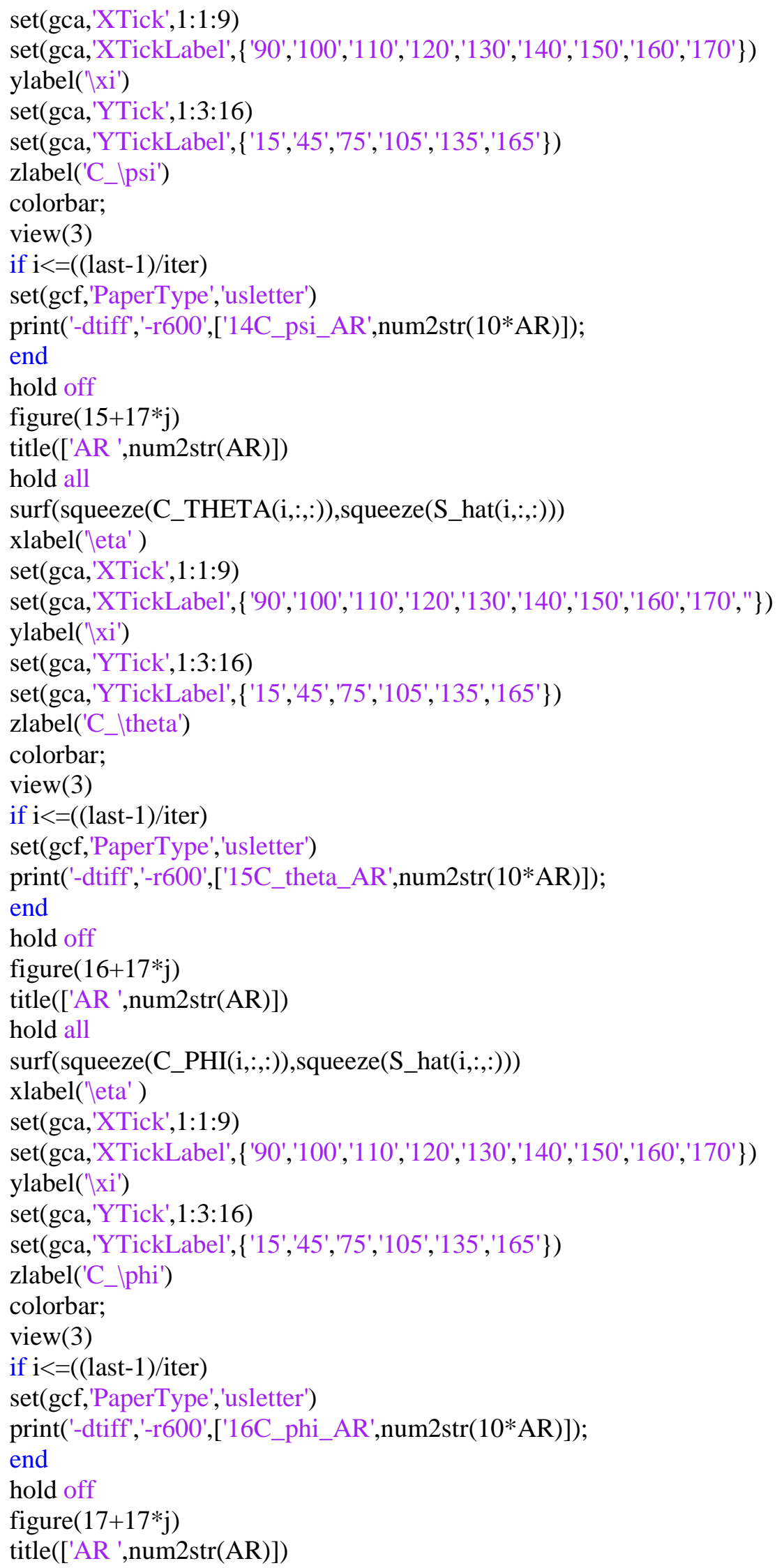




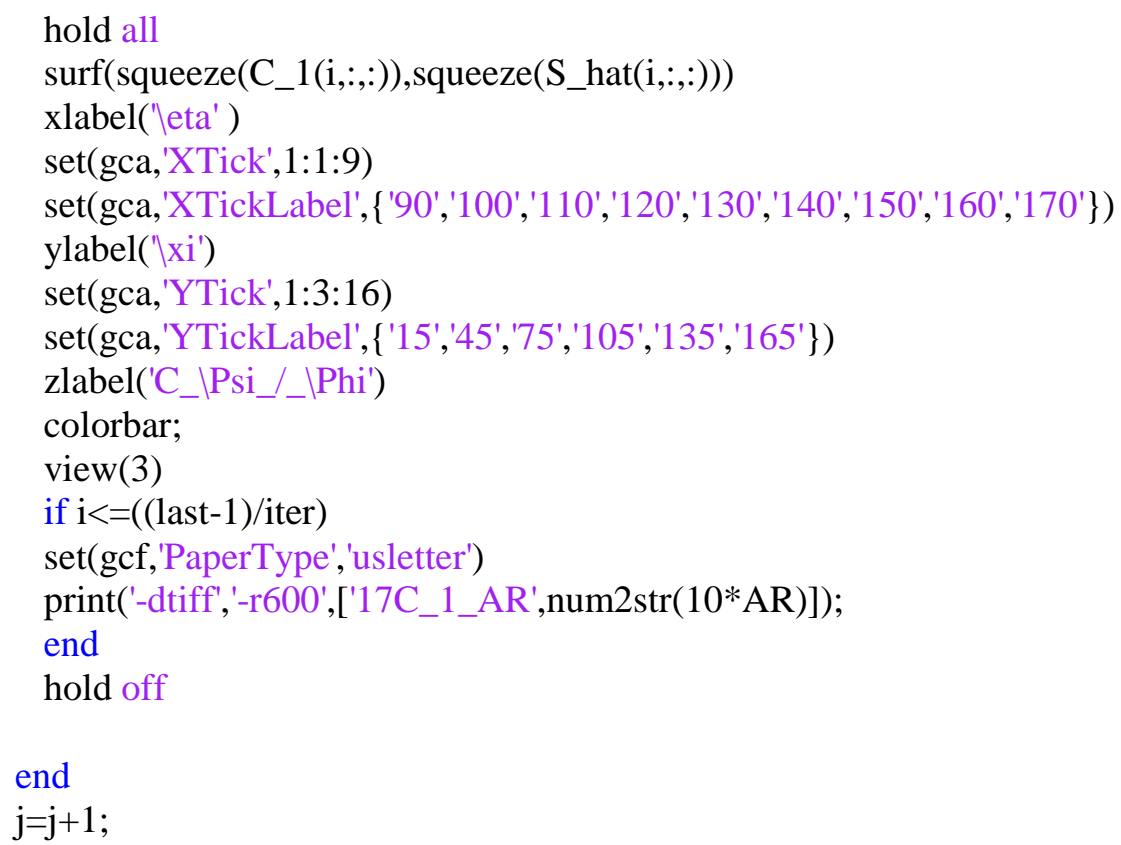

This is the Matlab code for numerical integration of dimensional equations.

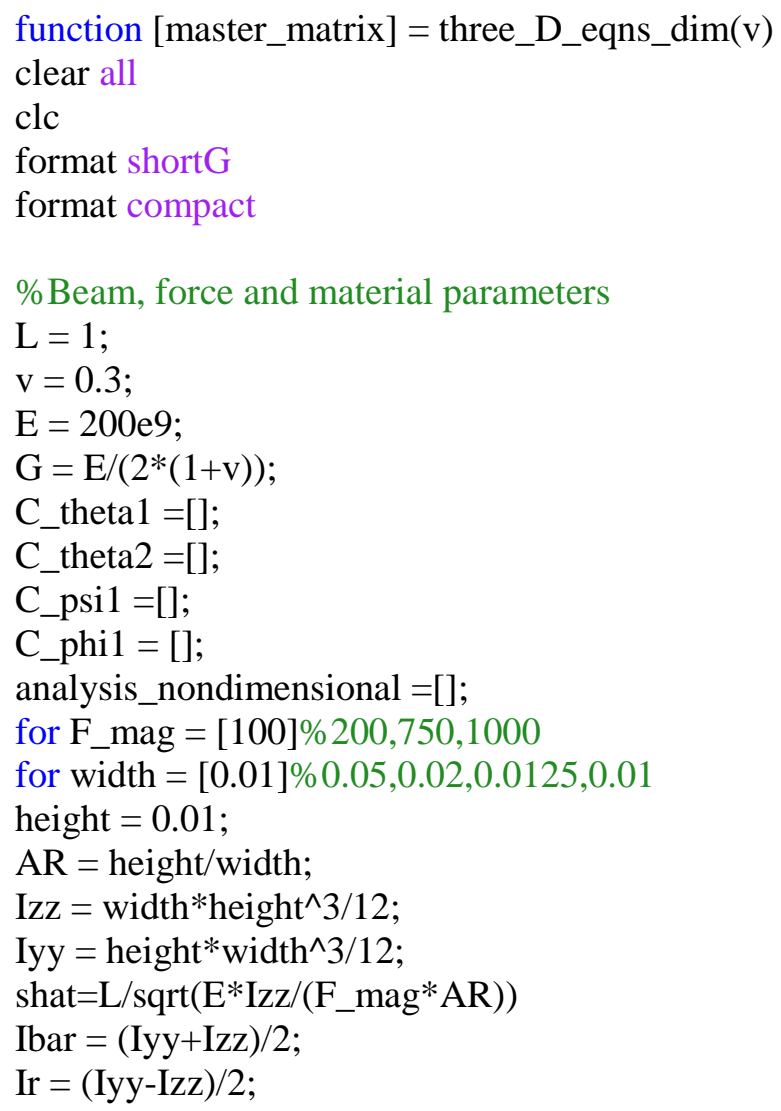




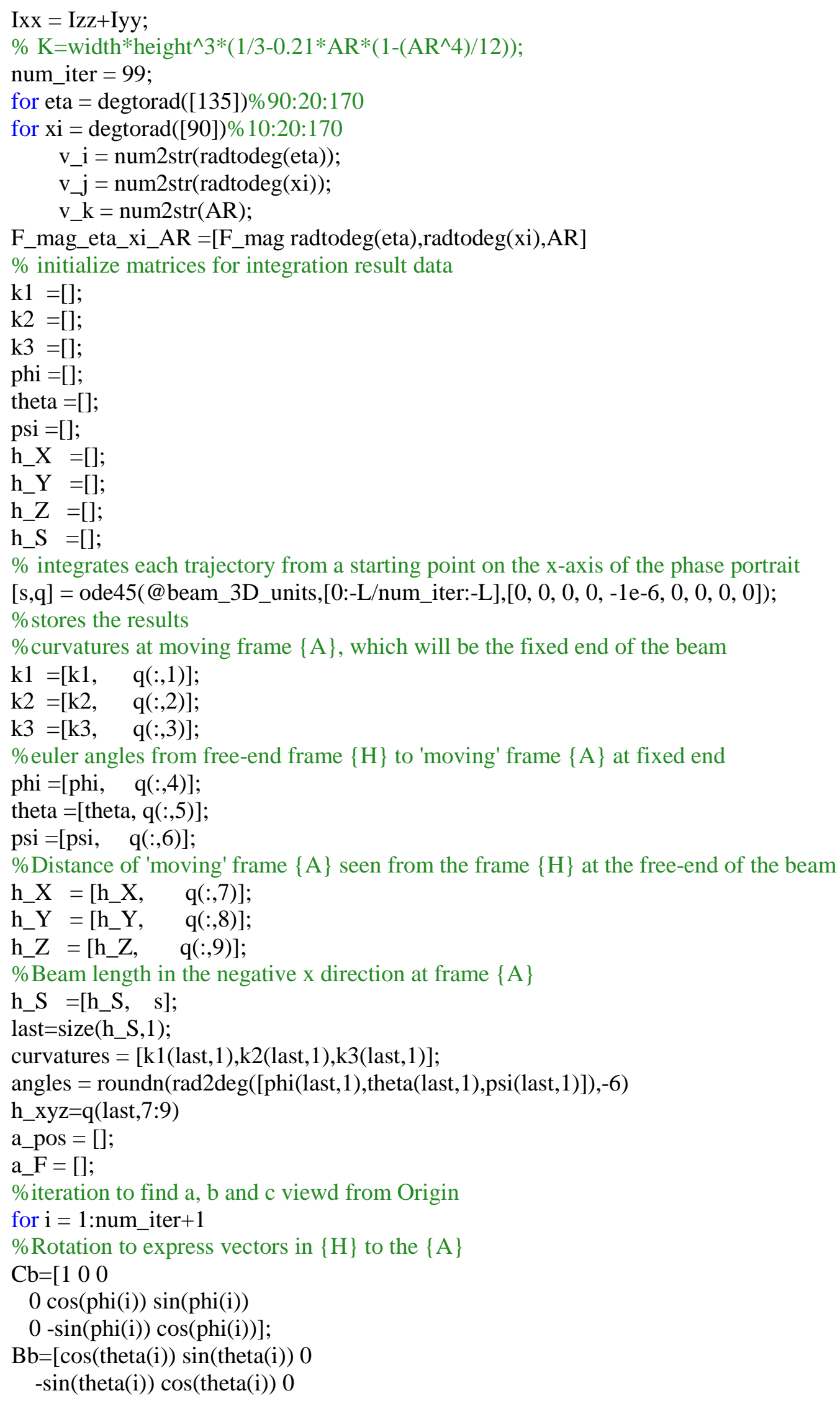


$\left.\begin{array}{lll}0 & 0 & 1\end{array}\right]$;

$\mathrm{Ab}=\left[\begin{array}{llll}1 & 0 & 0\end{array}\right.$

$0 \cos (\mathrm{psi}(\mathrm{i})) \sin (\mathrm{psi}(\mathrm{i}))$

0 -sin(psi(i)) cos(psi(i))];

a_R_h = Cb*Bb*Ab;

a_pos $=$ [a_pos;[-a_R_h*[h_X(i,1);h_Y(i,1);h_Z(i,1)]]'];

a_F $=$ [a_R_h*[F_mag*sin(eta)*cos(xi); F_mag*sin(eta)*sin(xi); F_mag*cos(eta)]]';

$\%$ th=atan2d(a_F(1,2),a_F(1,3))

end

a_R_h

$\%$ vector position of free end of the beam seen from frame $\{\mathrm{A}\}$

$\mathrm{abc}=[$ a_pos(size $($ a_pos, 1$), \mathrm{:})]$;

$\%(a, b, c)$ position coordinates viewed from $\{\mathrm{A}\}$ (last iteration)

$\mathrm{a}=\operatorname{abc}(:, 1)$

$\mathrm{b}=\mathrm{abc}(:, 2)$

$\mathrm{c}=\mathrm{abc}(:, 3)$

figure (1)

hold all

grid on

axis off

plot3(a_pos(:,1),-a_pos(:,3),a_pos(:,2),'LineWidth',4,'Color','k')

$\%$ \%fixed frame

\% line([0 0.1],[0 0],[0 0],'Color','r','LineWidth',2)

\% line([0 0],[0 -0.05],[0 0],'Color','g','LineWidth',2)

$\%$ line([0 0],[0 0],[0 .1],'Color','b','LineWidth',2)

\%component $\mathrm{x}$

line([0 a],[0 0],[0 0],'Color','k','LineWidth',1,'LineStyle','-.')

$\%$ component y

line([a a],[0 b],[0 0],'Color','k','LineWidth',1,'LineStyle','-.')

\%component z

line([a a],[b b],[0 -c],'Color','k','LineWidth',1,'LineStyle','-.')

$\%$

\% quiver3(a,-b,c,a_R_h(1,1),-a_R_h(2,1),a_R_h(3,1),0.1,'Color','r')

\% quiver3(a,-b,c,a_R_h(1,2),-a_R_h(2,2),a_R_h(3,2),0.1,'Color','g')

\% quiver3(a,-b,c,a_R_h(1,3),-a_R_h(2,3),a_R_h(3,3),0.1,'Color,''b')

view([30 45])

set(gcf,'Color',[1,1,1])

hold off

$\%$ Force components viewed from $\{\mathrm{A}\}$ (last iteration)

$\%$ Non-follower equivalent force

a_F $=$ [a_F(size $\left.\left.\left(a \_F, 1\right),:\right)\right]$;

$\%$ Non-follower angles

alpha $=$ roundn $\left(\operatorname{rad} 2 \operatorname{deg}\left(\operatorname{acos}\left(\mathrm{a} \_\mathrm{F}(1,3) / \mathrm{F} \_\mathrm{mag}\right)\right),-3\right)$

beta $=$ roundn $\left(\operatorname{rad} 2 \operatorname{deg}\left(\operatorname{atan} 2\left(\mathrm{a} \_\mathrm{F}(1,2), \mathrm{a} \_\mathrm{F}(1,1)\right)\right),-3\right)$;

beta $=($ beta $<0) \cdot *(360 *$ ones $(\operatorname{size}($ beta, 1$), 1)+$ beta $)+($ beta $>0) . *$ beta

Follower_F $=$ [F_mag*sin(eta)*cos(xi), F_mag*sin(eta)*sin(xi), F_mag*cos(eta)];

Non_Follower_F $=$ [F_mag*sind(alpha)*cosd(beta); F_mag*sind(alpha)*sind(beta);

F_mag*cosd(alpha)]';

$\%$ Cap_phi angle rotation about the $\mathrm{x}$-axis in the $\{\mathrm{A}\}$ frame

Cap_phi = atan2(c,b);

Cap_phi_d=rad2deg(Cap_phi) 


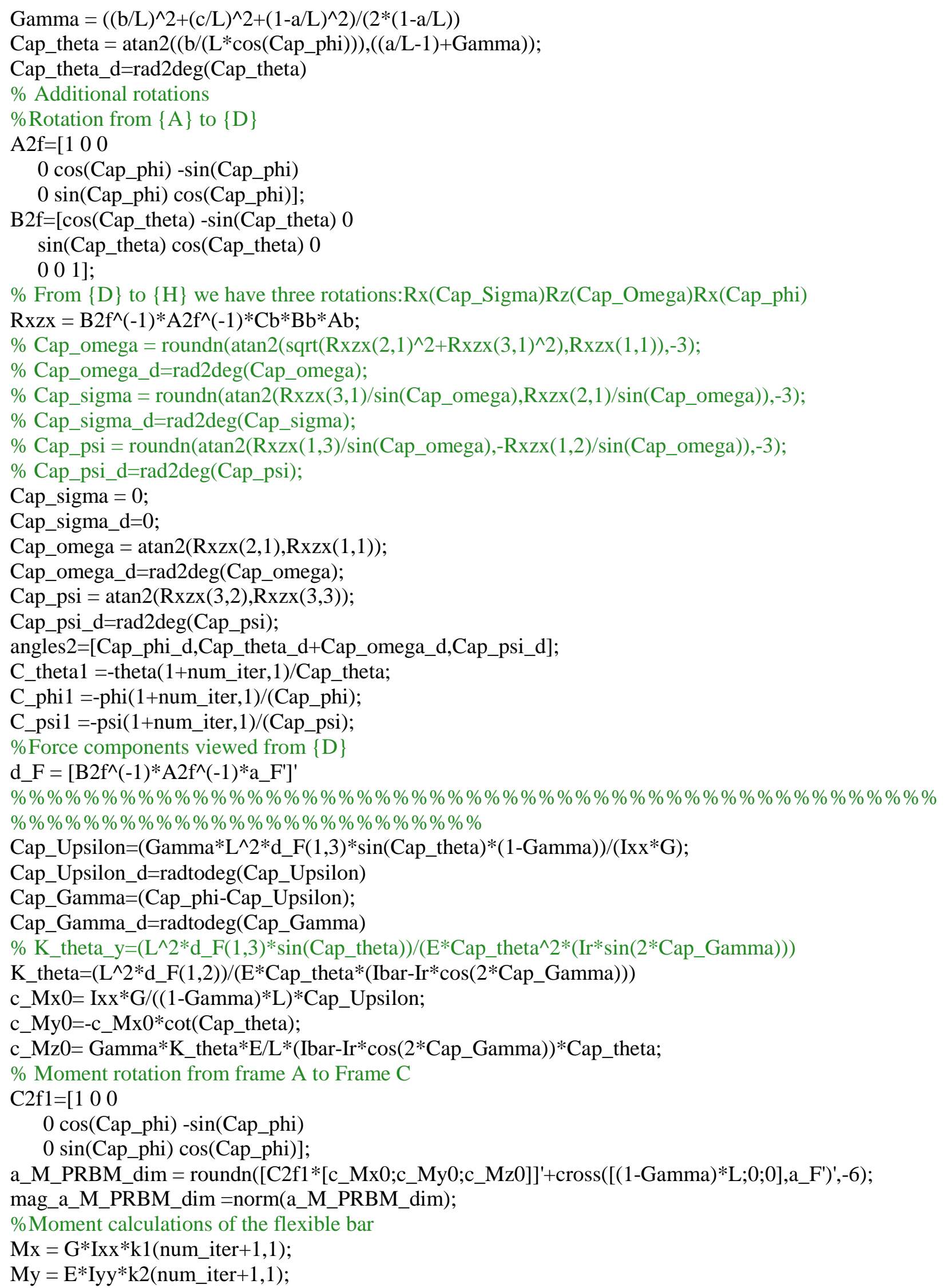




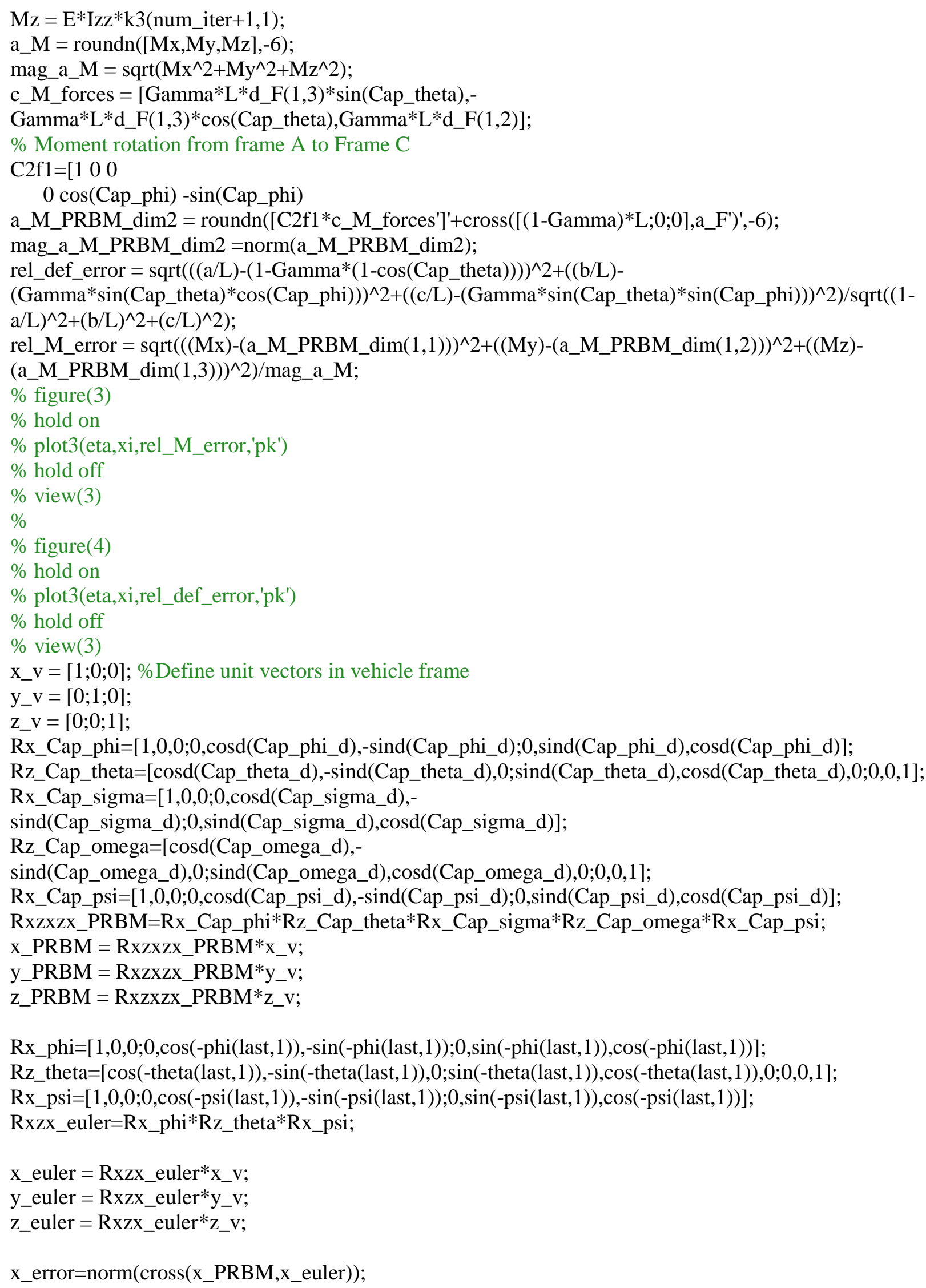




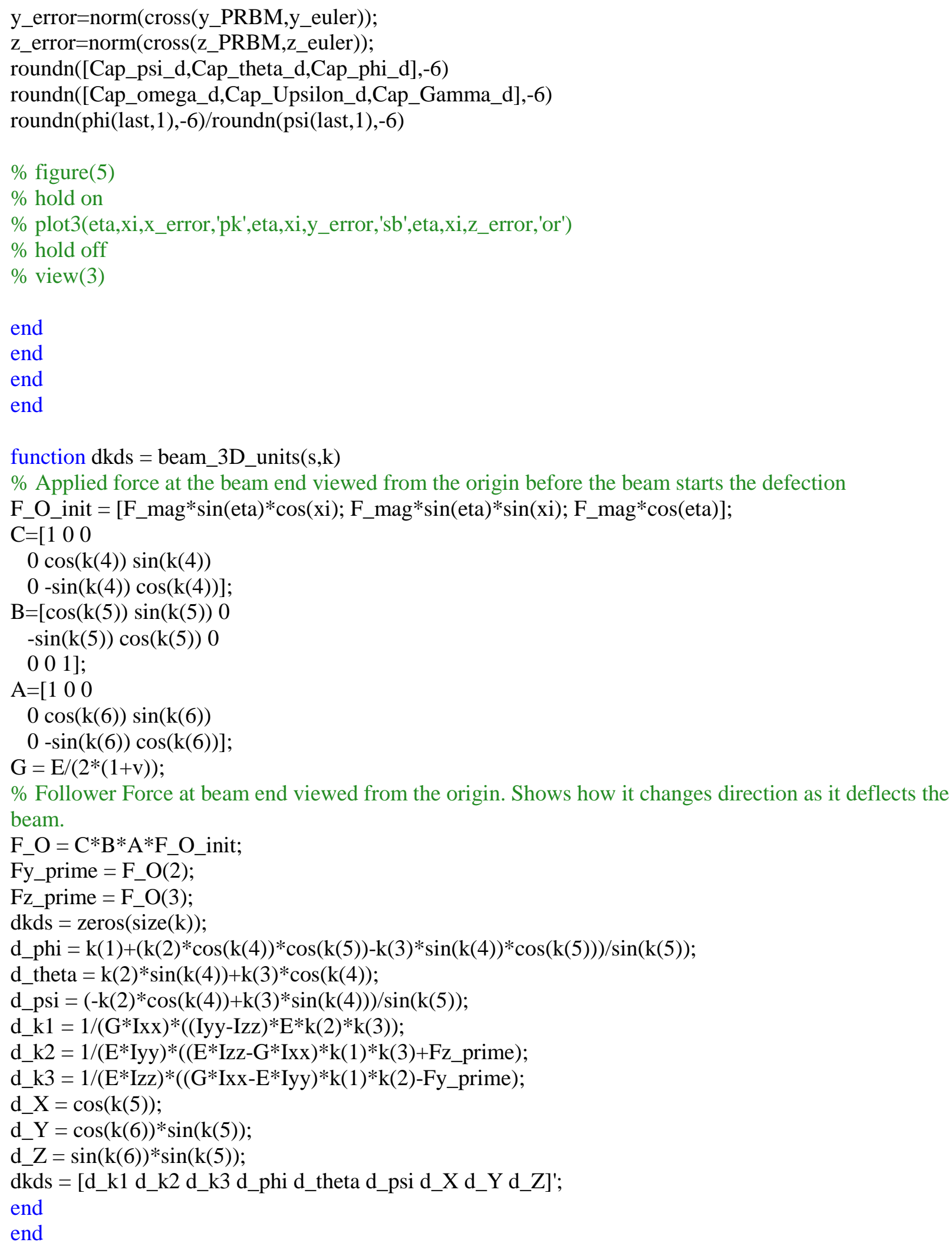


This is the ANSYS code for the spatial beam verification.

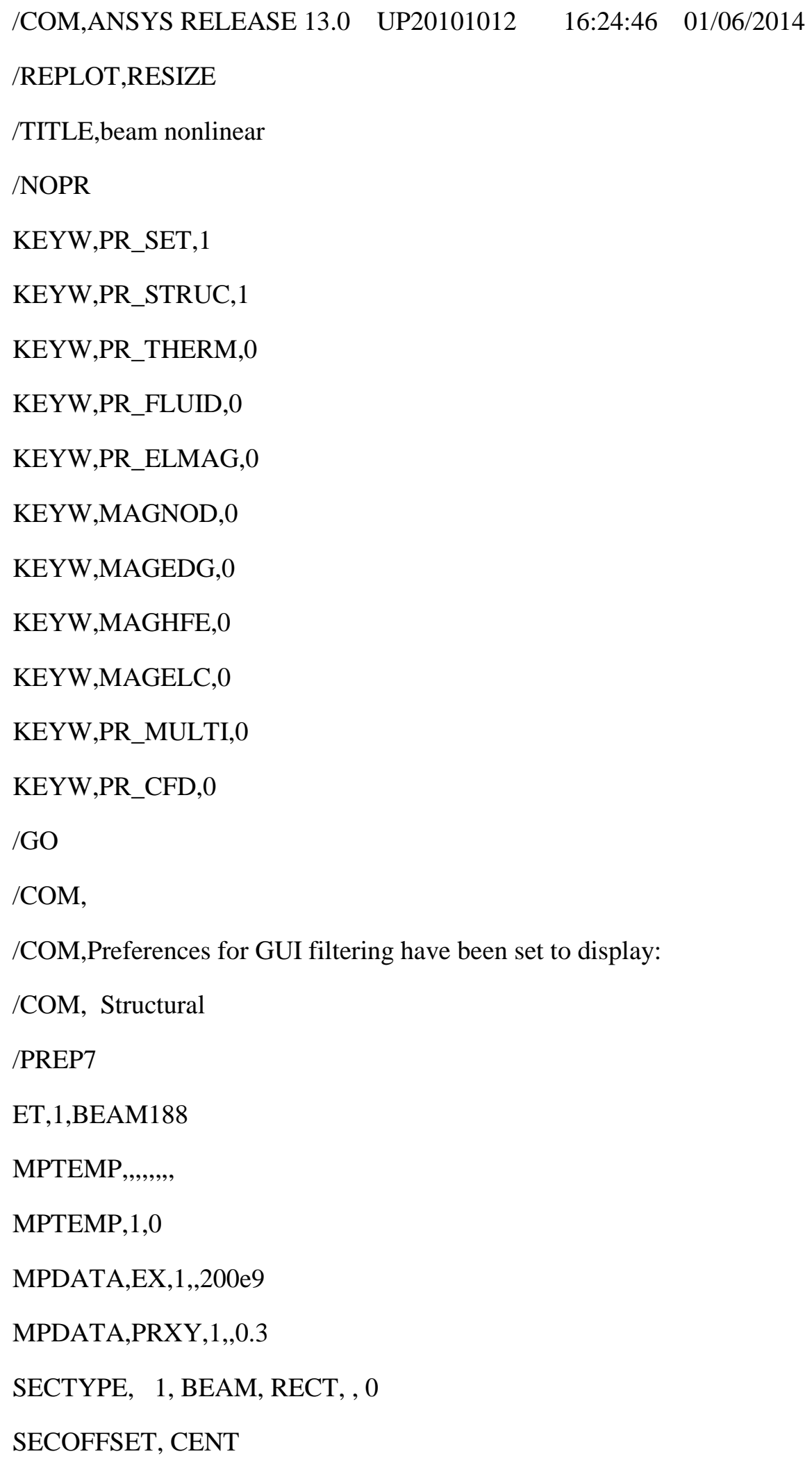


SECDATA,0.01,0.02,0,0,0,0,0,0,0,0,0,0

$\mathrm{K}$, ,,,

$\mathrm{K}, 2,1$,,

LSTR, 1, 2

LESIZE,ALL,0.01, , , ,1, , ,1,

LMESH, 1

FINISH

/SOL

ANTYPE,0

NLGEOM,1

FLST,2,1,3,ORDE,1

FITEM,2,1

/GO

DK,P51X, , , ,0,ALL, , , , , ,

FLST,2,1,3,ORDE,1

FITEM,2,2

/GO

FK,P51X,FX,-268.97

FLST,2,1,3,ORDE,1

FITEM,2,2

/GO

FK,P51X,FY,561.08

FLST,2,1,3,ORDE,1

FITEM,2,2

/GO

FK,P51X,FZ,-418.74

/STATUS,SOLU

SOLVE 